University of Louisville

ThinkIR: The University of Louisville's Institutional Repository

$12-2020$

\title{
The effects of whole life, low dose cadmium exposure on high fat diet-induced nonalcoholic fatty liver disease and the role of zinc.
}

Jamie Lynn Young

University of Louisville

Follow this and additional works at: https://ir.library.louisville.edu/etd

\section{Recommended Citation}

Young, Jamie Lynn, "The effects of whole life, low dose cadmium exposure on high fat diet-induced nonalcoholic fatty liver disease and the role of zinc." (2020). Electronic Theses and Dissertations. Paper 3561.

https://doi.org/10.18297/etd/3561

This Doctoral Dissertation is brought to you for free and open access by ThinkIR: The University of Louisville's Institutional Repository. It has been accepted for inclusion in Electronic Theses and Dissertations by an authorized administrator of ThinkIR: The University of Louisville's Institutional Repository. This title appears here courtesy of the author, who has retained all other copyrights. For more information, please contact thinkir@louisville.edu. 
THE EFFECTS OF WHOLE LIFE, LOW DOSE CADMIUM EXPOSURE ON HIGH FAT DIET-INDUCED NONALCOHOLIC FATTY LIVER DISEASE AND THE ROLE OF ZINC

\author{
By \\ Jamie Lynn Young \\ B.A., University of Maine at Farmington, 2007 \\ M.S., University of Louisville, 2018 \\ A Dissertation \\ Submitted to the Faculty of the \\ School of Medicine of the University of Louisville \\ In Partial Fulfillment of the Requirements \\ for the Degree of \\ Doctor of Philosophy in Pharmacology and Toxicology \\ Department of Pharmacology and Toxicology \\ University of Louisville \\ Louisville, Kentucky
}

December 2020 
Copyright 2020 by Jamie Lynn Young

All rights reserved 

THE EFFECTS OF WHOLE LIFE, LOW DOSE CADMIUM EXPOSURE ON HIGH FAT DIET-INDUCED NONALCOHOLIC FATTY LIVER DISEASE AND THE ROLE OF ZINC

\author{
By \\ Jamie Lynn Young \\ B.A., University of Maine at Farmington, 2007 \\ M.S., University of Louisville, 2018
}

Dissertation Approved on

$11 / 12 / 2020$

by the following Dissertation Committee:

Lu Cai, Ph.D.

J. Christopher States, Ph.D.

Michael L. Merchant, Ph.D.

Maiying Kong, Ph.D.

ShaoYu Chen, Ph.D. 


\section{DEDCIATION}

This dissertation is in loving dedication to John P. Wise Sr.

for his constant support,

patience,

guidance,

and unquestionable belief in me, always. 


\section{ACKNOWLEDGEMENTS}

First, I would like to thank my mentor, Dr. Lu Cai for his guidance and support in my research and also for his confidence in my abilities as a scientific researcher. His support has been instrumental in the success of my research and my growth as a person and scientist. I would also like to thank my other committee members, Dr. Chris States, Dr. Michael Merchant, Dr. Maiying Kong and Dr. ShaoYu Chen for their comments, assistance, and support throughout this endeavor.

I have been fortunate to have amazing support from my peers. I would like to thank past and present members of the Cai Laboratory, the Tan Laboratory and the Wise Laboratory as they have all supported me throughout my graduate school career, whether at the bench, in the animal facility, at a conferences or in the classroom. From the Cai lab: Wenqian Zhou, Feng Li, Bin Zhou, Hongbo Men, Jiqun Wang, Tingwen Ge, Li Zhou, and Dr. Jonny Wise Jr. From the Tan lab: Dr. Yi Tan, Qian Lin (Zoe), Junhong He and Chengkui Yang. From the Wise lab: Dr. John Wise Sr., Dr. Sandra Wise, Dr. Rachel Speer, Jenifer Toyoda, Taylor CroomPerez, Haiyan Lu, Idoia Meaza and Aggie Williams. I would like to thank Dr. Jason $\mathrm{Xu}$ for his work running the ICP-MS as well as for always being willing to help out at the last minute. I would also like to thank Dr. Maiying Kong and Qian Xu for their help with the biostatistical analysis of the body weight data. 
Next, I would like to thank the Integrated Programs in Biomedical Sciences (IPIBS) and the Department of Pharmacology and Toxicology for providing the first two years of my funding as well as opportunities to ensure my success. I would also like to thank Dr. David Hein, the Department Chair for his guidance and continued support and for providing myself and other students at the University of Louisville the opportunity to become a National Institute of Environmental Health Sciences T32 Trainee. I would also like to thank Dr. Leah Siskind for helping me navigating my way through the graduate program.

In addition to the family and friend mentioned above I would also like to thank Dr. Catherine Wise and Dr. James Wise for always being willing to proof read manuscripts, give insight into experimental design and help me network. Finally I must thank my daughters, Emma and Addilyne, for simply being awesome and thinking it's cool that mommy is going to be a "doctor scientist". 


\begin{abstract}
THE EFFECTS OF WHOLE LIFE, LOW DOSE CADMIUM EXPOSURE ON HIGH FAT DIET-INDUCED NONALCOHOLIC FATTY LIVER DISEASE AND THE ROLE OF ZINC
\end{abstract}

Jamie L. Young

November 12, 2020

Nonalcoholic fatty liver disease (NAFLD) is a major global public health concern affecting more than $25 \%$ of the world's population. Although obesity is a major risk factor for NAFLD, it cannot account for many cases, indicating the importance of other factors such as sex and environmental exposures. Cadmium exposure is implicated in the development of NAFLD; however, the influence of early-life, in utero cadmium exposure on the development of diet-induced NAFLD is poorly understood. Therefore, we developed an in vivo, multiple-hit model to study the effect of whole-life, low-dose cadmium exposure on high fat diet (HFD)-induced NAFLD. Additionally, we investigated the impact of dietary zinc supplementation on disease outcome as both obesity and cadmium disrupt zinc homeostasis and zinc deficiency is common in NAFLD patients. Adult male and female C57BL/6J mice fed normal diets (ND) were exposed to $0,0.5$ or $5 \mathrm{ppm}$ cadmium-containing drinking water for 14 weeks before breeding. At weaning, offspring were fed ND or HFD and continued on the same drinking water regimen as their parents. Male 
offspring were further subdivided into diets containing 30 or $90 \mathrm{mg} \mathrm{zinc/4057} \mathrm{kcal,}$ representing normal and zinc supplemented diet, respectively. Cadmium exposure altered HFD-associated weight gain and insulin resistance in males, but not females. In males only, cadmium exposure altered HFD-induced liver injury and NAFLD. Specifically, $0.5 \mathrm{ppm}$ cadmium exposure rescued the adverse health effects of HFD while 5 ppm cadmium exposure exacerbated outcomes. Further, HFD blunted the response of metallothionein in mice exposed to $5 \mathrm{ppm}$ cadmium but enhanced the response in male mice exposed to $0.5 \mathrm{ppm}$ cadmium, suggesting a possible mechanism for cadmium altering HFD-induced NAFLD. Zinc supplementation rescued the adverse effects cause by HFD and 5 ppm cadmium exposure. Interestingly, in 5 ppm cadmium-exposed, HFD-fed female mice hepatic zinc levels were similar to levels in males after zinc supplementation. Overall, results from this study confirm the multi-hit nature of NAFLD, show whole life, low dose cadmium exposure alters HFD-induced NAFLD, and suggest a potential therapeutic role for zinc. Furthermore, this study highlights the importance of sex as a risk factor in disease development. 


\section{TABLE OF CONTENTS}

\section{PAGE}

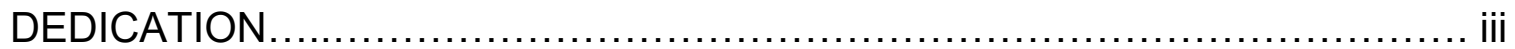

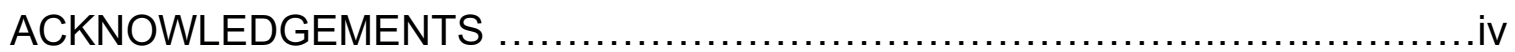

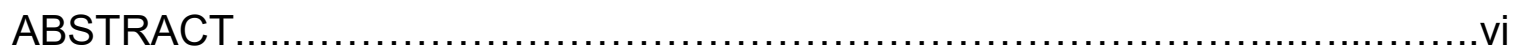

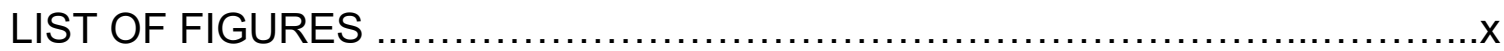

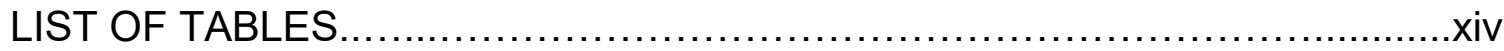

CHAPTER 1: INTRODUCTION ................................................

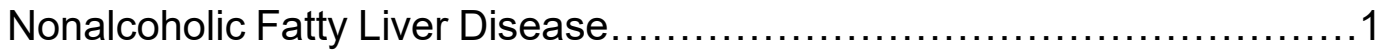

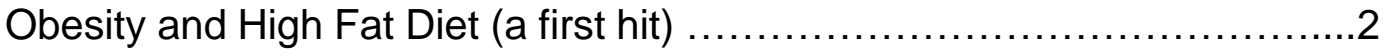

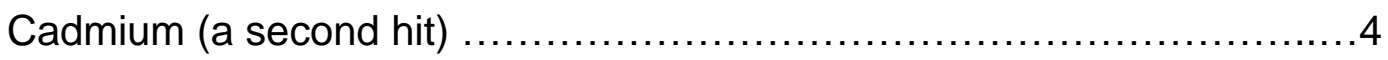

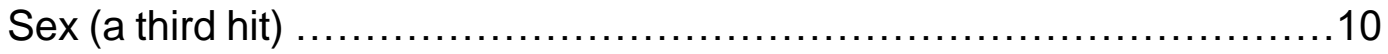

Developmental Origins of Health and Disease (an important factor) ......13

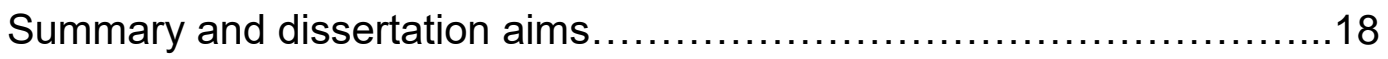

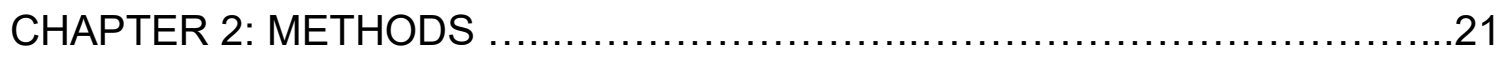

Animal models and exposures.........................................

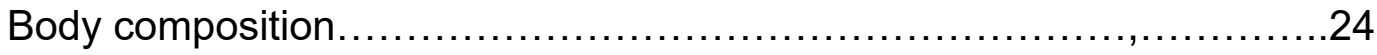

Glucose tolerance test...............................................25

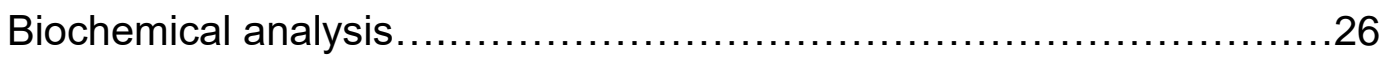

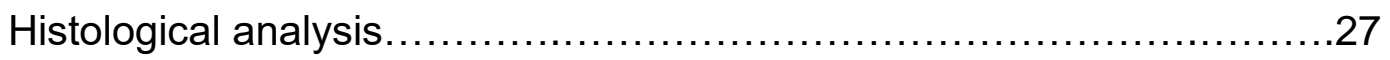

Total RNA isolation, cDNA synthesis and qRT-PCR ....................28 
Protein extraction for western blot.

Western blot analysis.............................................

Western blot analysis for metallothionein...........................32

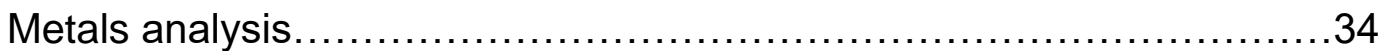

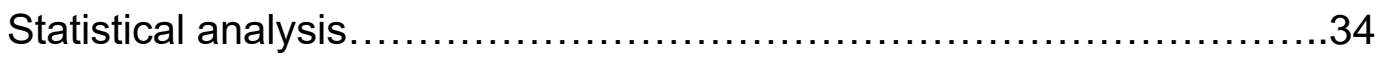

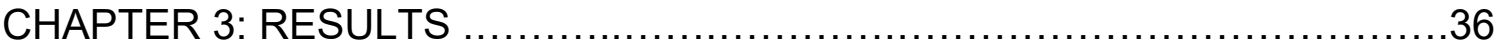

Aim 1: Measure the impact of whole-life, chronic cadmium exposure on HFD-

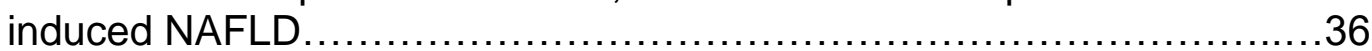

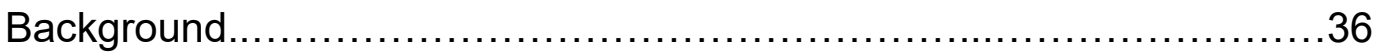

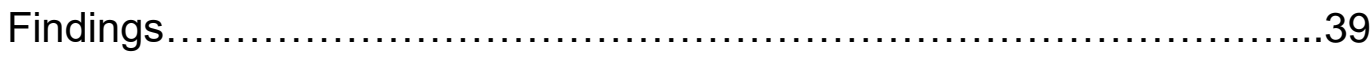

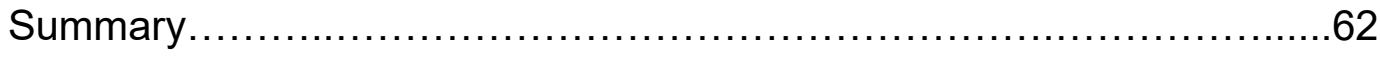

Aim 2. Show zinc supplementation protects against cadmium-enhanced, HFD-

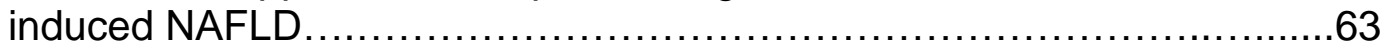

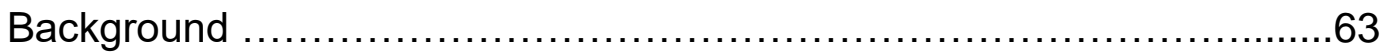

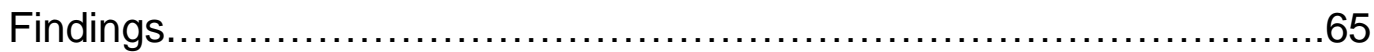

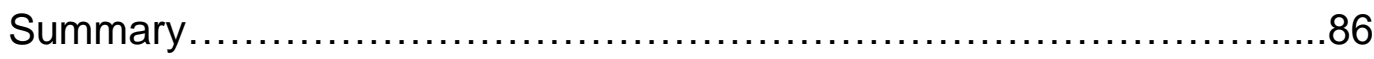

Aim 3. Determine the role of sex in cadmium-enhanced, HFD-induced NAFLD.87

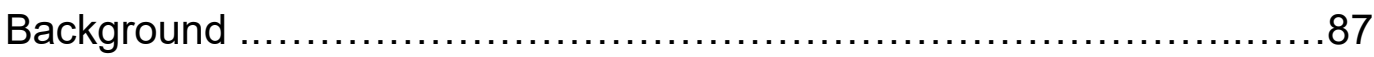

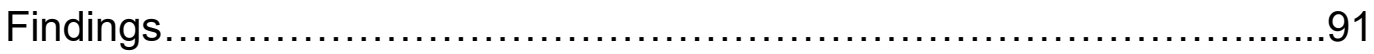

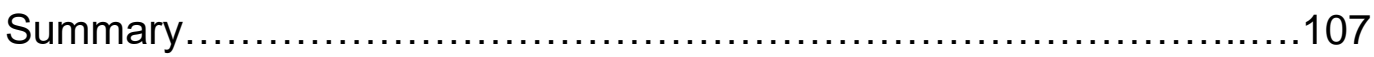

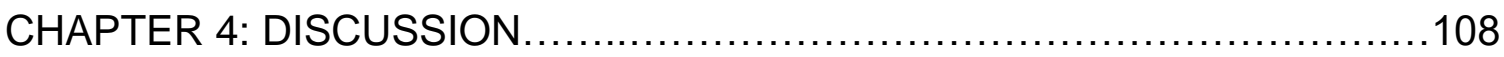

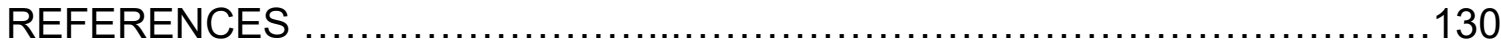

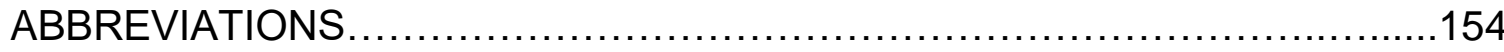

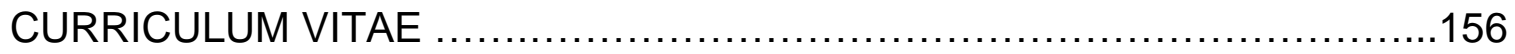




\section{LIST OF FIGURES}

FIGURE

PAGE

1.1. 'Multiple hit' hypothesis of NAFLD progression ...............................

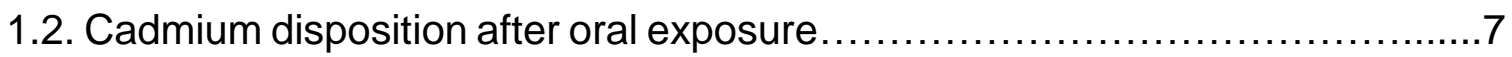

1.3. Summary of the effects of placental cadmium accumulation and the nonlethal adverse birth outcomes that may lead to increased susceptibility of disease

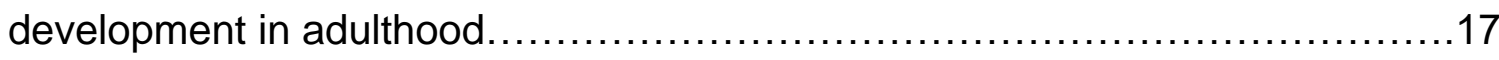

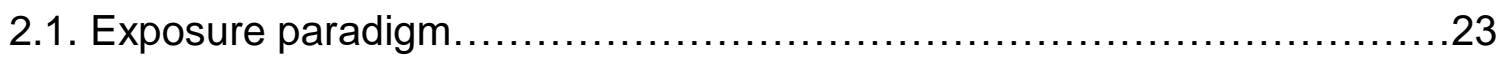

3.1. Model of whole life exposure of male mice to cadmium and high fat diet....38

3.2. Effects of high fat diet on body weight and composition in male mice.........41

3.3. Effects of high fat diet on glucose handling and plasma insulin in male

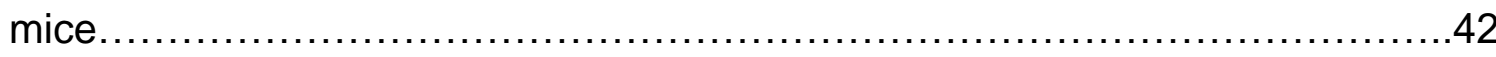

3.4. Effects of high fat diet on liver injury and NAFLD in male mice...............43 3.5. Hepatic cadmium levels and weekly administered cadmium intake in male

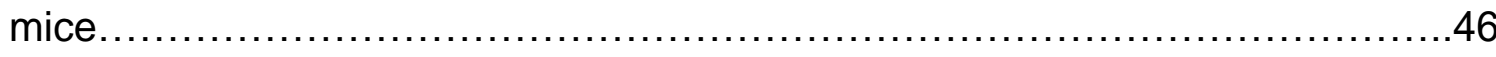

3.6. Effects of whole life cadmium exposure and high fat diet on metallothionein

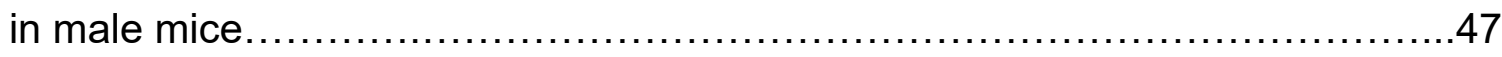

3.7. Effects of whole life cadmium exposure and high fat diet on body weight and

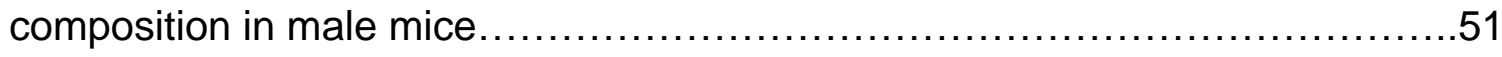


3.8. Effects of whole life exposure to cadmium and high fat diet on glucose handling and plasma insulin in male mice.

3.9. Effects of whole life cadmium exposure and high fat diet on liver injury in male mice .56

3.10. Effects of whole life cadmium exposure and high fat diet on NAFLD in male mice.

3.11. Effects of whole life cadmium exposure and high fat diet on biochemical measures of NAFLD in male mice

3.12. Effects of whole life cadmium exposure and high fat diet on SREBP-1 mediated lipogenesis.

3.13. Model of whole life exposure of male mice to cadmium and high fat diet supplemented with zinc. .66

3.14. Effects of zinc supplementation and high fat diet on body weight and composition in male mice.

3.15. Effects of zinc supplementation and high fat diet on glucose handling and plasma insulin in male mice. .69

3.16. Effects of zinc supplementation and high fat diet on liver injury in male mice.

3.17. Effects of zinc supplementation and high fat diet on NAFLD in male mice.

3.18. Hepatic cadmium and zinc levels and weekly administered cadmium intake in zinc supplemented male mice. 
3.19. Effects of zinc supplementation, cadmium exposure and high fat diet on metallothionein in male mice.

76

3.20. Effects of zinc supplementation on cadmium-exacerbated, high fat dietinduced weight gain and body composition in male mice.

78

3.21. Effects of zinc supplementation on cadmium and high fat diet-altered

glucose handling and plasma insulin in male mice.

3.22. Zinc supplementation recues cadmium-exacerbated, high fat diet-induced

liver injury in male mice

3.23. Zinc supplementation recues cadmium-exacerbated, high fat diet-induced

NAFLD in male mice.

3.24. Zinc supplementation recues cadmium-exacerbated, high fat diet-induce hepatic triglycerides in male mice.

3.25. Model of whole life exposure of female mice to cadmium and high fat diet. 90

3.26. Effects of high fat diet on body weight and composition in female mice....92 3.27. Effects of high fat diet on glucose handling and plasma insulin in female mice. .93

3.28. Effects of high fat diet on liver injury and NAFLD in female mice. .94

3.29. Hepatic cadmium levels and weekly administered cadmium intake in female mice. 96

3.30. Effects of whole life cadmium exposure and high fat diet on body weight and composition in female mice. 
3.31. Effects of whole life exposure to cadmium and high fat diet on glucose handling and plasma insulin in female mice................................100 3.32. Effects of whole life cadmium exposure and high fat diet on liver injury in

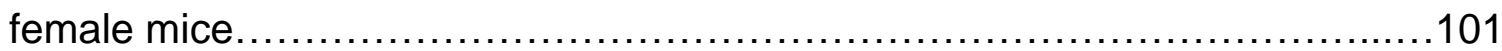
Figure 3.33. Effects of whole life cadmium exposure and high fat diet on NAFLD in female mice. 102 3.34. Effects of whole life cadmium exposure and high fat diet on biochemical measures of NAFLD in female mice. 104 3.35. Hepatic zinc levels in female mice 106 


\section{LIST OF TABLES}

TABLE

PAGE

3.1. Summary of the effects of zinc supplementation in control mice ..............86

3.2. Summary of zinc supplementation effects in cadmium exposed, high fat diet

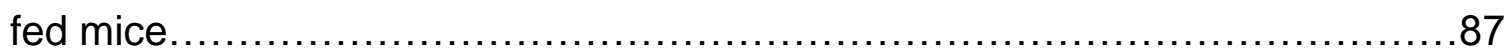




\section{CHAPTER 1: INTRODUCTION}

\section{Nonalcoholic Fatty Liver Disease}

Nonalcoholic fatty liver disease (NAFLD) is the most common cause of chronic liver disease with a global prevalence of between 25-45\% (Rinella, 2015; Younossi et al., 2018). In the United States, an estimated 35,000 people die annually as a result of NAFLD-related complications (Paik et al., 2019). Unfortunately, the prevalence of NAFLD has almost doubled over the past twenty years and remains on the rise (Welsh et al., 2013); increasing in parallel with two well-characterized risk factors of chronic liver disease: obesity and diabetes (Williams et al., 2011; Chalasani et al., 2012). In fact, between 75 and 100 million people in the U.S. are likely to have NAFLD, based on the estimation that $68 \%$ of the adult population in U.S. is either overweight or obese (Rinella, 2015).

First described in 1980, NAFLD presents with a wide clinical spectrum of outcomes involving excessive fat accumulation in the liver (fat deposits in $>5 \%$ of hepatocytes) not due to alcohol consumption (Rinella, 2015). Histologically, NAFLD is divided into two categories: nonalcoholic fatty liver (NAFL) and nonalcoholic steatohepatitis (NASH). Patients in the first category (NAFL) present with simple steatosis while patients in the second category (NASH) present with cellular damage and inflammation (Ludwig et al., 1980; Kleiner et al., 2005; Rinella, 2015). The spectrum of disease outcomes range from NAFL to NASH to cirrhosis, 
and ultimately progression to hepatocellular carcinoma (Byrne and Targher, 2015; Asrih and Jornayvaz, 2015). Approximately, $25 \%$ of subjects will progress from simple steatosis on to more severe, chronic liver injury (Rinella, 2015; Buzzetti et al., 2016), highlighting that the progression of NAFLD is complex and multifactorial. Initially, to explain disease progression, a 'two hit' hypothesis was formulated by Day and James (1998) suggesting a factor such as obesity serves as a 'first hit' which sensitizes the liver to further insult, with a 'second hit' causing disease progression from NAFL to NASH. However, over the past 20 years it has become clear that the numerous molecular and metabolic changes that take place throughout NAFLD progression are inadequately explained by the 'two hit' hypothesis, making way for the current 'multiple hit' hypothesis (Buzzetti et al., 2016). As such, disease progression is determined by multiple parallel factors including diet, genetics, sex and environmental factors (Figure 1.1).

\section{Obesity and High Fat Diet (a first hit)}

Obesity is a major public health concern affecting over 312 million people worldwide. By the year 2050, the number of obese individuals in the United States alone is expected to double (Fakhouri et al., 2013). A chronic imbalance of calorie intake and calorie expenditure result in larger and more numerous fat cells in the body, and subsequently obesity (Smilowitz et al., 2010). One of the major variables contributing to obesity is consumption of high fat diets (Golay and Bobbioni, 1997),

which have become easily accessible and served in large portions and contribute to today's "obesogenic environment" (Poston and Foreyt, 1999). 


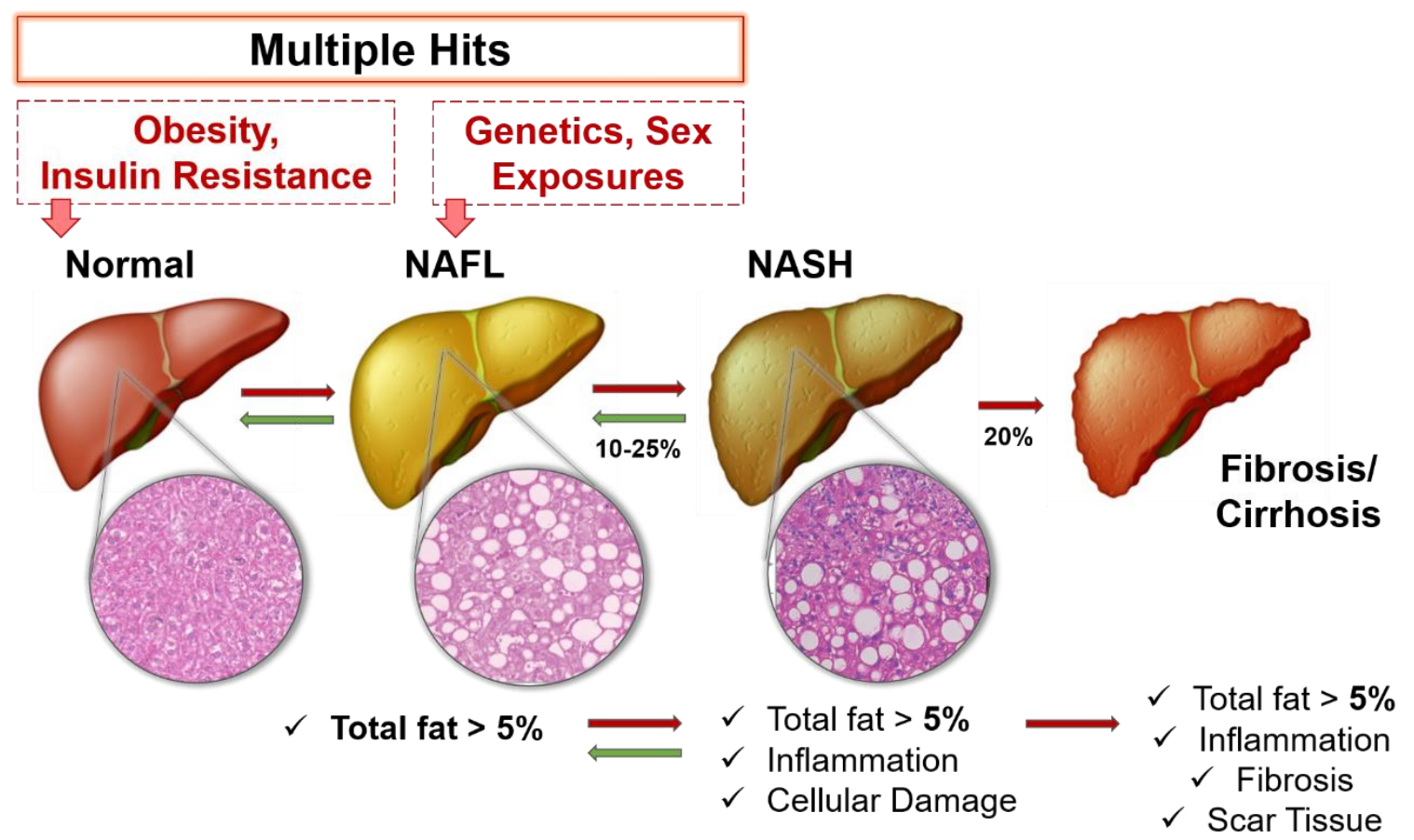

Figure 1.1 'Multiple hit' hypothesis of NAFLD progression. NAFLD is a spectrum of diseases ranging from simple lipid accumulation to cirrhosis and ultimately hepatocellular carcinoma. NAFLD progression is complex and multifactorial. The 'multiple hit' hypothesis suggest disease progression is determined by multiple parallel factors including obesity, insulin resistance, genetics, sex and environmental exposures. Red arrows indicate disease progression. Green arrows indicate disease reversibility. 
In humans, high fat diets easily induce obesity (Hariri and Thibault, 2010). In fact, epidemiological studies in the United States, Canada and China have all shown the incidence of obesity increases in parallel with increases in dietary fat consumption (George et al., 1990; Popkin et al., 1993; Saris et al., 2000; Tucker et al., 1992). Since the early 1950's, when Fenton and Dowling (1953) created the first dietary obesity model using a high fat diet in rats, animal models have been widely utilized for experiments on dietary obesity (Hariri and Thibault, 2010). Animal models of dietary obesity have allowed for the advancement of our understating of several health conditions in which obesity is a major risk factor including type 2 diabetes, hypertension and NAFLD (Ogden et al., 2012).

Although obesity is the primary risk factor for the development of NAFLD, approximately $20 \%$ of normal weight people develop the disease, indicating other risk factors are involved (Younossi et al., 2018). One such risk factor is exposure to the non-essential metal cadmium, consistent with the fact the liver is a major target organ of cadmium toxicity and accumulation (Arroyo et al., 2012).

\section{Cadmium (a second hit)}

Cadmium is a naturally occurring heavy metal with no known biological function in humans and has been recognized as an occupational and environmental risk factor for decades (ATSDR, 2012; Tinkov et al., 2017). Ranking number 7 on the Agency for Toxic Substances and Disease Registry list of environmental chemical hazards (ATSDR, 2012), cadmium is one of the most common and detrimental metals present in our environment (Jacobo-Estrada et 
al., 2017). Approximately 25,000 tons of cadmium are naturally released into the environment annually (Fatima et al., 2019). For example, natural releases of cadmium into the air occur through volcanoes and forest fires while releases into rivers and water bodies occur through the weathering of rocks (Fatima et al., 2019). Additional releases of cadmium into the environment occur through human activity. Although cadmium was discovered in 1817 , it was not utilized commercially until the late $19^{\text {th }}$ century when it was used primarily in paint pigments (Llewellyn, 1994; IARC, 1993). Over the last century, exposure to cadmium has dramatically increased (IPCS, 1992) due to its use in the production of batteries, pigments and plastics. Anthropogenic sources of cadmium include mining, burning of fossil fuels and incineration of household wastes, all of which play a significant role in generating concentrated sources of cadmium and releasing it into the environment (Jarup and Akesson, 2009). According to the Environmental Protection Agency (EPA) cadmium has been found in 1,014 of the 1,669 current or former sites on the National Priorities List (NPL) of hazardous waste sites. Although these sites are destined for long-term clean-up, they pose a dangerous source of cadmium exposure (ATSDR, 2012).

Cadmium released into the soil and water can accumulate in plants and organisms that subsequently enter the food supply. Therefore, ingestion of contaminated food and water are the primary source of cadmium exposure in the non-smoking, general population with an average daily intake of between 4 and 26 $\mu \mathrm{g} /$ day (Choudhury et al., 2001; Martorell et al., 2011; Kim et al., 2018). One of the most dangerous characteristics of cadmium is that it is a cumulative toxicant in 
which the body burden increases with age. The biological half-life of cadmium is between 4 and 37 years, depending on the organ (ATSDR, 2012). The kidney and the liver are the primary target tissues for cadmium accumulation accounting for approximately $50 \%$ of the body's cadmium burden (HSDB, 2006).

Cadmium has similar physical and chemical properties to essential divalent metals such as zinc, iron and calcium. Therefore, through the process of ionic and molecular mimicry, cadmium enters cells without the need of its own specific transport proteins (Arroyo et al., 2012; Vesey, 2010). For example, studies have shown cadmium ions use the divalent metal transporter 1 (DMT1, protein encoded by the gene SLC11A2) as well as the metal transporter 1 (MTP1, protein encoded by the gene SLC40A1) for cellular transport, both found on the membrane of the enterocytes lining the intestinal wall (Ryu et al., 2004; Arroyo et al., 2012) (Figure 1.2). Furthermore, the absorption and tissue distribution of cadmium has been shown to be mediated by a channel-like calcium transport (CaT1, encoded by the gene TRPV6) which is used for intestinal absorption of calcium (Min et al., 2008).

Once absorbed through the intestinal tract, cadmium readily binds albumin and is delivered by portal circulation to the liver. The sinusoidal capillaries responsible for the uptake of cadmium into hepatocytes have membranes lined with DMT-1 as well as Zrt-, Irt-related proteins (ZIP) 8 and 14 (encoded by the genes SLC39A8 and SLC39A14, respectively), which are involved in zinc and iron transport, respectively (Fujishiro et al., 2009; Liuzzi et al., 2006). Once inside hepatocytes, cadmium avidly binds to proteins containing sulfhydryl groups including glutathione (GSH) and with high affinity for metallothionein (MT). As an 


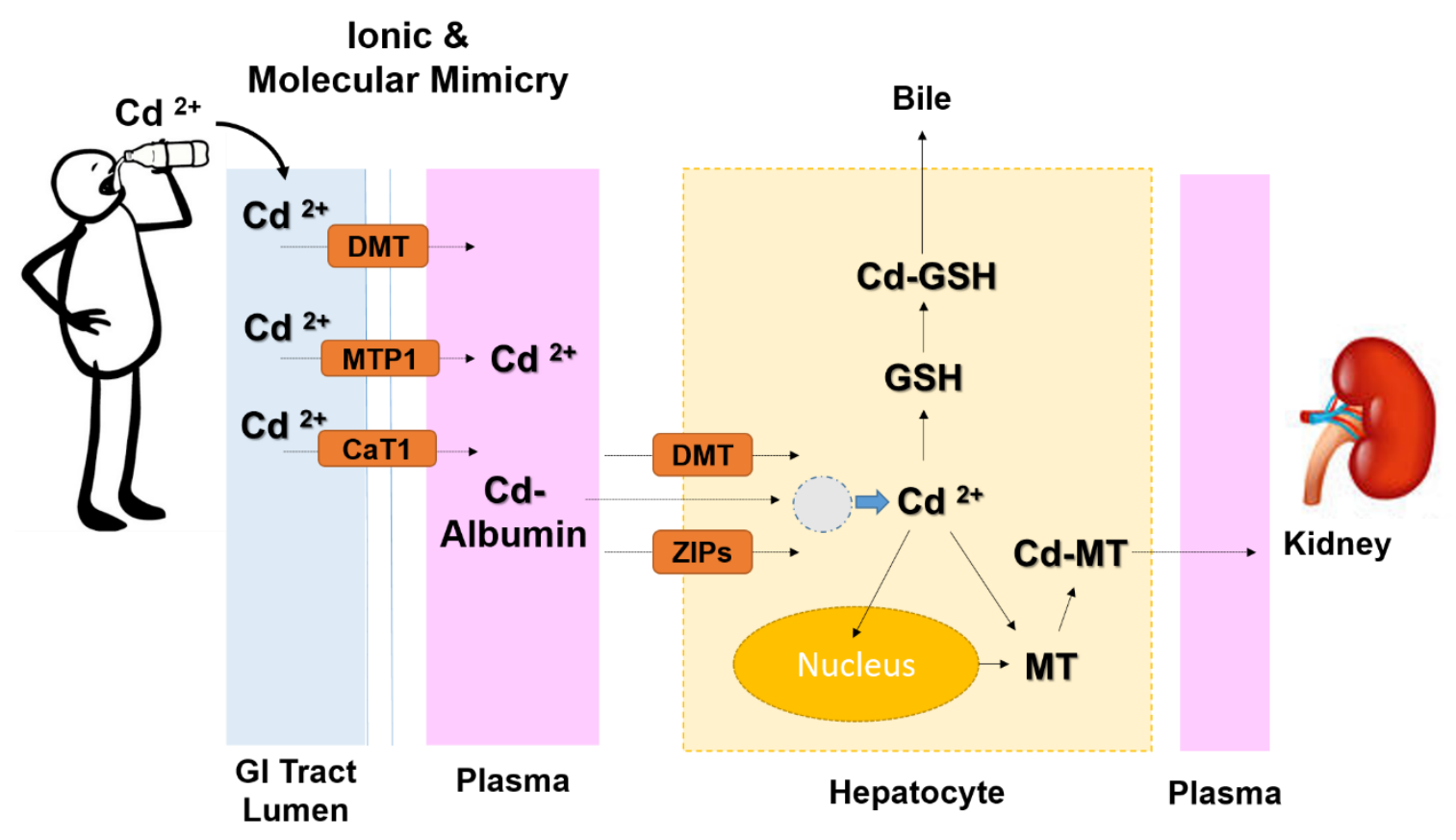

Figure 1.2. Cadmium disposition after oral exposure. Simplified schematic of cadmium absorption and distribution via ionic and molecular mimicry to the liver and kidney, the target organ of cadmium accumulation and toxicity, after oral ingestion. $\mathrm{Cd}=$ cadmium, $\mathrm{DMT}=$ divalent metal transporter, $\mathrm{MTP}=$ metal transport protein 1, CaT1 $=$ channel-like calcium transport 1, ZIPs = Zrt-, Irt-related proteins, $\mathrm{Gl}$ = gastrointestinal, $\mathrm{MT}=$ metallothionein, $\mathrm{GSH}=$ glutathione . 
initial step in the detoxification process, cadmium binds to GSH and is excreted into the bile via the canalicular glutathione transport system where it is mainly excreted into the feces (Kurowska and Bal, 2010). However, cadmium has a strong binding affinity for MT, which it preferentially binds to in the liver, serving as both a method of detoxification and tissue retention, playing a key role in cadmium's long biological half-life ( 20 years) in the liver (ATSDR, 2012; Arroyo et al., 2012).

In scenarios of chronic cadmium exposure, the cadmium bound MT complexes formed in the liver are released from damaged hepatocytes into the blood stream (Klassen et al., 2009). The cadmium-MT complexes are filtered by the kidney, and subsequently reabsorbed into the renal proximal tubule cells. Cadmium is then eliminated from the body in urine at a very slow rate thus contributing to its long half-life. In addition, urinary cadmium levels are used as markers of chronic cadmium exposures whereas blood cadmium levels are considered to be more an indication of acute exposures (Vacchi-Suzzi et al., 2016). The majority of toxicological research has focused on cadmium's role as a nephrotoxin and carcinogen. However, more recent studies show exposure to low level, environmental cadmium correlates with the development of several metabolic diseases, including type 2 diabetes, hypertension and NAFLD (Hyder et al., 2013 ; Padilla et al., 2010; Little et al., 2020).

As a major site of cadmium accumulation, it is not surprising that the liver is a target organ of cadmium toxicity. In animal models of liver failure, cadmium exposure is well known to induce inflammation, apoptosis and liver cell regeneration (Habeebu et al., 2000). These models, however, were designed to 
investigate the impacts of cadmium at high acute exposures, and did not take into consideration the impact of lower, environmental exposures on liver disease. As such, more recent in vivo studies have revealed low dose exposure to cadmium dysregulates NAFLD-related metabolic pathways, disrupts essential metal homeostasis and increases liver and serum triglycerides (Go et al., 2015; Zhang et al., 2015; Young et al., 2019). In addition, environmental cadmium exposure has been associated with hepatic necroinflammation, NAFLD and NASH in men and necroinflammation women (Hyder et al., 2013). Despite these research advances, our understanding of cadmium's impact on the development and progression of NAFLD is insufficient and unclear.

Our understanding of the impact of cadmium exposure on obesity is also unclear. Epidemiologically, the existing data associating cadmium exposure with obesity are contradictory (Tinkov et al., 2017). In particular the existing data vary between positive (Akinloye et al., 2010; Padilla et al., 2010) and negative associations (Tellez-Plaza et al., 2013; Nie et al., 2016) with some studies showing no significant impact (Kelishadi et al., 2013; Gonzalez-Reimers et al., 2014). The inconsistencies in the epidemiological findings may in part be explained by differences in the levels of cadmium exposure, variability in analytical methods and the use of different biological markers (i.e. nails, blood, urine, hair).

Results from laboratory studies are more consistent, showing that cadmium exposure alone does not result in obesity but does adversely affect adipose tissue physiology (i.e. decreased leptin, adiponectin, and insulin receptor number and density) (Levy et al., 2000; Fickova et al., 2003; Kawakami et al., 2013). The 
altered adipose tissue physiology is speculated to increase the predisposition to metabolic diseases, such as NAFLD, through increased insulin resistance and an overall induction of a metabolic profile consistent with obesity (Simmons et al., 2014; Tinkov et al., 2017).

Although it is well known that consumption of a high fat diet contributes greatly to obesity, few studies have directly investigated the relationship between cadmium and high fat diet in disease initiation and progression. Zhang et al. (2020) showed combined exposure to low levels of cadmium and high fat diet adversely affected bone quality. Another study showed a combination of high cholesterol diet and cadmium resulted in cardiac fibrosis and increased risk of heart failure (Türkcan et al., 2015). Our lab has recently shown that cadmium and high fat diet work together to further disrupt essential metal homeostasis (Young et al., 2019). Although informative, these studies highlight major knowledge gaps in our understating of the effects of cadmium on high fat diet induced-diseases, including NAFLD.

\section{Sex (a third hit)}

As previously mentioned and according to the "multiple hit" hypothesis, NAFLD progression is determined by multiple factors acting in parallel. In addition to diet and environmental exposures such as cadmium, recent attention was turned to sex as a biological variable and risk factor. In fact, NAFLD is a sexually dimorphic disease, occurring more often and with greater severity in men than in women (Hyder et al., 2013). These results have been recapitulated in animal 
studies (Lonardo et al., 2019). However, little attention is given to the sexualdimorphism of the multiple risk factors involved in disease etiology. For example, in addition to being a major risk factor for NAFLD, obesity itself is also a sexually dimorphic disease from which females are more protected than males, at least before menopause (Pettersson et al., 2012; Palmer and Clegg, 2015; Salinero et al., 2018). After menopause, the prevalence and severity of obesity becomes greater in women (Hales et al., 2020).

These epidemiological outcomes were recently recapitulated in an in vivo study by Salinero et al. (2018). This study showed high fat diet-induced obesity in juvenile mice (administered at 6 weeks of age for 12 weeks) increased weight gain and cause glucose intolerance to a greater extent in males compared to females. However, in an aged mouse (high fat diet administered at 32 weeks of age for 12 weeks), the sex differences were reversed with greater weight gain and glucose impairment in female mice. The mechanism for age-dependent sex differences in obesity are largely thought to be the result of hormones (Salinero et al. 2018); however, further investigation is needed to understand the sex-dependent influence of these hormones on diet-induced diseases, including NAFLD.

Sex-dependent differences also occur in response to environmental stress, such as exposure to metals. There is increasing evidence that the adverse health effects associated with exposure to toxic metals manifest differently in women and men due to variation in exposure, kinetics, mode of action (Vahter et al., 2007). In the case of cadmium exposure, the body burden of the metal is much greater in women compared to men. Based on reports from occupational, high dose 
exposures, adverse health effects associated with cadmium are more common in women; however these reports are generally in post-menopausal, elderly women (Wang et al., 2003; Vahter et al., 2007; Akesson et al., 2005). In a more recent study, Hyder et al. (2013) showed that lower, environmental exposure to cadmium was associated with necroinflammation, NAFLD and NASH in men and only necroinflammation in women of reproductive age. Although there is clear evidence for sex differences in cadmium disposition and toxicity, the underlying mechanisms remain unclear. Furthermore, how sex influences the multiple factors (diet and environmental exposures) associated with NAFLD progression, in parallel, remains to be investigated.

In fact, the effect of sex on the combined exposure of cadmium and high fat diet have only been reported by our lab. Liang et al (2019) showed exposure to low doses of cadmium ( 0.5 and 5 ppm in drinking water) combined with postweaning high fat diet for 10 weeks resulted in exacerbated high fat diet-induced heart hypertrophy in female mice, but not male mice. Using the same model, we reported cadmium exposure combined with post-weaning high fat diet for 24 weeks altered essential metal homeostasis in the liver and kidney (Young et al., 2019). Additionally, we found significant cadmium- and high fat diet-dependent sex differences in essential metal distribution, which may contribute to differences observed in obesity-associated pathologies between males and females. The results from these two studies lay the foundation for future studies investigating how sex, diet and environmental exposure work in parallel to influence disease progression. 


\section{Developmental Origins of Health and Disease (an important factor)}

In relation to the "multiple hit" hypothesis, another factor contributing to disease development and progression is that of chronicity and the timing. As such, the focus of developmental toxicology has shifted from studying the effects of toxicant-induced malformations to understanding how non-overtly toxic exposures, in utero, result in increased susceptibility to diseases in adulthood. A variety of terms have been used to describe this novel area of research including "fetal origins of disease", "fetal beginnings of adult disease", "fetal basis of adult disease" and the "developmental origins of adult disease". However, although not established as official terminology, the term Developmental Origins of Health and Disease (DOHaD) is most commonly used (Barker, 2007; Heindel et al., 2017). Originating in the 1990s, the $\mathrm{DOHaD}$ hypothesis suggests that exposures to environmental stressors during sensitive stages of human development (in utero and early childhood) increases susceptibility to adverse health outcomes in adulthood (Barouku et al., 2012; Gluckman et al., 2010; Thayer et al., 2012).

The Dutch famine is one of the earliest documented examples of prenatal environmental factors being associated with disease development in adulthood (Painter et al., 2005). Toward the end of WWII, during the winter of 1944-1945, the Dutch government implemented a railway strike with the intent of halting transport of German troops and reinforcements, thereby aiding the Allied Forces. In retaliation, the German government cut off food and fuel shipments to the Germanoccupied areas of the western Netherlands. As a result, the daily rations for the general adult population dropped from 1800 calories to $<1000$ calories/day. From 
this unfortunate event came the Dutch famine birth cohort study (Painter et al., 2005). Although the offspring of women who were pregnant during the famine were small for gestational age, in adulthood this cohort had an increased incidence of obesity (Ravelli et al., 1999), diabetes (de Rooij et al., 2006; van Abeelen et al., 2012), and cardiovascular disease (CVD) (Roseboom et al., 2000).

In the decades following the Dutch famine there have been numerous studies, performed in both humans and animals, that suggest that in utero exposures to environmental stressors, such as poor nutrition or exposure to environmental chemicals, contribute to the initiation, and/or exacerbation of chronic adult diseases such as CVD (Barker and Osmond, 1986; Barker et al., 1989; Barker et al., 1993) and cancer (Newbold and McLachlan, 1996; Waalkes et al., 2003). In 1986, Barker and Osmond observed that the same regions in England with high rates of low-birthweight induced infant mortality had the highest rates of death associated with coronary artery disease decades later (Barker and Osmond, 1986). It is this observation that initiated the "fetal basis for adult disease" hypothesis, also known as the "Barker Hypothesis".

Although many of the supporting studies that followed Barker's observation were focused on the effects of altered nutrition in utero, there has been growing evidence that other factors including sex and environmental exposures increase susceptibility to the development of chronic adult diseases (Heindel et al., 2015; Jazwiec and Sloboda, 2019), such as NAFLD. In fact, in models of maternal high fat diet feeding, adverse outcomes, such as increased hepatic triglycerides and de novo lipogenesis, are more prominent in male offspring than female offspring and 
lead to the development of NAFLD in male, but not female offspring (Baylo et al., 2010; Strakovsky et al., 2014; Li et al., 2015).

The influences of sex, as a biological factor, on the DOHaD hypothesis is become increasingly recognized. Stress, nutritional insults, and environmental chemical exposures during development can result in sex-specific effects (Heindel et al., 2015; Jazwiec and Sloboda, 2019). The long term impacts of adversity in early life are largely thought to operate through epigenetic mechanisms such as altered DNA methylation patterns, which also varies between males and females (Martin and Fry, 2018). For example, there are baseline differences in the methylation patterns of the placentas from male and female pregnancies (Martin et al., 2017). Many of these baseline differences are associated with transport and metabolism of environmental contaminants and thus may provide valuable insight into sex-dependent differences observed in environmental contaminantassociated health effects. Indeed, sex-specific differences in methylation patterns associated have been observed with a number of environmental contaminants, including cadmium (Huen et al., 2014; Alegría-Torres et al., 2016; Kippler et al., 2013).

Due to the widespread production and use of metals, in parallel with the knowledge that metals can be passed from mothers to offspring via placenta and/or breast milk, concern has risen about prenatal exposure to metals and the long-term adverse health implications (Wang et al., 2014; Young et al., 2018). For some metals, such as arsenic, the connection between in utero exposures and increased risk of disease development in adulthood, including NAFLD, is evident 
in both human and animal studies (Young et al., 2018); however, for other metals, such as cadmium, this connection remains unclear.

In adults, the association between chronic cadmium exposures and development of adverse health effects such as renal toxicity, CVD and cancer is well documented (Jarup and Akesson, 2009; Nawrot et al., 2010). In children, preand postnatal exposure to cadmium is associated with reduced birthweight, impaired fetal growth, trace element deficiencies and congenital malformations (AlSaleh et al., 2014; Hudson et al., 2019; Jin et al., 2016; Kippler et al., 2012; Taylor et al., 2016). Although it is well documented that prenatal exposure to cadmium is associated with adverse effects on child health and development, the implication for long term health remain elusive (Figure 1.3). Few studies have considered the possibility that prenatal cadmium exposure may reprogram an individual to be more susceptible to pathologies later in life.

Epidemiologically, the link between early-life cadmium exposures and increased risk for adult diseases remains to be explored. Experimentally, researchers have just begun to develop models to study the impact of early life cadmium exposure on disease development later in life. Hudson et al. (2019) investigated the impact of maternal cadmium exposure on cardiovascular changes in offspring at birth or after 6 months of age. This study showed maternal cadmium exposure was associated with a hypertensive phenotype in adult female, but not in male mice (Hudson et al., 2019). Furthermore, as mentioned above, our lab has reported whole life exposure to cadmium, starting in utero, enhances diet-induced cardiac hypertrophy in female mice as well as disrupts essential metal 


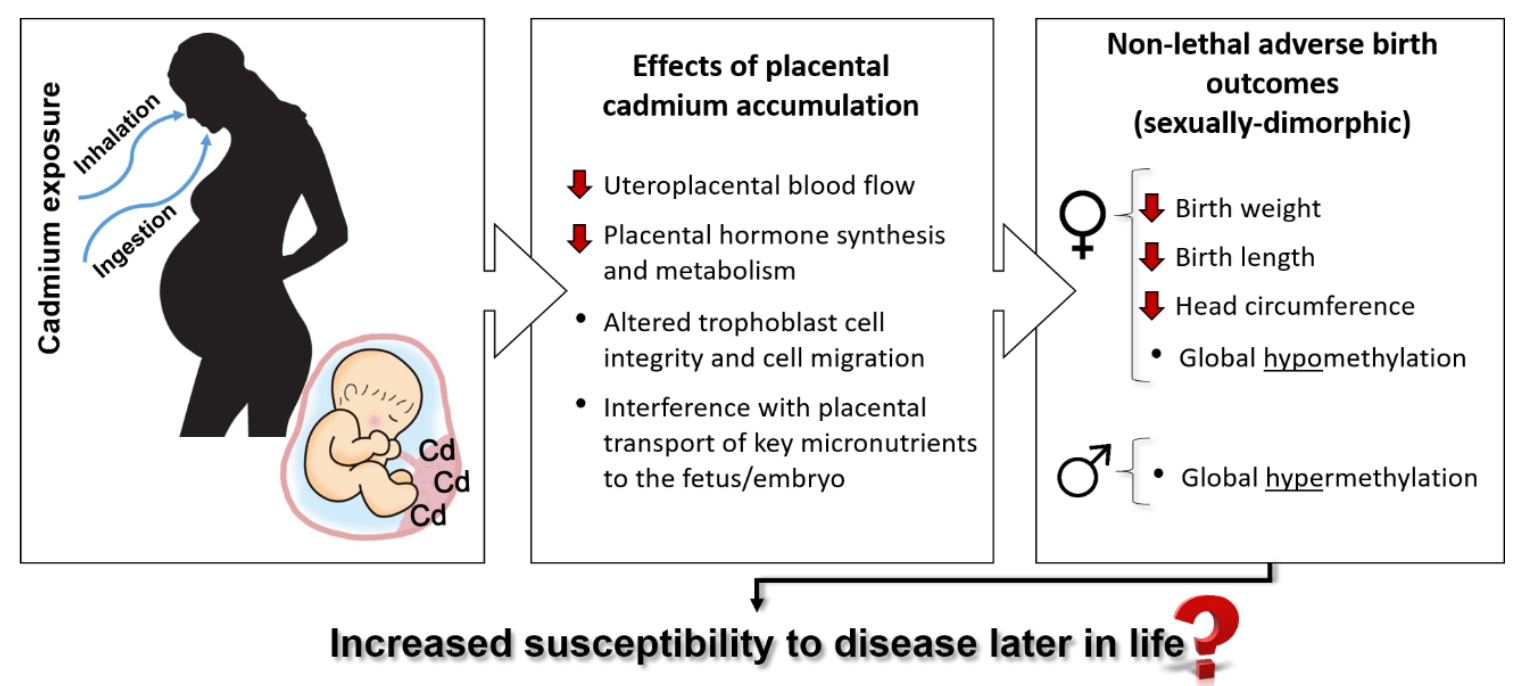

Figure 1.3. Summary of the effects of placental cadmium accumulation and the non-lethal adverse birth outcomes that may lead to increased susceptibility of disease development in adulthood (Young and Cai, 2020). 
homeostasis in a sex-specific manner (Liang et al., 2019; Young et al., 2019). While results from these studies provide critical insight into the importance of early life exposure to cadmium on the development of disease later in life, empirical evidence is still needed to strengthen these observations in both animal models and prospective birth cohort studies in humans. Additionally the impact of early life cadmium exposure on the development of high fat diet-induced NAFLD remains unexplored.

\section{Summary and dissertation aims}

The purpose of this dissertation is address these key data gaps and investigate the impact of whole life, low-dose cadmium exposure on high fat dietinduced non-alcoholic fatty liver disease (NAFLD). NAFLD affects more than $20 \%$ of the adult population in the United States (Younossi et al., 2018). Although obesity is a major risk for the development of NAFLD, it does not account for all cases (Younossi et al., 2018). Thus, other risk factors, including genetics and/or environmental exposures, play an important role. Previous studies have correlated environmental exposures to the nonessential metal cadmium with the development of NAFLD (Padilla et al., 2010). However, the mechanisms by which cadmium influences the initiation, progression and exacerbation of NAFLD induced by high fat diet are not well understood. In addition, these studies do not consider the fact that environmental exposures can be life-long and multigenerational nor do they take into account that the risk of developing 
adulthood disease is correlated with adverse stimuli in utero, resulting in permanent physiological and metabolic changes.

Therefore, we developed a multi-hit model, considering diet, sex and environmental exposure, to study the effects of whole life exposure to low dose cadmium on the development of high fat diet-induced NAFLD. Our central hypothesis is that whole life, chronic cadmium exposure exacerbates high fat dietinduced NAFLD in males more than females. This hypothesis will be tested with three Specific Aims:

Aim 1: Measure the impact of whole-life, chronic cadmium exposure on HFDinduced NAFLD.

In this aim, a mouse model of cadmium exposure and high fat diet induced NAFLD will be established and used to test the central hypothesis. Exposure of parental mice will be for 18 weeks. Male offspring will be exposed to cadmium in utero and, after weaning, continued on normal or high fat diet, with or without cadmium for 24 weeks. The model will be used to assess key endpoints reflecting metabolic alterations and hepatic toxicity, characteristic of NAFLD at the whole animal, tissue and molecular levels.

Aim 2. Show zinc supplementation protects against cadmium-enhanced, high fat diet-induced NAFLD.

Our lab showed zinc supplementation rescues cardio-renal hypertrophy in high fat diet-fed mice (Wang et al., 2017). Others showed zinc supplementation 
attenuates high fat diet-induced NAFLD in rats (Shidfar et al., 2018). Therefore, this aim will examine dietary zinc supplementation as a treatment approach and test the hypothesis that zinc supplementation protects against cadmiumexacerbated, high fat diet-induced NAFLD. This aim will be investigated using the same exposure paradigm established in the first aim. However, after weaning male offspring will be further divided into normal diet and high fat diet groups receiving either normal zinc or supplement zinc in their diets. At the whole animal, tissue and molecular levels the impact of altered dietary zinc on key endpoints characteristic of NAFLD will be assessed.

Aim 3. Determine the role of sex in cadmium-enhanced, high fat diet-induced NAFLD.

The prevalence and manifestation of numerous metabolic diseases are sexually-dimorphic, including NAFLD (Ballestri et al., 2017; Lonardo et al., 2019). Additionally, there is increasing evidence that the health effects of toxic metals, including cadmium, are different between men and women (Vahter et al., 2007). We recently showed that low-dose cadmium exposure induces cardiac hypertrophy and fibrosis in female but not male mice (Liang et al 2019). Therefore, this aim will examine sex as a risk factor and test the hypothesis that female mice are protected against cadmium-enhanced, high fat diet-induced NAFLD. A female mouse model of NAFLD and cadmium exposure will be established, mirroring the model created in the first aim. The model will test the hypothesis that female sex protects against cadmium enhanced, high fat diet-induced NAFLD. 


\section{CHAPTER 2: MATERIALS AND METHODS}

This section contains the materials and methods for all three dissertation aims.

\section{Animal models and exposures}

Eight week old male $(n=19)$ and female $(n=51)$ C57BL/6J mice were purchased from Jackson Laboratory (Bar Harbor, ME). Mice were maintained on a 12 hour light/dark cycle at $25^{\circ} \mathrm{C}$ in a pathogen-free barrier facility accredited by the Association for Assessment and Accreditation of Laboratory Animal Care. All experimental procedures were approved by the University of Louisville's Institutional Animal Care and Use Committee. After one week of acclimation in the barrier facility, diets were switched from standard laboratory chow to purified normal mouse diet (ND, 10\% kcal fat; Research Diets D14020202, New Brunswick, $\mathrm{NJ}$ ) to minimize the influence of metal contamination found in standard laboratory chow on experimental outcomes (Kozul et al., 2008). At 9 weeks old, mice were placed on a drinking water regimen of either tap water alone (control) or cadmium containing water ( 0.5 or $5 \mathrm{ppm}$ - final concentration). Cadmium containing drinking water was prepared weekly from stock solutions of cadmium chloride (Alfa Aesar, Haverhill, MA) made with deionized water and stored at $-80^{\circ} \mathrm{C}$. Five ppm cadmium was used as a positive control because based on a 
survey of the literature, $5 \mathrm{ppm}$ cadmium is one of the lower concentrations of cadmium tested with results supportive of metabolic syndrome phenotypes. In addition we used a ten times lower concentration of $0.5 \mathrm{ppm}$ cadmium. According to the Agency for Toxic Substances and Disease Registry (ATSDR) approximately 100 to $300 \mathrm{mg} / \mathrm{kg}$ cadmium ( $1 \mathrm{ppm}=1 \mathrm{mg} / \mathrm{kg}$ ) is lethal to $50 \%$ of rodents after acute oral exposure (ATSDR, 2012). Our oral exposures are less than $1 \%$ and $0.1 \%$ of this cadmium level.

At 10 weeks of age, mice were placed into mating groups $\left(F_{0}\right)(1$ male to 2 or 3 females) within each drinking water exposure (Figure 2.1). Pregnant dams continued on the drinking water regimen through weaning and offspring $\left(F_{1}\right)$ were continuously exposed to the same concentration of cadmium as their parents after weaning. At weaning, offspring were either fed the same ND as their parents or a high fat diet (HFD, 60\% kcal fat; Research Diets D14020205, New Brunswick, NJ). Male mice were further grouped into diets containing 30 or $90 \mathrm{mg} \mathrm{zinc/4057} \mathrm{kcal,}$ representing normal zinc (ZN) and zinc supplemented (ZS) diet, respectively. (ND/ZS - Research Diets D14020203; Research Diets D14020206, New Brunswick, NJ).

Food and deionized water were provided ad libitum. Body weights and water consumption was recorded weekly. First litter animals were too few; therefore, they were sacrificed and tissues stored for other possible studies. The male and female parents $\left(F_{0}\right)$ who had been continuously exposed to cadmium in their drinking water for 14 weeks (through the first mating and through the first 


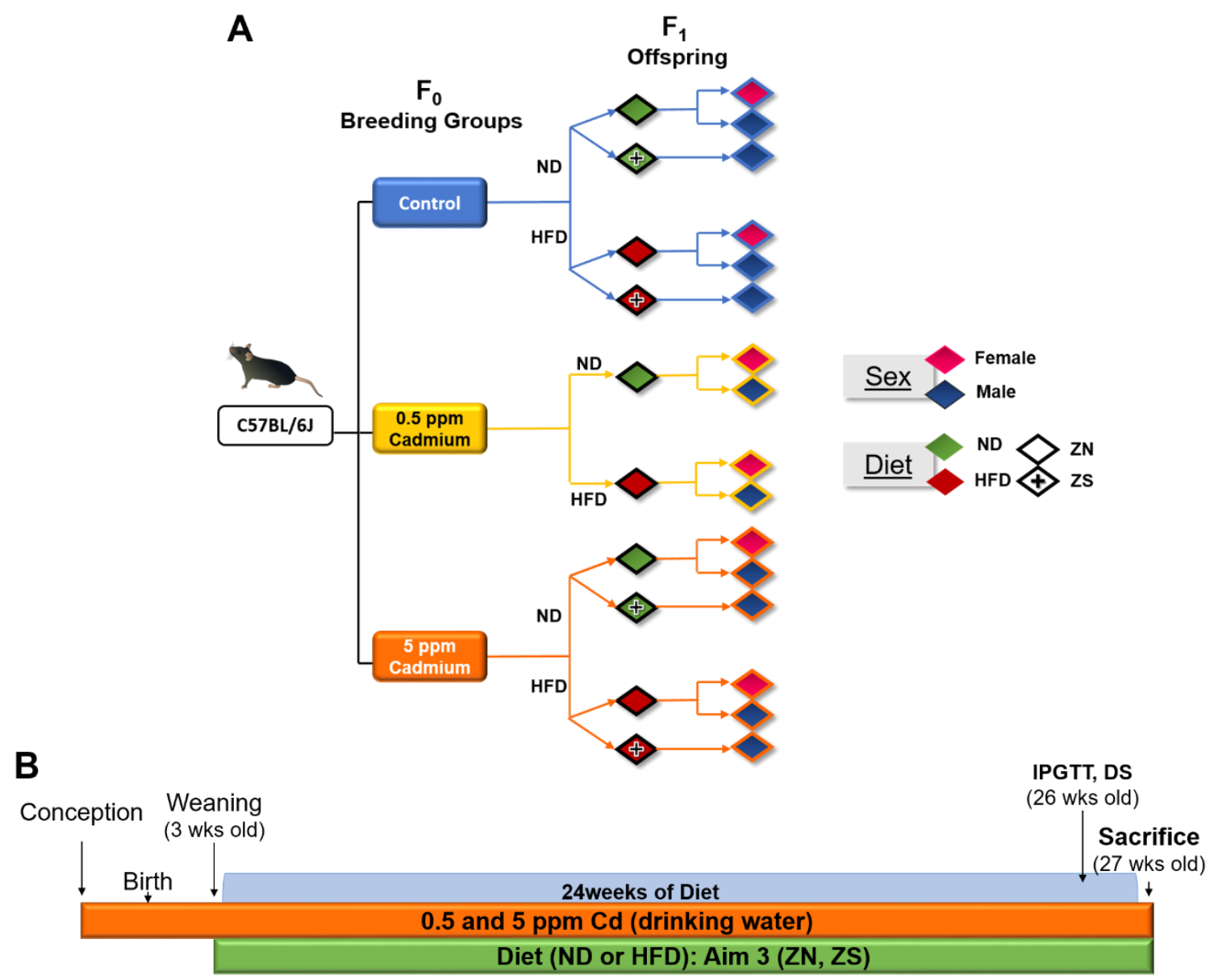

Figure 2.1. Exposure paradigm. (A) Adult male and female C57BL/6J mice on defined, diets were exposed to control drinking water, or water containing 0.5 or 5 ppm Cd for 14 weeks before being established into breeding pairs. Pregnant dams and offspring were continuously exposed and continued on the same drinking water regime as their parents after weaning. At weaning, offspring were also fed either a normal or high-fat diet (ND or HFD, respectively) for 24 weeks. The same paradigm was used in Aim 2 with the modulation of zinc in the diet. (B) One week prior to sacrifice, IPGTT tests were performed and body composition determine by DEXAscan (DS). IPGTT = intra-peritoneal glucose tolerance test, wks = weeks, ZN = zinc normal diet, ZS = zinc supplemented diet. 
pregnancy) were placed into mating groups again ( 1 male to 2 or 3 females) to produce a second litter. These second litter offspring were subjected to the same exposure paradigm as the first litter and euthanized 24 weeks post-weaning (details below).

Mice were anesthetized with an intraperitoneal injection of avertin (250 $\mathrm{mg} / \mathrm{kg}$ ). Blood was collected from the inferior vena cava prior to euthanasia via exsanguination. Blood samples were centrifuged for 5 minutes at 6,000 rpm and citrated plasma was stored at $-80^{\circ} \mathrm{C}$ for further biochemical analysis. For each mouse, liver weight was recorded and portions of liver tissue were snap-frozen in liquid nitrogen, processed for RNA isolation, fixed in $10 \%$ neutral buffered formalin for histology and immunohistochemistry, frozen-fixed in Tissue Tek OCTCompound (Sakura Finetek, Torrance, CA) or used for metals analysis. The liver weight to tibia length ratio (liver weight in grams divided by tibia length in millimeters) was used as an index of liver size changes.

\section{Body composition}

Although body mass index (BMI) continues to be the most widely used measurement of obesity, it is a poor assessment of metabolic status and does not take into consideration lean tissue mass (muscle mass), bone mineral content nor distribution of fat mass (Heymsfield and Cefalu, 2013; Ponti et al., 2019). Therefore, we used dual-energy X-ray absorptiometry scan (DEXAsan) technology to more accurately and informatively assess body mass composition changes in our animal model. A Lunar PIXImus Densitometer (GE Healthcare, Chicago, IL), 
provided by the University of Louisville Diabetes and Obesity Center Animal Models and Phenotyping Core, was used to perform DEXAscans on randomly selected mice from each exposure group. Briefly, mice were weighed then anesthetized with $2-4 \%$ isoflurane in $\mathrm{O}_{2}$ by inhalation. The depth of anesthesia was determined by response to toe pinch. Once under adequate anesthesia, the mouse was placed on a specimen tray on the scanning platform with legs slightly outstretched, tail curled around the left side of the mouse, straight spinal alignment and head positioned to fit the nose within the nosecone (continued provision of anesthesia). After ensuring the limbs and body of the mouse were in the predetermined outlines on the specimen tray, the animal ID, body weight, gender and date of birth were entered into the software and the scan started (4-5 minutes). At the end of the scan, mice were taken off the platform and placed into a "recovery cage" and monitored. Using the PIXImus software, a manual analysis was performed to adjust the region of interest (ROI) to exclude the head region, the scan repositioned and data exported. Data output included body composition (\% fat), lean tissue mass $(\mathrm{g})$, bone mineral density $(\mathrm{BMD})\left(\mathrm{g} / \mathrm{cm}^{2}\right)$ and bone mineral content (BMC) (mg).

\section{Glucose tolerance test}

The intraperitoneal glucose tolerance test (IPGTT) measures the body's ability to clear an injected load of glucose. Dysregulation of glucose metabolism is associated with the pathophysiology of many metabolic disorders, including NAFLD (Chao et al., 2019). Mice were fasted for 6 hours, weighed and injected 
with a $2 \mathrm{~g} / \mathrm{kg}$ glucose solution. Blood glucose levels were measured at 0 (preinjection), 15, 30, 60, and 120 minutes post-glucose injection using a FreeStyle complete blood glucose monitor system (Abbott Diabetes Care Inc., Alameda, CA). A time course of absolute blood glucose measurement and the area under the curve $(A \cup C)$ were determined for each animal.

\section{Biochemical analysis}

Plasma insulin levels were measured with the Ultra Sensitive Mouse Insulin ELISA Kit (Crystal Chem, Elk Grove Village, IL) using the manufacturer's instructions for preparing samples and reagents for the wide range assay $(0.1$ $12.8 \mathrm{ng} / \mathrm{mL})$.

Alanine aminotransferase (ALT) and aspartate aminotransferase (AST) are released into the blood stream as a result of injury to the liver and are thus used as clinical biomarkers of hepatic injury. Levels of plasma ALT and AST were determined spectrophotometrically using Infinity ${ }^{\mathrm{TM}}$ ALT (GPT) and AST (GPO) Liquid Stable Reagents (Thermo Fisher Scientific, Waltham, MA) per the manufacturer's instructions. Data Trol ${ }^{\mathrm{TM}}$ Normal and Abnormal Control Serum were used as assay controls (Thermo Fisher Scientific, Waltham, MA).

Hepatic triglycerides (TG) were determined spectrophotometrically using Infinity ${ }^{\mathrm{TM}}$ Triglycerides Liquid Stable Reagents (Thermo Fisher Scientific, Waltham, MA) per the manufacturer's instructions. A $200 \mathrm{mg} / \mathrm{dL}$ TG standard was used as an assay control (Thermo Fisher Scientific, Waltham, MA). Liver tissue (70-100 mg) was homogenized in a $2 \mathrm{~mL}$, flat bottom microcentrifuge tube, on ice 
in $1 \mathrm{~mL}$ of $50 \mathrm{mM}$ sodium chloride. For each sample, $500 \mu \mathrm{l}$ of homogenate was placed into a $15 \mathrm{~mL}$ conical tube with $4 \mathrm{~mL}$ of fresh extraction buffer (2 parts Chloroform: 1 part Methanol) and vortexed for 2 minutes at room temperature. Samples were then centrifuged at room temperature for 20 minutes at $1800 \times g$. The volume of the clear, lower chloroform layer was recorded and $500 \mu \mathrm{L}$ of the chloroform layer was placed into a $2 \mathrm{~mL}$, flat-bottom microcentrifuge tube. Samples were placed in a chemical hood, uncapped, and allowed to evaporate overnight. The next day, $500 \mu \mathrm{L}$ of Infinity ${ }^{\mathrm{TM}}$ Triglycerides Liquid Stable Reagent (equilibrated to room temperature) was added to each of the dried samples and vortexed for at least 2 minutes. Samples were then incubated for 10 minutes at $37^{\circ} \mathrm{C}$. At the end of incubation, $200 \mu \mathrm{L}$ of sample/reagent mix was added in duplicate to a 96 well microplate and total TG measured with a plate reader at $500 \mathrm{~nm}$.

Total hepatic cholesterol (TC) was determined spectrophotometrically using Infinity ${ }^{\mathrm{TM}}$ Cholesterol Liquid Stable Reagents (Thermo Fisher Scientific, Waltham, MA) per the manufacturer's instructions. A $200 \mathrm{mg} / \mathrm{dL}$ TC standard was used as an assay control (Thermo Fisher Scientific, Waltham, MA). Liver samples were extracted and analyzed as described above for total hepatic triglycerides.

\section{Histological analysis}

Paraffin-embedded sections of liver $(5 \mu \mathrm{m})$ were deparaffinized with xylene, rehydrated with graded ethanol washes and stained with hematoxylin and eosin (H\&E) to assess overall hepatic structure. Pictures were taken on an Olympus BX43 microscopy system using Olympus cellSense Imaging Software (Shinjuku 
City, Tokyo, Japan). Steatosis was scored as percent of liver cells in a 100x field containing fat $(<25 \%=1+;<50 \%=2+;<75=3+;>75 \%=4+)$. For each animal, ten 100x fields were scored. Histological evaluation of neutral lipids was done using oil red O (ORO) (C26H24N4O), a fat soluble, diazole dye (Sigma Aldrich, St. Louis, MO) (Mehlem et al., 2013). Fresh frozen liver sections $(10 \mu \mathrm{m})$ were fixed in $10 \%$ neutral buffered formalin for 20 minutes, rinsed with $\mathrm{ddH}_{2} \mathrm{O}$, immersed in freshly prepared $60 \%$ isopropanol for 2 minutes and stained with freshly prepared ORO working solution (6 parts saturated Oil Red O isopropanol solution : 4 parts $\mathrm{ddH}_{2} \mathrm{O}$ ) for 30 minutes at room temperature. ORO stained slides were then rinsed with $\mathrm{ddH}_{2} \mathrm{O}$ and lightly stained with hematoxylin (10 dips), rinsed with distilled water (10 dips), and rinsed (10 dips) in 3\% acetic acid to remove access hematoxylin. Slides were rinsed for 10 minutes in running tap water and mounted with glycerol. The same day, ten 100x pictures were taken on an Olympus BX43 microscopy system using Olympus cellSense Imaging Software (Shinjuku City, Tokyo, Japan). Exposure time and gain settings were determined using a sample known to have excessive ORO staining. Image J software (National Institutes of Health, Bethesda, Maryland) was used to quantify the percent of area positively stained with ORO.

\section{Total RNA isolation, cDNA synthesis and qRT-PCR}

Total RNA was isolated from liver tissues using STAT 60 (Amsbio LLC, Abingdon, United Kingdom), following the manufacturer's protocol for RNA isolation. Briefly, tissue was homogenized on ice in $1 \mathrm{~mL}$ of STAT 60 reagent and 
allowed to sit at room temperature for 5 minutes before the addition of chloroform. Sample homogenates were vortexed for 15 seconds, allowed to sit at room temperature for 2 minutes, and centrifuged at $12,000 \mathrm{~g}$ for 15 minutes at $4^{\circ} \mathrm{C}$. The aqueous phase was transferred to a fresh tube, mixed with isopropanol, allowed to sit at room temperature for 8 minutes and centrifuged at $12,000 \times g$ for 10 minutes at $4^{\circ} \mathrm{C}$. After centrifugation, the supernatant was carefully removed, not disturbing the RNA precipitate on the bottom of the tube. The pellet was washed with $75 \%$ ethanol and allowed to air dry in a chemical fume hood followed by the addition of diethylpyrocarbonate (DEPC) treated RNase-free water to solubilize the RNA. The quality and the concentration of the RNA was measured using a NanoDrop ND-1000 spectrophotometer.

Using a Reverse Transcription System (Promega, Madison, WI), 1 $\mathrm{gg}$ of total RNA was used to synthesize cDNA following the manufacturer's instructions with slight modifications. For each sample, a $20 \mu \mathrm{L}$ reaction was prepared by combining 10 mM dNTP mixture, Recombinant RNasin® Ribonuclease Inhibitor, AMV Reverse Transcriptase, random primers and nuclease-free water with $1 \mu \mathrm{g}$ total RNA. For each reverse transcriptase reaction a no RNA control and a no reverse transcriptase control were included. Quantitative RT-PCR reactions were performed using TaqMan® RNA assays (Thermo Fisher Scientific, Waltham, MA). TaqMan gene expression assays (GAPDH, Mm99999915_g1; MT1, Mm00496660_g1; MT2, Mm04207591_g1; SCD-11, Mm00772290_m1; FASN, Mm00662319_m1; SREBF1, Mm00550338_m1; HMGCR, Mm01282499; HMGCS, Mm01304569_m1) were combined with Entrans 2x qPCR Probe Master 
Mix (ABclonal, Woburn, MA) and DEPC water to create a master mix for each primer. The master mix was added in duplicate to a 96 well plate, followed by the addition of the corresponding cDNA. For all qRT-PCR runs a no cDNA control was included in addition to the no reverse transcriptase and no RNA controls produced during cDNA synthesis. Reactions were performed on a LightCycler® 96 RealTime PCR Cycler (Roche, Basel, Switzerland) using the following conditions. Samples were warmed up at $4.4^{\circ} \mathrm{C} /$ second until they reached $95^{\circ} \mathrm{C}$ at which point they were held for 10 minutes, followed by 45 cycles of denaturing ( 10 seconds at $95^{\circ} \mathrm{C}$ ) and annealing/extending ( 45 seconds at $60^{\circ} \mathrm{C}$ ). Results are presented as $\Delta \Delta \mathrm{Ct}$ values relative to unexposed, normal diet controls.

\section{Protein extraction for western blot}

Liver tissues (approximately $30 \mathrm{mg} / \mathrm{liver}$ ) were homogenized on ice in lysis buffer containing $50 \mathrm{mM}$ Tris- $\mathrm{HCl}(\mathrm{pH} 7.4), 1 \mathrm{mM} 0.5$ EDTA $(\mathrm{pH} 8.0,50 \mathrm{ug} / \mathrm{mL}$ phenylmethylsulfonyl fluoride and Protease Inhibitor Cocktail (Sigma-Aldrich, St. Louis, MO). After homogenization samples were placed on a rotating mixer for 4 hours at $4^{\circ} \mathrm{C}$ followed by centrifugation at $12,000 \mathrm{rpm}$ at $4^{\circ} \mathrm{C}$ for 20 minutes. Supernatant was carefully collected as to not disturb the pellet on the bottom of the test tube and aliquoted into three fresh $1.5 \mathrm{~mL}$ Eppendorf tubes for storage at $-80^{\circ} \mathrm{C}$ until use. Prior to use, protein concentrations were quantified by the Bradford assay (Bio-Rad protein assay dye reagent; Bio-Rad, Hercules, CA) using bovine serum albumin (BSA) as the reference protein for creating a standard curve. Samples were then boiled with $4 x$ loading buffer and $50 \mathrm{mmol} / \mathrm{L}$ dithiothreitol for 5 
minutes at $95^{\circ} \mathrm{C}$ and placed on ice. Nuclear protein was extracted using a Nuclear Extraction Kit (Abcam, ab113474) following manufacturer's instructions for tissue samples.

\section{Western blot analysis}

Protein from the liver tissues were separated on a $10 \%$ sodium dodecylsulfate/polyacrylamide gel electrophoresis (SDS-PAGE) gel at a constant $70 \mathrm{~V}$ until the bromophenol dye was approximately half way down the gel and then increased to a constant $120 \mathrm{~V}$ until the tracking dye reached the bottom of the gel. Protein was transferred to a $0.22 \mu \mathrm{M}$ nitrocellulose membrane (Bio-Rad, Hercules, CA) in transfer buffer containing $20 \mathrm{mM}$ Tris base, $152 \mathrm{mM}$ glycine and $20 \%$ methanol for 90 minutes in a $4{ }^{\circ} \mathrm{C}$ walk-in refrigerator at a constant $250 \mathrm{~mA}$. At the end of the transfer membranes were stained with Ponceau S (Sigma-Aldrich, St. Louis, MO) to reversibly detect protein bands followed by washing $3 x$ for 5 minutes with tris buffered saline contain 10\% tween-20 (TBST) (VWR, Radnor, PA). Membranes were incubated in blocking buffer ( $5 \%$ milk and $0.5 \%$ BSA in TBST) at room temperature for 1 hour, rinsed $2 x$ for 5 minutes with TBST and incubated in primary antibodies diluted in 5\% BSA in TBST against SREBP-1 (Abcam 28481; 1:1000) and GAPDH (Santa Cruz sc-32233; 1:3000) overnight at 4ํㅡ. Membranes were washed $3 x$ for 5 minutes with TBST and incubated with secondary horseradish-peroxidase conjugated antibodies (Anti-mouse IgG 7076 or Antirabbit IgG 7074; Cell Signaling Technology, Danvers, MA) diluted 1:3000 in blocking buffer for 1 hour at room temperature. Membranes were visualized using 
enhanced chemiluminescence $(E C L)$ reagents and a ChemiDoc ${ }^{\mathrm{TM}}$ Touch Imaging System (Bio-Rad, Hercules, CA). The amount of protein was analyzed and normalized against their respective loading controls using Image Lab analysis software version 5.2.1 (Bio-Rad, Hercules, CA).

\section{Western blot analysis for metallothionein}

MT is a small molecular weight ( $6 \mathrm{kDa})$, cysteine rich protein that requires alternate western blot conditions compared to the general protocol described above to ensure the metallothionein protein 1) does not form disulfide bonds with itself or other cysteine rich proteins and 2) successfully transfers from the gel to the membrane and is retained on the membrane for analysis. The transfer conditions for MT are not appropriate for the detection of $\beta$-actin; therefore, 2 parallel gels are run under the same conditions except the gel for MT is transferred to a nitrocellulose membrane in a $15 \%$ methanol, calcium chloride containing buffer while the $\beta$-actin gel, run in parallel, was transferred in a $20 \%$ methanol buffer without calcium chloride. MT protein analysis was calculated as the fold difference relative to control (in the same membrane) with the $\beta$-actin membrane serving only to confirm equal loading of the protein.

For each sample, dithiothreitol was added to the protein/lysis buffer mix to a final concentration of $20 \mathrm{mM}$ and incubated at $56{ }^{\circ} \mathrm{C}$ for 30 minutes followed by the addition of iodoacetamide (IAA) (Sigma-Aldrich, St. Louis, MO) to a final concentration of $50 \mathrm{mM}$ then incubated in the dark at room temperature for 1 hour. IAA was added to covalently bind to the thiol groups of the cysteine residues on 
MT to hinder the formation of disulfide binds. Samples were centrifuged for 5 minutes at $800 \mathrm{rpm}$ and supernatant collected, making sure not to disturb the pellet at the bottom of the tube. Loading buffer ( $9 \mu \mathrm{L} /$ sample) was added, samples vortexed and boiled for 5 minutes at $95^{\circ} \mathrm{C}$ and placed on ice. Samples were subjected to electrophoresis on a $16 \%$ SDS-PAGE gel at a constant $100 \mathrm{~V}$ until the tracking dye reached the bottom of the gel. Gels were incubated in freshly made, ice cold transfer buffer containing $20 \mathrm{mM}$ Tris base, $152 \mathrm{mM}$ glycine, 15\% methanol and $2 \mathrm{mM}$ calcium chloride, dihydrate for 15 minutes before transfer to a $0.22 \mu \mathrm{M}$ nitrocellulose membrane (Bio-Rad, Hercules, CA) at a constant $40 \mathrm{~V}$ for 90 minutes.

After the transfer, membranes were incubated with $2.5 \%$ glutaraldehyde (Fisher Scientific, Waltham, MA) for 1 hour at room temperature to cross-link MT onto the membrane, increasing its retention. Membranes were washed $2 x$ for 5 minutes in phosphate buffered saline (PBS) followed by a 5 minute wash in $50 \mathrm{mM}$ ethanolamine (Sigma-Aldrich, St. Louis, MO) in PBS to quench any residual glutaraldehyde. Membranes were blocked in freshly made 3\% BSA in PBS for 90 minutes at room temperature followed by incubation with primary MT monoclonal antibody (Dako M0639, clone E-9, Santa Clara, CA) diluted 1:1000 in 3\% BSA in PBS overnight at $4^{\circ} \mathrm{C}$.

The next day, membranes were washed $3 x$ for 5 minutes with TBST and incubated with secondary horseradish-peroxidase conjugated antibody (Antimouse IgG 7076; Cell Signaling Technology, Danvers, MA) diluted 1:3000 in 3\% 
BSA in PBS for 1 hour at room temperature. Membranes were visualized as described above.

\section{Metals analysis}

Each whole blood and liver sample (20-100 mg wet-weight) was digested in $1 \mathrm{~mL}$ of $70 \%$ concentrated trace metal grade nitric acid in an $85^{\circ} \mathrm{C}$ water bath for 4 hours. After digestion, samples were cooled to room temperature and filtered using a $100 \mu \mathrm{m}$ filter. Next, $34 \mathrm{~mL}$ of Milli-Q deionized water was added to each sample bringing the final concentration of nitric acid to $2 \%$. Metal levels were assessed using an X Series II quadrupole inductively coupled plasma mass

spectrometry (ICP-MS) (Thermo Fisher Scientific) equipped with an ESI SC-2 autosampler (Elemental Scientific, Inc.) for sample injection. During sample injection, internal standards including $\mathrm{Bi}, \mathrm{In}, \mathrm{Li}, \mathrm{Sc}, \mathrm{Tb}$ and $\mathrm{Y}$ (Inorganic Ventures) were mixed with each sample for drift correction and accuracy improvement. Each sample was analyzed three times and metal levels calculated and presented as $\mathrm{ng} / \mathrm{g}$ wet tissue. Anything less than the Intercept Concentration was considered non-detectable.

\section{Statistical analysis}

Statistical analysis was performed using GraphPad Prism version 7 statistical software (GraphPad Software Inc., San Diego, CA). Results are reported as the mean \pm standard deviation (SD; $\mathrm{n}=2-12$ ). Following two-way ANOVA analysis to determine if there was significant interaction between diet and cadmium 
exposure, Bonferroni's post hoc test was performed using a significance level of $p$ $<0.05$. For Aim 1 and 3: ${ }^{*}, p<0.05$ compared to group control; \#, $p<0.05$ compared to cadmium dose within diet group; @, $p<0.05$ compared to corresponding normal diet. For Aim 2: ${ }^{*}, \mathrm{p}<0.05$ compared to group control; \#, $\mathrm{p}$ $<0.05$ compared to corresponding normal zinc diet; @, $p<0.05$ compared to corresponding normal diet.

For body weight, repeated measures analysis of variance (rANOVA), a special case of linear mixed effect models, was used. In the rANOVA, the main effects (Cd exposure, diet, Zn supplement, time in weeks) and their interactions as fixed effects, and subject as random effects were included. The estimated fixed effects, their standard errors, and p-values were used for statistical inferences. 
CHAPTER 3: RESULTS

\section{Aim 1: Measure the impact of whole-life, chronic cadmium exposure on high}

\section{fat diet-induced NAFLD}

\section{Background}

NAFLD is the most common cause of chronic liver diseases affecting more than $25 \%$ of the world's population (Younossi et al., 2018) and in the United States alone, between 75 and 100 million individuals are afflicted with the disease (Rinella, 2015). Obesity, a major public health concern affecting over 312 million people worldwide, is one of the greatest risk factors for the development of NAFLD (Fakhouri et al., 2013; Ogden et al., 2012; Ruhl and Everhart, 2015). In fact, NAFLD is prevalent in over $90 \%$ of obese patients (Younossi et al., 2018). Interestingly, up to $20 \%$ of NAFLD patients are considered to be of normal weight; therefore indicating the influence of other risk factors in disease development such as genetics and/or environmental exposures.

Even with advances in the field, the knowledge of the mechanism underlying the development and pathogenesis of NAFLD remain incomplete. For example, despite the increasing prevalence of NAFLD, most patients only present with simple steatosis that never progresses to necroinflammation or subsequent fibrosis and chronic liver disease (Buzzetti et al., 2016). Thus, suggesting that addition to a first "hit" that induces steatosis (such as over consumption of a high 
fat diet) the progression of the diseases requires a second "hit" (such as an environmental exposure), a hypothesis proposed by Day and James (2015) (Nakamura and Teraichi, 2013).

Environmental exposure to the non-essential metal cadmium is implicated in the development of several metabolic diseases, including NAFLD (Hyder et al., 2013; Fatima et al., 2019; Little et al., 2020). The liver is a major target organ of cadmium accumulation and although it is clear that chronic cadmium exposure induces hepatotoxicity the mechanism(s) are still not fully understood (Järup et al., 1998; Järup and Akesson, 2009). Limited studies correlating cadmium exposure with metabolic diseases, such as NAFLD, do not take into account such environmental exposures can be life-long and multigenerational nor do they take into account the risk of developing adulthood diseases is correlated with adverse stimuli in utero, resulting in permanent physiological and metabolic changes. Additionally, the impact of whole life exposures to cadmium alone, or in conjunction with high fat diet, on NAFLD have not been investigated. Therefore, we developed an in vivo multiple-hit model to study the effect of whole life, low dose cadmium exposure on high fat diet-induced NAFLD (Figure 3.1).

We adopted this multiple-hit model using a high fat diet to induce steatosis in C57BL/6 mice exposed to cadmium starting in utero, and continuing through adulthood. The C57BL/6 mouse strain is the most widely used nutritional model of NAFLD (Aydos et al., 2019). NAFLD induction by a high fat diet in C57BL/6 mice results in phenotypes similar to those observed in humans with NAFLD (i.e. obesity, 


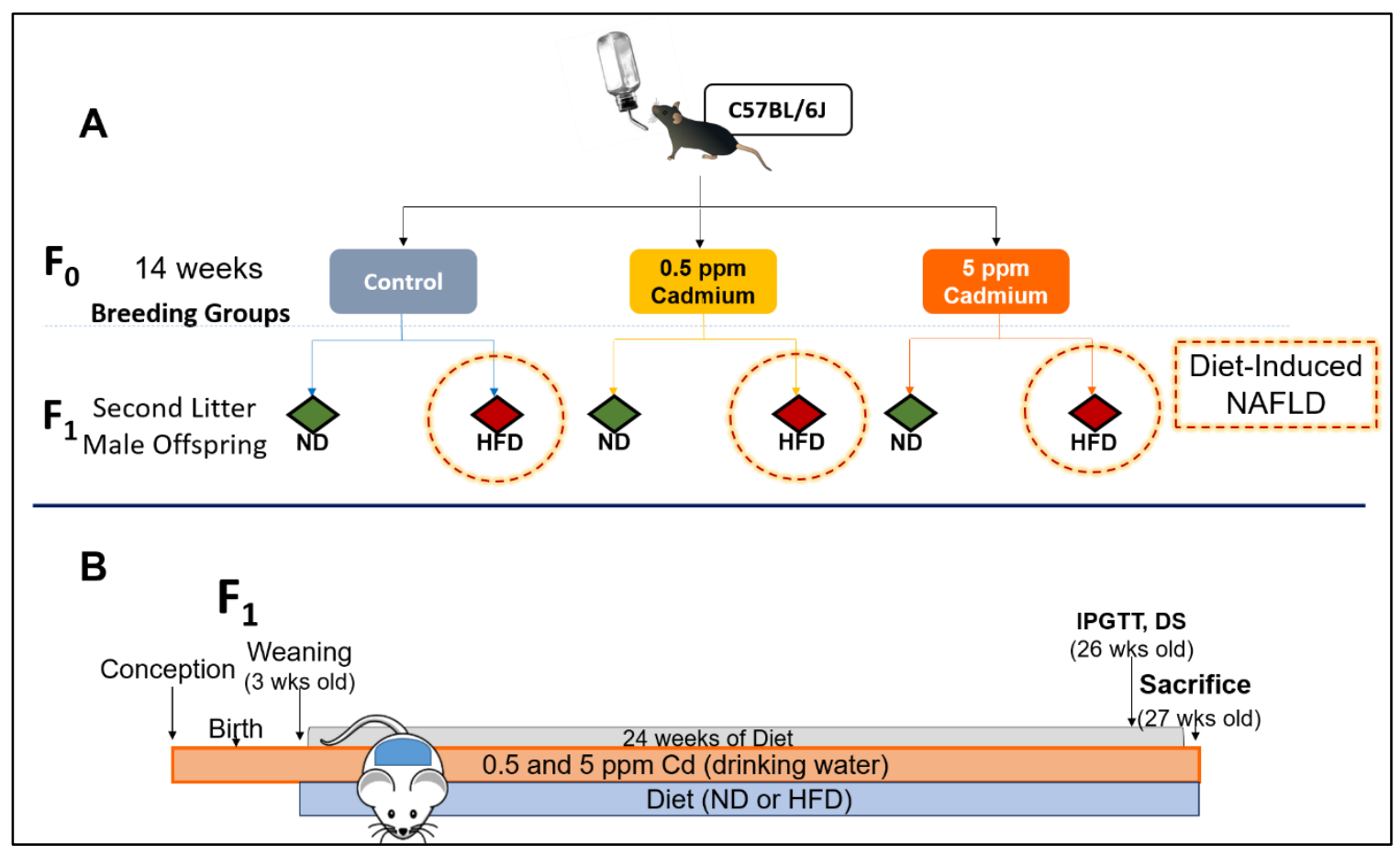

Figure 3.1. Model of whole life exposure of male mice to cadmium and high

fat diet. (A) Adult male and female C57BL/6J mice on defined diets were exposed to control drinking water, or water containing 0.5 or $5 \mathrm{ppm}$ cadmium for 14 weeks before being established into breeding pairs. Pregnant dams and male offspring were continuously exposed and continued on the same drinking water regime as their parents after weaning. At weaning, male offspring were also fed either a normal or high-fat diet (ND or HFD, respectively) for 24 weeks. (B) One week prior to sacrifice, IPGTT tests were performed and body composition determined by DEXAscan (DS). IPGTT = intra-peritoneal glucose tolerance test, wks $=$ weeks 
hyperglycemia and liver damage) and reflect the natural course of the disease in humans (Nakamura and Teraichi, 2013; Aydos et al., 2019). Using this model, we asked three primary questions: 1) Did our high fat diet model successfully induce NAFLD?; 2) Did our cadmium exposure model result in hepatic cadmium accumulation?; and 3). Did whole life exposure to low concentrations of cadmium exacerbate high fat diet-induced NAFLD?

\section{Findings}

\section{Characterization of the high fat diet model used to induce NAFLD}

We first wanted to confirm our high fat diet model was successful. It is well known that diets high in fat promote weight gain and obesity (Golay and Bobbioni, 1997). Although BMI is used as the leading measure of obesity, a number of studies have shown this measurement has limited ability to accurately determine body composition (Kennedy et al., 2009; Heymsfield and Cefalu, 2013; Ponti et al., 2019). DEXAscan is considered a much more accurate measure of obesity, providing fat, bone and lean tissue measurements (Shepherd et al., 2017). Additionally, diets high in fat are a major risk for insulin resistance (Park et al., 2001; Winzell et al., 2004) and increased blood sugar levels in combination with increased insulin in the blood are indicators of insulin resistance (Bowe et al., 2014). Furthermore, insulin resistance is a hallmark of NAFLD that greatly influences disease progression (Manco, 2017). Therefore, we evaluated our model with these four measures: Body weight, DEXAscan, IPGTT and plasma insulin. 
We tracked body weight gain over time and assessed body mass composition with DEXAscan technology and found the expected outcomes; mice fed high fat diet gained significantly more weight overtime $(p=<0.001)$ (Figure 3.2A) and had greater percent fat and lean tissue mass compared to normal diet fed mice (Figure 3.2B and E). The DEXAscan further showed consumption of high fat diet did not impact bone mineral density or bone mineral content (Figure 3.2F and G). Further indicative of a successful model, the diet high in fat significantly increased blood glucose levels compared to normal diet fed animals (Figure 3.3A and B) with a trend towards increased insulin in the plasma (Figure $3.3 \mathrm{C}$ ) suggesting the development of diet-induced insulin resistance

Next, we assessed the ability of our high fat diet model to induce NAFLD. The ratio of liver weight at sacrifice to the length of the tibia is an indicator of hepatomegaly (enlarged liver) and suggests hepatic damage. Elevated ALT and AST, transaminases released into the bloodstream upon injury to the liver, are widely used laboratory indicators of hepatocellular damage. Histologically, liver injury can be further assessed by examination of hematoxylin and eosin (H\&E) stained paraffin embedded tissue for overall hepatic structure and oil red $O$ staining to determine the presence of fat, which is commonly confirmed with biochemical analysis of hepatic triglyceride levels. Therefore, we evaluated NAFLD in our model with the following five measures: liver to tibia ratios, plasma ALT and AST, H\&E, oil red $\mathrm{O}$ and hepatic triglycerides.

Mice fed high fat diet tended to have enlarged livers (Figure 3.4A) and increased ALT, but not AST, levels compared to normal diet fed mice (Figure 3.4B 

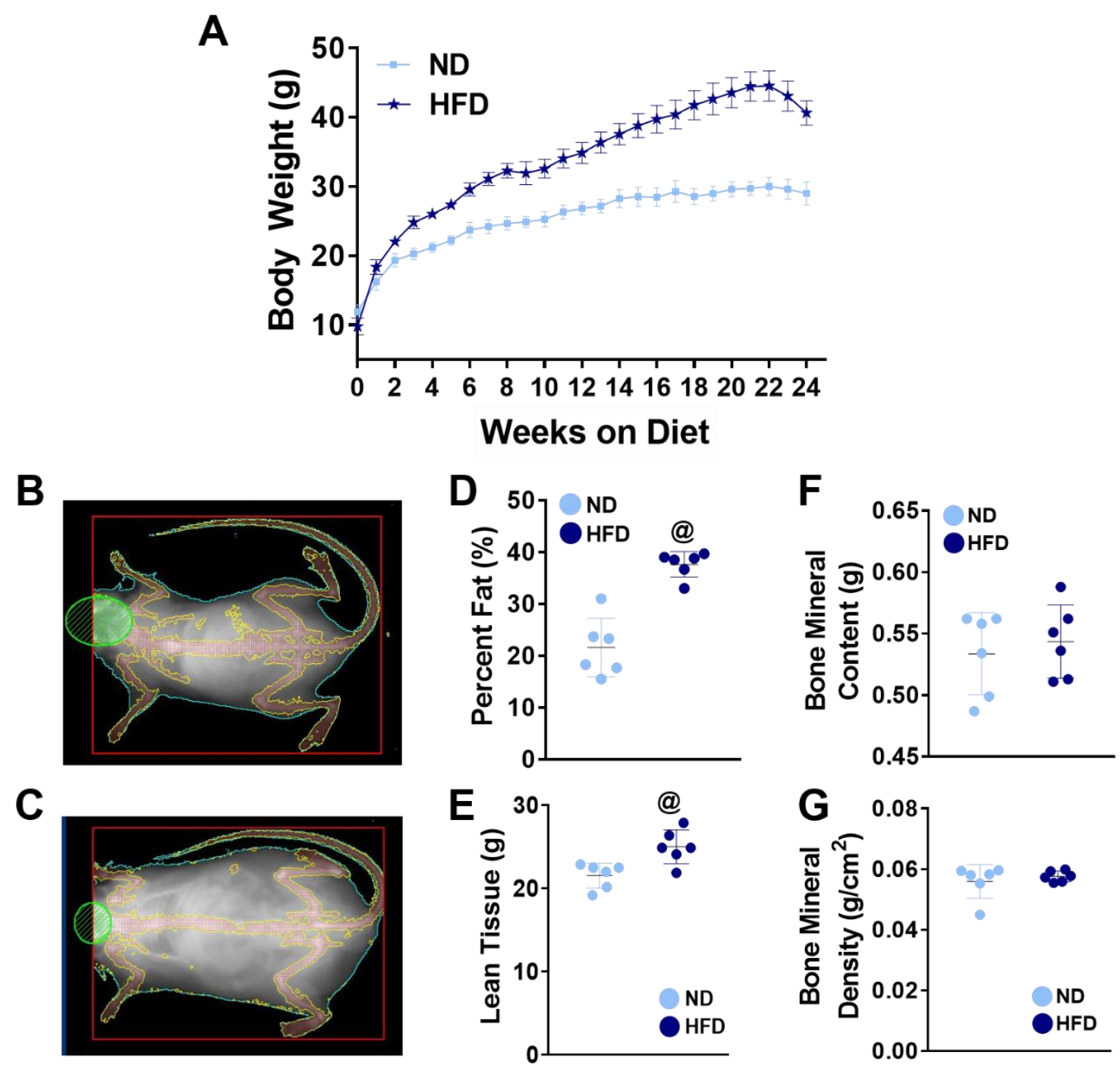

Figure 3.2. Effects of high fat diet on body weight and composition in male mice. This figure shows consumption of high fat diet for 24 weeks post weaning (A) increased body weight gain over time and (B: representative DEXAscan photo of a normal diet fed mouse; C: representative DEXAscan photo of a high fat diet fed mouse) changed body composition, increasing (D) percent fat and (E) lean tissue mass, but not $(F)$ bone mineral content or $(G)$ bone mineral density. Results are reported as the mean \pm SD for DEXAscan $(n=6)$ and mean \pm SEM $(n=6)$ for body weights. @, p $<0.05$ compared to normal diet. 


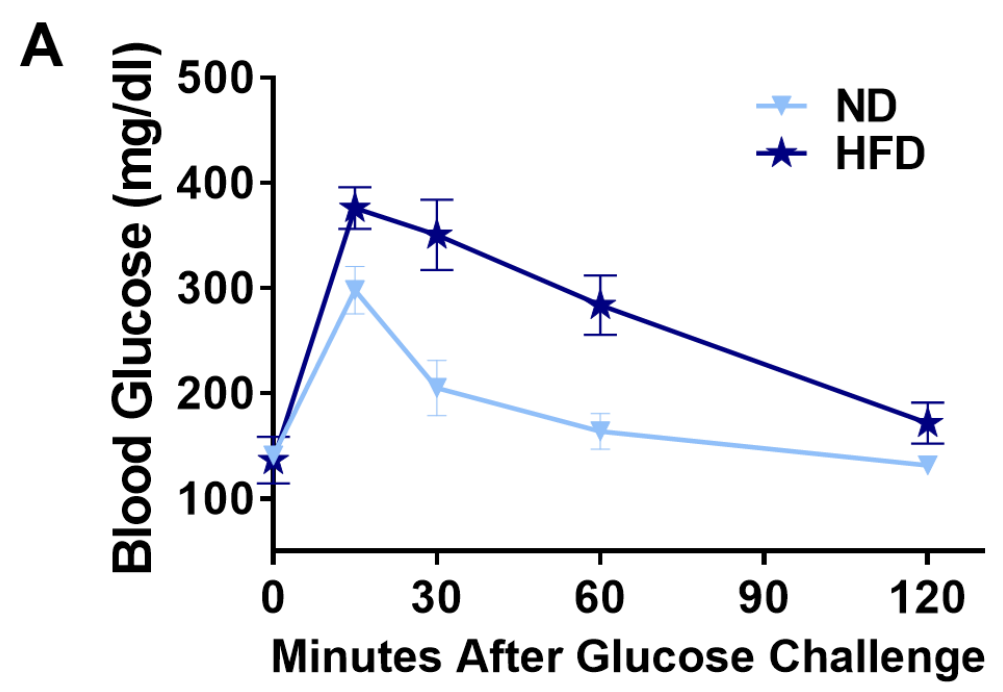

B

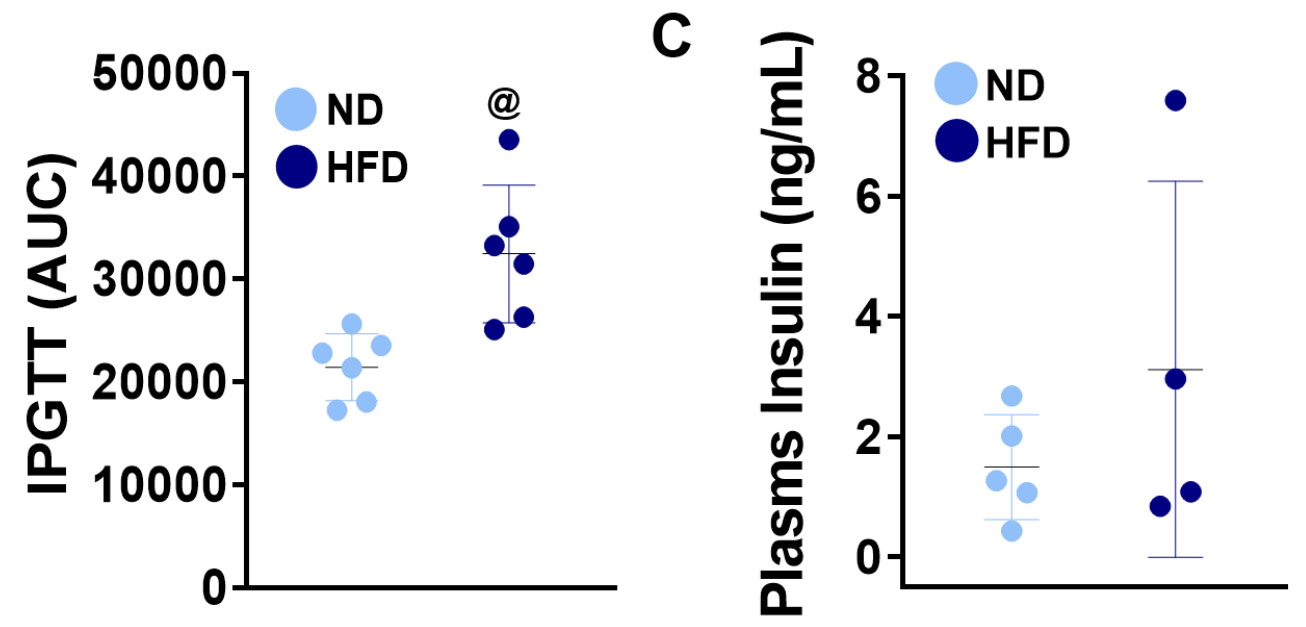

Figure 3.3. Effects of high fat diet on glucose handling and plasma insulin in male mice. This figure shows consumption of high fat diet for 24 weeks post weaning (A) significantly decreased glucose tolerance (indicated by the area under the curve (AUC) from IPGTT), (B) increased blood glucose levels and (C) tended to increase plasma insulin levels. Taken together, these result are indicative of insulin resistance. Results are reported as the mean \pm SD $(n=6)$. @, $p<0.05$ compared to normal diet. 
A

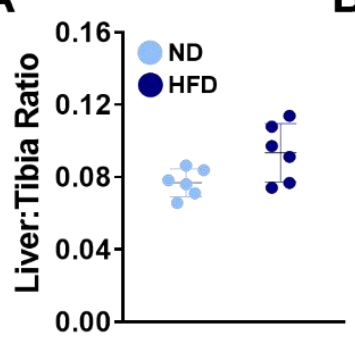

D
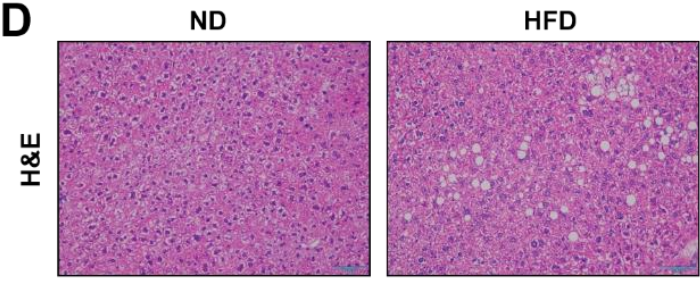

B

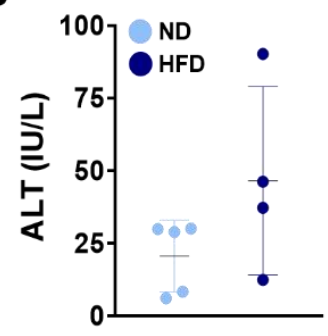

C

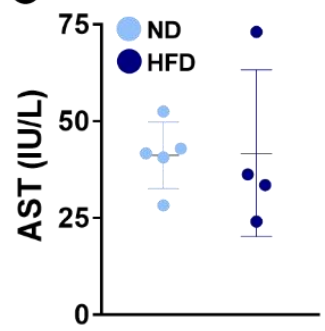

E

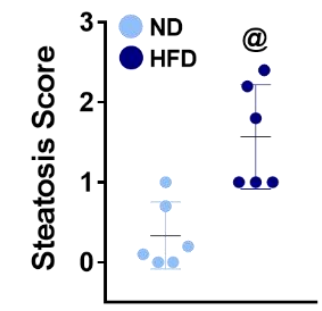

$\mathbf{F}$

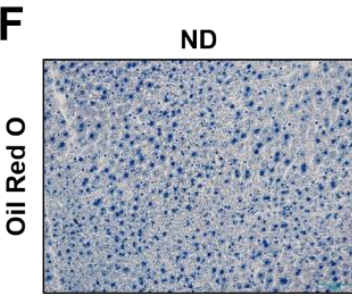

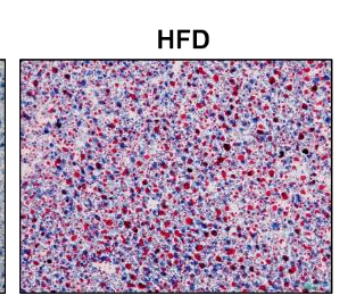
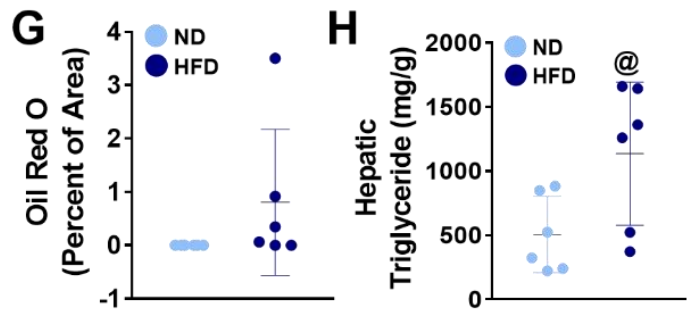

Figure 3.4. Effects of high fat diet on liver injury and NAFLD in male mice.

This figure shows consumption of high fat diet for 24 weeks causes liver injury as indicated by (A) enlarged livers (hepatomegaly determined by liver:tibia ratio), (B) increased plasma ALT, but (C) not AST, and (D-H) steatosis. (D) Representative photomicrographs of paraffin embedded liver tissue stained with hematoxylin \& eosin staining (H\&E, 200x). (E) Steatosis was scored as percent of liver cells in 5, 10x fields per liver containing fat. $(F)$ Representative photomicrographs of ORO (neutral lipids, $\times 200$ ) stained frozen liver sections. (G) Image analysis of OROpositive staining was performed using Image $\mathrm{J}$, and results are shown as percentage of microscope field. $(\mathrm{H})$ Biochemical quantification of hepatic triglycerides. Results are reported as the mean $\pm S D(n=4-6)$. @, $p<0.05$ compared to normal diet. 
and C), although not statistically significant. Histological analysis of liver tissue revealed significant increases in steatosis and lipid deposition in mice fed high fat diet compared to controls as seen in representative photomicrographs of $\mathrm{H} \& \mathrm{E}$ (Figure 3.4D) and oil red $\mathrm{O}$ stained liver tissue (Figure 3.4F) as well as in the quantification of the histology (Figure 3.4E and G). Additionally, biochemical analysis of liver tissue showed high fat diet fed mice had greater hepatic triglyceride levels compared to mice fed normal diet (Figure 3.4H). These NAFLD markers combined with the changes in weight, percent fat, lean tissue mass, blood glucose and plasma insulin show that our model of high fat diet-induced NAFLD was successful.

\section{Characterization of the cadmium exposure model}

We also wanted to confirm that our cadmium exposure model was successful. The liver is a major target organ of cadmium toxicity and accumulation with a hepatic half-life of between 4-19 years (ATSDR, 1999; Hyder et al., 2013). Metallothionein, a small, low molecular weight, cysteine rich protein, plays a major role in protecting the body from cadmium toxicity and the expression of metallothioneins generally increases with elevations in tissue cadmium levels (Klaassen et al., 2009). There are 4 major mammalian isoforms of metallothionein, of which MT-1 and MT-2 are highly expressed in the liver (Klaassen et al., 1999). Therefore, we evaluated our cadmium exposure model by measuring cadmium levels in liver tissue using ICP-MS and determining metallothionein protein and mRNA (both MT-1 and MT-2) levels in the liver. 
As expected, cadmium accumulated in the liver in a concentrationdependent manner in both normal and high fat diet fed mice (Figure 3.5A). High fat diet consumption did not influence cadmium accumulation in control or $5 \mathrm{ppm}$ exposed mice; however, high fat diet fed mice exposed to the lower concentration of cadmium $(0.5 \mathrm{ppm})$ had greater hepatic cadmium content compared to the corresponding normal diet fed mice, although not statistically significant.

Additionally, in our model, mice were exposed to cadmium in their drinking water, therefore we measured water consumption and calculated the average administered cadmium intake (Figure 3.5B and C). Over the course of the study, 0.5 and 5 ppm cadmium exposed male mice drank an average of $5.15 \pm 1.45$ and $6.13 \pm 1.45 \mathrm{~mL}$ of water per a day, respectively, compared to controls who drank an average of $4.60 \pm 0.55 \mathrm{~mL}$ of water per a day. Thus, treated mice drank similar amounts of water compared to controls, independent of diet. The average daily dose of cadmium in mice that drank water with $0.5 \mathrm{ppm}$ cadmium was $2.58 \pm 0.62$ and $2.87 \pm 0.71 \mu \mathrm{g}$ in normal and high fat diet fed mice, respectively. In mice that drank water with 5 ppm cadmium the average daily dose was significantly greater: $30.67 \pm 7.27(p=<0.0001)$ and $24.53 \pm 5.42 \mu g(p=<0.0001)$ in normal and high fat diet fed mice, respectively. Interestingly, in 5 ppm cadmium exposed male mice fed high fat diet, cadmium intake was significantly decreased $(p=0.012)$ compared to exposed, normal diet fed mice.

Overall, cadmium accumulated in our target organ, the liver. Therefore, we next focused on determining the response of metallothionein in protection of the liver against cadmium accumulation. In mice fed normal diet, exposure to $5 \mathrm{ppm}$ 

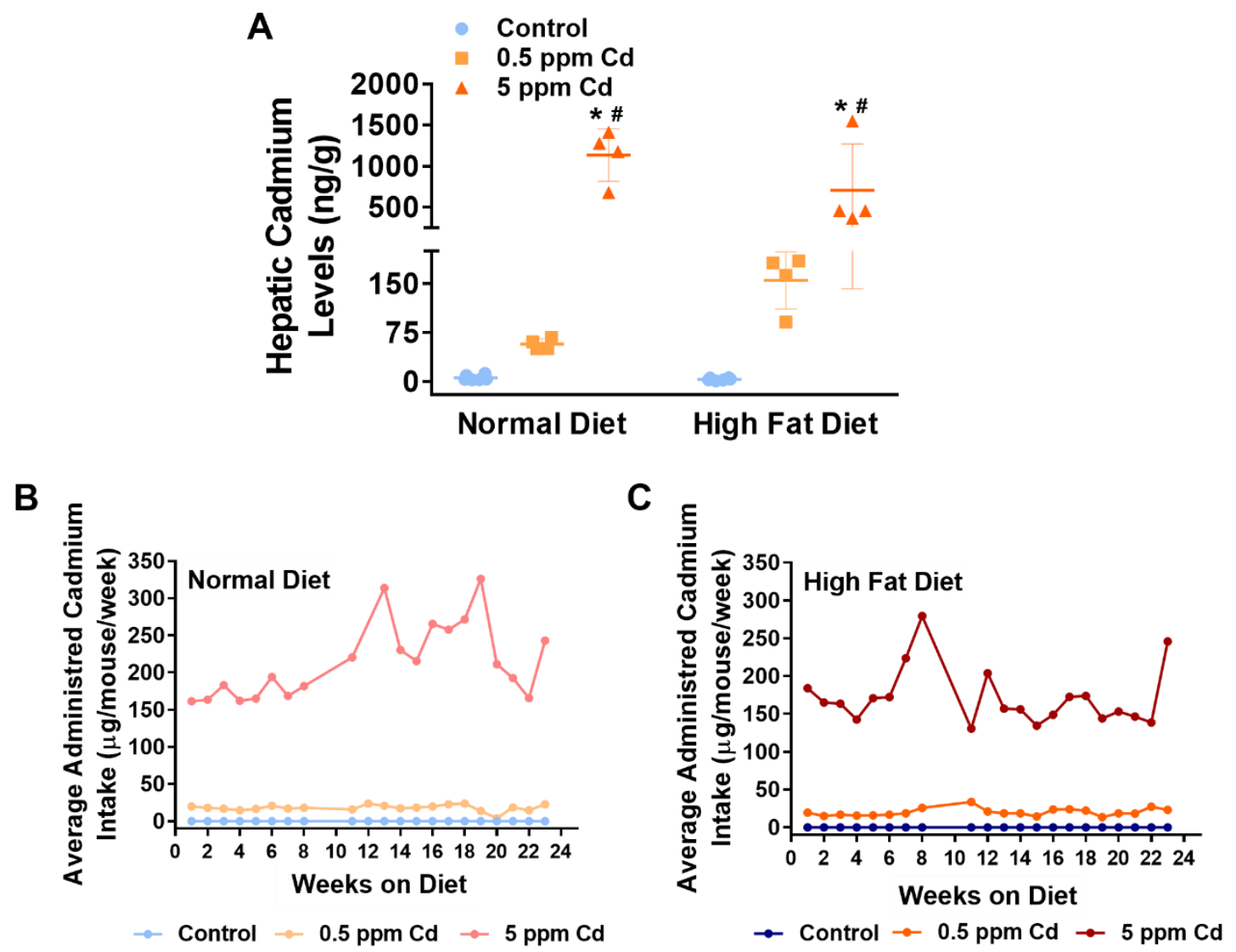

Figure 3.5. Hepatic cadmium levels and weekly administered cadmium intake in male mice. This figure shows cadmium accumulated in the liver in a concentration-dependent manner, reflecting the average administered cadmium intake delivered by drinking water. (A). Hepatic cadmium levels measured by ICPMS. (B) Average weekly administered cadmium intake delivered by drinking water in normal diet fed mice. (C) Average weekly administered cadmium intake delivered by drinking water in high fat diet fed mice. *,$p<0.05$ compared to group control; \#, p < 0.05 compared to cadmium dose within diet group. 
A

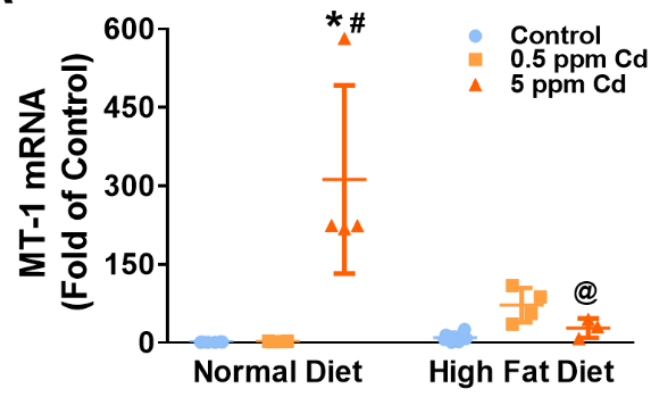

C

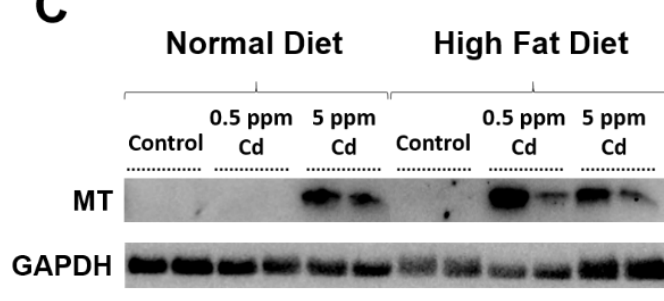

$\mathbf{E}$

Controls

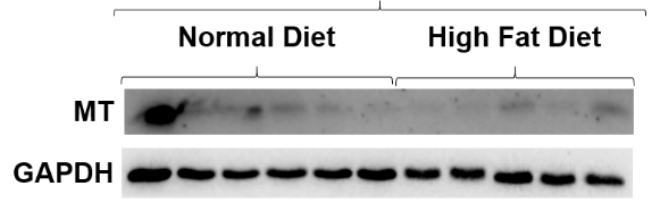

G

$0.5 \mathrm{ppm} \mathrm{Cd} \quad 5 \mathrm{ppm} \mathrm{Cd}$

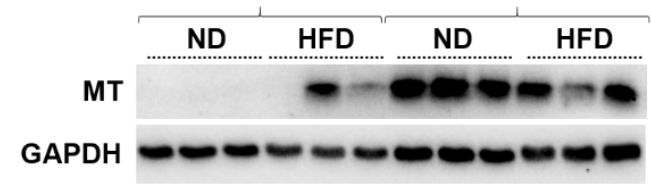

B

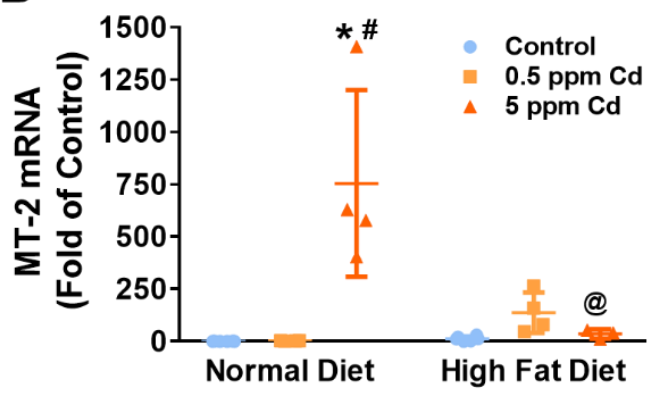

D

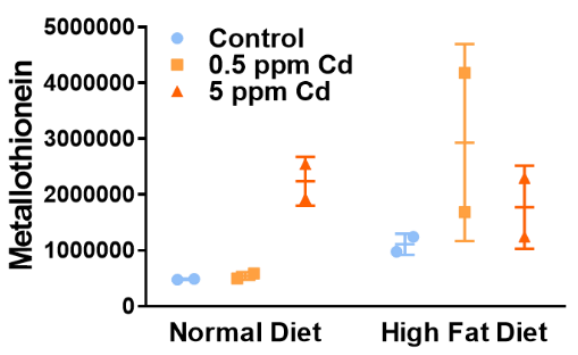

$\mathbf{F}$

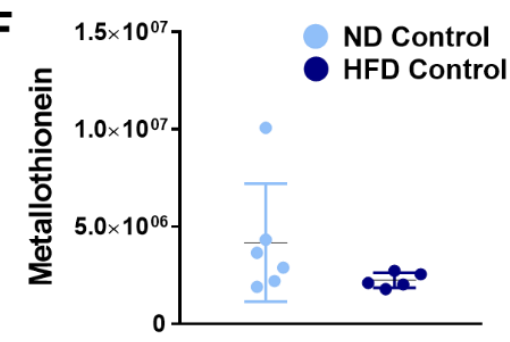

H

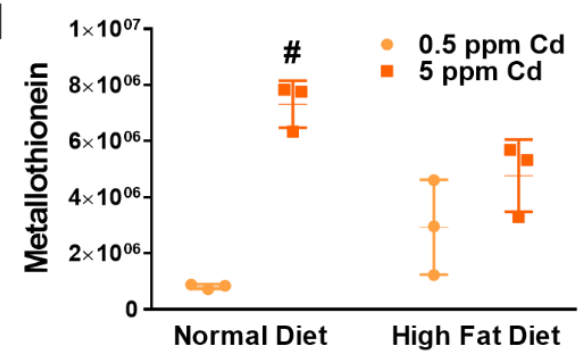

Figure 3.6. Effects of whole life cadmium exposure and high fat diet on metallothionein (MT) in male mice. Cadmium exposure alters MT. (A) Hepatic mRNA expression of MT-1 and (B) MT-2. (C) Representative western blot for hepatic MT in all exposure groups (NOTE: membrane cropped to remove loading dye lane in the middle of the blot) and (D) corresponding densitometric analyses shown as fold of control. (E) Representative western blot for hepatic MT in control 
$\underline{\text { mice }}$ and $(F)$ corresponding densitometric analyses shown as fold of control. (G) Representative western blot for hepatic MT in cadmium exposed mice and $(\mathrm{H})$ corresponding densitometric analyses shown as fold of control. GAPDH was used as a loading control. Results are reported as the mean $\pm S D(n=2-6){ }^{*}, p<0.05$ compared to group control; \#, $\mathrm{p}<0.05$ compared to cadmium dose within diet group; @, $p<0.05$ compared to corresponding normal diet. 
cadmium resulted in a significant increase in mRNA levels of metallothionein compared to controls, a result not observed in $0.5 \mathrm{ppm}$ exposed mice (Figure 3.6A and B). High fat diet alone did not alter hepatic mRNA levels of metallothionein; however, there was a slight increase in metallothionein mRNA levels in high fat diet fed mice exposed to $0.5 \mathrm{ppm}$ cadmium. In contrast, in high fat diet fed mice exposed to $5 \mathrm{ppm}$ cadmium, metallothionein mRNA expression significantly decreased compared to those fed normal diet.

In the liver, metallothionein protein levels showed a similar pattern to that of metallothionein mRNA. Panel C (Figure 3.6) shows a representative western blot containing samples $(n=2)$ for each of the six experimental groups and Panel $D$ (Figure 3.6) shows quantification of the western blot; however, the protein levels were so great in the cadmium exposed groups, that we were unable to detect bands in any of the control mice and we were unable to clearly determine if there were differences in metallothionein between cadmium exposure groups. Therefore, we performed additional western blots to address these questions. Panel E (Figure 3.6) is a representative western blot of control mice in which we were able to observe faint bands in both normal and high fat diet fed animals. Panel F (Figure 3.6) shows the quantification of the western blot. As was seen with mRNA, high fat did not change hepatic metallothionein protein levels.

Panel G (Figure 3.6) shows a representative western blot of cadmium exposed mice and Panel $\mathrm{H}$ (Figure 3.6) shows the quantification. For normal diet animals, exposure to $5 \mathrm{ppm}$ cadmium significantly increased metallothionein protein levels compared to mice exposed to $0.5 \mathrm{ppm}$ cadmium, which was 
consistent with the mRNA level results. By contrast, for high fat diet animals, 0.5 ppm cadmium increased metallothionein protein levels, but 5 ppm cadmium tended to decrease levels such that high fat diet mice had lower metallothionein levels compared to normal diet mice. These outcomes were consistent with the mRNA data.

Thus, our data showed cadmium reaches the liver and metallothionein is affected. Interestingly, the data begin to show different outcomes for $0.5 \mathrm{ppm}$ and $5 \mathrm{ppm}$ cadmium exposures. Exposure to $5 \mathrm{ppm}$ cadmium increased both metallothionein mRNA and protein levels in mice fed normal diet but did not do so in high fat diet fed mice. In contrast, exposure to $0.5 \mathrm{ppm}$ cadmium did not change metallothionein mRNA or protein levels normal diet fed mice, but did tend to increase these measures in high fat diet fed mice.

\section{Impact of cadmium exposure on high fat diet-induced NAFLD}

Once we confirmed we had a working model of high fat diet-induced NAFLD and a successful cadmium exposure model we determined the impact of cadmium exposure on high fat diet-induced NAFLD using the same nine measures implemented in our model confirmation.

\section{Body weight and composition}

Normal-diet-fed mice exposed to $5 \mathrm{ppm}$ cadmium had slightly reduced weight gain compared to control and $0.5 \mathrm{ppm}$ exposed mice, although not statistically significant. (Figure 3.7A). High-fat-diet-fed mice exposed to $5 \mathrm{ppm}$ 
A

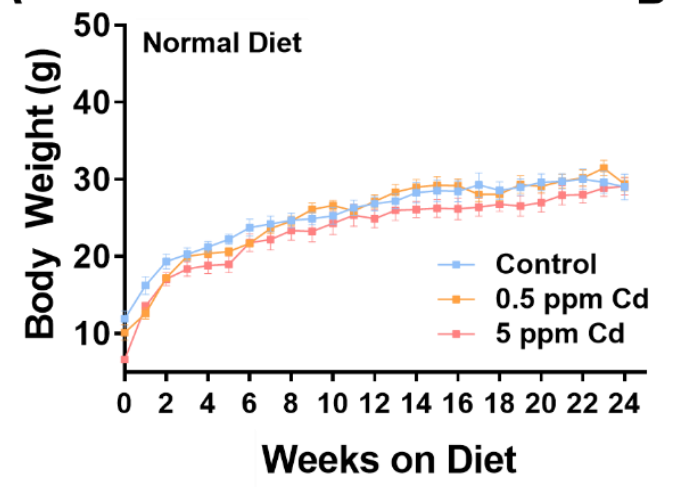

C

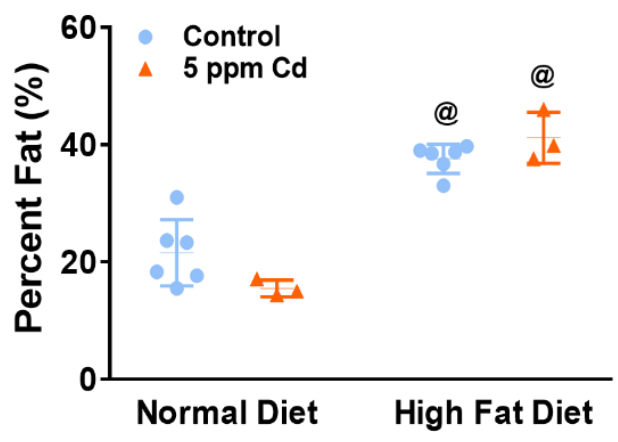

D

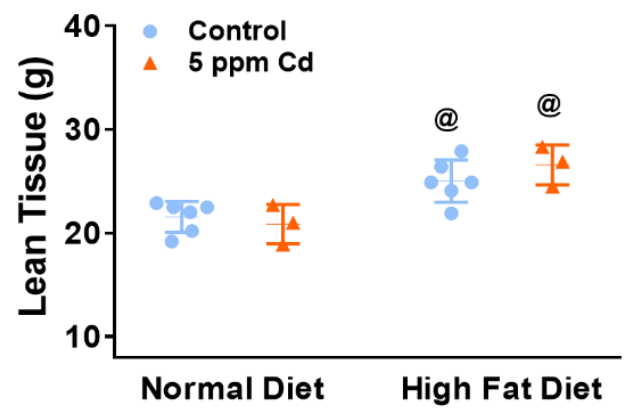

B

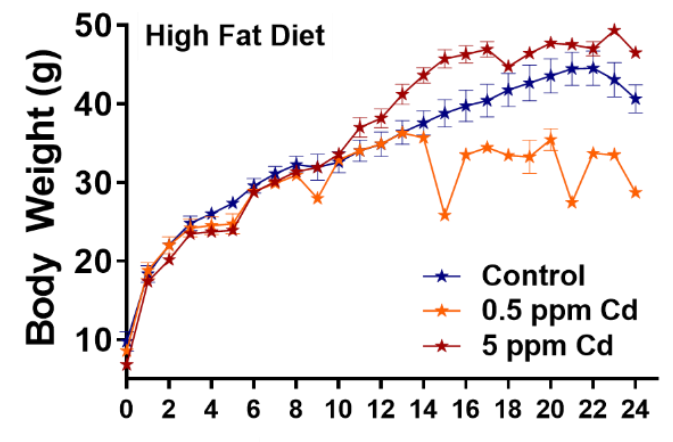

Weeks on Diet

E
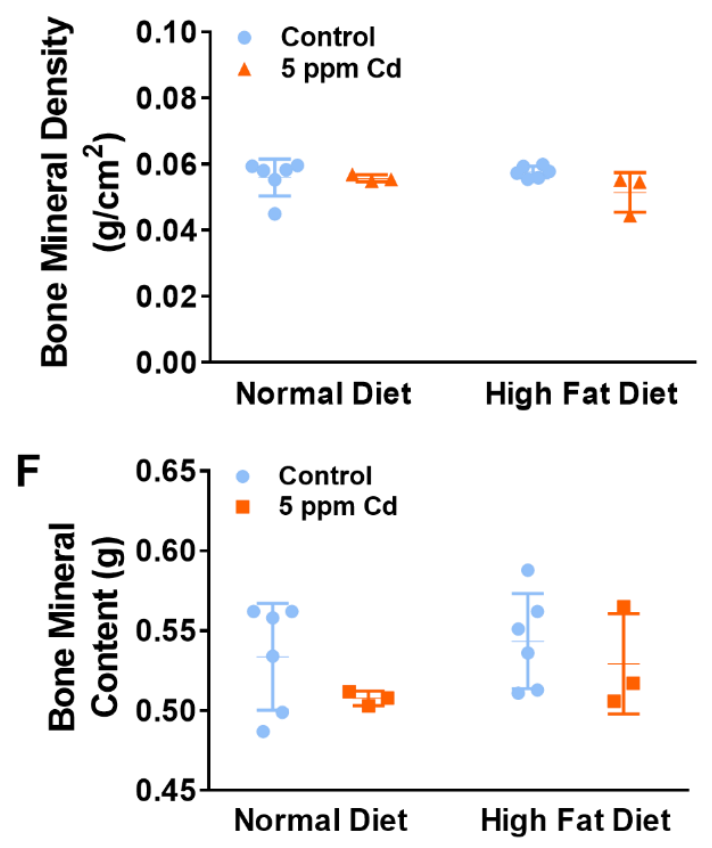

Figure 3.7. Effects of whole life cadmium exposure and high fat diet on body weight and composition in male mice. Whole life exposure to cadmium alters body weight gain over time in high fat diet fed mice, but not body composition. Body weight was measure once a week for 24 weeks starting at weaning, through until sacrifice. Weekly body weight in (A) normal diet fed male mice and (B) high fat diet fed mice. DEXAscan was performed one week prior to sacrifice for control and 5 ppm cadmium exposed mice to assess body composition changes. (C) 
Percent fat, $(D)$ lean tissue mass, $(E)$ bone mineral density and $(F)$ bone mineral content. Results are reported as the mean \pm SD for DEXAscan $(n=3-6)$ and mean \pm SEM ( $n=4-6)$ for body weights. @, $p<0.05$ compared to corresponding normal diet. 
cadmium gained significantly more weight compared to their corresponding high fat diet-fed controls $(p=<0.001)$, while high-fat-diet-fed mice exposed to $0.5 \mathrm{ppm}$ cadmium had reduced weight gain compared to their corresponding controls $(p=$ $<0.001$ ) (Figure 3.7B). These data suggest $5 \mathrm{ppm}$ cadmium exacerbates high fat diet-induced weight gain while $0.5 \mathrm{ppm}$ cadmium reduces weight gain resulting from eating a high fat diet. DEXAscans on control and 5 ppm cadmium exposed mice, did not indicate any further changes in body composition (i.e. \% fat, lean tissue mass, bone mineral density or bone mineral content) as a result of cadmium exposure (Figure 3.7C-F). Mice exposed to $0.5 \mathrm{ppm}$ cadmium were excluded from DEXAscans due to budgetary limitations.

IPGTT and plasma insulin

In normal diet fed mice, cadmium exposure did not significantly alter glucose clearance (Figure 3.8A and C). Similarly, 5 ppm cadmium exposure did not significantly impact high-fat-diet-induced impairment of glucose clearance (Figure 3.8B and C). However, mice exposed to $0.5 \mathrm{ppm}$ cadmium actually showed improved glucose clearance and reduced plasma insulin levels similar to those observed in normal diet-fed animals (Figure 3.8D).

Hepatic injury and NAFLD

High fat diet fed mice exposed to 5 ppm cadmium had significantly larger livers compared to those fed normal diet and compared to high fat diet-fed controls, showing exposure to $5 \mathrm{ppm}$ significantly exacerbated high fat diet-induced hepatomegaly (Figure 3.9A). Interestingly, independent of diet, exposure to $5 \mathrm{ppm}$ 
A

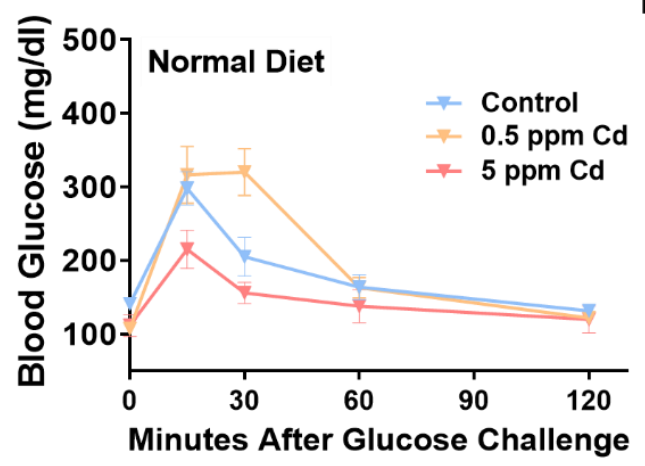

B
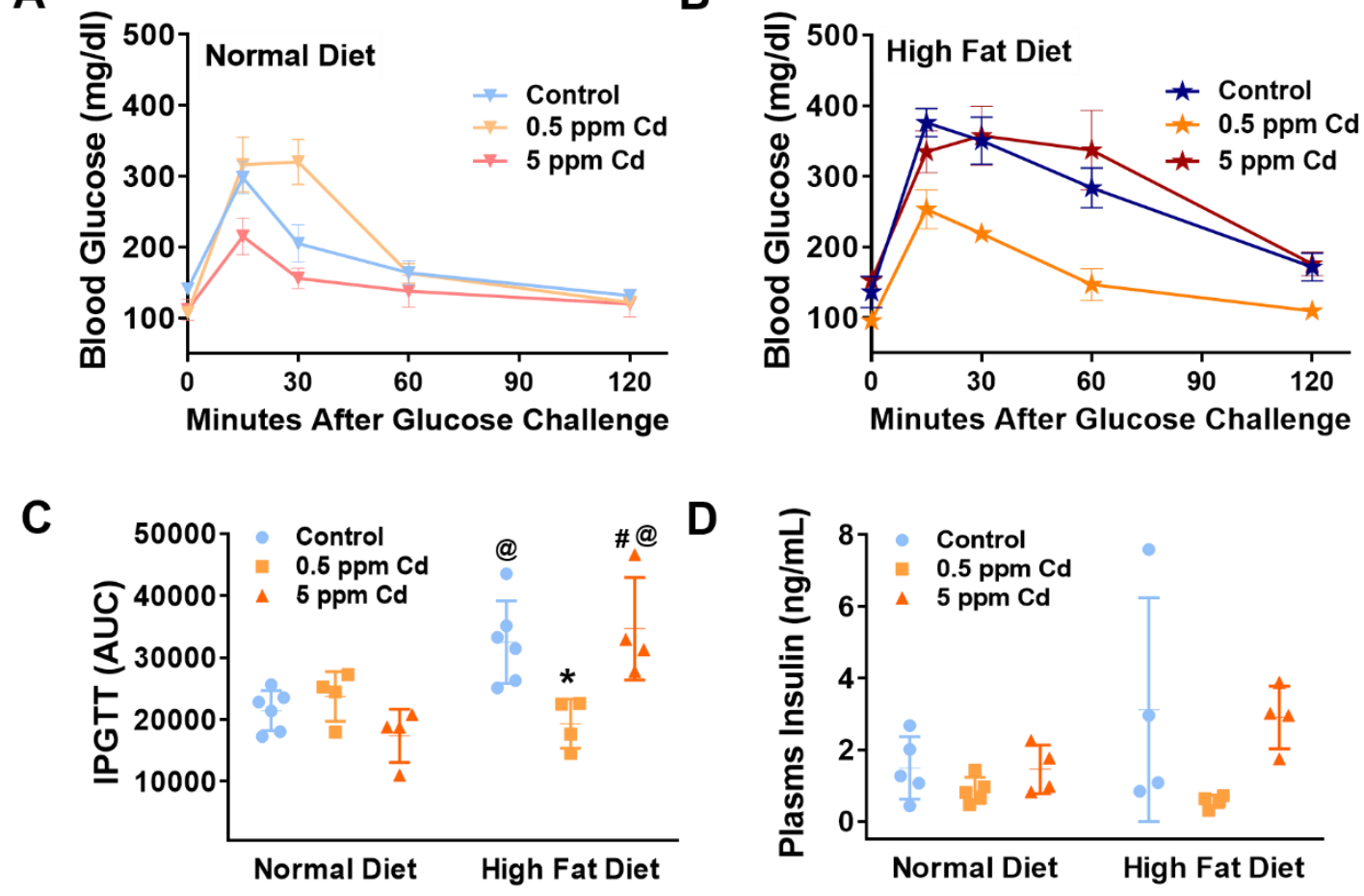

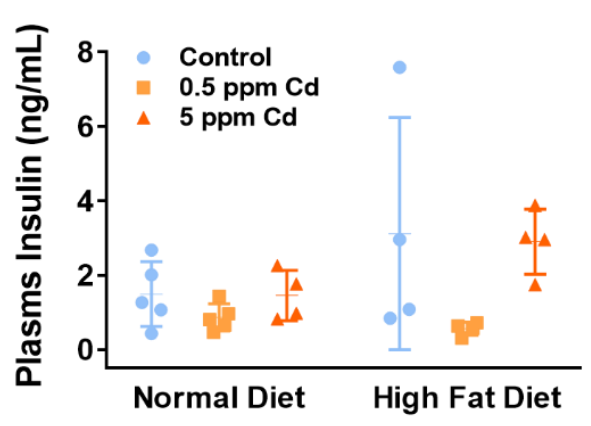

Figure 3.8. Effects of whole life exposure to cadmium and high fat diet on glucose handling and plasma insulin in male mice. Whole life exposure to cadmium alters glucose clearance and plasma insulin in high fat diet fed male mice. Blood glucose levels in (A) normal diet fed mice and (B) high fat diet fed mice after IPGTT, performed one week prior to sacrifice. (C) Integrated area under the curve (AUC) showing quantitative changes in blood glucose levels after glucose challenge. (D) Insulin levels in plasma at time of sacrifice. Results are reported as the mean \pm SD ( $n=4-6) .{ }^{*}, p<0.05$ compared to group control; \#, $p<0.05$ compared to cadmium dose within diet group; @, $\mathrm{p}<0.05$ compared to corresponding normal diet. 
cadmium caused liver injury as indicated by increased ALT and AST levels, suggesting exposure to cadmium alone inflicts damage to the liver (Figure 3.9B and C).

Liver damage was not reflected in normal diet-fed mice according to liver morphology (Figure 3.10A and C) and lipid deposition (Figure 3.10B and D); however cadmium exposure did significantly impact these measures in high fat diet-fed mice. More specifically, 5 ppm cadmium exacerbated high fat diet-induced steatosis and biochemical analysis of hepatic triglyceride content confirmed these results (Figure 3.11A). However, total hepatic cholesterol levels remained unchanged (Figure 3.11B).

Similar to $5 \mathrm{ppm}$ cadmium, exposure to $0.5 \mathrm{ppm}$ cadmium did not overtly impact liver morphology or lipid deposition in normal diet fed mice. However, the outcomes in high fat diet mice after $0.5 \mathrm{ppm}$ cadmium continued to be very different from $5 \mathrm{ppm}$ cadmium. In fact, $0.5 \mathrm{ppm}$ cadmium reduced the size of the high fat diet-enlarged livers, down to levels seen in normal diet fed mice and did not result in liver injury (Figures 3.9A-C). Consistent with these observations, $0.5 \mathrm{ppm}$ cadmium reduced the pathology to levels seen in normal diet fed mice (Figure 3.10 A-D). Taken together these data indicate exposure to $5 \mathrm{ppm}$ cadmium exacerbates high fat diet-induced NAFLD while exposure to $0.5 \mathrm{ppm}$ cadmium attenuates the diet-induced hepatic pathology, possibly through altering hepatic lipid synthesis.

We also delved deeper into a possible underlying molecular mechanism. Dysregulation of hepatic lipid homeostasis is a hallmark of NAFLD (Moslehi and Hamidi-Zad, 2018). The master regulator of hepatic lipid synthesis, sterol 


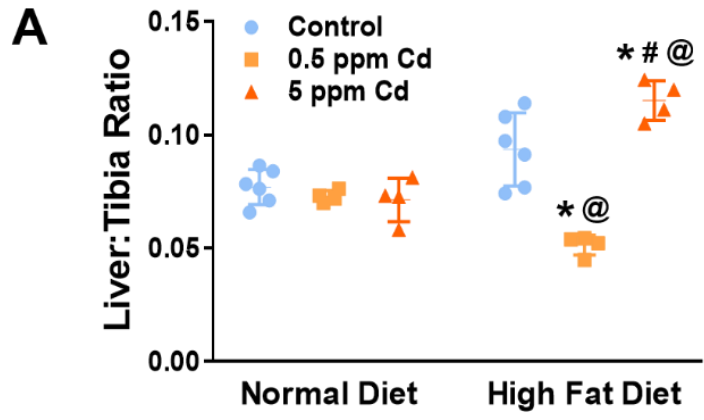

B

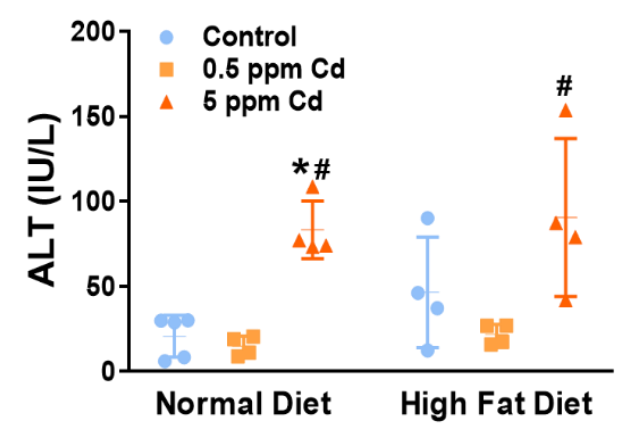

C

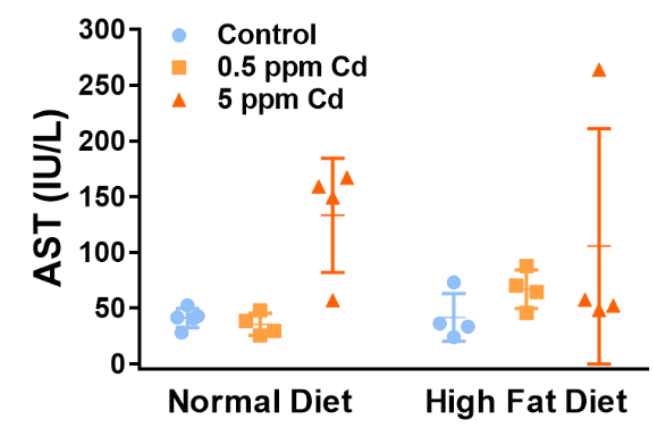

Figure 3.9. Effects of whole life cadmium exposure and high fat diet on liver injury in male mice. This figure shows cadmium exposure changes liver size in high fat diet fed mice and exposure to $5 \mathrm{ppm}$ cadmium increases liver transaminases, independent of diet. (A) Ratio of liver weight in grams to tibia length in millimeters, a measure of hepatomegaly. (B) Plasma aspartate aminotransferase (AST) and (C) plasma alanine aminotransferase (ALT) activity. Results are reported as the mean \pm SD $(n=4-6) .{ }^{*}, p<0.05$ compared to group control; \#, p $<0.05$ compared to cadmium dose within diet group; @, $p<0.05$ compared to corresponding normal diet. 
A
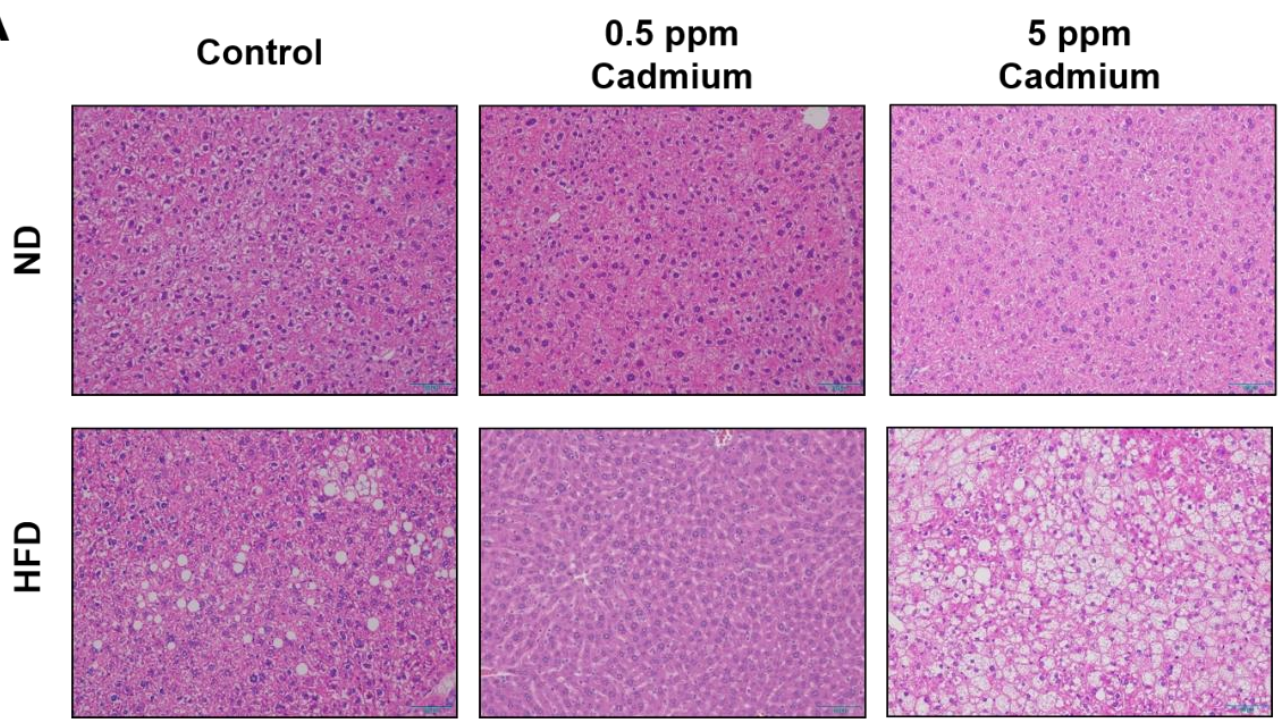

B
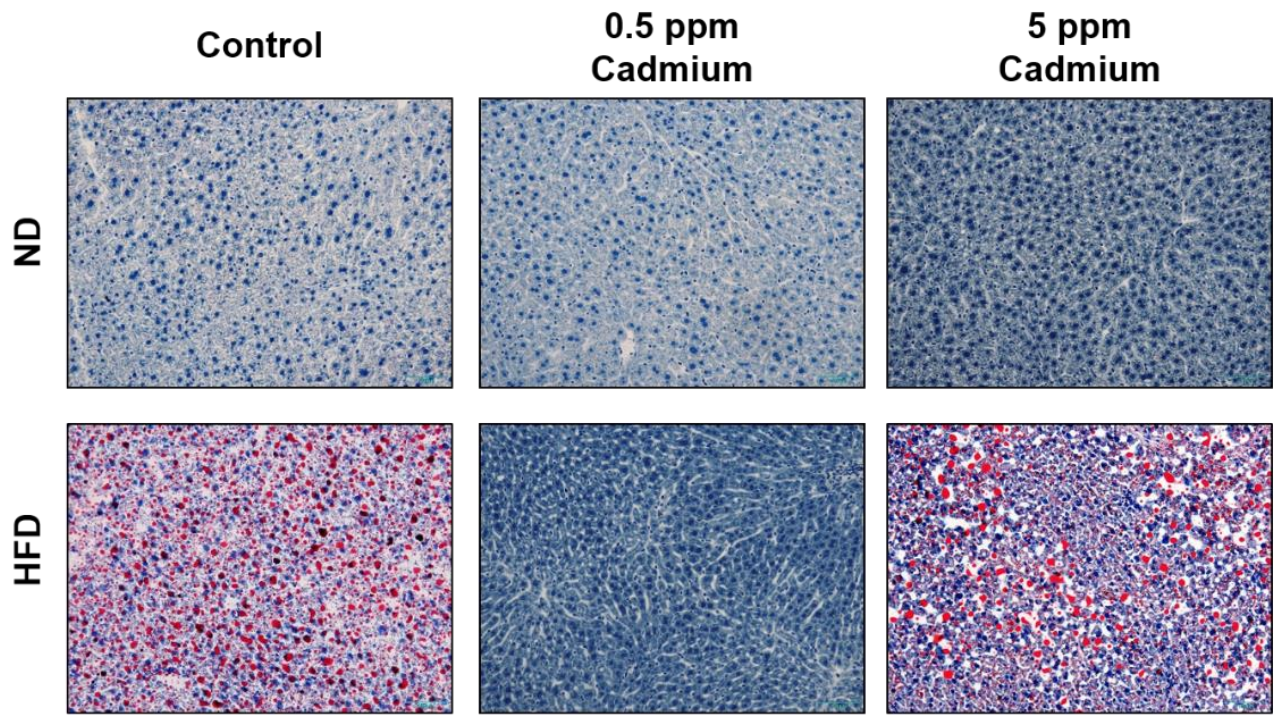

C
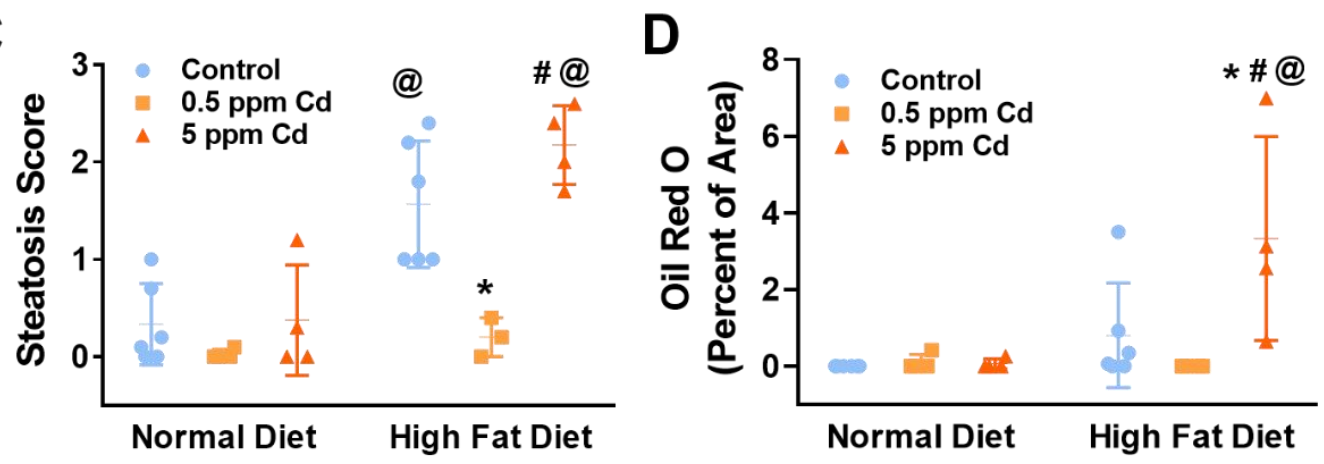

Figure 3.10. Effects of whole life cadmium exposure and high fat diet on NAFLD in male mice. This figure shows exposure to $5 \mathrm{ppm}$ cadmium exacerbated 
high fat diet-induced steatosis while exposure to $0.5 \mathrm{ppm}$ cadmium rescues high fat diet-induced steatosis. (A) Representative photomicrographs of paraffin embedded liver tissue stained with hematoxylin \& eosin staining (H\&E, 200x). (C) Steatosis was scored as percent of liver cells in 5, 10x fields per liver containing fat. (B) Representative photomicrographs of Oil Red O (neutral lipids, $\times 200$ ) stained frozen liver sections. (D) Image analysis of ORO-positive staining was performed using Image $\mathrm{J}$, and results are shown as percentage of microscope field. Results are reported as the mean \pm SD $(n=4-6) .{ }^{*}, p<0.05$ compared to group control; \#, p $<0.05$ compared to cadmium dose within diet group; @, p < 0.05 compared to corresponding normal diet. 
A
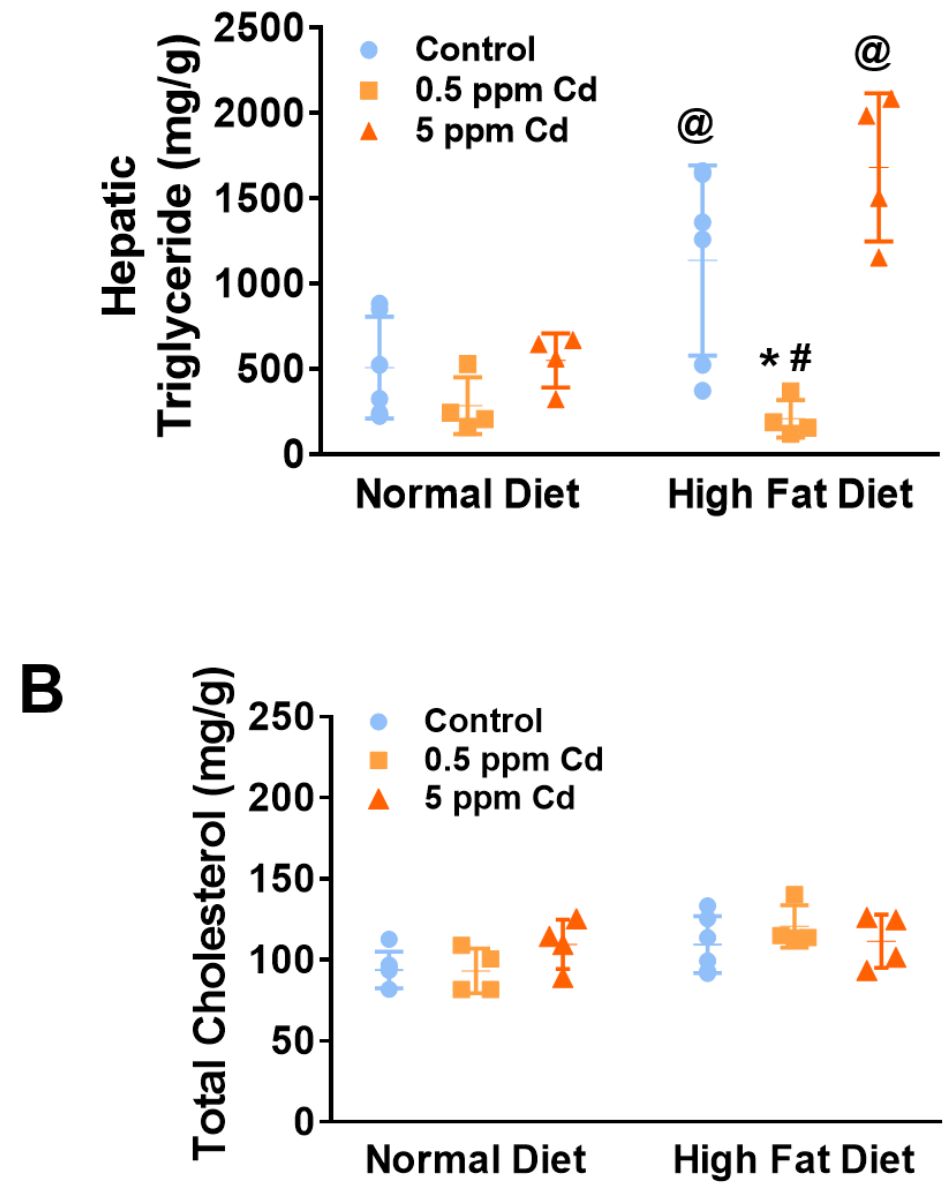

Figure 3.11. Effects of whole life cadmium exposure and high fat diet on biochemical measures of NAFLD in male mice. This figure shows cadmium exposure alters hepatic triglycerides in high fat diet fed mice, but not total cholesterol. (A) Hepatic triglyceride levels. (B) Total hepatic cholesterol levels. Results are reported as the mean $\pm S D(n=4-6) .{ }^{*}, p<0.05$ compared to group control; \#, $p<0.05$ compared to cadmium dose within diet group; @, $p<0.05$ compared to corresponding normal diet. 
regulatory element binding protein-1 (SREBP-1), which is responsible for the transcription of rate limiting lipogenic enzymes including fatty acid synthase (FASN) and stearoyl-CoA desaturase-1 (SCD-1) (Moslehi and Hamidi-Zad, 2018; Pei et al., 2020) contributes to the pathogenesis of NAFLD. Therefore, we assessed the levels of SREBP-1 in the liver.

SREBP-1 is first synthesized as inactive precursor tethered to the endoplasmic reticulum membrane and requires post-translational modification (i.e proteolytic cleavage) to produce its mature, transcriptionally active form (Ferre and Foufelle, 2007; Xiaoping and Fajun, 2012; Moslehi and Hamidi-Zad, 2018). Therefore we measured the cleaved, mature form of SREBP-1 (pro-SREBP-1) in addition to the full-length SREBP-1 (pro-SREBP-1). In control mice, consumption of high fat diet did not change mRNA or proteins levels of hepatic SREBP-1 (Figure 3.12A-E); however there was a decrease in the mRNA of two of SREBP-1's downstream targets, Fasn and Scd-1 (Figure 3.12F and G). In mice fed normal diet, cadmium exposure also did not significantly alter Srebf-1 mRNA or SREBP-1 protein levels in the liver (Figure 3.12A-E)

For high fat diet mice, although Srebf-1 mRNA levels were not significantly changed in mice exposed to $5 \mathrm{ppm}$ cadmium, SREBP-1 protein levels were significantly decreased compared to both high fat diet controls and $5 \mathrm{ppm}$ cadmium exposed mice fed normal diet (Figure 3.12A-E). This decrease in SREBP-1 protein levels was reflected in the mRNA levels of its downstream targets, Fasn and Scd1 (Figure 3.12F and G). 


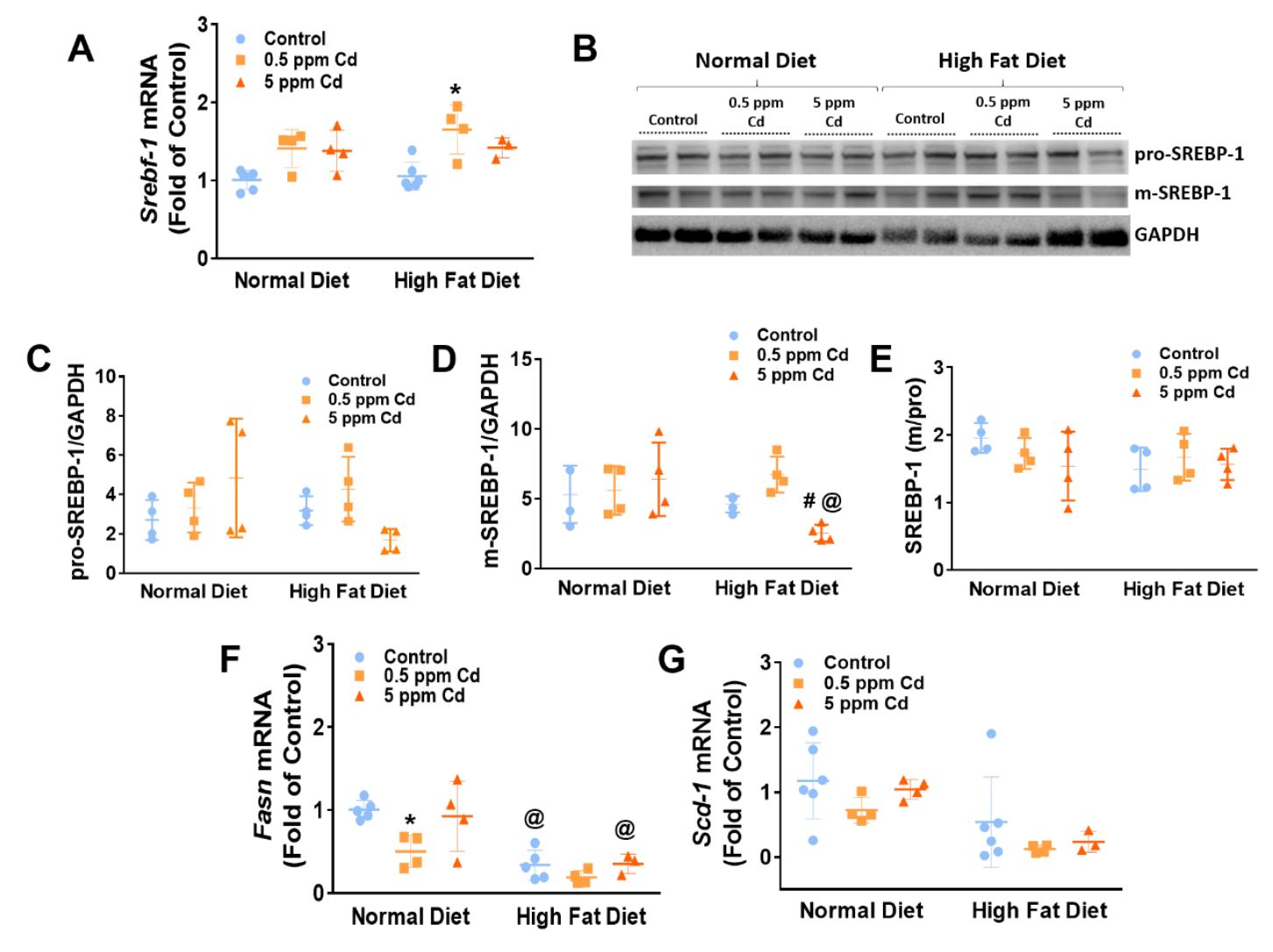

Figure 3.12. Effects of whole life cadmium exposure and high fat diet on SREBP-1 mediated lipogenesis. This figure shows cadmium-associated influences on high fat diet-induced NAFLD are not through SREBP-1 mediated lipogenesis. (A) Srebf-1 mRNA. (B) Representative western blot for hepatic SREBP-1 in all exposure groups. GAPDH was used as a loading control. Corresponding densitometric analyses shown as fold of control for (C) pro-SREBP1 (uncleaved; inactive), (D) mature (m)-SREBP-1 (cleaved; active) and (E) m/pro SREBP-1 ratio. mRNA levels for SREBP-1 downstream targets (F) Fasn and (G) Scd-1. Results are reported as the mean \pm SD $(n=4-6) .{ }^{*}, p<0.05$ compared to group control; \#, p < 0.05 compared to cadmium dose within diet group; @, p < 0.05 compared to corresponding normal diet. 
Interestingly, in mice fed high fat diet exposure to $0.5 \mathrm{ppm}$ cadmium significantly increased Srebf-1 mRNA levels compared to high fat diet fed controls, which was reflected in the protein levels of SREBP-1 (Figure 3.12A-E). However, the observed $0.5 \mathrm{ppm}$ cadmium-induced increases in SREBP-1 did not translate into increased Fasn or Scd-1 mRNA, both of which were expressed at levels similar to that of high fat diet fed controls (Figure 3.12F and G).

\section{Summary}

In this aim we first showed feeding male mice a high fat diet for 24 weeks post weaning caused weight gain over time, increased percent fat and lean tissue mass in the body and induced hyperglycemia while also increasing plasma insulin, indicative of insulin resistance. Furthermore, high fat diet caused liver injury, indicated by increased liver weight to tibia ratios and plasma ALT levels and significantly increased steatosis supported by oil red $\mathrm{O}$ and total hepatic triglyceride measurements. Taken together, we show that our model of high fat diet-induced NAFLD was successful.

We next showed our cadmium exposure model was successful. Cadmium accumulated in the liver in a concentration-dependent manner in mice exposed to cadmium. In mice exposed to the high concentration of cadmium (5 ppm), the cadmium accumulation was independent of diet. However, in mice exposed to the lower concentration of cadmium $(0.5 \mathrm{ppm})$, high fat diet feeding resulted in greater 
cadmium accumulation compared to those fed normal diet. Taken together, we show that our model of high fat diet-induced NAFLD was successful.

When we tested for cadmium effects on the hepatic expression of metallothionein, a key factor in cadmium detoxification, we found differing outcomes depending on the exposure. Hepatic metallothionein expression was assessed at both mRNA and protein levels. In high fat diet fed mice, $5 \mathrm{ppm}$ cadmium exposure dramatically lowered metallothionein response and exacerbated high fat diet-induced NAFLD. In contrast, in high fat diet fed mice, 0.5 ppm cadmium exposure increased the metallothionein response and rescued high fat diet-induced NAFLD.

\section{Aim 2. Show zinc supplementation protects against cadmium-enhanced,} high fat diet-induced NAFLD

\section{Background}

Zinc is an essential trace metal required for a variety of biological functions. More than 2,000 transcription factors and 300 enzymes require zinc for structural maintenance and appropriate functionality, indicating zinc is a key factor in numerous metabolic processes including glucose and lipid metabolism (Miao et al., 2013; Livingstone, 2015). Dysregulation of zinc homeostasis is associated with various metabolic disease pathologies (Seo et al., 2014). For example, zinc deficiency is common in obese, diabetic and NAFLD patients (Miao et al., 2013; Fukunaka and Fujitani, 2018; Shidfar et al., 2018) while zinc supplementation can reduce obesity, blood glucose levels and hepatic lipid deposition (Mahawar et al., 
2017; Thoen et al., 2019; Qi et al., 2020). Our laboratory showed zinc protects against diabetes-induced damage to multiple organs, including the liver and kidney, by increasing sensitivity to insulin and decreasing inflammation and oxidative stress (Li et al., 2014; Liang et al., 2015). In addition, we showed cardiac hypertrophy associated with high fat diet-induced obesity is exacerbated by zinc deficiency and rescued by zinc supplementation (Wang et al., 2016; Wang et al., 2017); however, the mechanism by which zinc offers protection against metabolic diseases is not completely understood.

The interactions between zinc and cadmium in the human body is considered to be one of the most well recognized and studied metal-metal interactions. Similarities between cadmium and zinc, such as they both tend to form tetrahedral complexes and are both group 2B transition metals that tend to form $2^{+}$charges, result in them having similar interactions with the human body (Peraza et al., 1998). Therefore, it is not surprising that one of the main mechanisms of cadmium toxicity involves cadmium-induced dysregulation of zinc metabolism and functionality; hence, cadmium is labeled a zinc antimetabolite (Cotzias and Papavasiliou, 1964; Peraza et al., 1998). Cadmium displaces zinc in numerous metallo-enzymes and zinc finger proteins, resulting in activity reduction of zinc containing enzymes and interference with essential metal homeostasis (Martelli et al., 2006; Namdarghanbari et al., 2014); thus, some symptoms of cadmium resemble those seen with zinc deficiency (Peraza et al., 1998).

Epidemiologically, increased intake of dietary zinc is associated with lower cadmium burden (Vance and Chun, 2015). Experimentally, zinc supplementation 
has been shown to alleviate symptoms of cadmium toxicity since the 1960's when Supplee (1961) showed cadmium toxicity in turkey poults (decrease growth rate and feather abnormalities) could be reversed with zinc supplements. Furthermore, zinc supplementation can counteract cadmium-induced inflammation, cell cycle dysregulation, and cadmium accumulation (Zhai et al., 2015; Zhang et al., 2014; Bonaventura et al., 2017). However, whether zinc supplementation can protect against cadmium-enhanced, high fat diet-induced liver disease is unknown, and its ability to rescue high fat diet-induced NAFLD, in general, is understudied.

In Aim 1, we showed our high fat diet model successfully induced NAFLD, our cadmium exposure model was effective, resulting in a concentration dependent increase in hepatic cadmium levels, and exposure to $5 \mathrm{ppm}$ cadmium in drinking water, starting in utero and continuing through adulthood, exacerbated high fat diet-induced liver disease. Therefore, following the same exposure paradigm used in Aim 1, we investigated the ability of zinc supplementation (90 mg zinc $/ 4057 \mathrm{kcal}$ ) to protect against cadmium-exacerbated, high fat diet-induced NAFLD (Figure 3.13)

\section{Findings}

\section{Impact of zinc supplementation on high fat diet-induced NAFLD}

Our first objective was to determine the impact of zinc supplementation on our high fat diet model using the same 9 measures used in Aim 1, first characterizing the model at the whole mouse level followed by investigation at the hepatic tissue level. Zinc supplementation did not influence weight gain over time 


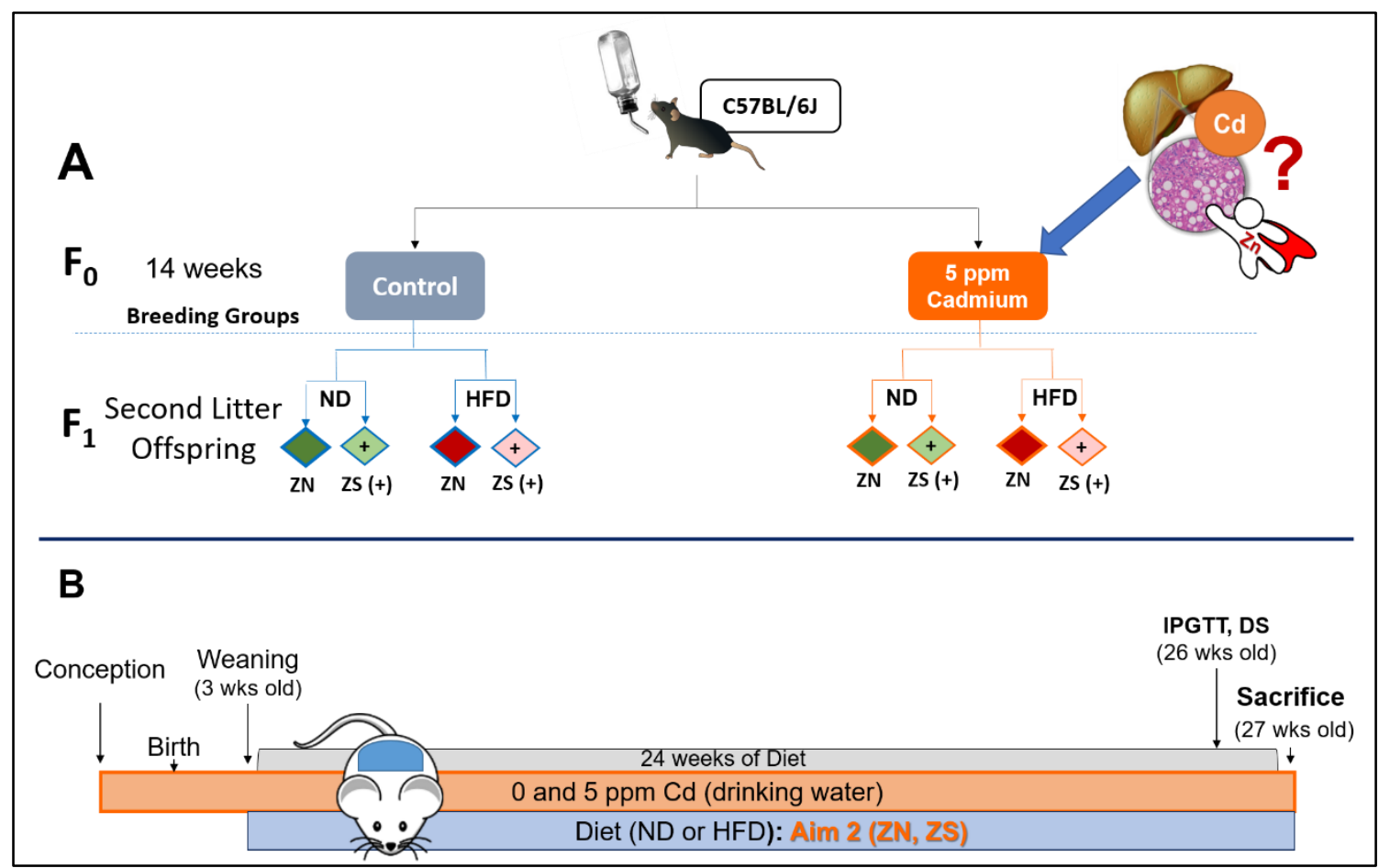

Figure 3.13. Model of whole life exposure of male mice to cadmium and high fat diet supplemented with zinc. Adult male and female C57BL/6J mice on defined diets were exposed to control drinking water, or water containing $5 \mathrm{ppm}$ cadmium for 14 weeks before being established into breeding pairs. Pregnant dams and offspring were continuously exposed and continued on the same drinking water regime as their parents after weaning. At weaning, male offspring were also fed either a normal or high-fat diet (ND or HFD, respectively) containing 30 or $90 \mathrm{mg}$ zinc/4057 kcal, representing normal zinc (ZN) and zinc supplemented (ZS) diet, respectively, for 24 weeks. The same paradigm will be used in Aim 3 with the modulation of zinc in the diet. One week prior to sacrifice, IPGTT tests were performed and body composition determine by DEXAscan (DS). IPGTT = intra-peritoneal glucose tolerance test, wks $=$ weeks . 
A

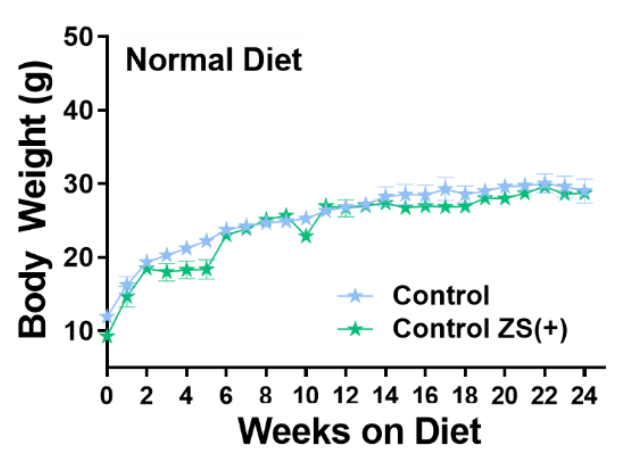

C

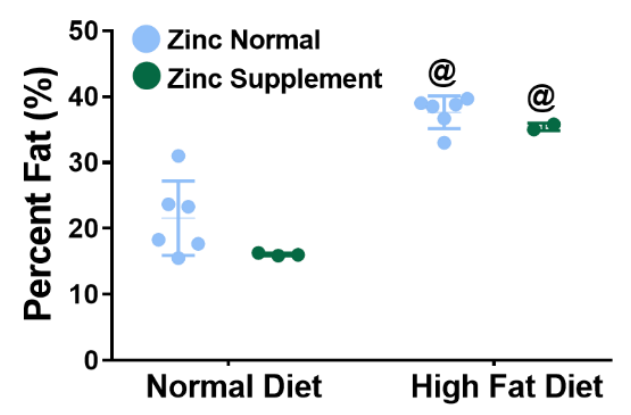

$\mathbf{E}$

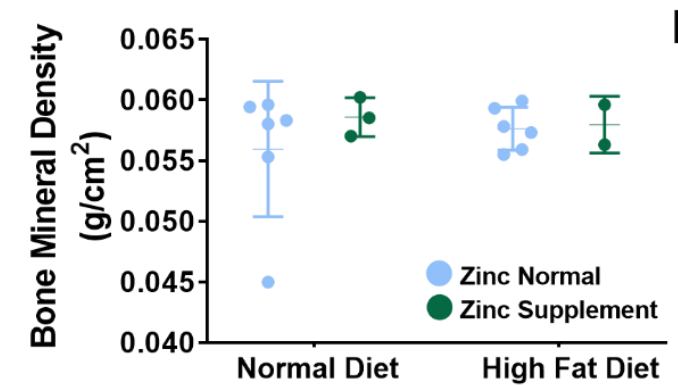

B
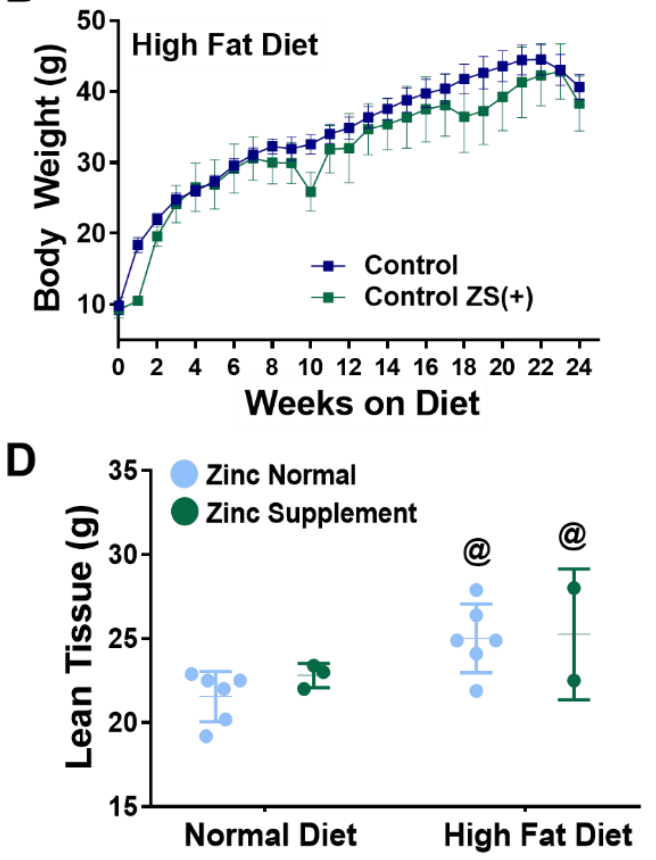

F

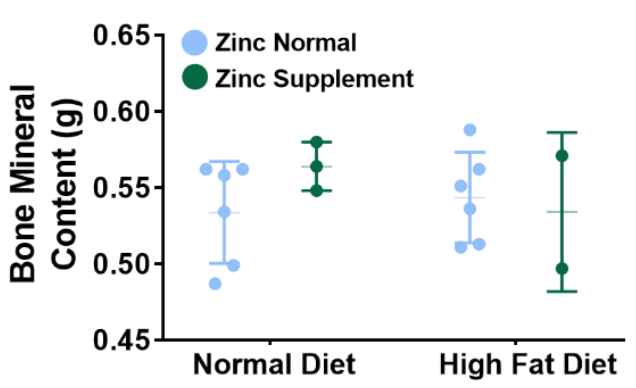

Figure 3.14. Effects of zinc supplementation and high fat diet on body weight and composition in male mice. This figure shows zinc supplementation did not alter body weight or body composition in male mice after 24 weeks of diet, post weaning. Weekly body weight in (A) normal diet fed mice and (B) high fat diet fed mice. DEXAscan was performed one week prior to sacrifice for control and $5 \mathrm{ppm}$ cadmium exposed mice to assess body composition changes. (C) Percent fat, (D) lean tissue mass, $(E)$ bone mineral density and $(F)$ bone mineral content. Results are reported as the mean \pm SD $(n=2-6)$ for DEXAscan and mean \pm SEM $(n=2-6)$ for body weights. @, p $<0.05$ compared to normal diet. 
in normal or high fat diet-fed mice (Figure 3.14A and B) nor did it alter DEXAscan measured parameters of body composition: percent fat, lean tissue mass, bone mineral density or bone mineral content (Figure 3.14C-F). Therefore this finding indicates that in our model, zinc supplementation did not attenuate high fat dietinduced weight gain or fat mass. Following a glucose challenge, zinc supplementation did not alter glucose uptake in normal diet-fed mice (Figure 3.15A and C); however, in mice consuming high fat diet, zinc supplementation tended to decrease blood sugar levels (Figure 3.15B and C). These results were also reflected in the plasma insulin levels (Figure 3.15D). Zinc supplementation tended to reduce hepatic injury resulting from high fat diet as measured by liver weight to tibia length ratios (Figure 3.16A) and plasma ALT (Figure 3.16B), but not AST (Figure 3.16C). Histological analysis of the liver by H\&E (Figure 3.17A and B) and oil red $\mathrm{O}$ staining (Figure 3.17C and D) showed zinc supplementation decreased steatosis and reduced staining of neutral lipids. In addition, hepatic triglycerides were reduced as a result of zinc supplementation (Figure 3.17E). These results indicate zinc supplementation tended to rescue high fat diet-induced liver damage without influencing weight gain or body composition.

\section{Impact of zinc supplementation on general cadmium measures}

Next, we investigated the impact of zinc supplementation on our cadmium exposure model. We first measured hepatic zinc levels in order to determine if the addition of zinc to the diet translated to increased zinc in the liver. In mice fed normal diet, zinc supplementation did not change hepatic zinc levels in control or 
A

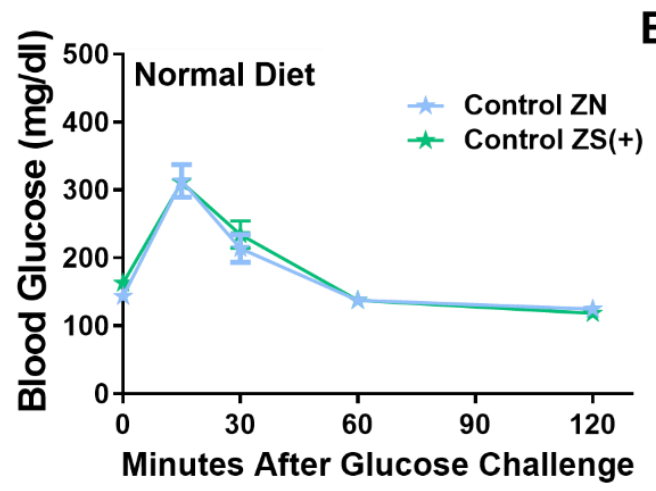

C

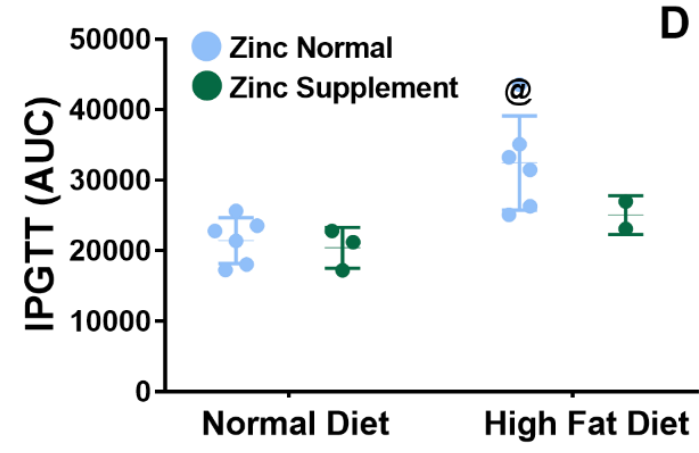

B
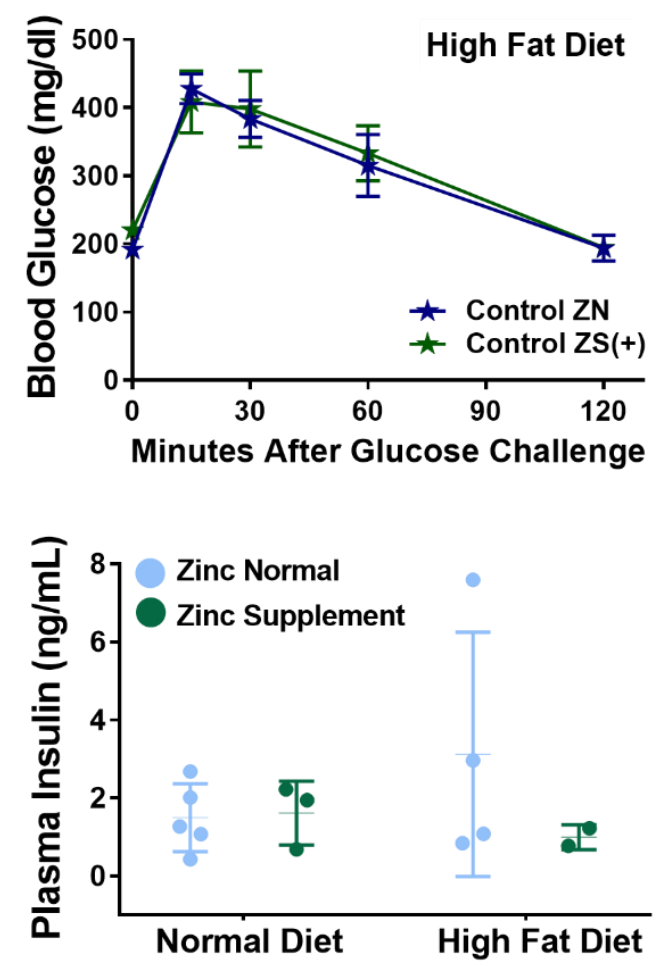

Figure 3.15. Effects of zinc supplementation and high fat diet on glucose handling and plasma insulin in male mice. This figure shows zinc supplementation tended to decrease high fat diet-associated glucose intolerance and plasma insulin levels, although statistical significance was observed. A) Blood glucose levels in normal diet-fed mice and (B) high fat diet-fed mice after IPGTT, performed one week prior to sacrifice. (C) Integrated area under the curve (AUC) showing quantitative changes in blood glucose levels after glucose challenge. (D) Insulin levels in plasma at time of sacrifice. Results are reported as the mean \pm SD (n=2-6). @, $\mathrm{p}<0.05$ compared to normal diet. 


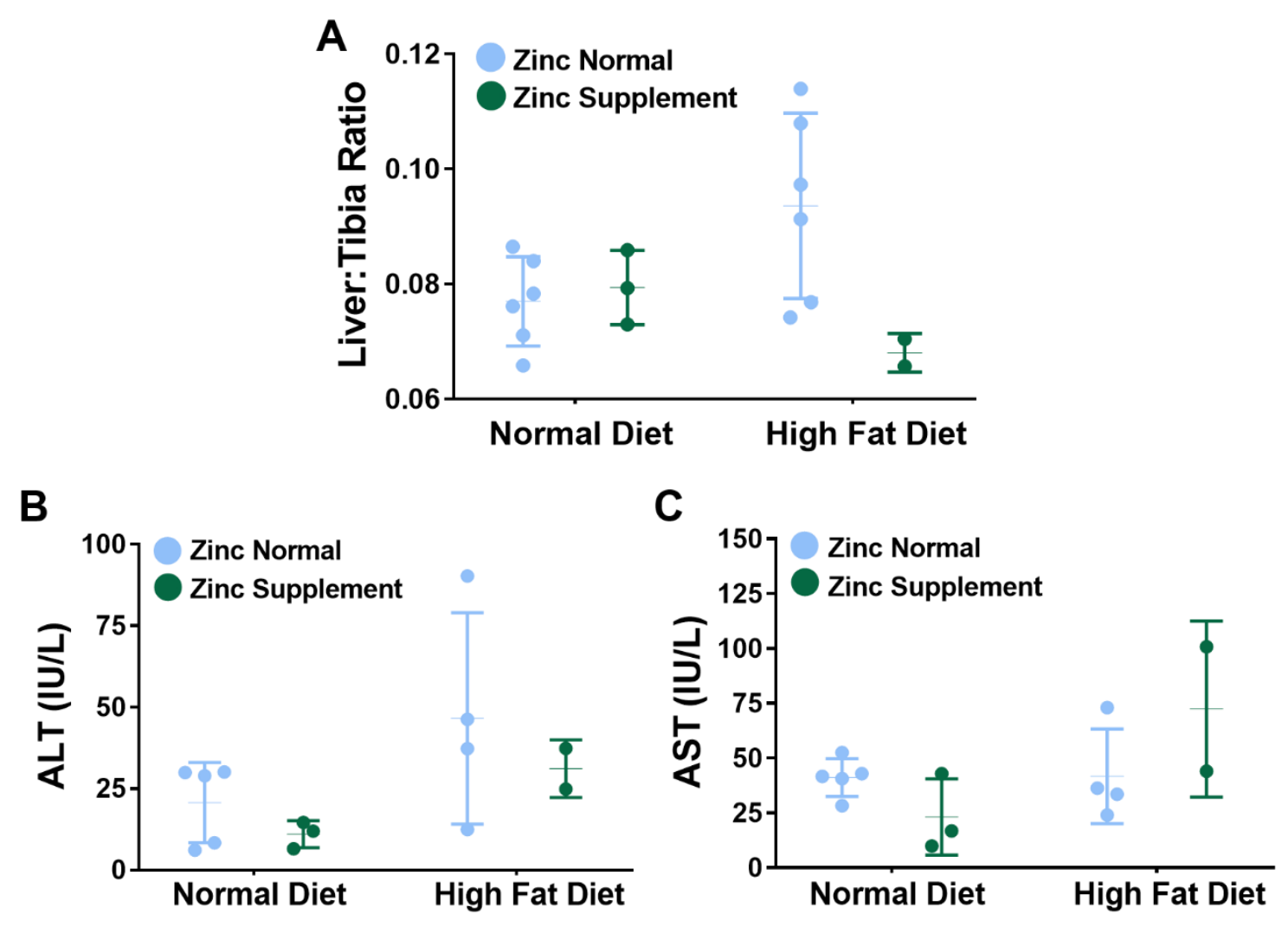

Figure 3.16. Effects of zinc supplementation and high fat diet on liver injury in male mice. This figure shows zinc supplementation tended to decrease liver injury although no statistical significance was observed. (A) Ratio of liver weight in grams to tibia length in millimeters, a measure of hepatomegaly. (B) Plasma aspartate aminotransferase (AST) and (C) plasma alanine aminotransferase (ALT) activity. Results are reported as the mean $\pm S D(n=2-6)$. 
A
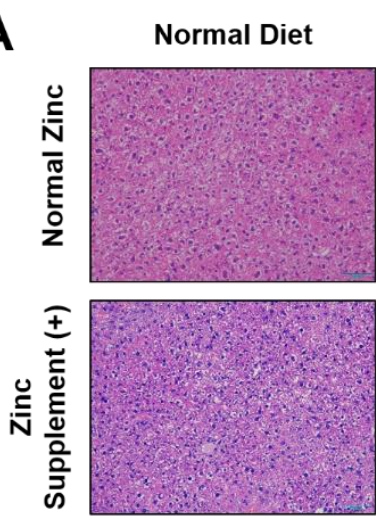

C

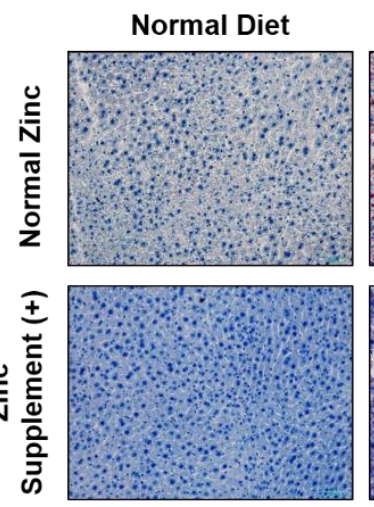

High Fat Diet
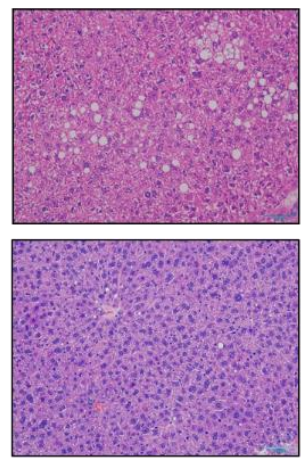

High Fat Diet
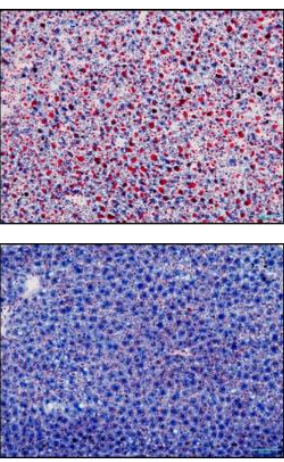

B

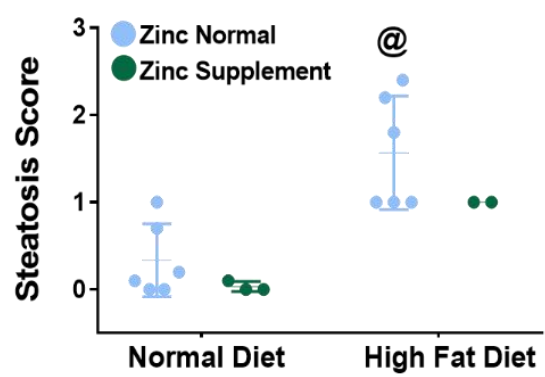

D

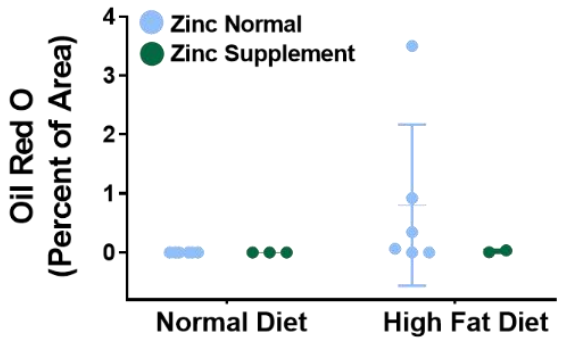

$\mathbf{E}$

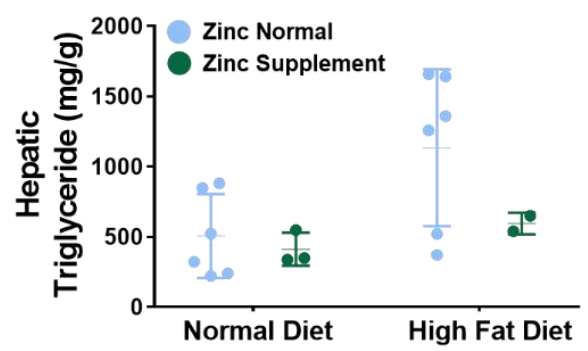

Figure 3.17. Effects of zinc supplementation and high fat diet on NAFLD in male mice. This figure shows zinc supplementation tended to decrease high fat diet-induced NAFLD, although no statistical significance was observed. (A) Representative photomicrographs of paraffin embedded liver tissue stained with hematoxylin \& eosin staining (H\&E, 200x). (C) Steatosis was scored as percent of liver cells in 5, 10x fields per liver containing fat. (B) Representative photomicrographs of Oil Red O (neutral lipids, $\times 200$ ) stained frozen liver sections. (D) Image analysis of ORO-positive staining was performed using Image $\mathrm{J}$, and results are shown as percentage of microscope field. (E) Biochemical quantification of hepatic triglycerides. Results are reported as the mean $\pm S D(n=2$ 6). @, p $<0.05$ compared to corresponding normal diet. 
A

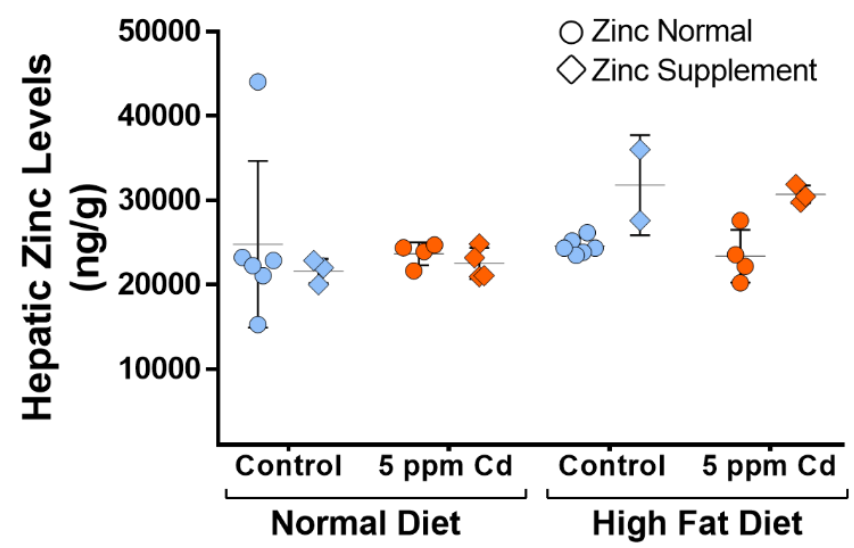

B

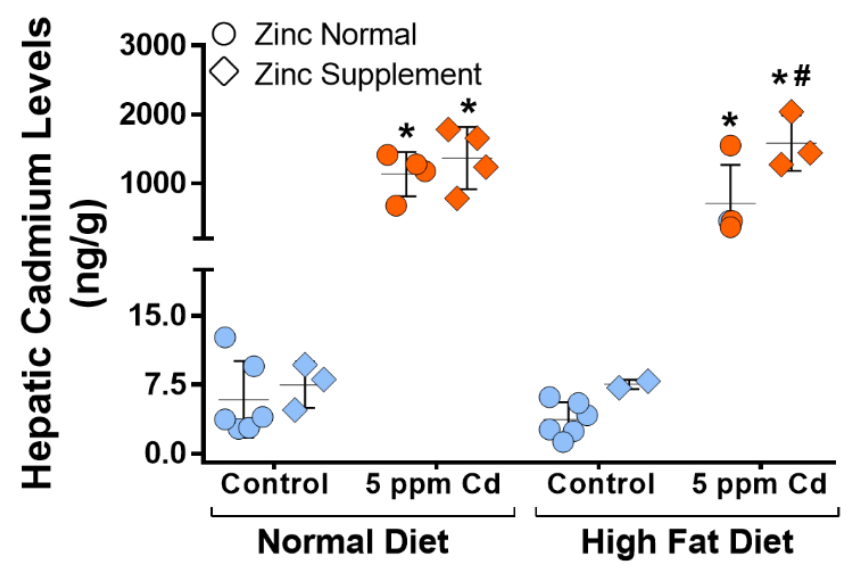

C

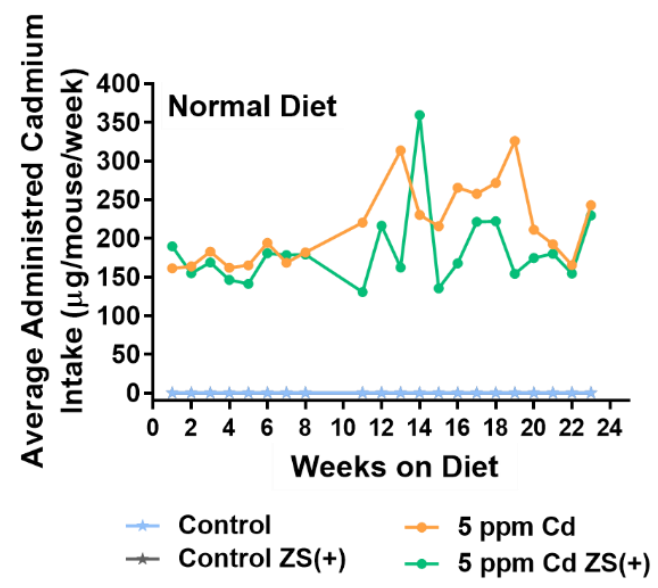

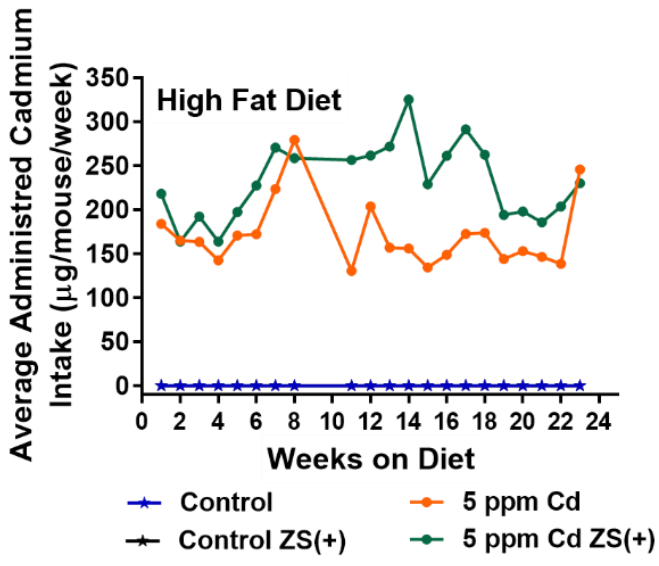

Figure 3.18. Hepatic cadmium and zinc levels and weekly administered cadmium intake in zinc supplemented male mice. This figure shows zinc supplementation increased zinc and cadmium accumulation in high fat diet fed 
male mice, reflecting the average administered cadmium intake delivered by drinking water. (A) Hepatic zinc levels measured by ICP-MS. (B) Hepatic cadmium levels measured by ICP-MS. (C) Average weekly administered cadmium intake delivered by drinking water in zinc supplemented, normal diet fed mice. (D) Average weekly administered cadmium intake delivered by drinking water in in zinc supplemented, high fat diet fed mice. ${ }^{*}, p<0.05$ compared to group control; \#, $p<$ 0.05 compared to cadmium dose within diet group. 
cadmium exposed mice (Figure 3.18A). In contrast, although not statistically significant, in high fat diet-fed mice, mean hepatic zinc levels increased approximately $30 \%$ in both control and cadmium exposed animals, respectively, as a result of dietary zinc supplementation. Dietary zinc supplementation did not change cadmium levels in the liver of neither control nor 5 ppm cadmium exposed mice fed normal diet (Figure 3.18B). In mice fed high fat diet, zinc supplementation slightly increased cadmium levels in control mice whereas in mice exposed to 5 ppm cadmium, mean hepatic cadmium levels $($ cadmium $=1584 \mathrm{ng} / \mathrm{g}$ ) were more than double those of mice with a normal amount of zinc in their diet (cadmium = $705 \mathrm{ng} / \mathrm{g}$ ), suggesting increased intake of dietary zinc provides a mechanism for greater cadmium accumulation in the liver in addition to greater hepatic zinc levels.

Mice were exposed to cadmium in their drinking water, thus we measured water consumption and calculated the average administered cadmium intake to determine zinc supplementation altered cadmium intake (Figure 3.18B and C). Over the course of the study, 5 ppm cadmium exposed mice fed zinc supplemented normal or high fat diet drank an average of $5.24 \pm 1.41$ and $6.62 \pm$ $1.23 \mathrm{~mL}$ of water per a day in normal and high, respectively and these results were not significantly different from the corresponding mice fed diets with normal zinc. Therefore, cadmium exposed mice drank similar amounts of water independent of dietary zinc or fat levels. The average daily dose of cadmium in zinc supplemented mice that drank water with $5 \mathrm{ppm}$ cadmium was $26.18 \pm 7.07$ and $33.08 \pm 6.15 \mu \mathrm{g}$ in normal and high fat diet fed mice, respectively. Interestingly, in high fat diet fed mice the average daily dose of cadmium in $5 \mathrm{ppm}$ cadmium exposed mice 
supplemented with zinc was significant greater than in mice with normal zinc $(p<0.0001)$. Overall, zinc supplementation did not change water consumption, but did increase cadmium uptake in high fat diet fed animals.

In addition to being one of the principal detoxification mechanisms against cadmium toxicity, metallothionein is crucial in the physiological maintenance of zinc homeostasis (Funk et al., 1987). Synthesis of metallothionein is induced by the presence of both zinc and cadmium. Therefore, one of the reasons for the protective effects of zinc supplementation against cadmium toxicity is through the production of metallothionein (Stonard and Webb, 1976; Peraza et al., 1998). We investigated the impact of zinc supplementation on hepatic metallothionein levels in our model. Zinc supplementation significantly reduced the cadmium-induced increases in mRNA levels of metallothionein in normal diet fed mice found in Aim 1 (Figure 3.19A and B). In high fat diet fed mice, zinc supplementation significantly increased metallothionein mRNA in controls, but not in cadmium exposed mice (Figure 3.19A and B). Overall, the mRNA levels of the two isoforms of metallothionein (Mt-1 and Mt-2) correlated at both the exposure group and individual mouse level. However, the mRNA levels of Mt-2 were approximately 2 times greater than $M t-1$ levels in both high fat diet fed control animals supplemented with zinc and in normal diet fed animals exposed to $5 \mathrm{ppm}$ cadmium.

We also assessed hepatic metallothionein protein levels. We found in mice supplemented with zinc, high fat diet changed metallothionein protein levels in control animals, but not in cadmium exposed animals compared to mice consuming a normal diet (Figure 3.19C and D). We also found in high fat diet fed 

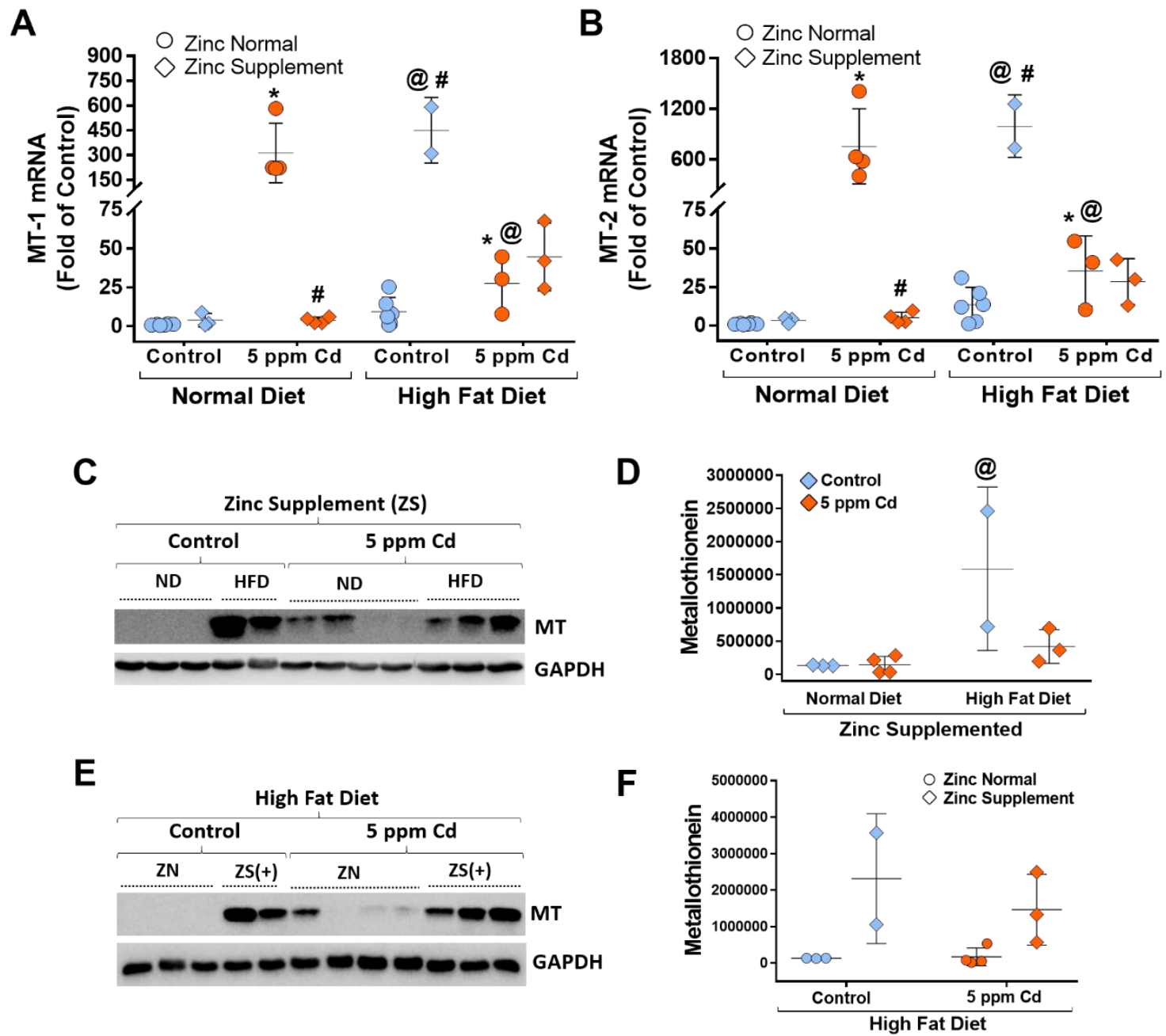

Figure 3.19. Effects of zinc supplementation, cadmium exposure and high fat diet on metallothionein (MT) in male mice. (A) Hepatic mRNA expression of MT1 and (B) MT-2. (C) Representative western blot for hepatic MT in zinc supplemented mice and (D) corresponding densitometric analyses shown as fold of control. (E) Representative western blot for hepatic MT in high fat diet fed mice and $(F)$ corresponding densitometric analyses shown as fold of control. GAPDH was used as a loading control. Results are reported as the mean $\pm S D(n=2-6)$. *, $\mathrm{p}<0.05$ compared to group control; \#, $\mathrm{p}<0.05$ compared to cadmium dose within diet group; @, p < 0.05 compared to corresponding normal diet. 
mice zinc supplementation increased metallothionein protein levels in both control and cadmium exposed mice compared to mice consuming a high fat diet with normal zinc (Figure 3.19E and F). At both an individual mouse and exposure group level metallothionein mRNA levels correlate with protein levels. Thus, overall, as evidenced by both mRNA and protein levels, zinc supplementation in high fat diet fed mice increased metallothionein levels in both control and cadmium exposed mice; therefore indicating a possible mechanism by which zinc supplementation may attenuate cadmium-exacerbated, high fat diet-induced NAFLD.

\section{Impact of zinc supplementation on cadmium-exacerbated, high fat diet- induced NAFLD}

In Aim 1, we found exposure to 5 ppm cadmium did not influence body weight gain in normal diet-fed mice and we found that zinc supplementation did not change this result (Figure 3.20A). In contrast, we reported exposure to $5 \mathrm{ppm}$ cadmium enhanced high fat diet-induced weight gain and found zinc supplementation significantly attenuated this weight gain to levels below those found in high fat diet control mice $(p=0.001$, Figure 3.20B). Due to budgetary restraints, DEXAscan was not performed on normal diet fed, zinc supplemented mice exposed to $5 \mathrm{ppm}$ cadmium. However, our hypothesis is centered on zinc supplementation rescuing high fat diet-induced effects; thus these data are useful, showing zinc supplementation significantly reduced the percentage of fat in $5 \mathrm{ppm}$ cadmium exposed mice fed high fat diet (Figure 3.20C) although lean tissue, bone mineral density and bone mineral content were unchanged (Figure 3.20D-F). 

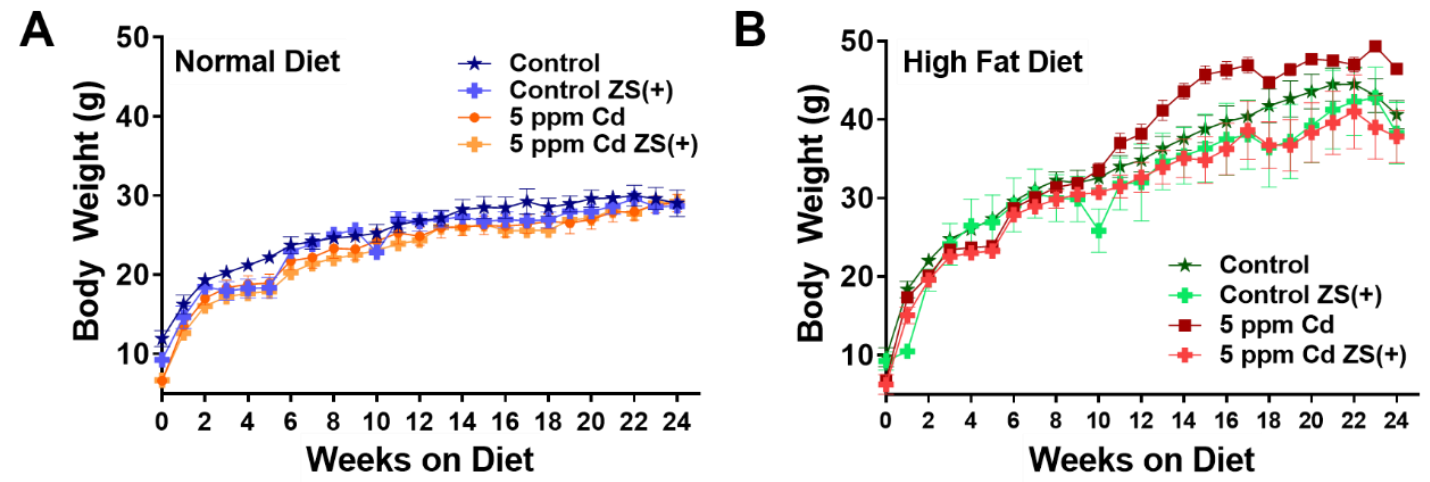

C

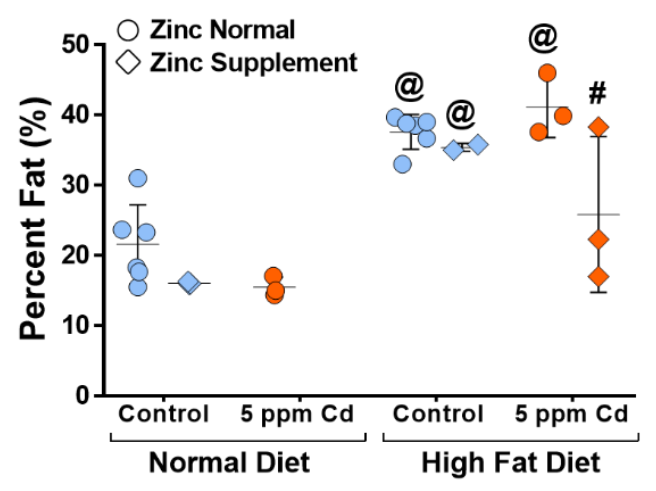

E

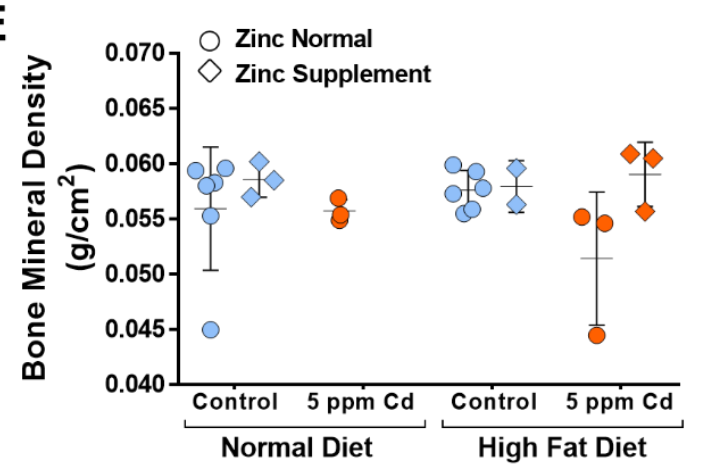

D

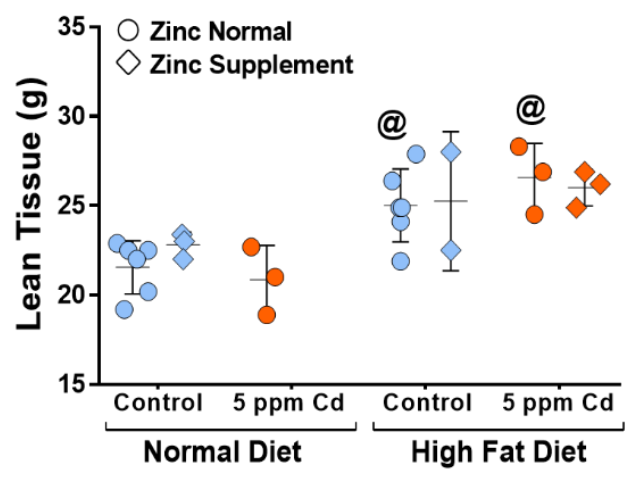

F

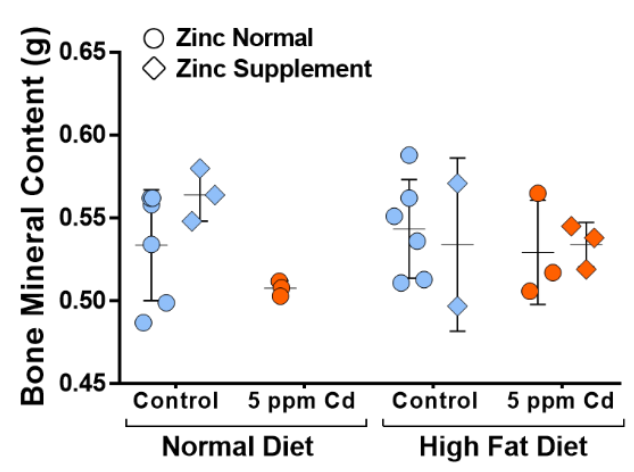

Figure 3.20. Effects of zinc supplementation on cadmium-exacerbated, high fat diet-induced weight gain and body composition in male mice. Zinc supplementation reduced body weight gain in high fat diet fed mice exposed to 5 ppm cadmium as well as percent fat. Body weight was measured once a week for 24 weeks starting at weaning, through until sacrifice. Weekly body weight in (A) normal diet fed male mice and (B) high fat diet fed mice with or without zinc supplementation. DEXAscan was performed one week prior to sacrifice for control 
and 5 ppm cadmium exposed mice to assess body composition changes. (C) Percent fat, $(D)$ lean tissue mass, $(E)$ bone mineral density and $(F)$ bone mineral content. Results are reported as the mean \pm SD for DEXAscan $(n=2-6)$ and mean \pm SEM $(n=2-6)$ for body weights. \#, $p<0.05$ compared to corresponding normal zinc diet; @, $p<0.05$ compared to corresponding normal diet. 
A
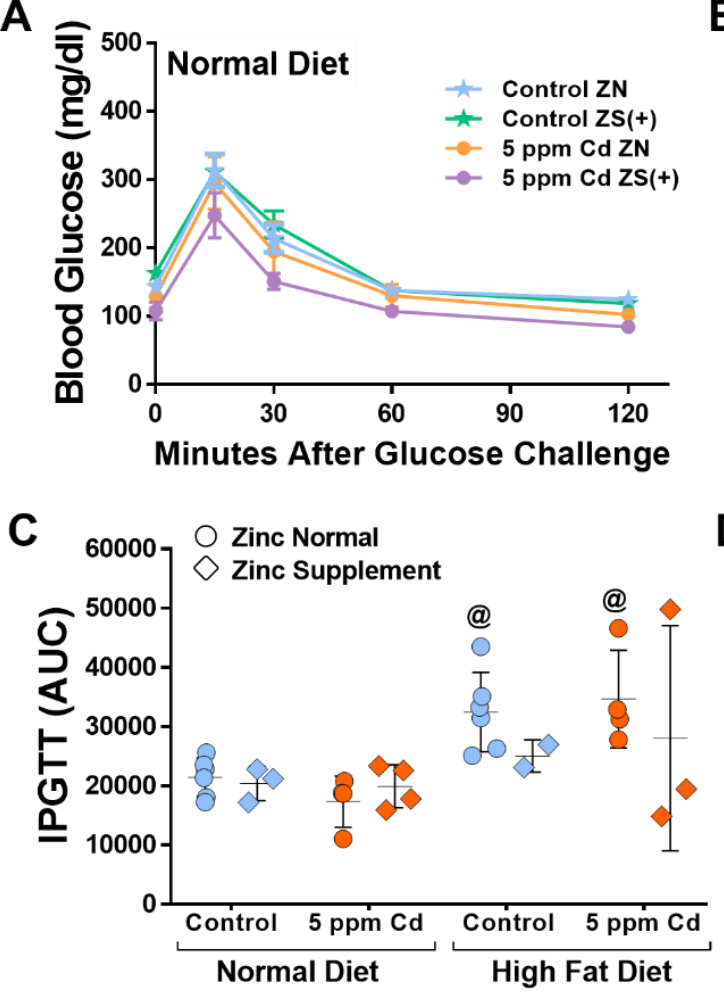

B

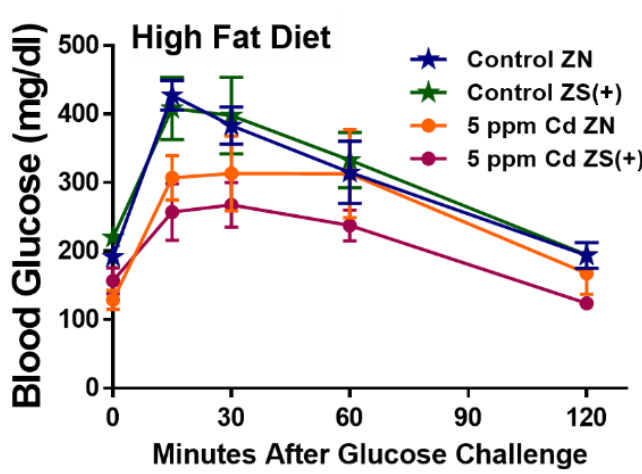

D I ${ }_{8} \circ$ Zinc Normal

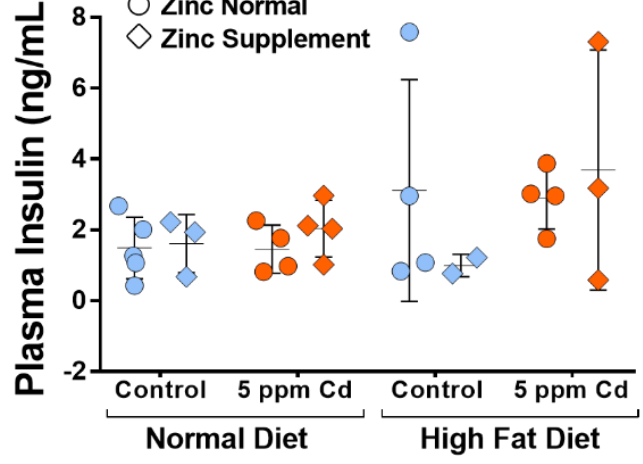

Figure 3.21. Effects of zinc supplementation on cadmium and high fat dietaltered glucose handling and plasma insulin in male mice. Zinc supplementation tended to reduce glucose intolerance in high fat diet fed mice, independent of cadmium exposure. Blood glucose levels in (A) normal diet fed and (B) high fat diet fed mice after IPGTT, performed one week prior to sacrifice. (C) Integrated area under the curve (AUC) showing quantitative changes in blood glucose levels after glucose challenge. (D) Insulin levels in plasma at time of sacrifice. Results are reported as the mean \pm SD $(n=2-6)$. @, $p<0.05$ compared to corresponding normal diet. 
In addition, similar to what was observed in high fat diet-fed control animals, zinc supplementation tended to decrease blood sugar levels in the $5 \mathrm{ppm}$ cadmium exposed animals fed high fat diet (Figure 3.21A-C). However, the ability of additional zinc to attenuate these cadmium-exacerbated, high-fat-diet-induced outcomes was not reflected in the plasma insulin levels (Figure 3.21D). Taken together, these data suggest zinc supplementation can attenuate the enhanced high fat diet-induced outcomes, at least at the whole animal level, resulting from cadmium exposure.

Therefore, the next step was to determine if zinc supplementation could rescue the liver injury and worsened steatosis observed in our model as a result of cadmium exposure in combination with high fat diet. Indeed, liver injury was significantly reduced in high-fat-diet-fed, cadmium-exposed mice when given additional zinc in their diet (Figure 3.22A-C). Furthermore, zinc supplementation significantly reduced both steatosis (Figure $3.23 \mathrm{~A}$ and $\mathrm{C}$ ) and neutral lipids (Figure 3.23B and D) in cadmium exposed animals fed high fat diet as well as significantly decreased hepatic triglycerides (Figure 3.24) compared to the corresponding mice with normal zinc levels in their diet. 
A

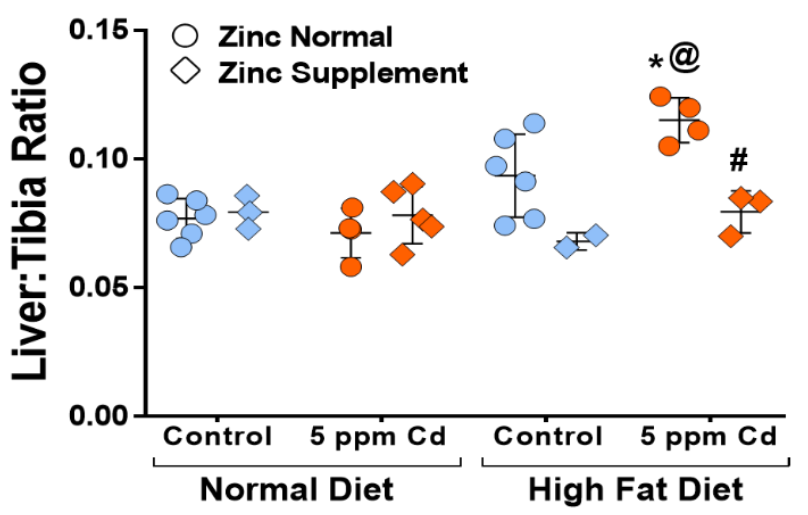

B

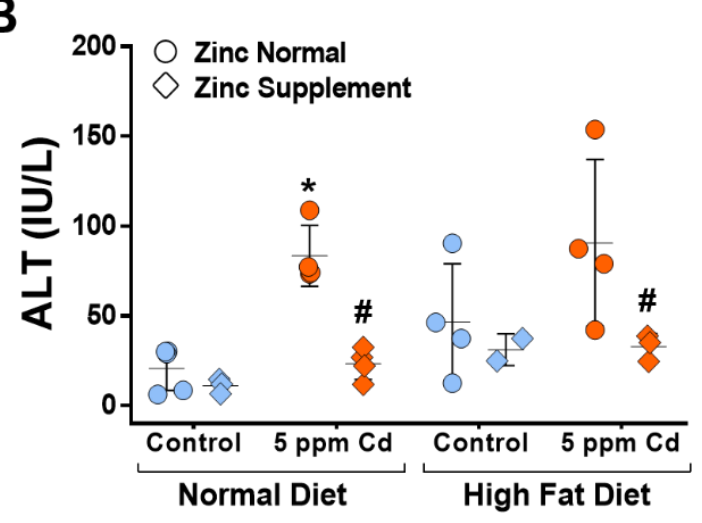

C

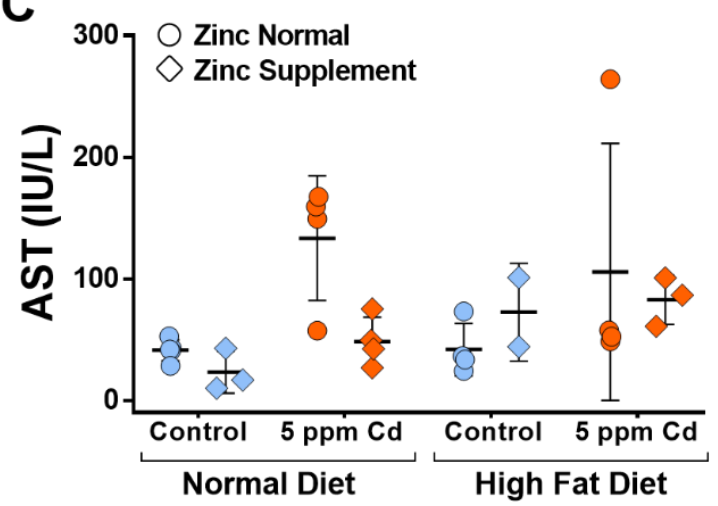

Figure 3.22. Zinc supplementation recues cadmium-exacerbated, high fat diet-induced liver injury in male mice. (A) Ratio of liver weight in grams to tibia length in millimeters, a measure of hepatomegaly. (B) Plasma aspartate aminotransferase (AST) and (C) plasma alanine aminotransferase (ALT) activity. Results are reported as the mean $\pm S D(n=2-6) .{ }^{*}, p<0.05$ compared to group control; \#, p $<0.05$ compared to corresponding normal zinc diet; @, $p<0.05$ compared to corresponding normal diet. 
A
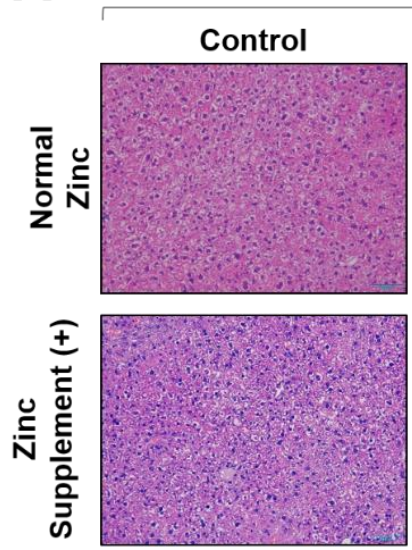

B
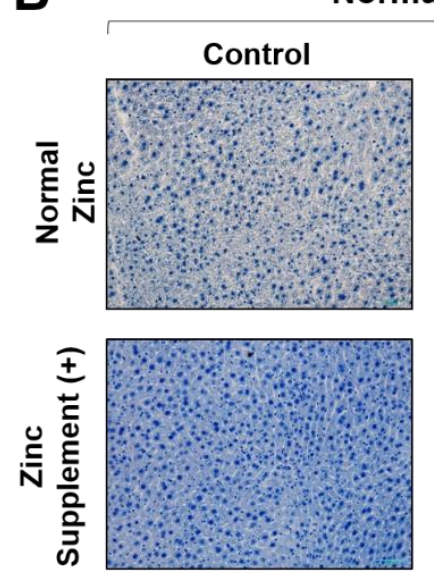

C

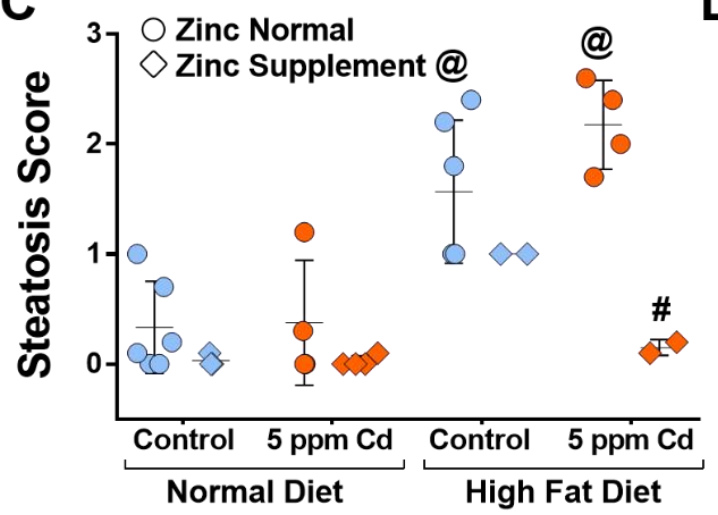

High Fat Diet
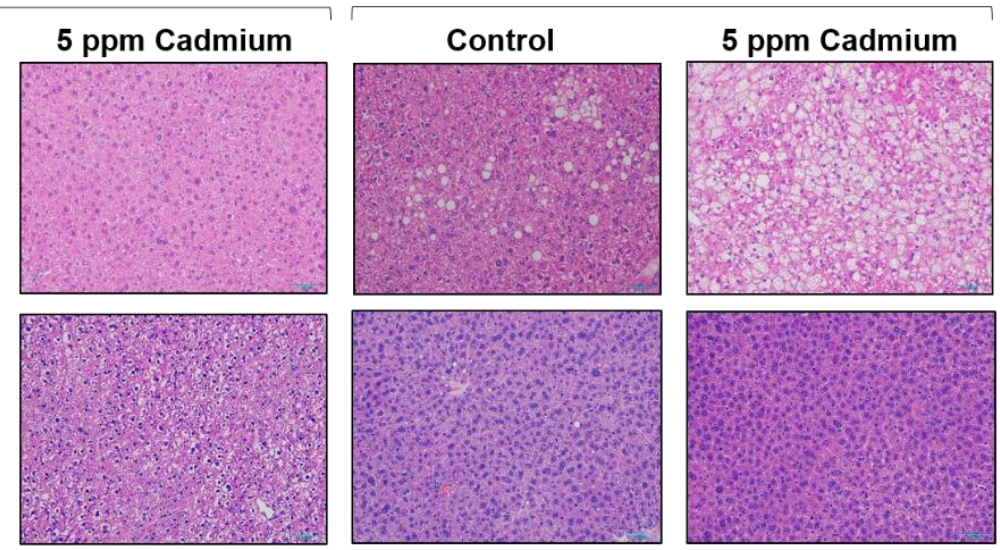

High Fat Diet
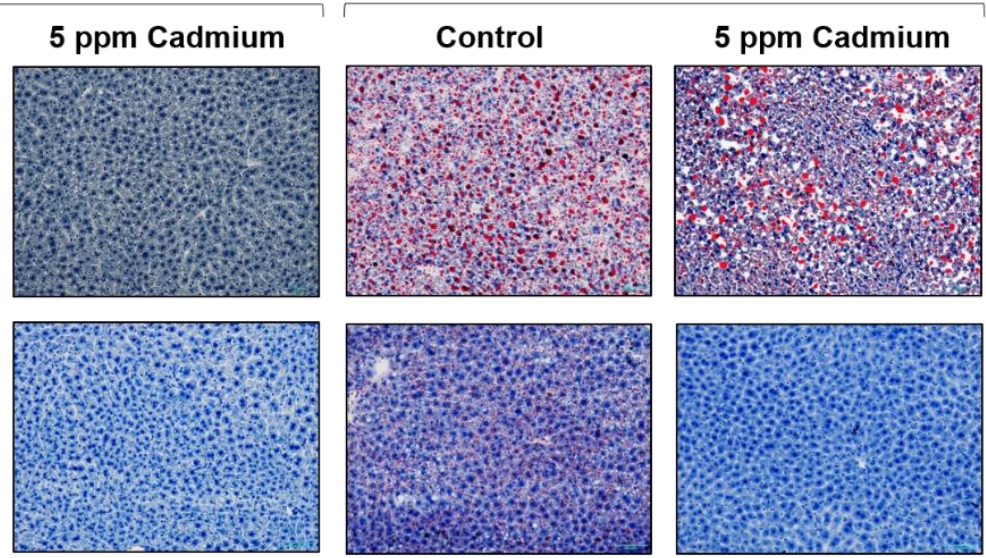

D

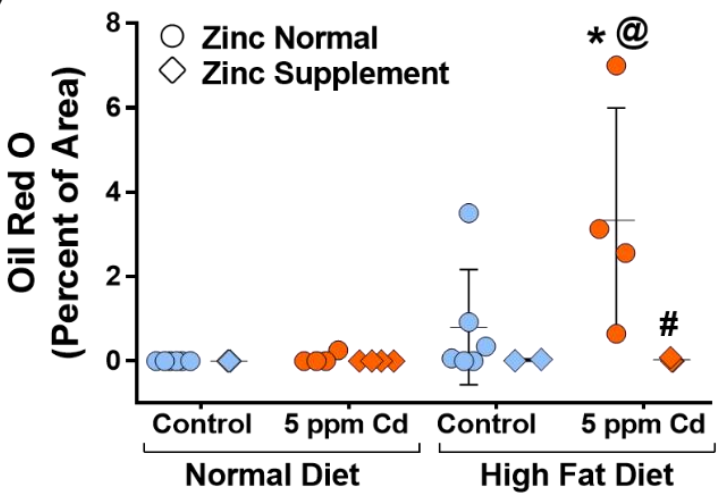

Figure 3.23. Zinc Supplementation recues cadmium-exacerbated, high fat diet-induced NAFLD in male mice. (A) Representative photomicrographs of paraffin embedded liver tissue stained with hematoxylin \& eosin staining $(\mathrm{H} \& \mathrm{E}$, 
200x). (C) Steatosis was scored as percent of liver cells in 5, 10x fields per liver containing fat. (B) Representative photomicrographs of Oil Red O (neutral lipids, $\times 200)$ stained frozen liver sections. (D) Image analysis of ORO-positive staining was performed using Image $\mathrm{J}$, and results are shown as percentage of microscope field. Results are reported as the mean $\pm S D(n=2-6) .{ }^{*}, p<0.05$ compared to group control; \#, $p<0.05$ compared to corresponding normal zinc diet; @, $p<0.05$ compared to corresponding normal diet. 


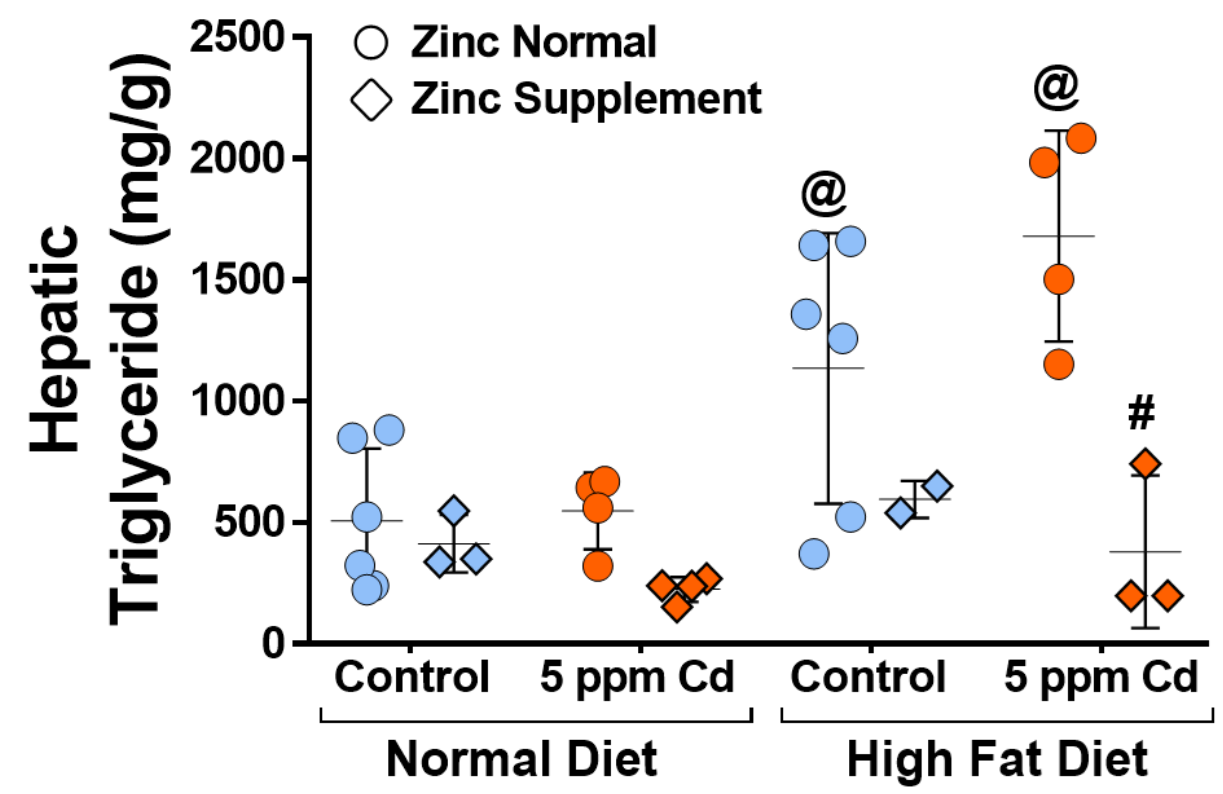

Figure 3.24. Zinc supplementation recues cadmium-exacerbated, high fat diet-induce hepatic triglycerides in male mice. Results are reported as the mean $\pm S D(n=2-6)$. \#, $p<0.05$ compared to corresponding normal zinc diet; $@, p$ $<0.05$ compared to corresponding normal diet. 


\section{Summary}

The results of this Aim show zinc supplementation did impact normal dietfed control mice. However, in high fat diet-fed mice zinc supplementation could marginally attenuate NAFLD in control animals as indicated by trends of decreasing hyperglycemia, hepatomegaly, ALT, steatosis, lipid deposition and hepatic triglycerides (Table 3.1).

Table 3.1. Summary of the effects of zinc supplementation in control mice.

\begin{tabular}{|c|c|c|c|c|}
\hline & \multicolumn{4}{|c|}{ Control Males } \\
\hline & \multicolumn{2}{|c|}{ Normal Diet } & \multicolumn{2}{|c|}{ High Fat Diet } \\
\hline & Normal Zinc & $\begin{array}{c}\text { Zinc } \\
\text { Supplementation }\end{array}$ & Normal Zinc & $\begin{array}{c}\text { Zinc } 5 \\
\text { Supplementation }\end{array}$ \\
\hline \multicolumn{5}{|c|}{ Whole Animal Characterization } \\
\hline Body Weight & - & - & 个个 & 个个 \\
\hline$\%$ Fat & - & $\downarrow$ & 个个 & $\downarrow$ \\
\hline IPGTT AUC & - & - & 个个 & $\downarrow$ \\
\hline Plasma Insulin & - & - & $\uparrow$ & $\downarrow$ \\
\hline \multicolumn{5}{|c|}{ NAFLD Characterization } \\
\hline Liver:Tibia Ratio & - & - & 个 & $\downarrow$ \\
\hline ALT & - & - & $\uparrow$ & $\uparrow$ \\
\hline AST & - & - & - & - \\
\hline Steatosis & - & - & 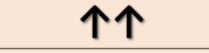 & $\downarrow$ \\
\hline Oil Red O & - & - & $\uparrow$ & $\downarrow$ \\
\hline Hepatic Triglycerides & - & - & 个个 & $\downarrow$ \\
\hline
\end{tabular}

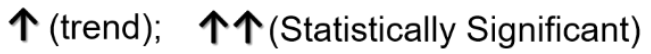

By contrast, zinc supplementation had a much stronger effect on cadmium exposed animals. Zinc supplementation increased hepatic zinc, cadmium and metallothionein levels in high fat diet-fed mice, but not normal diet fed mice (Figures 3.18 and 3.19). Supplementation also attenuated cadmium exacerbated, high fat diet-induced NAFLD by significantly decreasing hepatomegaly, plasma 
ALT levels, steatosis, lipid deposition and hepatic triglycerides (Table 3.2). Overall, these data implicate the use of zinc supplementation as a possible therapeutic for NAFLD, which may in part act by targeting metallothionein.

Table 3.2. Summary of zinc supplementation effects in cadmium exposed, high fat diet fed mice.

\begin{tabular}{|c|c|c|c|c|}
\hline & \multicolumn{4}{|c|}{ High Fat Diet Males } \\
\hline & \multicolumn{2}{|c|}{ Control } & \multicolumn{2}{|c|}{5 ppm Cd } \\
\hline & Normal Zinc & $\begin{array}{c}\text { Zinc } \\
\text { Supplementation }\end{array}$ & Normal Zinc & $\begin{array}{c}\text { Zinc } \sqrt{4} \\
\text { Supplementation }\end{array}$ \\
\hline \multicolumn{5}{|c|}{ Whole Animal Characterization } \\
\hline Body Weight & 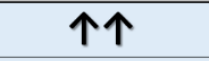 & 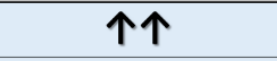 & 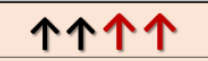 & $\downarrow \downarrow$ \\
\hline$\%$ Fat & 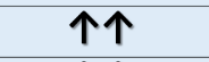 & $\downarrow$ & 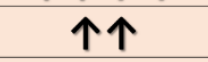 & $\downarrow$ \\
\hline IPGTT AUC & 个个 & $\downarrow$ & 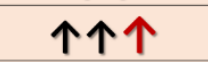 & $\downarrow$ \\
\hline Plasma Insulin & $\uparrow$ & $\downarrow$ & $\uparrow$ & $\uparrow$ \\
\hline \multicolumn{5}{|c|}{ NAFLD Characterization } \\
\hline Liver:Tibia Ratio & $\uparrow$ & $\downarrow \downarrow$ & 个个个 & $\downarrow \downarrow$ \\
\hline ALT & $\uparrow$ & $\uparrow$ & 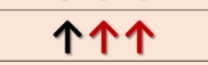 & $\downarrow \downarrow$ \\
\hline AST & - & - & - & - \\
\hline Steatosis & 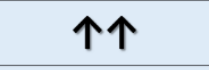 & $\downarrow$ & 个个个 & $\downarrow \downarrow$ \\
\hline Oil Red O & $\uparrow$ & $\downarrow$ & 个个个 & $\downarrow \downarrow$ \\
\hline Hepatic Triglycerides & 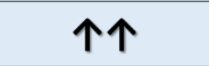 & $\downarrow$ & 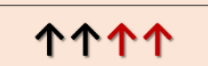 & $\downarrow \downarrow$ \\
\hline
\end{tabular}

Aim 3. Determine the role of sex in cadmium-enhanced, high fat diet-induced NAFLD

Background

Traditionally, the importance of the basic biological differences between males and females has been overlooked in biomedical science (Arnold, 2010; Morrow, 2015). However, more recently it has become abundantly clear that the basic physiological differences between males and females contributes not only to 
disease susceptibility and progression, but also implies there are sex-specific factors that provide disease protection (Arnold, 2010). Starting in 1990 with the establishment of Office of Research on Women's Health (ORWH), the U.S. National Institutes of Health $(\mathrm{NIH})$ has recognized the essentiality of evaluating the effects of sex differences in understanding diseases and for developing more targeted therapies (Clayton and Collins, 2014). Indeed, since 2014 when NIH announced policies requiring grant applicants to assess sex differences in preclinical trials (i.e. in cells and animals) unless inclusion of a single sex is warranted, the number of publications on sex differences has increased (Clayton and Collins, 2014; Lonardo et al., 2019), providing valuable insight for research scientists and clinicians alike.

NAFLD is one of many diseases that are now being considered "sexually dimorphic". Epidemiologically, NAFLD occurs more often in men than in women, and with greater severity (Lazo et al., 2013; Long et al., 2018; Lonardo et al., 2019). In animal models, these differences are generally recapitulated with male mice presenting with more severe steatosis, steatohepatitis, inflammation and tumors in their liver (Kirsch et al., 2013; Ganz et al., 2014; Northeim et al., 2017). Obesity, one of the greatest risk factors for NAFLD, is also a sexually dimorphic disease. For example, in both humans and rodents, females are protected from the adverse consequences of obesity and its associated metabolic pathologies (Pettersson et al., 2012; Palmer and Clegg, 2015). There are however contradictory studies showing female mice are more susceptible to NAFLD despite male mice gaining more absolute weight (Spruss et al., 2012). 
Interestingly, independent of obesity, NAFLD occurs in approximately $20 \%$ of the lean population, indicating the influence of other factors, such as environmental exposures (Younossi et al., 2018). Taken together, it is clear that males are generally more susceptible to NAFLD than females; however, these studies neither take into account the influence of environmental factors, such as exposures to heavy metals, in causing sex-dependent differences in disease outcome nor do they incorporate the interaction of environmental factors and diet.

Exposure to the non-essential heavy metal cadmium is one possible environmental factor that could interact with biological forces to influence the sexual dimorphism of disease outcome. Overall, the health related effects of cadmium are reported to be more common among women (Wang et al., 2003; Vahter et al., 2007; Akesson et al., 2005); however, these reports are generally from occupational exposures and without consideration of low-dose environmental exposures. In addition, these reports do not take into account the effects of early life exposure to cadmium even though there is clear evidence for sex-specific associations between maternal cadmium levels and adverse birth outcomes (Young and Cai, 2020). Further, few studies have investigated the impact of early life-exposure to cadmium on the development of diseases later in the life of females.

Therefore, using the same experimental exposure paradigm presented in Aim 1, we determined the impact of whole life (in utero through adult-hood) exposure to low concentrations of cadmium on the development of high fat dietinduced NAFLD in female mice (Figure 3.25). 


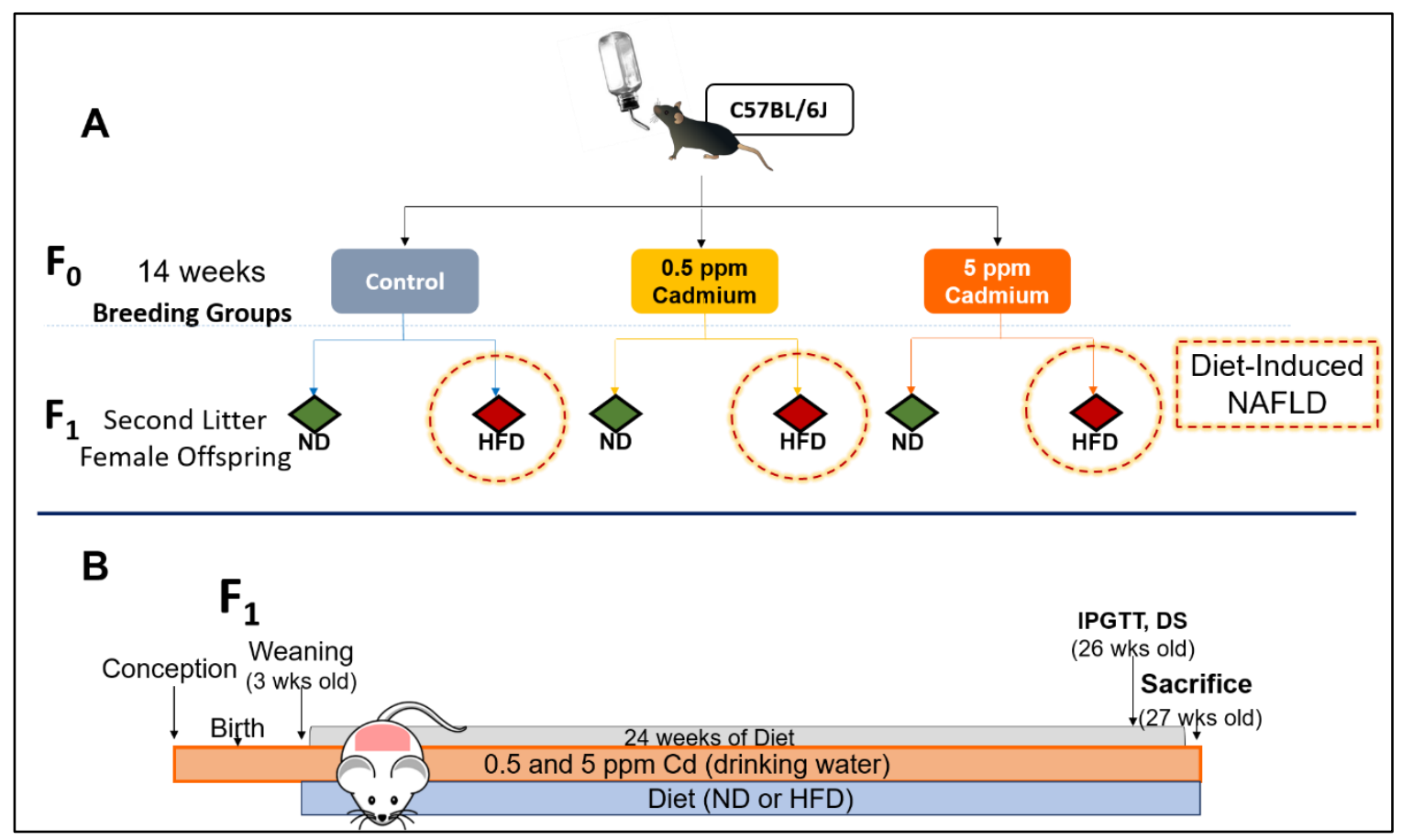

Figure 3.25. Model of whole life exposure of female mice to cadmium and high fat diet. (A) Adult male and female C57BL/6J mice on defined diets were exposed to control drinking water, or water containing 0.5 or $5 \mathrm{ppm}$ cadmium for 14 weeks before being established into breeding pairs. Pregnant dams and female offspring were continuously exposed and continued on the same drinking water regime as their parents after weaning. At weaning, female offspring were also fed either a normal or high-fat diet (ND or HFD, respectively) for 24 weeks. (B) One week prior to sacrifice, IPGTT tests were performed and body composition determine by DEXAscan (DS). IPGTT = intra-peritoneal glucose tolerance test, wks $=$ weeks. 


\section{Findings}

\section{Characterization of the high fat diet model used to induce NAFLD}

Our first objective was to confirm our high fat diet model was successful in female mice. Similar to Aim 1, we began evaluating our model by using four measures: Body weight, DEXAscan, intraperitoneal glucose tolerance tests (IPGTT) and plasms insulin. Female mice fed high fat diet gained significantly more bodyweight over time compared to normal diet fed mice $(\mathrm{p}<0.001)$ (Figure 3.26AC). Although not statistically significant, high fat diet feeding trended to increase percent fat $(p=0.0533)$ (Figure 3.26D), as measured by DEXAscan but did not alter lean tissue mass, bone mineral density or bone mineral content (Figure 3.26E, F and G). Blood glucose levels were significantly increased in high fat dietfed mice (Figure 3.27A and B); however, plasma insulin levels did not change (Figure 3.27C).

Next we determined if our high fat diet induced NAFLD in female mice with five measures: liver weight to tibia length ratios, plasma ALT and AST, H\&E, oil red $\mathrm{O}$ and hepatic triglycerides. High fat diet consumption did not increase liver size (liver weight: tibia length ratios, Figure 3.28A) or plasma transaminases (ALT and AST, Figures 3.28B and C). Histological analysis by H\&E suggested consumption of high fat diet tended to increase steatosis, however, these results were not statistically significant $(p=0.0537)(H \& E$, Figure 3.28D and $E)$. However, high fat diet consumption did not alter oil red O-staining for neutral lipids in the liver. (Figure 3.28F and G). In contrast, biochemical analysis indicated significant increases in triglycerides in high fat diet-fed females compared to female mice fed 


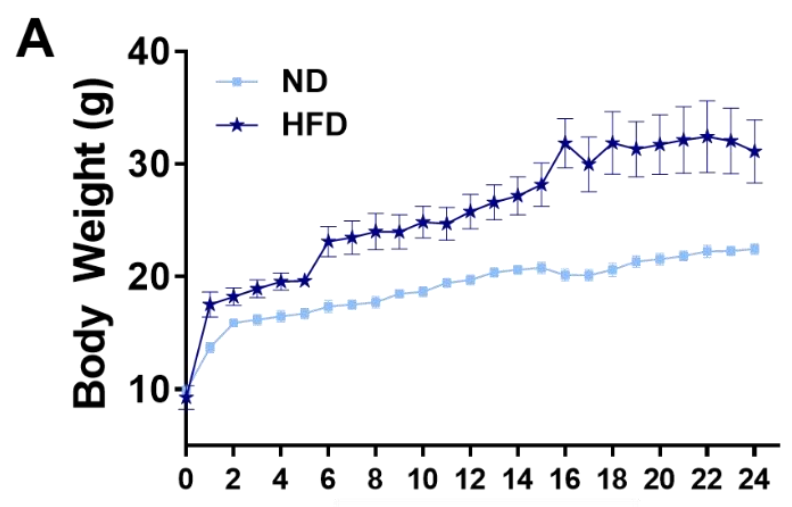

Weeks on Diet
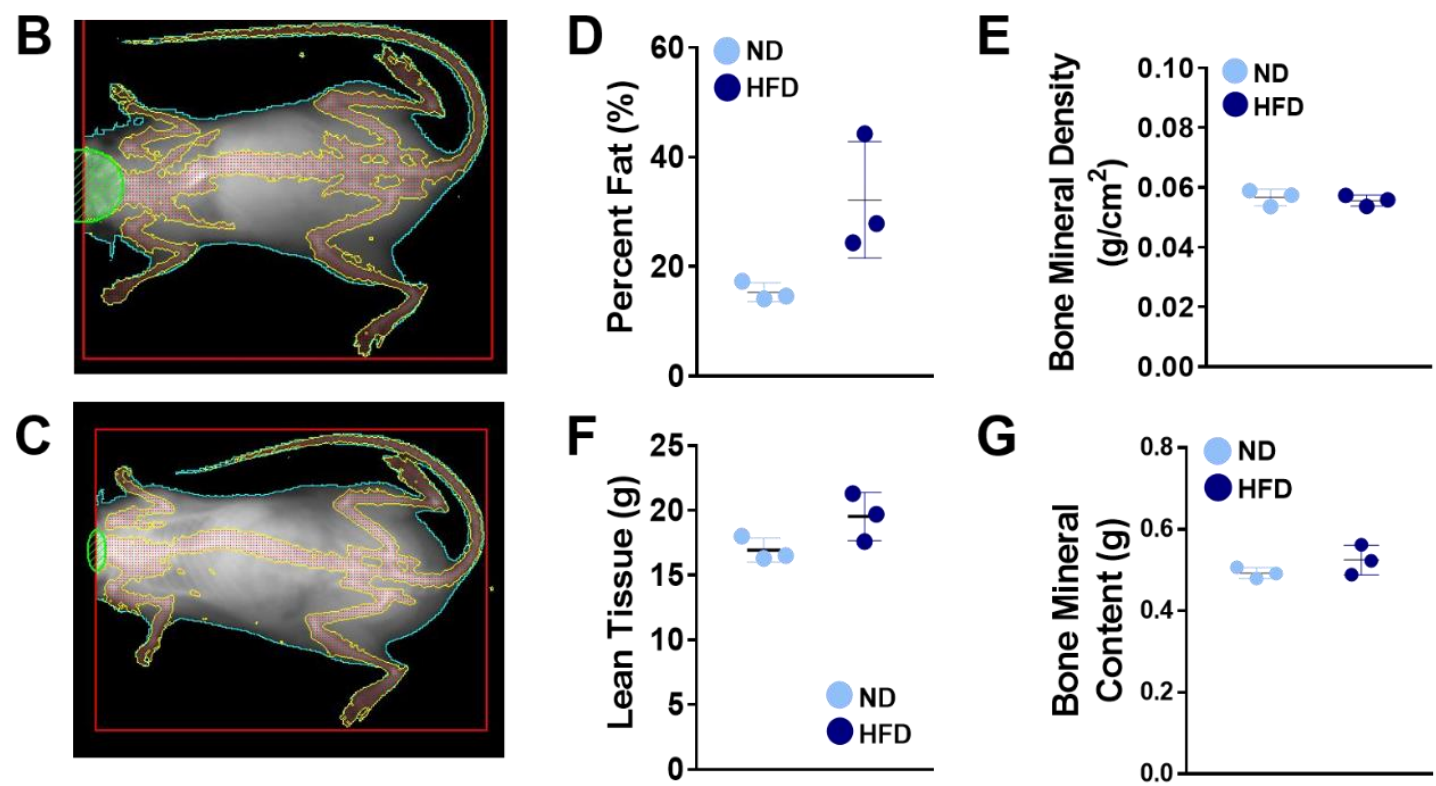

G

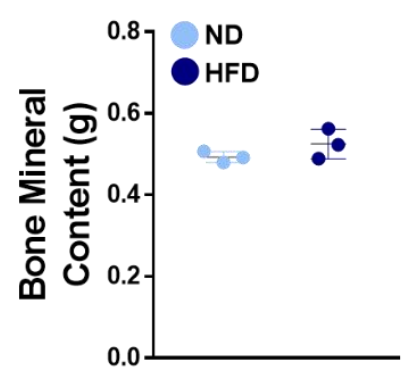

Figure 3.26. Effects of high fat diet on body weight and composition in female mice. This figure shows consumption of high fat diet for 24 weeks post weaning (A) increased body weight gain over time and (B: representative DEXAscan photo of a normal diet fed mouse; C: representative DEXAscan photo of a high fat diet fed mouse) changed body composition, increasing (D) percent fat and (E) lean tissue mass (although not statistically significant) but not $(F)$ bone mineral content or $(G)$ bone mineral density. Results are reported as the mean $\pm S D(n=3)$ for DEXAscan and mean \pm SEM $(n=7-8)$ for body weight. @,$p<0.05$ compared to normal diet. 

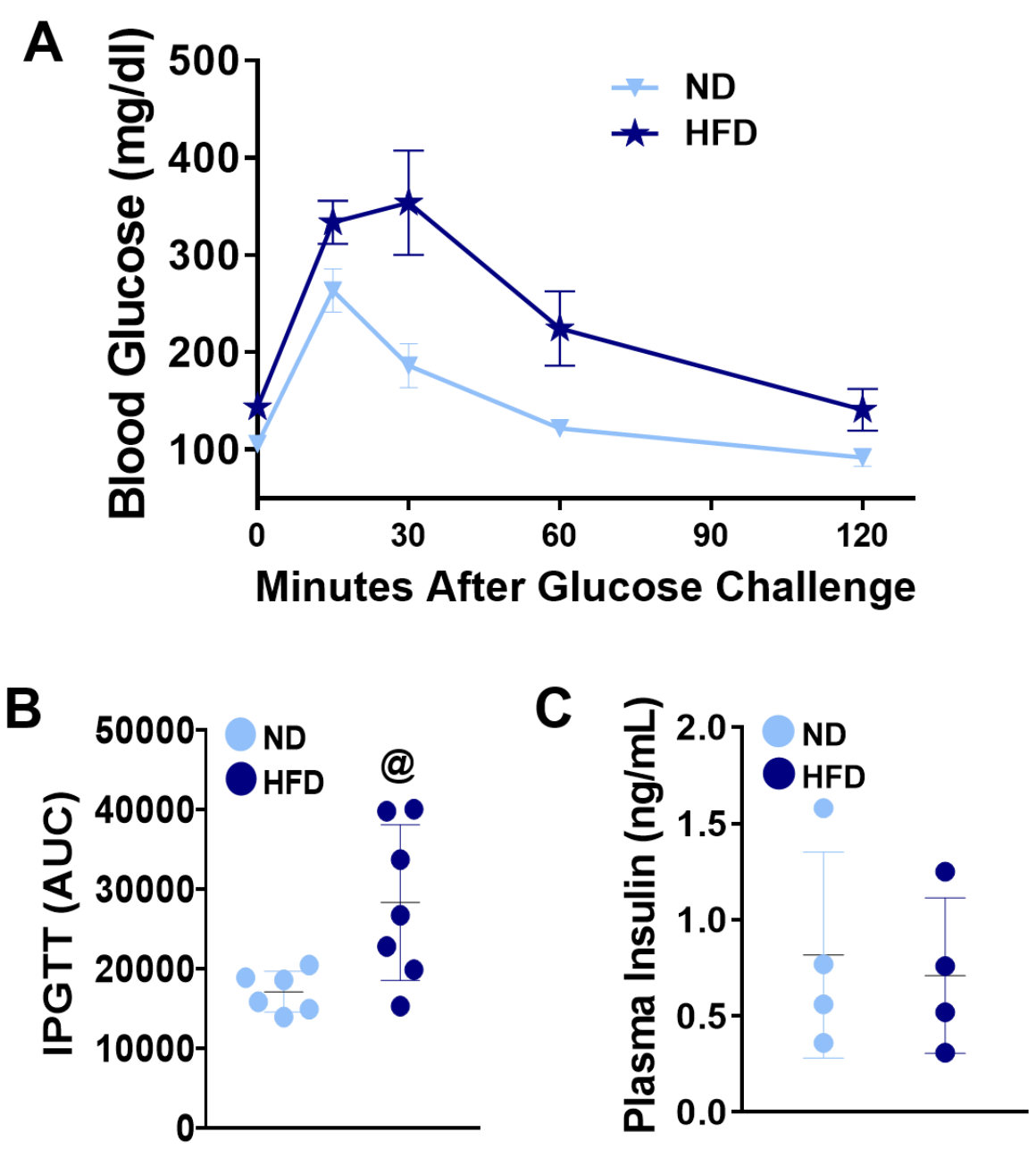

Figure 3.27. Effects of high fat diet on glucose handling and plasma insulin in female mice. This figure shows consumption of high fat diet for 24 weeks post weaning (A) significantly decreased glucose tolerance (indicated by the area under the curve (AUC) from IPGTT), (B) increased blood glucose levels but (C) did not change plasma insulin levels. Results are reported as the mean $\pm S D(n=4-7)$. @, $p<0.05$ compared to normal diet. 

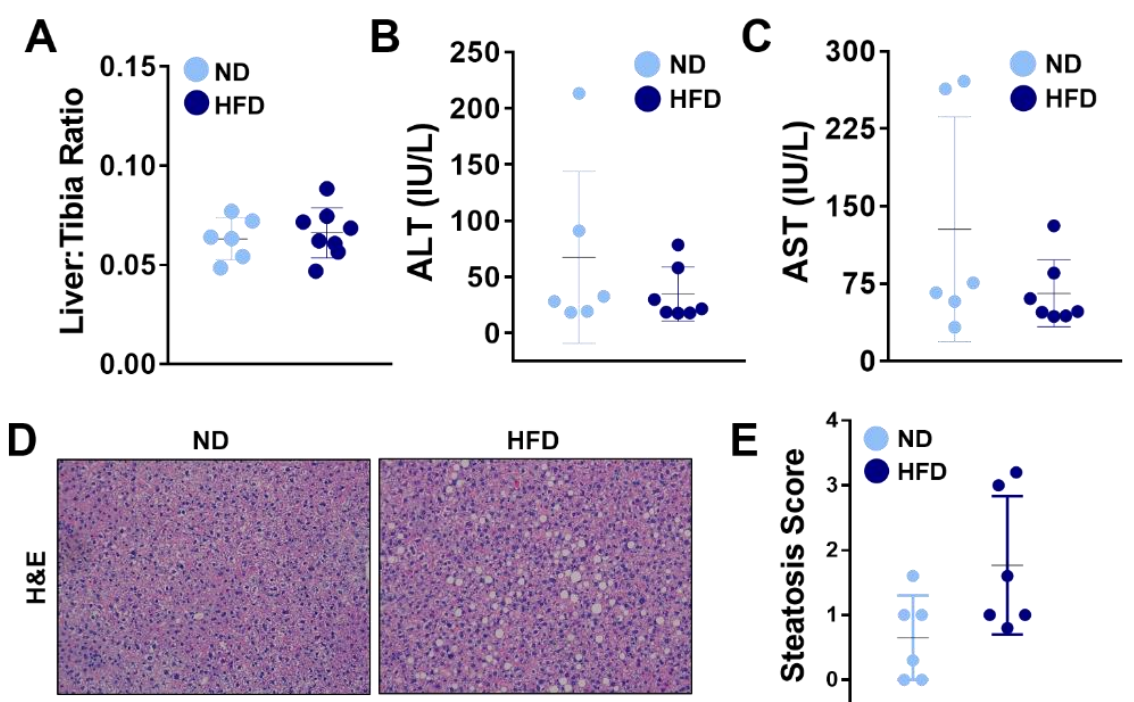

E
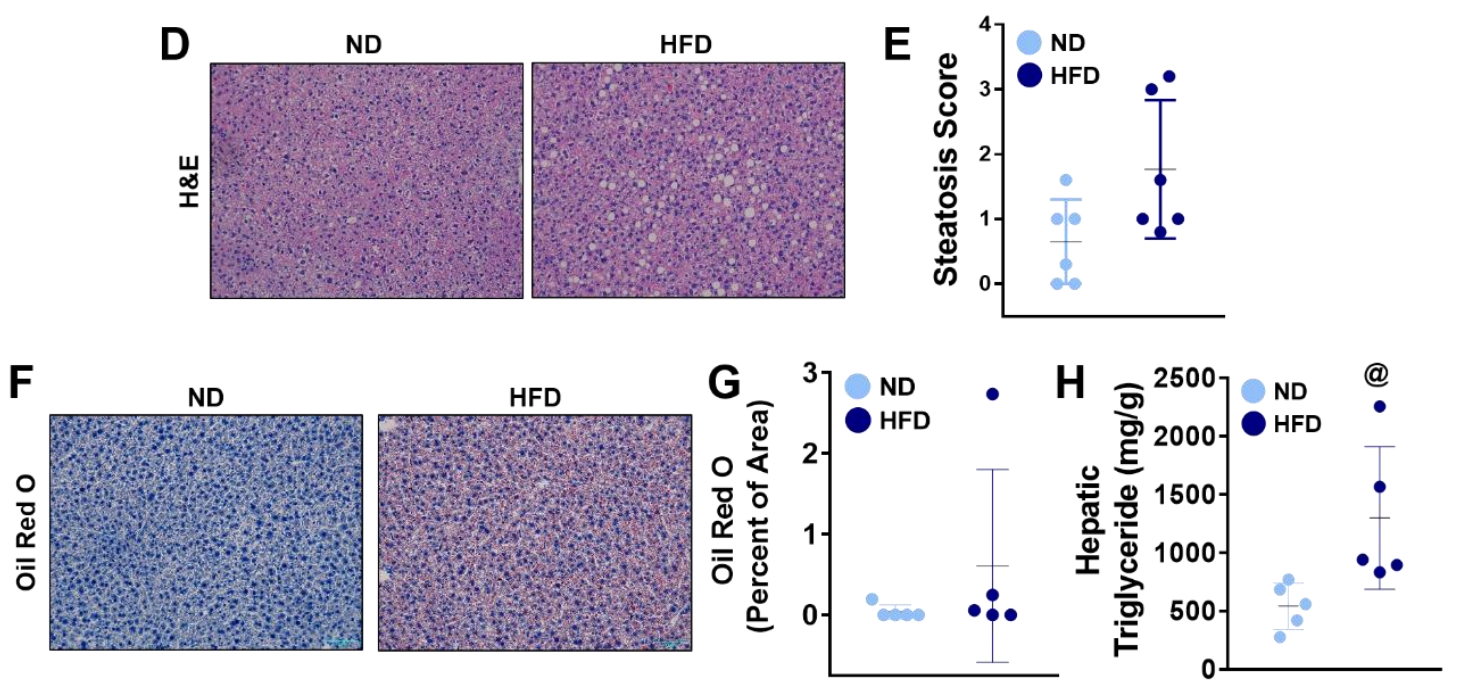

Figure 3.28. Effects of high fat diet on liver injury and NAFLD in female mice.

This figure shows consumption of high fat diet for 24 weeks did not cause liver injury in female mice as indicated by (A) liver: tibia ratios, $(B)$ plasma ALT or $(C)$ plasma AST. However, high fat diet did induce NAFLD. (D) Representative photomicrographs of paraffin embedded liver tissue stained with hematoxylin \& eosin staining (H\&E, 200x). (E) Steatosis was scored as percent of liver cells in 5, 10x fields per liver containing fat. (F) Representative photomicrographs of Oil Red O (neutral lipids, $\times 200$ ) stained frozen liver sections. (G) Quantitative image analysis of ORO-positive staining performed using Image $\mathrm{J}$ : results are shown as percentage of microscope field. $(\mathrm{H})$ Biochemical quantification of hepatic triglycerides. Results are reported as the mean $\pm S D(n=5-8)$. @, $p<0.05$ compared to normal diet. 
normal diet (Figure 3.28H). Taken together, these results indicate our high fat diet model was successful in inducing weight gain and altering blood glucose levels while the measures of steatosis and hepatic triglycerides suggest high fat diet may be inducing NAFLD.

\section{Characterization of the cadmium exposure model}

Next we set forth to confirm the success of our cadmium exposure model in female mice. Epidemiologically, women generally have greater cadmium retention than men (Vahter et al., 2002; Vahter et al., 2007) and we have previously shown cadmium accumulates in the liver of female mice (Young et al., 2019). As expected, in female mice fed a normal diet, cadmium accumulated in the liver in a concentration-dependent manner. Compared to unexposed mice, hepatic cadmium concentrations were 14- and 320-times greater in mice exposed to 0.5 and 5 ppm cadmium, respectively (Figure 3.29A). Very similar values were seen in mice fed high fat diet with the exception of those exposed to $0.5 \mathrm{ppm}$ cadmium. Compared to normal diet fed mice, hepatic cadmium concentrations in HFD mice exposed to $0.5 \mathrm{ppm}$ cadmium were only about 7 -times great than that of unexposed mice, almost half the cadmium found in the corresponding cohort fed a normal diet (Figure 3.29A).

As female mice were exposed to cadmium in their drinking water, we measured water consumption and calculated the average administered cadmium intake (Figure 3.29B and C). Over the course of the study, 0.5 and $5 \mathrm{ppm}$ cadmium exposed female mice drank an average of $4.89 \pm 1.37$ and $4.51 .13 \pm 0.67 \mathrm{~mL}$ 

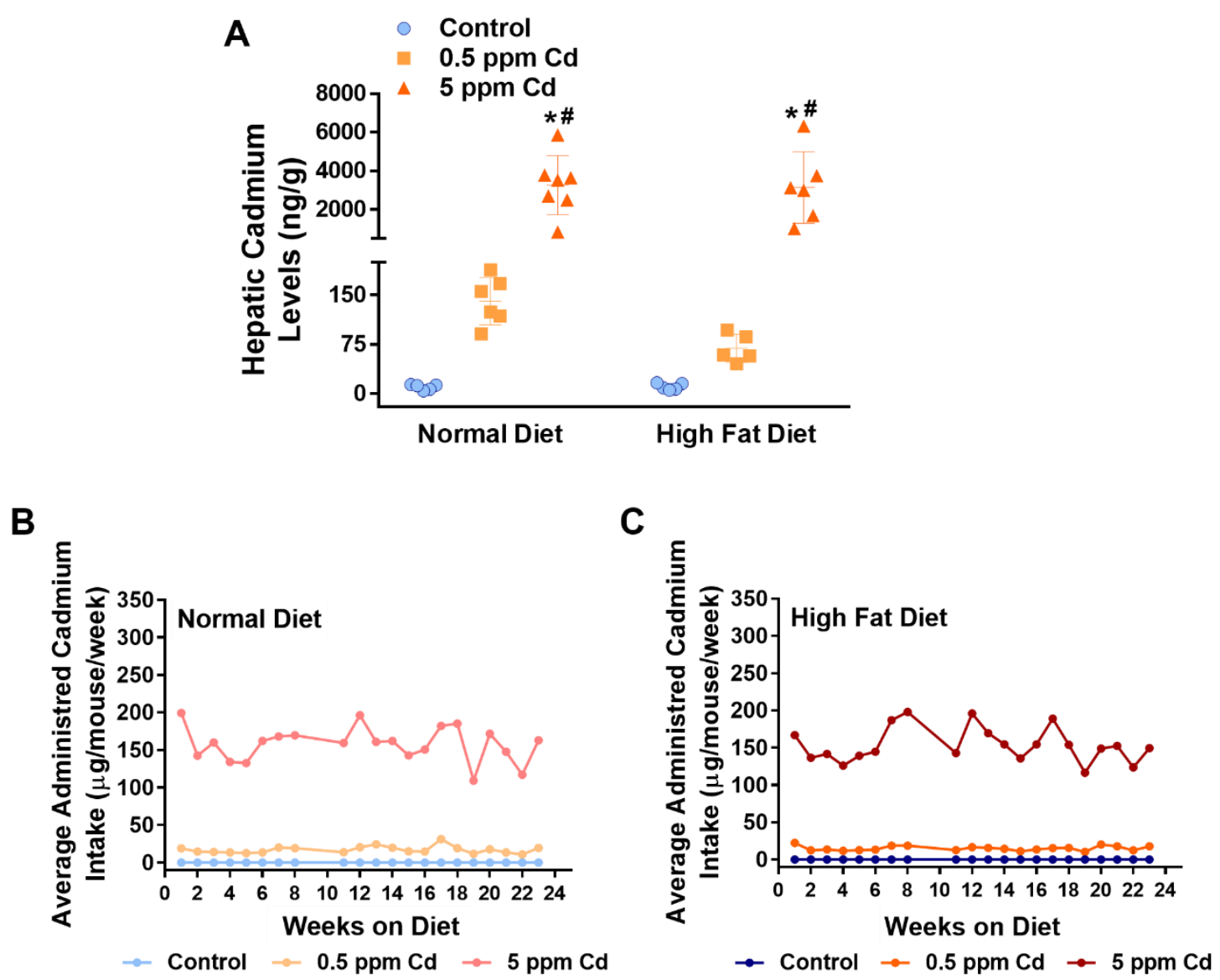

Figure 3.29. Hepatic cadmium levels and weekly administered cadmium intake in female mice. This figure shows cadmium accumulated in the liver in a concentration-dependent manner, reflecting the average administered cadmium intake delivered by drinking water. (A). Hepatic cadmium levels measured by ICPMS. (B) Average weekly administered cadmium intake delivered by drinking water in normal diet fed female mice. (C) Average weekly administered cadmium intake delivered by drinking water in high fat diet fed female mice. ${ }^{*}, p<0.05$ compared to group control; \#, $\mathrm{p}<0.05$ compared to cadmium dose within diet group. 
of water per a day, respectively, compared to controls who drank an average of $4.28 \pm 0.68 \mathrm{~mL}$ of water per a day. Thus, independent of diet, treated female mice drank similar amounts of water compared to controls. The average daily dose of cadmium in female mice that drank water with $0.5 \mathrm{ppm}$ cadmium was $2.45 \pm 0.69$ and $2.15 \pm 0.46 \mu \mathrm{g}$ in normal and high fat diet fed mice, respectively. In mice that drank water with 5 ppm cadmium the average daily dose was significantly greater: $22.56 \pm 3.33(p=<0.0001)$ and $21.95 \pm 3.32 \mu g(p=<0.0001)$ in normal and high fat diet fed mice, respectively.

\section{Impact of cadmium exposure on HFD-fed female mice}

We next investigated the effects of whole life, low dose cadmium exposure on high fat diet-fed female mice using the same nine measures implemented in our model confirmation in male mice (Aim 1).

\section{Body weight and composition}

Exposure to cadmium did not alter body weight gain in normal diet or high fat diet fed female mice (Figure 3.30A and B). In female mice that consumed normal diet, cadmium exposure did not impact any of the parameters measured by DEXAscan. However, there was a diet effect on $\%$ fat and lean tissue mass ( $p$ $=0.0023$ and $p=0.0053$, respectively). Further multiple comparison analysis revealed exposure to $5 \mathrm{ppm}$ cadmium significantly increased \% fat (Figure 3.30C) and lean tissue mass (Figure 3.30D) in high fat diet-fed mice compared to their 
A

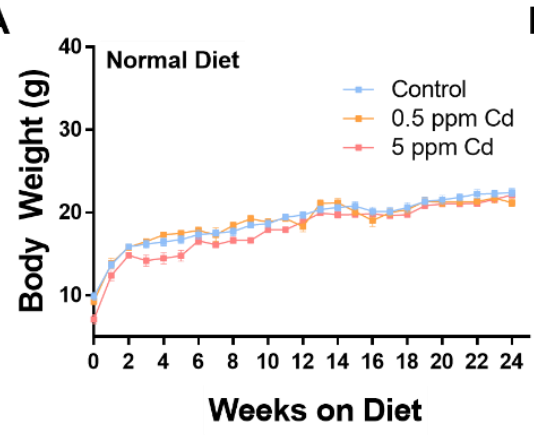

C

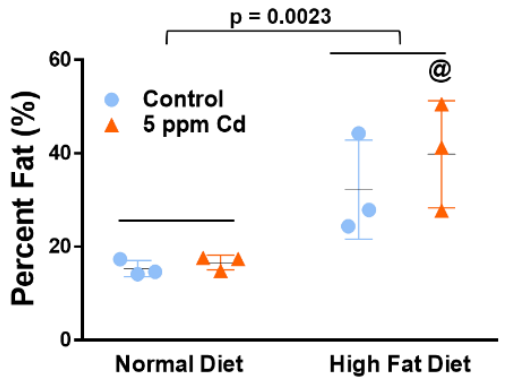

D

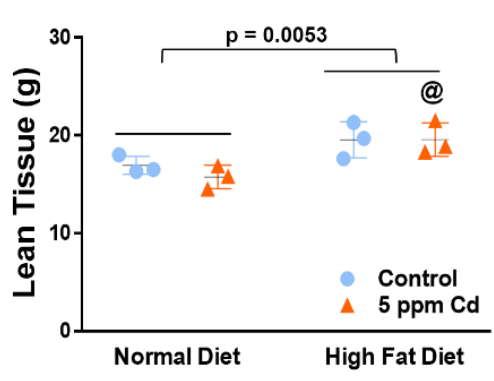

B
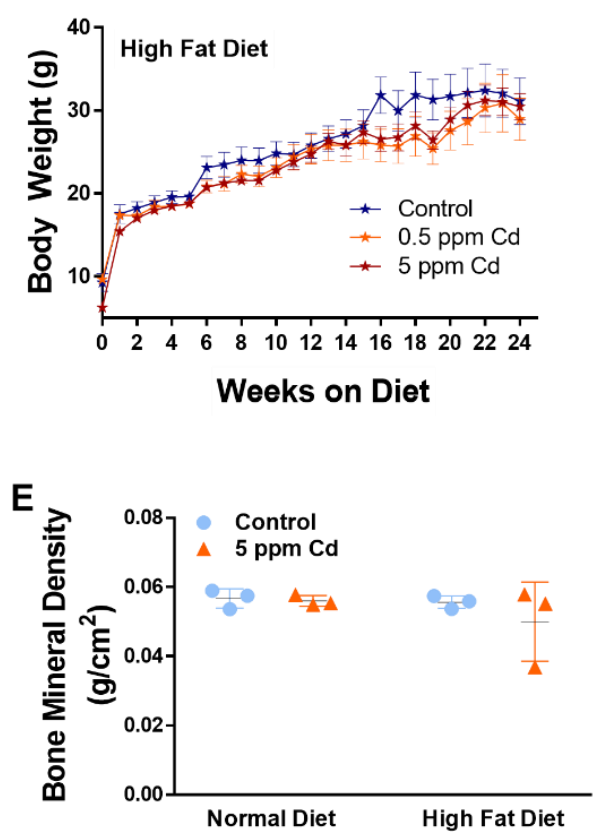

$\mathbf{F}$

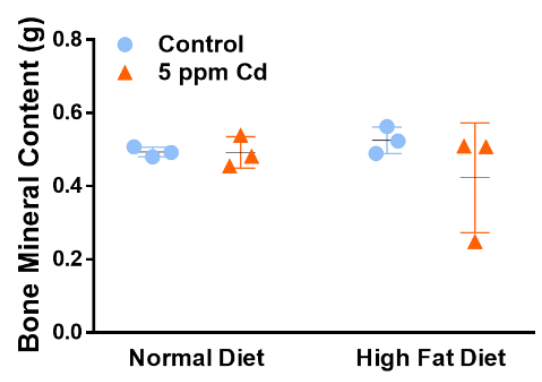

Figure 3.30. Effects of whole life cadmium exposure and high fat diet on body weight and composition in female mice. Whole life exposure to cadmium does not alter body weight gain over time. Body weight was measure once a week for 24 weeks starting at weaning, continuing until sacrifice. Weekly body weight in (A) normal diet fed male mice and (B) high fat diet fed mice. DEXAscan was performed one week prior to sacrifice for control and $5 \mathrm{ppm}$ cadmium exposed mice to assess body composition. (C) Percent fat, (D) lean tissue mass, (E) bone mineral density and (F) bone mineral content. Results are reported as the mean $\pm \operatorname{SD}(n=3)$ for DEXAscan and mean \pm SEM $(n=5-12)$ for body weight. @, p $<0.05$ compared to corresponding normal diet. 
corresponding normal diet-fed cohort, but not bone mineral density (Figure 3.30E) or bone mineral content (Figure 3.30F).

\section{IPGTT and plasma insulin}

In normal diet-fed female mice, cadmium exposure did not alter glucose handling, as measured by IPGTT, or plasma insulin levels (Figure 3.31 A, C and D). In female mice fed high fat diet, exposure to $0.5 \mathrm{ppm}$ cadmium significantly decreased blood sugar clearance compared to the corresponding mice fed normal diet and these levels were similar to that of the high fat diet-fed control mice (Figure 3.31B and C). Interestingly, in mice that were fed high fat diet, exposure to the higher concentration of cadmium (5 ppm) led to quicker blood glucose clearance compared to mice exposed to the lower cadmium concentration ( $0.5 \mathrm{ppm}$ ) (Figure 3.31B and C). Exposure to cadmium did not impact plasma insulin levels in either normal diet or high fat diet-fed mice (Figure 3.31D). Overall, cadmium exposure did not significantly alter blood sugar handling or insulin levels in the plasma, suggesting that cadmium is not significantly involved with insulin resistance in female mice.

Hepatic injury and measures of NAFLD

In our model, diet nor exposure to $5 \mathrm{ppm}$ cadmium altered liver size. However, exposure to $0.5 \mathrm{ppm}$ cadmium increased liver size in high fat diet-fed female mice compared to exposed mice fed normal diet (Figure 3.32A). Independent of which diet mice were fed, cadmium exposure did not change 

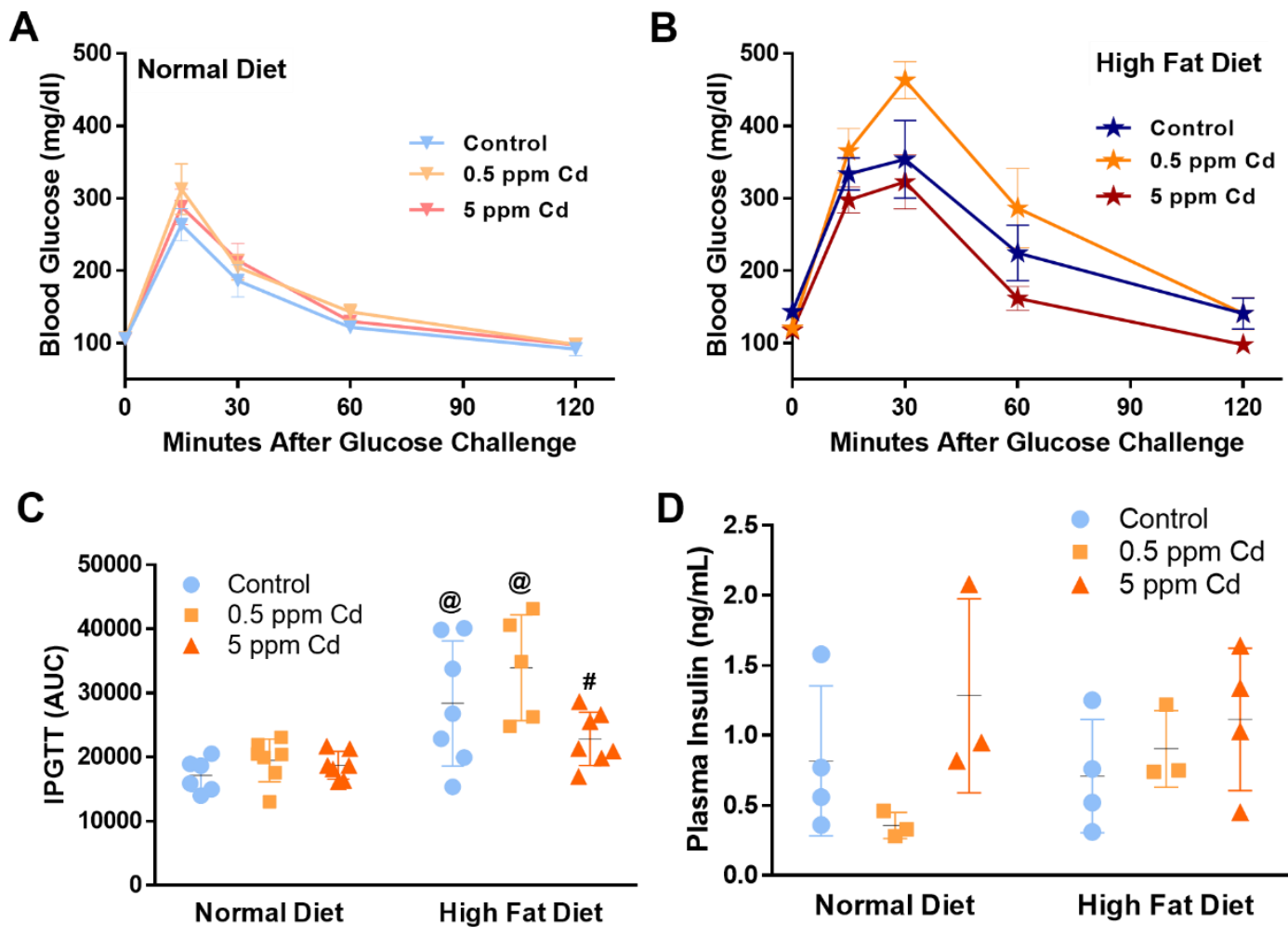

Figure 3.31. Effects of whole life exposure to cadmium and high fat diet on glucose handling and plasma insulin in female mice. Whole life exposure to cadmium does not alter high fat diet-associated changes in glucose handling in female mice. (A) Blood glucose levels in normal diet fed mice and (B) high fat diet fed mice after IPGTT, performed one week prior to sacrifice. (C) Integrated area under the curve (AUC) showing quantitative changes in blood glucose levels after glucose challenge. (D) Insulin levels in plasma at time of sacrifice. Results are reported as the mean $\pm S D(n=3-7)$. \#, $p<0.05$ compared to cadmium dose within diet group; @, p < 0.05 compared to corresponding normal diet. 


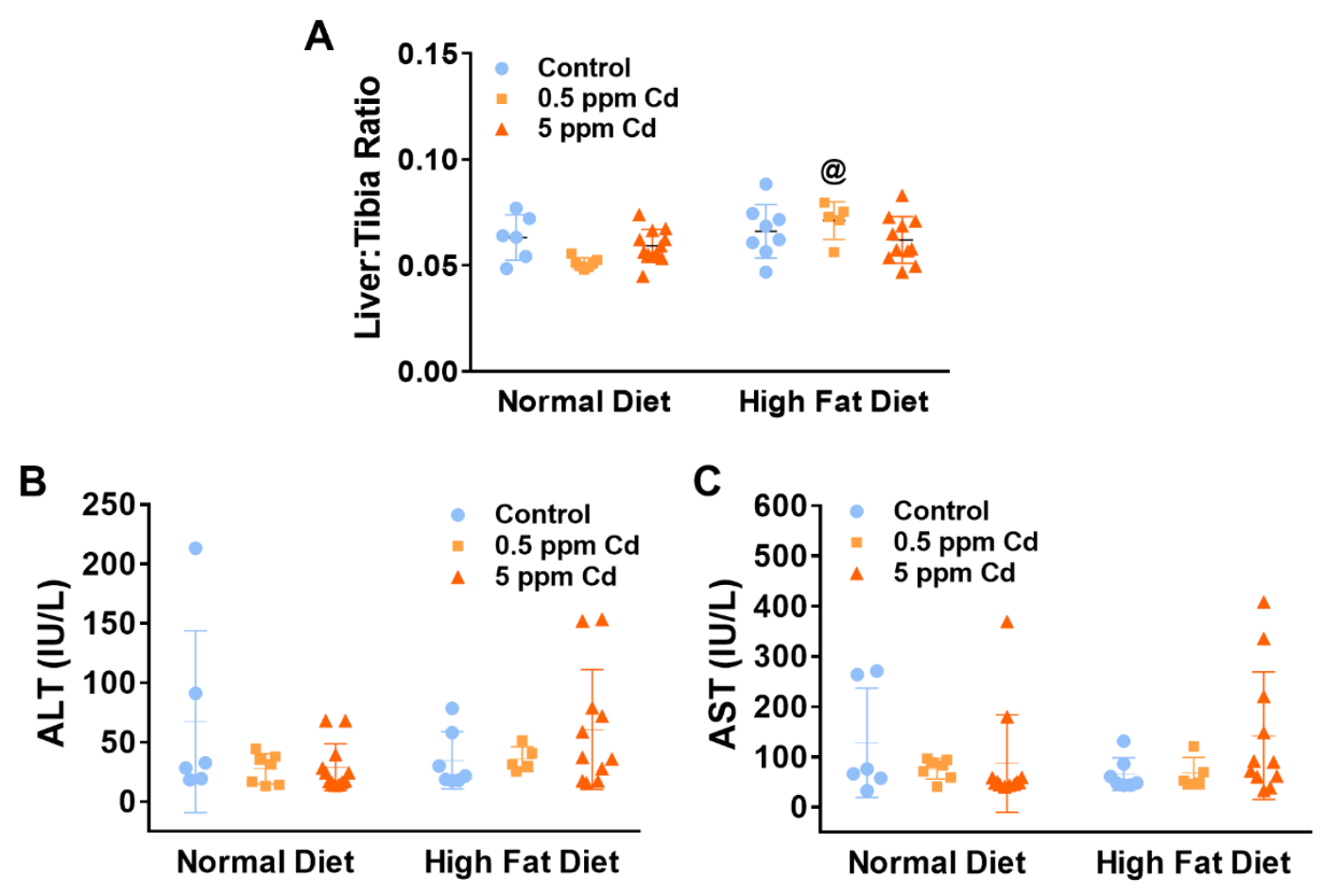

Figure 3.32. Effects of whole life cadmium exposure and high fat diet on liver injury in female mice. This figure shows cadmium exposure does not cause liver injury in female mice as measured by the $(A)$ ratio of liver weight in grams to tibia length in millimeters, (B) plasma aspartate aminotransferase (AST) or (C) plasma alanine aminotransferase (ALT) activity. No statistical significance was observed. Results are reported as the mean \pm SD $(n=6-12)$. @, $p<0.05$ compared to corresponding normal diet. 
A
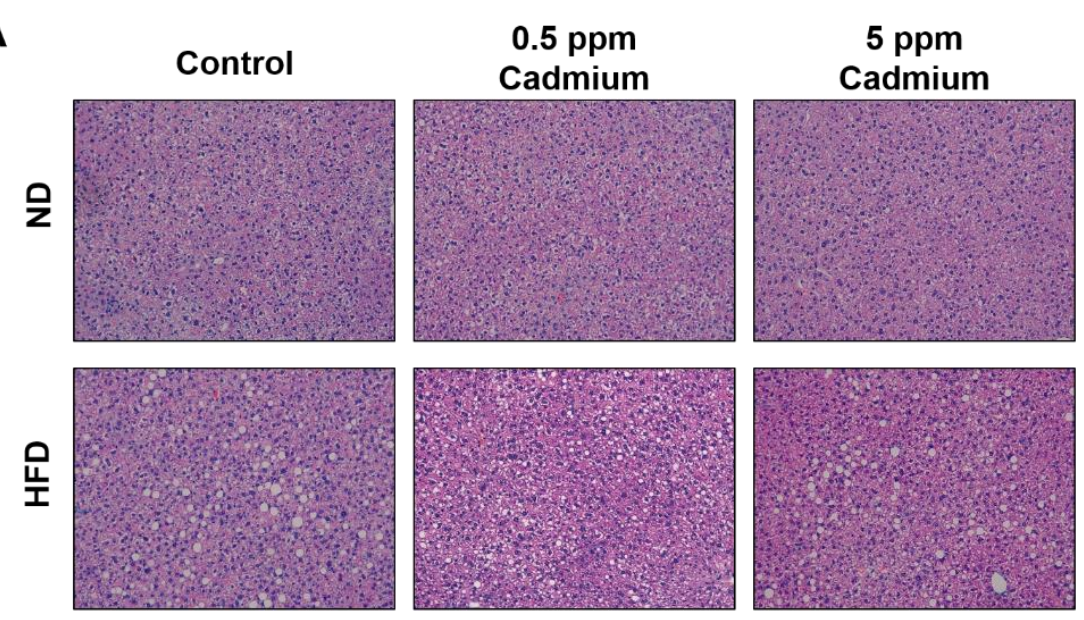

B
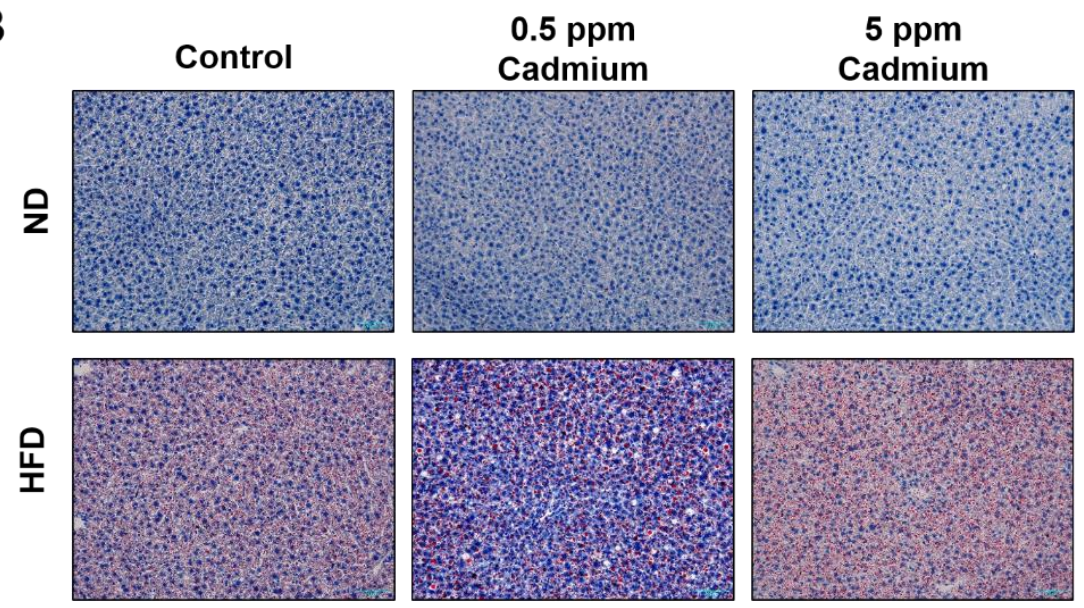

C

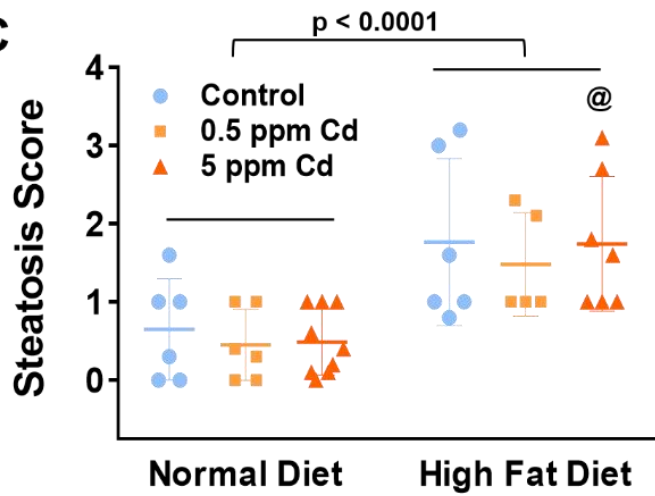

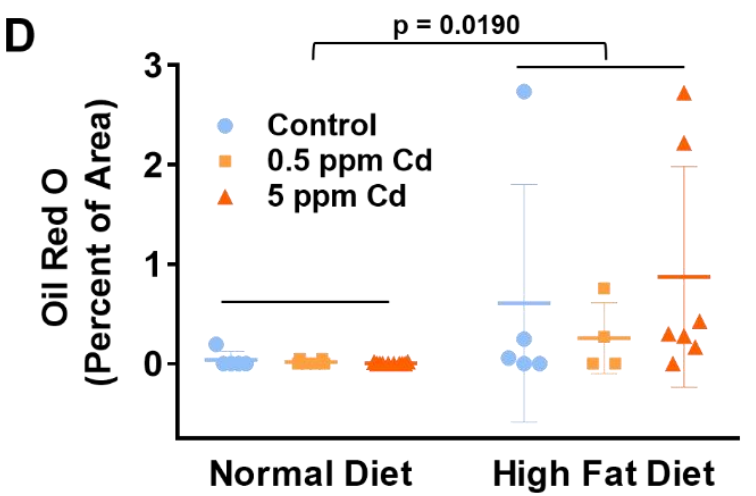

Figure 3.33. Effects of whole life cadmium exposure and high fat diet on NAFLD in female mice. This figure shows exposure to cadmium influences high fat diet-induced steatosis. (A) Representative photomicrographs of paraffin 
embedded liver tissue stained with hematoxylin \& eosin staining (H\&E, 200x). (C) Steatosis was scored as percent of liver cells in 5, 10x fields per liver containing fat. (B) Representative photomicrographs of Oil Red O (neutral lipids, $\times 200$ ) stained frozen liver sections. (D) Image analysis of ORO-positive staining was performed using Image $\mathrm{J}$, and results are shown as percentage of microscope field. Results are reported as the mean $\pm S D(n=4-10)$. @ , $p<0.05$ compared to corresponding normal diet. 
A
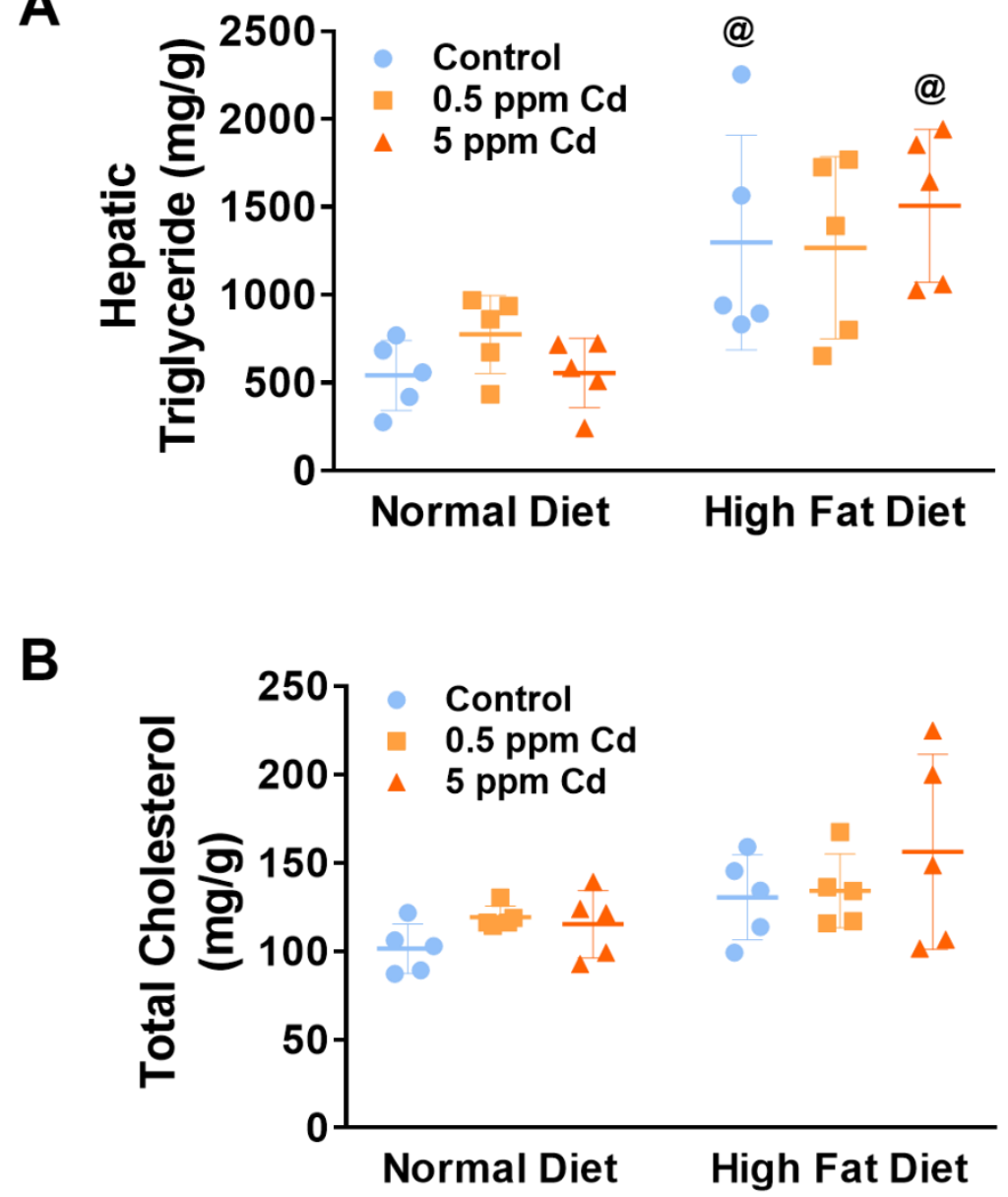

Figure 3.34. Effects of whole life cadmium exposure and high fat diet on biochemical measures of NAFLD in female mice. This figure shows cadmium exposure does not alter hepatic triglycerides or total cholesterol in in high fat diet fed female mice. (A) Hepatic triglyceride levels. (B) Total hepatic cholesterol levels. Results are reported as the mean \pm SD $(n=4-6)$. @, $p<0.05$ compared to corresponding normal diet. 
plasma transaminases (Figure 3.32B and C), indicating exposure to cadmium did not induce liver injury in female mice. Histological assessment of hepatic structure (Figure 3.33A and C) and neutral lipid staining (Figure 3.33B and D) showed a diet effect on steatosis $(p<0.0001)$ and on lipid accumulation $(p=0.0190)$, independent of Similarly, independent of diet, histological assessment showed cadmium exposure. did not change hepatic structure (Figure 3.33A and $\mathrm{C}$ ) or alter lipid accumulation in the liver (Figure 3.33B and D). These results were further supported by biochemical analysis of hepatic triglycerides (Figure 3.34A) and total hepatic cholesterol (Figure 3.34B), of which exposure to cadmium did not influence. Taken together these data suggest cadmium exposure in female mice did not cause liver injury. Furthermore, although consumption of a high fat diet may induce steatosis, cadmium exposure did not alter the diet-induced hepatic outcomes. In male mice, we saw that supplementing the diet with zinc attenuated high fat diet-induced, cadmium-exacerbated NAFLD. Therefore we looked at the hepatic zinc levels in female mice. Interestingly, in female mice fed high fat diet, exposure to 5 ppm cadmium increased zinc levels by $13 \%$ compared to controls and $16 \%$ compared to corresponding normal diet fed mice (Figure 3.35). Overall, the increase in zinc levels may provide protection against cadmium-induced injury in high fat diet fed female mice, as was seen in male mice supplemented with zinc. 


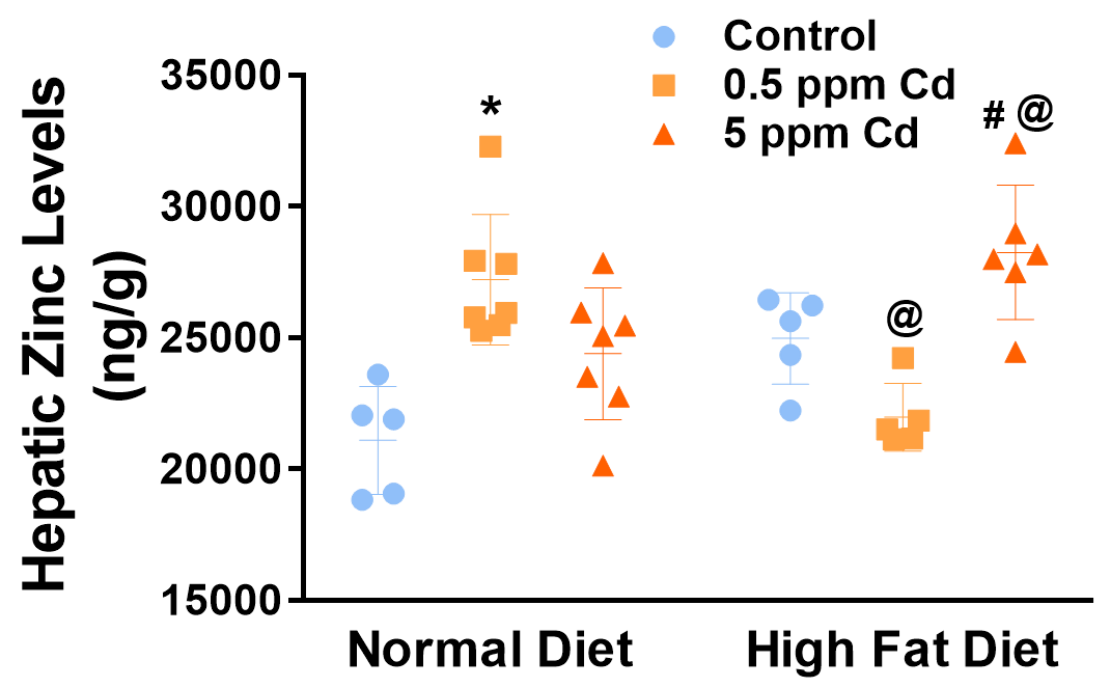

Figure 3.35. Hepatic zinc levels in female mice. This figure shows hepatic zinc levels in female mice after 24 weeks of diet, post weaning, measured by ICP-MS. Results are reported as the mean $\pm S D(n=5-7) .{ }^{*}, p<0.05$ compared to group control; \#, p $<0.05$ compared to cadmium dose within diet group; @, $\mathrm{p}<0.05$ compared to corresponding normal diet. 


\section{Summary}

In this aim we showed feeding female mice high fat diet for 24 weeks increased bodyweight gain overtime and induced hyperglycemia without changing plasma insulin levels. Although high fat diet did not cause hepatomegaly or increase plasma transaminases, histological analysis revealed high fat diet did cause steatosis and significantly increased hepatic triglycerides. Taken together, these results suggest high fat diet feeding in female mice may induce NAFLD, although the severity of the diseases appears to be much less compared to the male mice studied in Aim 1. Our data also show that while female mice accumulated cadmium in their livers, cadmium exposure did not act as a second hit and did not exacerbate high fat diet-altered measure of fatty liver in female mice as it did in the male mice nor did exposure to cadmium alone (in normal diet fed mice) influence any of the measures investigated. 


\section{CHAPTER 4: DISCUSSION}

\section{Overview}

NAFLD is a major global public health concern accounting for more than $\$ 103$ billion dollars in annual medical costs in the United States alone (Younossi et al 2016). The prevalence of NAFLD is rapidly growing in parallel with the epidemic of obesity with up to $91 \%$ of obese patient's developing liver disease (Machado et al 2006). Interestingly, in the absence of obesity, $10-20 \%$ of the population still develop NAFLD, suggesting the contribution of other risk factors (Younossi et al 2018). In fact, NAFLD is a multifactorial disease in which the pathogenesis is more accurately explained by multiple insults acting together.

Sex is a contributing factor as males are more likely to suffer from NAFLD than females (Hyder et al 2013). Environmental exposure to the non-essential metal cadmium may be a third contributing factor as it has been associated with the development NAFLD in both epidemiology and animal studies (Hyder et al 2013, Go et al 2015; Ba et al 2017). However, the interaction of cadmium exposure and consumption of a high fat diet and sex are three interactive factors in NAFLD pathogenesis that remained unexplored until this study. We found diet, sex and environmental exposure interact to cause NAFLD. Notably, we also found major differences in outcome based on cadmium level. Accordingly, below we discuss first the outcomes with $5 \mathrm{ppm}$ cadmium and then with $0.5 \mathrm{ppm}$ cadmium. 
Exposure to $5 \mathrm{ppm}$ cadmium exacerbates high fat diet-induced NAFLD in male mice

Sex, Cadmium, High Fat Diet and NAFLD

NAFLD is a sexually dimorphic disease occurring more often and with greater severity in men than in women (Ballestri et al., 2017; Lonardo et al., 2019). In general, animal models recapitulate the sex differences observed in human populations with NAFLD (Ganz et al., 2014; Norheim et al., 2017). We found 5 ppm cadmium exposure exacerbated high fat diet-induced weight gain, insulin resistance, hepatic injury (hepatomegaly and plasma transaminase), steatosis and lipid deposition in male mice and although this exposure causes greater cadmium accumulation in the liver of females, it does not impact high fat diet-induced NAFLD in female mice. We are the first to report these outcomes supporting our hypothesis that cadmium exposure exacerbates high fat diet-induced NAFLD in males more than females and supporting the multiple hit hypothesis that NAFLD prevalence and progression is influenced by multiple variables.

The mechanisms by which cadmium exacerbates high fat diet-induced NAFLD in male mice are uncertain and beyond the scope of this model-generating study. Nevertheless, our data do offer some insights into possible mechanistic directions. Data indicate a high fat diet alone can contribute to NAFLD by causing an imbalance between lipid availability (lipogenesis/de novo lipogenesis) and lipid removal (lipolysis and fatty acid $\beta$-oxidation) leading to excessive accumulation of neutral lipids in the liver (Koo, 2013; Saponaro et al., 2015; Ipsen et al., 2018). For example, Oosterveer et al (2009) showed induction of hepatic fatty acid synthesis 
in C57BL/6J male mice fed high fat diet for 6 weeks. However, other studies have shown high fat diet supersession of de novo lipogenesis (Duarte et al., 2014). In two separate studies in Wistar rats fed either a $42 \%$ or a $51.8 \%$ fat diet for 6 and 12 weeks, respectively, fatty acid $\beta$-oxidation levels were decreased (Lionetti et al., 2014; Aoun et al., 2012). In contrast, consumption of both 55 and $60 \%$ high fat diet in rodents is associated with increased fatty acid $\beta$-oxidation activity (Cardoso et al., 2013; Satapati et al 2012). Taken together, the contradictions in the literature exemplified the complexities of lipid metabolism. Therefore, if cadmium were to disrupt one or both of these pathways, this may cause the exacerbated outcome we observed.

In our study we considered cadmium disruption of sterol regulatory elementbinding protein (SREBP-1)-mediated lipogenesis as a possible mechanisms for the cadmium exacerbation of high fat diet-induced NAFLD as SREBP-1 plays a major role in NAFLD pathogenesis and cadmium exposure is associated with upregulation of genes associated with hepatic lipid metabolism (Ferre and Foufelle, 2007; He et al., 2015; Moslehi and Hamidi-zad, 2018; Zhang et al., 2018). SREBP-1 increases the rate of fatty acid synthesis though transcriptional activation of lipogenic enzymes such as fatty acid synthase (FASN) and stearoyl coenzymeA desaturase1 (SCD1) (Fon Tacer and Rozman, 2011), both of which are found to have increased expression in NAFLD patients (Kohjima et al., 2007; Kotronen et al., 2009). In animal studies, overexpression of SREBP-1 resulted in increased lipogenesis and fatty liver (Shimano et al 1997) while inactivation of the SREBP-1 
gene in an obese mouse model reduced total triglycerides by half (Park et al., 2014).

However, in our model, consumption of high fat diet with or without cadmium exposure did not trigger a SREBP-1 mediated increases in lipid synthesis in male mice, but actually a decrease in its downstream targets FASN and SCD-1, indicating increased de novo lipogenesis is likely not a factor. As it relates to our results in unexposed mice fed a high fat diet, this outcome is consistent with some of the literature showing high fat diet actually suppresses de novo lipogenesis (Duarte et al., 2014). The literature surrounding cadmium exposure and SREBP-1 is sparse and data associating cadmium with upregulation of hepatic lipid metabolism genes was obtained at cadmium concentrations much greater than was used in our model (2- and 20-fold higher compared to our highest exposure level) (Zhang et al., 2018).

Another mechanism by which cadmium may exacerbate high fat dietinduced NAFLD is through disruption of $\beta$-oxidation. However, we did not consider this pathway in our model. Data concerning cadmium interacting with fatty acid $\beta$ oxidation are very limited. In Chinese toads (Bufo gargarizans) exposure to low levels of cadmium did not affect fatty acid $\beta$-oxidation, whereas exposure to higher levels of cadmium resulted in reduced fatty acid $\beta$-oxidation (Wu et al., 2017). In another recent study, environmental cadmium exposure for 20 weeks inhibited fatty acid oxidation, possibly through the sirtuin 1 (SIRT1) pathway (He et al., 2019). Given the absence of effect on lipogenesis, this pathway will be an important future consideration for our model. 
In contrast to the lipogenesis data, we did find a strong effect on metallothionein. The interactions of cadmium with high fat diet we found in male mice are consistent with studies showing cadmium interacts with a western high fat diet to disrupt the general dysregulation of essential metal homeostasis associated with obesity (Garcia et al., 2009; Young et al., 2019). Metallothionein plays a major role in the homeostatic regulation of essential metals (Sakulsak, 2012) and animal studies have shown metallothionein has the potential to prevent obesity and obesity related disease (Sato et al., 2013; Byun et al., 2011; Lindeque et al., 2015; Kawakami et al., 2019). In fact, metallothionein knockout mouse strains are more susceptible to high fat diet-induced effect (Lindeque et al., 2015; Gu et al., 2107). Taken together it is likely that cadmium and high fat diet interact to reduce metallothionein induction, resulting in increased susceptibility to both cadmium and high fat diet insult.

Indeed, we also found high fat diet suppresses metallothionein induction in the presence of cadmium. Our data show, in mice fed normal diet, $5 \mathrm{ppm}$ cadmium increased mRNA and protein levels of hepatic metallothionein. However, in high fat diet-fed mice, 5 ppm cadmium exposure dramatically lowered metallothionein response compared to normal diet-fed mice, indicating a possible interaction between high fat diet and cadmium that hinders the protective response of metallothionein, suggesting a possible mechanism by which exposure to cadmium may exacerbate high fat diet-induced liver disease.

The sexually-dimorphic outcome of our results may be explained by differential regulation of zinc homeostasis in males and females. For example, high 
serum zinc levels are associated with increased risk of developing metabolic syndrome in men, while similar zinc levels appear to be protective in women (Ghasemi et al., 2014). Additionally, studies have shown differential expression of zinc transporters (Foster et al., 2011) in men and women. In our study we show hepatic zinc levels were unchanged in male mice regardless of diet or cadmium exposure. However, in female mice fed high fat diet, exposure to $5 \mathrm{ppm}$ cadmium significantly increased hepatic zinc levels. In fact, these levels were similar to those reported in the male mice in our study after zinc supplementation. The importance of zinc status in our model will be discussed in detail below. Another explanation or our sexually-dimorphic results are hormones, in particular estrogen. However, we have yet to explore this in our model. Both epidemiological and experimental studies highlight the protective role of estrogens against metabolic diseases, including NAFLD, at least before menopause (Lonardo et al., 2019; Tramunt et al., 2020).

Furthermore, in models of high fat diet-induced obesity, estrogen driven protection is abolished by bilateral ovariectomy and this protection is restored upon administration of estrogen (Handgraaf et al., 2013; Riant et al., 2009). Even in male mice, administration of estrogen even protected male mice from obesity complications (Dakin et al., 2015). In addition, cadmium exhibits both estrogenic and androgenic properties and thus is regarded as a potential endocrine disrupter and may, in part, explain the sex-specific differences of cadmium exposure on diseases outcome, including NAFLD (Vahter et al., 2007; Nasiadek et al., 2018). Taken together, the estrogenic effects of cadmium will be important to consider in 
our model and may help further elucidate the underlying mechanism responsible for the sexual dimorphism in our model.

Sex, Cadmium and NAFLD

Epidemiologically, cadmium burden is associated with liver-related mortalities and NAFLD (Hyder et al., 2013). Thus, we considered the ability of cadmium alone to induce NAFLD. We found exposure to $5 \mathrm{ppm}$ cadmium, starting in utero and continuing for 24 weeks post weaning, did not induce NAFLD, though we did find increased levels of liver transaminases (ALT and AST) in cadmium exposed mice independent of diet.

Our data conflict with the only other previous in vivo report of cadmium and NAFLD, which reported cadmium exposure induces hepatotoxicity and dysregulates NAFLD-related metabolic pathways (Go et al., 2015). That study exposed 8 week-old male mice for 20 weeks to $10 \mathrm{ppm}$ cadmium in drinking water. Consistent with our study, they found both ALT and AST were elevated, but they also found liver triglycerides and lipid deposition were also elevated, which were not found in our study. The difference is most likely due to the fact that the cadmium exposure they used was twice the exposure we used in our study. This possibility would suggest the changes in ALT and AST may precede the changes in lipid deposition and hepatic triglycerides following cadmium exposure. Heavy metals, and cadmium in particular, are associated with elevated ALT and AST activity (Kang et al., 2013; Rao et al., 2017) and these elevations have been found in otherwise healthy adults (Kang et al., 2013). Therefore it is possible that the levels 
of cadmium in both studies was sufficient to increase transaminase levels, but in our study, the lower concentration of cadmium was not sufficient to cause progression to NAFLD.

It is also notable that mice in our study were exposed to cadmium starting in utero whereas in the study by Go et al (2015) mice were not exposed to cadmium until they were 8 weeks old as the authors stated in their methods that they specifically chose 8 week old mice as their intention was not to assess developmental toxicity. Thus, it is possible the chronicity of the exposure may have played a role in these different outcomes. For example, it is well documented that prenatal cadmium exposure is associated with altered DNA methylation patterns (Castillo et al., 2012; Kippler et al., 2013; Mohanty et al., 2015). Castillo et al. (2012) reported altered methylation patterns of the hepatic glucocorticoid receptor, a phenomenon linked to increased risk of cardiometabolic disorders in adulthood. However, the consequences of these early life, cadmium-induced altered DNA methylation patterns on the development of diseases later in life is largely unknown. Therefore, an interesting future course for our model would be to investigate the impact of early-life cadmium exposure and altered DNA methylation patterns on the development of high fat diet-induced NAFLD.

Our data support the literature that cadmium accumulates in the liver in a concentration-dependent manner and this burden is much greater in females compared to males (Vahter et al., 2002; Vahter et al., 2007; Young et al., 2019). Historically, the health effects related to cadmium were thought to be more common among women than men. However, in addition to these ideas being 
driven by observation of greater cadmium body burden in women, they were also formulated based on the epidemiology of Itai-itai disease. Appearing in Japan after World War II due to consumption of cadmium-contaminated rice, Itai-itai disease, which effects the kidneys and bones, was found almost exclusively in elderly multiparous women (Nogawa and Kido, 1996; Ogawa et al., 2004). As such, these studies rarely considered menopause or age-specific sex differences. In fact, in a recent in vivo study Go et al (2015) actually argued that because females absorb cadmium to a greater degree than males, any observation in the males should be either worse or replicable in females and therefore, using only males in their study was acceptable.

By contrast, studies that have taken these factors into consideration show pre-menopausal women have a lower prevalence and incidence of NAFLD compared to men despite having a greater cadmium body burden (Long et al., 2018; Lonardo et al., 2019). Epidemiologically, environmental cadmium exposure is associated with necroinflammation, but not NAFLD in women (Hyder et al., 2013). A study in CD1 female mice showed subchronic exposure to cadmium resulted in upregulated mRNA levels of hepatic lipid metabolism genes but did not result in insulin resistance or lipid accumulation in the liver (Zhang et al., 2017). Similarly, in our study we found whole life exposure to cadmium did not result in insulin resistance or cause hepatic lipid deposition. 
Zinc supplementation rescues cadmium exacerbated, high fat diet-induced NAFLD

Discovering that whole life cadmium exposure interacts with diet to exacerbate NAFLD in male mice, we sought to determine if zinc supplementation could ameliorate the outcome. Zinc is an essential trace element that plays a critical role in various physiological processes, including glucose and lipid metabolism (Olechnowicz et al., 2018; Zhang and Cai, 2020). Thus, several studies have looked at the interactions between obesity and zinc homeostasis. Epidemiologically, serum zinc levels are significantly decreased in obese patients (Peraza et al., 1998; de Luis et al., 2013; Suliburska et al., 2013) and low nutritional zinc status is associated with insulin resistance and an altered lipid profile (Costarelli et al., 2010). These results are supported by animal studies showing zinc deficiency worsens disease outcomes associate with high fat diet and obesity while zinc supplementation alleviates such outcomes (Wang et al., 2016; Luo et al., 2016; Chen et al., 2016; Cooper-Capetini et al., 2017; Qi et al., 2020).

We found zinc supplementation tended to alleviate high fat diet associated insulin resistance, liver injury and hepatic lipid deposition, but not body weight gain. These findings are consistent with the results reported by Qi et al (2020) that found zinc supplementation (90 $\mathrm{mg} / \mathrm{kg}$ zinc) given to mice fed a $60 \% \mathrm{kcal}$ fat diet for 6 weeks did not alter body weight gain over time. In contrast zinc supplementation significantly reduced \% fat mass, of which we did not observe in our study. Furthermore, Qi et al (2020) showed much greater decreases in hepatic triglycerides and the percent areas stained with oil red $\mathrm{O}$ then we observed in our 
model. The variance in the degree of zinc supplemented-rescue between our model and the model reported by Qi and colleagues may be explained by the differences is the duration of the studies (6 weeks vs. 24 weeks) and the age at which the mice were provided both the high fat and zinc supplemented diets ( 3 weeks vs. 8 weeks). However, it is clear from both studies that zinc supplementation can attenuate high fat diet-induced liver disease.

As part of the characterization of our model, we investigated the impact of zinc supplementation on hepatic expression of metallothionein, as this cysteine rich, small molecular weight molecule also plays a critical role in zinc homeostasis (Baltaci et al., 2018). Additionally, zinc supplements are thought to, in part, have their effect by inducing the synthesis of metallothionein (Peraza et al., 1998). Our lab has shown zinc supplementation increased cardiac expression of metallothionein in mice fed normal diet (Wang et al., 2017). However in our current study, zinc supplementation did not change hepatic zinc levels, nor did it induce synthesis of hepatic metallothionein in normal diet fed mice. Interestingly, zinc supplementation has been shown to induce metallothionein in the intestine, which then binds to divalent cations, such as zinc, inhibiting the degree of intestinal uptake and subsequent disruption to other tissues (Brewer et al., 1994; Hoogenraad, 2006). Therefore, in our current model, it is possible that in normal diet fed mice, the additional dietary zinc did not make its way to the liver and therefore did not induce hepatic metallothionein.

In stark contrast, in high fat diet-fed mice, metallothionein mRNA levels were approximately 50-times greater in animals whose diets were supplemented 
with zinc. Furthermore, these results translated to significant increases in metallothionein protein levels which were approximately 17-times greater in zinc supplemented animals fed high fat diet. These results suggest high fat diet creates an environment in which dietary zinc supplementation can increase metallothionein synthesis and are in line with the various studies showing zinc supplementation increases the expression of metallothionein in the tissues of obese animals and subsequent protection from high fat diet-induced diseases (Hennigar et al., 2016; Wang et al., 2017; Olechnowicz et al., 2018).

We found exposure to $5 \mathrm{ppm}$ cadmium increases the severity of high fat diet-induced NAFLD in male mice and were able to rescue this phenotype by supplementing the diet with zinc and significantly decreasing hepatomegaly, plasma ALT levels, steatosis, lipid deposition and hepatic triglycerides. Indeed, the toxic effects of cadmium are highly influenced by zinc status in the body (Brzóska and Moniuszko-Jakoniuk, 2001). Diets deficient in zinc increase cadmium body burden and toxicity (Waalkes et al 1991) while numerous data in laboratory animals indicate the addition of zinc protects against the adverse of effects of cadmium-induced liver injury (Khan et al., 1991; Kudo et al., 1991). We, as well as others, have shown zinc supplementation attenuates high fat diet-induced outcomes (Wang et al., 2016; Luo et al., 2016; Chen et al., 2016; Cooper-Capetini et al., 2017; Qi et al., 2020). However, this is the first study to show zinc supplementation can rescue a cadmium-exacerbated, high fat diet induced NAFLD. 
Our data further suggest zinc supplementation may act through induction of metallothionein to attenuate the consequences of consuming a high fat diet. In high fat diet fed mice, zinc supplementation resulted in increased metallothionein levels (both mRNA and protein) in $5 \mathrm{ppm}$ cadmium exposed mice, although the degree of induction was less than what was seen in the high fat diet fed control mice. Overall, these data implicate the use of zinc supplementation as a possible therapeutic for NAFLD, which may in part act by targeting metallothionein.

\section{Exposure to $0.5 \mathrm{ppm}$ cadmium rescues high fat diet-induced NAFLD in male} mice

Unexpectedly, and in contrast with our observation that whole life exposure to $5 \mathrm{ppm}$ cadmium exacerbated high fat diet-induced NAFLD, exposure to $0.5 \mathrm{ppm}$ cadmium actually rescued NAFLD induced by high fat diet in male mice. In fact, after 24 weeks of being fed high fat diet, mice exposed to $0.5 \mathrm{ppm}$ cadmium had a marked decrease in body weight compared to high fat diet controls that continued until sacrifice. The reduced weight gain was further reflected in improved glucose handling and decreased insulin in the plasma, similar to levels seen in normal diet fed animals. This outcome was not seen in females.

The literature in support of cadmium as a therapeutic is virtually nonexistent. However, there are a few studies that have exploited cadmium's adverse effects on cell proliferation and the immune system in the treatment of rheumatoid arthritis (Ansari et al., 2015; Bonaventura et al., 2017). Interestingly, Ansari et al (2015) showed exposure to 5 ppm cadmium chloride in drinking water restored 
antioxidant levels, arrested progression of the autoimmune disease and downregulated pro-inflammatory modulators whereas exposure to $50 \mathrm{ppm}$ cadmium chloride had the complete opposite effect, increasing the pro-inflammatory response and exacerbating the disease. These results are particularly interesting in that the outcomes at one concentration of cadmium are very different than the outcomes at a 10 times greater concentration - a phenomenon similar to what we observed in our study. Therefore, in our model, the effects of inflammation on NAFLD progression is an important future consideration.

The capacity of cadmium to mimic essential metals may, in part, explain the rescuing effect of $0.5 \mathrm{ppm}$ cadmium in high fat diet-induced NAFLD. Obese individuals have high rates of micronutrient deficiency, despite the excessive intake of food and such deficiencies may contribute to the development of high fat diet-induced disease (Garcia et al., 2009; Via et al., 2012). It is possible that exposure to cadmium at this lower concentration of $0.5 \mathrm{ppm}$ sufficiently acts to seemingly restore the micronutrient deficiency through molecular mimicry and thus reduce the effect of high fat diet. However, further studies would need to be conducted to examine micronutrient status in these animals as well as the long term, later life implications for such an exposure.

The observed protection from high fat diet outcomes may also be explained by metallothionein levels. In normal diet-fed mice, exposure to $0.5 \mathrm{ppm}$ cadmium did not provoke metallothionein synthesis. However, in mice fed high fat diet both mRNA and protein levels of metallothionein increased after exposure to $0.5 \mathrm{ppm}$ cadmium, compared to both controls and 5 ppm cadmium exposed mice. As 
metallothionein plays a major role in essential metal homeostasis, its induction may help elevate high fat diet-associated nutrient deficiencies and thus prevent obesity related diseases. Indeed, animal studies implicate metallothionein in obesity prevention, but the mechanisms remain unclear (Sato et al., 2013; Byun et al., 2011; Lindeque et al., 2015; Kawakami et al., 2019).

It is also worth mentioning that in high fat diet fed mice exposed to $0.5 \mathrm{ppm}$ cadmium, both Srebf-1 mRNA and mature SREBP-1 protein levels were higher compared to controls suggesting the protective effect of $0.5 \mathrm{ppm}$ cadmium on high fat diet-induced NAFLD may be in part due to alterations in SREBP-1 mediated lipogenesis. However, the observed increases in SREBP-1 levels were not reflected in the mRNA of Fasn or Scd-1. Thus, further investigation is needed to determine the involvement of SREBP-1 in the protective mechanism of $0.5 \mathrm{ppm}$ cadmium.

\section{Whole life exposures}

\section{Cadmium and sex}

Although cadmium exposure is associated with the development of several metabolic diseases, such as NAFLD, these studies did not take into account such environmental exposures can be life-long and multigenerational nor did they take into account risk of developing adulthood diseases is correlated with adverse stimuli in utero, resulting in permanent physiological and metabolic changes. Furthermore, although low level, prenatal cadmium exposure is strongly associated with non-lethal birth outcomes, the impact of these early life exposures 
on the development of diseases later in life remain elusive (Young and Cai, 2020). Therefore, our model of whole life exposure to cadmium, starting in utero and continuing through adulthood, provides unique insights into disease initiation and progression.

To date, one other study has investigated the impact of early life exposure to cadmium on hepatic lipid metabolism and fat accumulation in adulthood. Ba et al (2017) exposed both male and female mice to $100 \mathrm{nM}$ (18 ppb) cadmium chloride in drinking water, starting in utero and continuing until 16 weeks postweaning and found a significant increase in body fat, liver triglycerides and lipid deposition in male, but not female mice (Ba et al., 2017). Although the exposure paradigm used by Ba and colleagues (2017) does resemble our study, we obtained very different results. In our study, as mentioned above, cadmium exposure did not alter body composition or measurements of NAFLD in male or female mice.

Importantly, in the study performed by Ba et al. (2017), mice were fed standard laboratory rodent diets with no explanation of the diet composition. These standard rodent diets can vary greatly from batch to batch due to the natural fluctuation of growing seasons and harvest locations of the plant materials used to make the diet (Ricci and Ulman, 2005). Such fluctuations include content of heavy metals, such as cadmium. In fact, cadmium contamination ranging from 30-100 ppb was found in 13 laboratory rodent diets from 5 different continents (Mesnage et al., 2015). Therefore, the mice in the study were likely exposed to much higher levels of cadmium than provided in the drinking water. The mice in our model were fed purified diets in order to avoid such confounding factors. 
High fat diet and sex

In line with the "multiple hit" hypothesis of NAFLD progression, the role of diet-environment interaction in the initiation, progression and development of human metabolic diseases is of growing interest. Animal studies have been published investigating the relationship between diet and low level cadmium exposure, showing that the combination of high fat or high cholesterol diets with cadmium exposure results in increased risk of heart failure and altered bone quality (Türkcan et al., 2015; Zhang et al., 2020). However, in addition to absence of studies investigating diet-cadmium interactions in liver disease, previous studies start their diet regime when rodents are 6 or 8 weeks old, which is comparable to an 11.5 and 20 year old human, respectively. Therefore, these studies do not take into consideration that obesity can start at a much younger age. In fact, in 2016 the incidence of obesity in children between the ages of 2-5 in the United States was $13.9 \%$ and at least $25 \%$ of these children are at risk for remaining obese into adulthood (Sanyaolu et al., 2019). In our model, mice were started on high fat diet when they were weaned, at 3 weeks of age ( 6 month old human), thus taking into consideration exposure to diets high in fat can occur early in life and continue through adulthood.

In male mice, consumption of a high fat diet for 24 weeks, starting at 3 weeks of age resulted in increased body weight, percent fat, lean tissue mass, and blood glucose and plasma insulin levels. Furthermore, this high fat diet feeding regime resulted in hepatomegaly, increased levels of plasma ALT, steatosis and 
increased hepatic triglycerides. Our results are consistent with other high fat dietinduced models of NAFLD in male (Nakamura and Terauchi, 2013; Recena Aydos et al., 2019; Velázquez et al., 2019)

In our model, consumption of high fat diet did not trigger a SREBP-1 mediated increase in lipid synthesis in male mice. In fact, in our model, high fat diet decreased two rate limiting lipogenic enzymes, suggesting an overall decrease in SREBP-1-mediated in lipid synthesis. Our results contradict the existing literature (Biddinger et al., 2005; Buettner et al., 2006) that show high fat-diet increases SREBP-1-mediated lipid synthesis. For example, in male mice fed a high fat diet (55\% calories from fat) for 18 weeks starting at 6 weeks of age, activation of SREBP-1 and SCD-1 transcript levels and activity were increased (Biddinger et al., 2005). Similarly, in male rats fed a western diet ( $42 \%$ calories from fat) for 12 weeks, also starting at 6 weeks of age, Buettner and colleagues (2006) found an upregulation of hepatic SREBP-1c-dependent genes.

Our outcomes in male animals exposed to high fat diet alone are consistent with these previous reports, thus the reason for the discrepancies in levels of SREBP-1 and its downstream lipogenic targets between our results and the existing literature is uncertain. One explanation may be the length of the study and the age at which the animals were given access to high fat diet. In the previous studies, animals were started on a high fat diet at 6 weeks of age, in contrast, in our model, the pups were moved onto a high fat diet at the time weaning (approximately 3 weeks of age). Although a difference of 3-5 weeks may not seem like a long time, in "mouse days" it can be the difference between puberty and 
adulthood. A 3, 6, and 8-10 week old mouse is comparable to a 6 month old, 11.5 year old, and 20 year old human, respectively (Dutta and Sengupta, 2016). Therefore, age-induced metabolic changes may account for these discrepancies. However the data supporting age-related discrepancies under the age of 20 are few as most of them examine the differences between adults of reproductive age and elderly populations (Roberts et al., 2006). One study, in humans, did examine incidence of obesity-induced metabolic syndrome and found increases with age from $3 \%$ to $9 \%$ in adolescents ages 13 and 19 , respectively (Sinaiko et al., 2005).

In females, we showed 24 weeks of high fat diet feeding increased bodyweight gain, percent fat and induced hyperglycemia without changes in plasma insulin levels in female mice. These results were similar to those found in a study by Pettersson et al (2012) who showed 14 weeks of high fat diet feeding in female mice caused weight gain and impaired glucose tolerance, while normal serum insulin concentrations were maintained. We further showed that although measures of hepatic injury were not altered as a result of high fat diet consumption, lipid deposition and hepatic triglycerides were increased, indicating our high fat diet model induced steatosis. These results are consistent with a study by Ganz et al (2014) who found 16 weeks of high fat diet feeding resulted in increased hepatic triglycerides and steatosis, but no change in plasma ALT levels in female mice. Taken together, our study extends the field to show whole life exposure to cadmium does not induce NAFLD in female animals, which recapitulates the disease etiology seen in human populations. 


\section{Limitations}

While the results from this study provide critical insight into the "multiple-hit" nature of NAFLD, considering diet, environmental exposures and sex as contributing variables there are several limitations in this study. First, due to budgetary restraints, DEXAscans were not performed on mice exposed to $0.5 \mathrm{ppm}$ cadmium as we originally expected that the greatest effect would be with $5 \mathrm{ppm}$ cadmium, and to a lesser degree $0.5 \mathrm{ppm}$ exposure. We however did not anticipate the stark, opposite outcomes between the two cadmium exposures. Therefore, additional information of effects on body mass composition in $0.5 \mathrm{ppm}$ cadmium exposed mice would be informative. Furthermore, this study only investigates the impact of two concentrations of cadmium in disease outcome, thus not providing an informative dose response curve. Additional cadmium exposures would possibly provide valuable insight into the opposing cadmium effects we observed in our model. Another limitation is the number of animals in the study. In some of the exposure groups there was a small number of animals, therefore statistical power was low in these groups. A greater number of animals per a group would possibly clarify some of the observed variability in the model. 


\section{Future Directions}

While studies have looked at the impact of diet and cadmium exposure as independent variables, our study is among the first to investigate how these two factors may act together to influence initiation and progression of NAFLD. Our data show that while in male mice exposure to $5 \mathrm{ppm}$ cadmium exacerbated high fat diet-induced NAFLD and exposure to $0.5 \mathrm{ppm}$ cadmium reversed the phenotype, in female mice cadmium exposure did not alter the observed high fat diet associated outcomes. Further investigation is needed to understand the underlying mechanism responsible for the sexual dimorphism in our model, leading the way to more target therapies. Indeed, there is evidence that cadmium has estrogenic effects and can act as an endocrine disrupter (Vahter et al., 2007; Nasiadek et al., 2018) so it would be interesting to perform our study in ovariectomized mice.

Furthermore, our data show zinc levels in high fat diet fed female mice are increased in response to cadmium exposure, a possible mechanism by which females were protected from the cadmium-exacerbated, high fat diet effects observed in male mice. Therefore, it would be of interest to introduce a zinc deficient diet into our exposure model to determine 1) does zinc deficiency in female mice increase their susceptibility to cadmium-altered NAFLD and 2) does zinc deficiency in male mice result in further exacerbation or altered NAFLD pathogenesis?

Our data show the mechanism by which 5 ppm cadmium exacerbates high fat diet-induced NAFLD was not through altered de novo lipogenesis; however, we did not look at the other side of the lipid metabolism pathway, fatty acid $\beta$-oxidation. 
Although there are limited data implicating cadmium's ability to alter the breakdown of fatty acids, it would be important to investigate the contribution of this pathway to the pathology observed in this study.

To our surprise, our data show exposure to $0.5 \mathrm{ppm}$ cadmium recues high fat-induced NAFLD. The mechanism of cadmium-associated disease recovery undoubtedly deserved further investigation. As such, future studies may include investigating the role of cadmium and inflammation in NAFLD pathogenesis. This future direction is based on a report showing exposure to $5 \mathrm{ppm}$ cadmium stopped the progression of rheumatoid arthritis by restoring antioxidant levels and downregulating the pro-inflammatory response (Ansari et al., 2015).

Lastly, in addition to the liver, our model included the collection of other metabolic organs, providing for the opportunity to investigate organ crosstalk in disease development. Of particular interest to our lab is the cross-talk between the liver and the heart. Interestingly, cardiovascular diseases are the most common cause of mortality in patients with NAFLD (Targher et al., 2010). Furthermore, we have recently shown the combination of chronic cadmium exposure and high fat diet result in cardiac remodeling (Liang et al., 2019). However, the impact of cadmium-altered, high fat diet-induced NAFLD and the subsequent increased risk of CVD has not been investigated. 


\section{REFERENCES}

Alegría-Torres, J. A., Carrizales-Yánez, L., Díaz-Barriga, F., Rosso-Camacho, F., Motta, V., Tarantini, L., \& Bollati, V. (2016). DNA methylation changes in Mexican children exposed to arsenic from two historic mining areas in San Luis potosí. Environmental and molecular mutagenesis, 57(9), 717-723. https://doi.org/10.1002/em.22062

Akinloye, O., Ogunleye, K., Oguntibeju, O.O. (2010). Cadmium, lead, arsenic and selenium levels in patients with type 2 diabetes mellitus. African journal of biotechnology, 9 (32), 5189-5195.

Aoun, M., Fouret, G., Michel, F., Bonafos, B., Ramos, J., Cristol, J. P., Carbonneau, M. A., Coudray, C., \& Feillet-Coudray, C. (2012). Dietary fatty acids modulate liver mitochondrial cardiolipin content and its fatty acid composition in rats with non alcoholic fatty liver disease. Journal of bioenergetics and biomembranes, 44(4), 439-452. https://doi.org/10.1007/s10863-012-9448-x

Ansari, M. M., Neha, \& Khan, H. A. (2015). Effect of cadmium chloride exposure during the induction of collagen induced arthritis. Chemico-biological interactions, 238, 55-65. https://doi.org/10.1016/j.cbi.2015.06.001

Arnold A. P. (2010). Promoting the understanding of sex differences to enhance equity and excellence in biomedical science. Biology of sex differences, 1(1), 1. https://doi.org/10.1186/2042-6410-1-1

Arroyo, V.S., Flores, K.M., Ortiz, L.B., Gómez-Quiroz, L.E., Gutiérrez-Ruiz, M.C. (2012). Liver and Cadmium Toxicity. Journal of Drug Metabolism \& Toxicology. S5:001. doi:10.4172/2157-7609.S5-001

Asrih, M., \& Jornayvaz, F. R. (2015). Metabolic syndrome and nonalcoholic fatty liver disease: Is insulin resistance the link? Molecular and cellular endocrinology, 418 Pt 1, 55-65. https://doi.org/10.1016/..mce.2015.02.018

ATSDR, 2011. Agency for Toxic Substances and Disease Registry Case Studies in Environmental Medicine (CSEM). Cadmium Toxicity. Course WB: 1096. https://www.atsdr.cdc.gov/csem/cadmium/docs/cadmium.pdf 
ATSDR, 2017. Substance Priority List. U.S. Department of Health and Human Services. Public Health Agency for Toxic Substances and Disease Registry.

Baltaci, A. K., Yuce, K., \& Mogulkoc, R. (2018). Zinc Metabolism and Metallothioneins. Biological trace element research, 183(1), 22-31. https://doi.org/10.1007/s12011-017-1119-7

Barker, D. J., \& Osmond, C. (1986). Diet and coronary heart disease in England and Wales during and after the second world war. Journal of epidemiology and community health, 40(1), 37-44. https://doi.org/10.1136/jech.40.1.37

Barker, D. J., Winter, P. D., Osmond, C., Margetts, B., \& Simmonds, S. J. (1989). Weight in infancy and death from ischaemic heart disease. Lancet (London, England), 2(8663), 577-580. https://doi.org/10.1016/s0140$\underline{6736(89) 90710-1}$

Barker, D. J., Gluckman, P. D., Godfrey, K. M., Harding, J. E., Owens, J. A., \& Robinson, J. S. (1993). Fetal nutrition and cardiovascular disease in adult life. Lancet (London, England), 341(8850), 938-941. https://doi.org/10.1016/0140-6736(93)912

Barker, D. J., Eriksson, J. G., Forsén, T., \& Osmond, C. (2002). Fetal origins of adult disease: strength of effects and biological basis. International journal of epidemiology, 31(6), 1235-1239. https://doi.org/10.1093/ije/31.6.1235

Barouki, R., Gluckman, P. D., Grandjean, P., Hanson, M., \& Heindel, J. J. (2012). Developmental origins of non-communicable disease: implications for research and public health. Environmental health : a global access science source, 11, 42. https://doi.org/10.1186/1476-069X-11-42

Bayol, S. A., Simbi, B. H., Fowkes, R. C., \& Stickland, N. C. (2010). A maternal "junk food" diet in pregnancy and lactation promotes nonalcoholic Fatty liver disease in rat offspring. Endocrinology, 151(4), 1451-1461. https://doi.org/10.1210/en.2009-1192

Biddinger, S. B., Almind, K., Miyazaki, M., Kokkotou, E., Ntambi, J. M., \& Kahn, C. R. (2005). Effects of diet and genetic background on sterol regulatory element-binding protein-1c, stearoyl-CoA desaturase 1 , and the development of the metabolic syndrome. Diabetes, 54(5), 1314-1323. https://doi.org/10.2337/diabetes.54.5.1314

Bonaventura, P., Courbon, G., Lamboux, A., Lavocat, F., Marotte, H., Albarède, F., \& Miossec, P. (2017). Protective effect of low dose intra-articular cadmium on inflammation and joint destruction in arthritis. Scientific reports, 7(1), 2415. https://doi.org/10.1038/s41598-017-02611-5 
Bonaventura, P., Lamboux, A., Albarède, F., \& Miossec, P. (2017). Regulatory effects of zinc on cadmium-induced cytotoxicity in chronic inflammation. PloS one, 12(7), e0180879. https://doi.org/10.1371/journal.pone.0180879

Bowe, J. E., Franklin, Z. J., Hauge-Evans, A. C., King, A. J., Persaud, S. J., \& Jones, P. M. (2014). Metabolic phenotyping guidelines: assessing glucose homeostasis in rodent models. The Journal of endocrinology, 222(3), G13G25. https://doi.org/10.1530/JOE-14-0182

Brewer, G. J., Dick, R. D., Yuzbasiyan-Gurkan, V., Johnson, V., \& Wang, Y. (1994). Treatment of Wilson's disease with zinc. XIII: Therapy with zinc in presymptomatic patients from the time of diagnosis. The Journal of laboratory and clinical medicine, 123(6), 849-858.

Brzóska, M. M., \& Moniuszko-Jakoniuk, J. (2001). Interactions between cadmium and zinc in the organism. Food and chemical toxicology : an international journal published for the British Industrial Biological Research Association, 39(10), 967-980. https://doi.org/10.1016/s0278-6915(01)00048-5

Buettner, R., Parhofer, K. G., Woenckhaus, M., Wrede, C. E., Kunz-Schughart, L. A., Schölmerich, J., \& Bollheimer, L. C. (2006). Defining high-fat-diet rat models: metabolic and molecular effects of different fat types. Journal of molecular endocrinology, 36(3), 485-501. https://doi.org/10.1677/jme.1.01909

Buzzetti, E., Pinzani, M., \& Tsochatzis, E. A. (2016). The multiple-hit pathogenesis of non-alcoholic fatty liver disease (NAFLD). Metabolism: clinical and experimental, $65(8)$, 1038-1048. https://doi.org/10.1016/..metabol.2015.12.012

Byun, H. R., Kim, D. K., \& Koh, J. Y. (2011). Obesity and downregulated hypothalamic leptin receptors in male metallothionein-3-null mice. Neurobiology of disease, 44(1), 125-132. https://doi.org/10.1016/j.nbd.2011.06.012

Byrne, C. D., \& Targher, G. (2015). NAFLD: a multisystem disease. Journal of hepatology, 62(1 Suppl), S47-S64. https://doi.org/10.1016/j.jhep.2014.12.

Cardoso, A. R., Kakimoto, P. A., \& Kowaltowski, A. J. (2013). Diet-sensitive sources of reactive oxygen species in liver mitochondria: role of very long chain acyl-CoA dehydrogenases. PloS one, 8(10), e77088. https://doi.org/10.1371/journal.pone.0077088

Castillo, P., Ibáñez, F., Guajardo, A., Llanos, M. N., \& Ronco, A. M. (2012). Impact of cadmium exposure during pregnancy on hepatic glucocorticoid receptor 
methylation and expression in rat fetus. PloS one, 7(9), e44139. https://doi.org/10.1371/journal.pone.0044139

Chalasani, N., Younossi, Z., Lavine, J. E., Diehl, A. M., Brunt, E. M., Cusi, K., Charlton, M., Sanyal, A. J., American Gastroenterological Association, American Association for the Study of Liver Diseases, \& American College of Gastroenterology (2012). The diagnosis and management of nonalcoholic fatty liver disease: practice guideline by the American Gastroenterological Association, American Association for the Study of Liver Diseases, and American College of Gastroenterology. Gastroenterology, 142(7), 1592-1609. https://doi.org/10.1053/i.gastro.2012.04.001

Chen, J., Wang, S., Luo, M., Zhang, Z., Dai, X., Kong, M., Cai, L., Wang, Y., Shi, B., \& Tan, Y. (2016). From the Cover: Zinc Deficiency Worsens and Supplementation Prevents High-Fat Diet Induced Vascular Inflammation, Oxidative Stress, and Pathological Remodeling. Toxicological sciences: an official journal of the Society of Toxicology, 153(1), 124-136. https://doi.org/10.1093/toxsci/kfw110

Chen, Z., Myers, R., Wei, T., Bind, E., Kassim, P., Wang, G., Ji, Y., Hong, X., Caruso, D., Bartell, T., Gong, Y., Strickland, P., Navas-Acien, A., Guallar, E., \& Wang, X. (2014). Placental transfer and concentrations of cadmium, mercury, lead, and selenium in mothers, newborns, and young children. Journal of exposure science \& environmental epidemiology, 24(5), 537544. https://doi.org/10.1038/jes.2014.26

Choudhury, H., Harvey, T., Thayer, W. C., Lockwood, T. F., Stiteler, W. M., Goodrum, P. E., Hassett, J. M., \& Diamond, G. L. (2001). Urinary cadmium elimination as a biomarker of exposure for evaluating a cadmium dietary exposure--biokinetics model. Journal of toxicology and environmental health. Part A, 63(5), 321-350. https://doi.org/10.1080/15287390152103643

Clayton, J. A., \& Collins, F. S. (2014). Policy: NIH to balance sex in cell and animal studies. Nature, 509(7500), 282-283. https://doi.org/10.1038/509282a

Costarelli, L., Muti, E., Malavolta, M., Cipriano, C., Giacconi, R., Tesei, S., Piacenza, F., Pierpaoli, S., Gasparini, N., Faloia, E., Tirabassi, G., Boscaro, M., Polito, A., Mauro, B., Maiani, F., Raguzzini, A., Marcellini, F., Giuli, C., Papa, R., Emanuelli, M., ... Mocchegiani, E. (2010). Distinctive modulation of inflammatory and metabolic parameters in relation to zinc nutritional status in adult overweight/obese subjects. The Journal of nutritional biochemistry, 21(5), 432-437. https://doi.org/10.1016/j.jnutbio.2009.02.001 
Cotzias, G.C., \& Papavasiliou, P.S. (1964). Specificity of zinc pathway through the body: homeostatic considerations. The American journal of physiology, 206, 787-792. https://doi.org/10.1152/ajplegacy.1964.206.4.787

Dakin, R. S., Walker, B. R., Seckl, J. R., Hadoke, P. W., \& Drake, A. J. (2015). Estrogens protect male mice from obesity complications and influence glucocorticoid metabolism. International journal of obesity (2005), 39(10), 1539-1547. https://doi.org/10.1038/ijo.2015.102

Day, C. P., \& James, O. F. (1998). Steatohepatitis: a tale of two "hits"?. Gastroenterology, 114(4), 842-845. https://doi.org/10.1016/s0016$\underline{\text { 5085(98)70599-2 }}$

de Luis, D. A., Pacheco, D., Izaola, O., Terroba, M. C., Cuellar, L., \& Cabezas, G. (2013). Micronutrient status in morbidly obese women before bariatric surgery. Surgery for obesity and related diseases: official journal of the American Society for Bariatric Surgery, 9(2), 323-327. https://doi.org/10.1016/j.soard.2011.09.015

de Rooij, S. R., Painter, R. C., Phillips, D. I., Osmond, C., Michels, R. P., Godsland, I. F., Bossuyt, P. M., Bleker, O. P., \& Roseboom, T. J. (2006). Impaired insulin secretion after prenatal exposure to the Dutch famine. Diabetes care, 29(8), 1897-1901. https://doi.org/10.2337/dc06-0460

Duarte, J. A., Carvalho, F., Pearson, M., Horton, J. D., Browning, J. D., Jones, J. G., \& Burgess, S. C. (2014). A high-fat diet suppresses de novo lipogenesis and desaturation but not elongation and triglyceride synthesis in mice. Journal of lipid research, 55(12), 2541-2553. https://doi.org/10.1194/jlr.M052308

Dutta, S., \& Sengupta, P. (2016). Men and mice: Relating their ages. Life sciences, 152, 244-248. https://doi.org/10.1016/j.lfs.2015.10.025

Fakhouri, T. H., Ogden, C. L., Carroll, M. D., Kit, B. K., \& Flegal, K. M. (2012). Prevalence of obesity among older adults in the United States, 2007-2010. NCHS data brief, (106), 1-8.

Fatima, G., Raza, A. M., Hadi, N., Nigam, N., \& Mahdi, A. A. (2019). Cadmium in Human Diseases: It's More than Just a Mere Metal. Indian journal of clinical biochemistry : IJCB, 34(4), 371-378. https://doi.org/10.1007/s12291-019$\underline{00839-8}$

Ficková, M., Eybl, V., Kotyzová, D., Micková, V., Möstbök, S., \& Brtko, J. (2003). Long lasting cadmium intake is associated with reduction of insulin receptors in rat adipocytes. Biometals : an international journal on the role 
of metal ions in biology, biochemistry, and medicine, 16(4), 561-566. https://doi.org/10.1023/a:1023485130767

Ferré, P., \& Foufelle, F. (2007). SREBP-1c transcription factor and lipid homeostasis: clinical perspective. Hormone research, 68(2), 72-82. https://doi.org/10.1159/000100426

Foldspang, A., \& Hansen, J. C. (1990). Dietary intake of methylmercury as a correlate of gestational length and birth weight among newborns in Greenland. American journal of epidemiology, 132(2), 310-317. https://doi.org/10.1093/oxfordjournals.aje.a115660

Fon Tacer, K., \& Rozman, D. (2011). Nonalcoholic Fatty liver disease: focus on lipoprotein and lipid deregulation. Journal of lipids, 2011, 783976. https://doi.org/10.1155/2011/783976

Foster, M., Hancock, D., Petocz, P., \& Samman, S. (2011). Zinc transporter genes are coordinately expressed in men and women independently of dietary or plasma zinc. The Journal of nutrition, 141(6), 1195-1201. https://doi.org/10.3945/in.111.140053

Fujishiro, H., Okugaki, S., Kubota, K., Fujiyama, T., Miyataka, H., \& Himeno, S. (2009). The role of ZIP8 down-regulation in cadmium-resistant metallothionein-null cells. Journal of applied toxicology: JAT, 29(5), 367373. https://doi.org/10.1002/jat.1419

Fukunaka, A., \& Fujitani, Y. (2018). Role of Zinc Homeostasis in the Pathogenesis of Diabetes and Obesity. International journal of molecular sciences, 19(2), 476. https://doi.org/10.3390/ijms19020476

Funk, A. E., Day, F. A., \& Brady, F. O. (1987). Displacement of zinc and copper from copper-induced metallothionein by cadmium and by mercury: in vivo and ex vivo studies. Comparative biochemistry and physiology. C, Comparative pharmacology and toxicology, 86(1), 1-6. https://doi.org/10.1016/0742-8413(87)90133-2

Ganz, M., Csak, T., \& Szabo, G. (2014). High fat diet feeding results in gender specific steatohepatitis and inflammasome activation. World journal of gastroenterology, 20(26), 8525-8534. https://doi.org/10.3748/wjg.v20.i26.8525

García, O. P., Long, K. Z., \& Rosado, J. L. (2009). Impact of micronutrient deficiencies on obesity. Nutrition reviews, 67(10), 559-572. https://doi.org/10.1111/j.1753-4887.2009.00228.x 
George, V., Tremblay, A., Després, J. P., Leblanc, C., \& Bouchard, C. (1990). Effect of dietary fat content on total and regional adiposity in men and women. International journal of obesity, 14(12), 1085-1094.

Ghasemi, A., Zahediasl, S., Hosseini-Esfahani, F., \& Azizi, F. (2014). Gender differences in the relationship between serum zinc concentration and metabolic syndrome. Annals of human biology, 41(5), 436-442. https://doi.org/10.3109/03014460.2013.870228

Gluckman, P. D., Hanson, M. A., \& Mitchell, M. D. (2010). Developmental origins of health and disease: reducing the burden of chronic disease in the next generation. Genome medicine, 2(2), 14. https://doi.org/10.1186/gm135

Go, Y. M., Sutliff, R. L., Chandler, J. D., Khalidur, R., Kang, B. Y., Anania, F. A., Orr, M., Hao, L., Fowler, B. A., \& Jones, D. P. (2015). Low-Dose Cadmium Causes Metabolic and Genetic Dysregulation Associated With Fatty Liver Disease in Mice. Toxicological sciences: an official journal of the Society of Toxicology, 147(2), 524-534. https://doi.org/10.1093/toxsci/kfv149

Golay, A., \& Bobbioni, E. (1997). The role of dietary fat in obesity. International journal of obesity and related metabolic disorders: journal of the International Association for the Study of Obesity, 21 Suppl 3, S2-S11.

Gonzalez-Reimers, E., Martín-González, C., Galindo-Martín, L., Aleman-Valls, M. R., Velasco-Vázquez, J., Arnay-de-la-Rosa, M., Pérez-Hernández, O., \& Luis, R. H. (2014). Lead, cadmium and zinc in hair samples: relationship with dietary habits and urban environment. Biological trace element research, 157(3), 205-210. https://doi.org/10.1007/s12011-014-9896-8

Gu, J., Cheng, Y., Wu, H., Kong, L., Wang, S., Xu, Z., Zhang, Z., Tan, Y., Keller, B. B., Zhou, H., Wang, Y., Xu, Z., \& Cai, L. (2017). Metallothionein Is Downstream of Nrf2 and Partially Mediates Sulforaphane Prevention of Diabetic Cardiomyopathy. Diabetes, 66(2), 529-542. https://doi.org/10.2337/db15-1274

Habeebu, S. S., Liu, J., Liu, Y., \& Klaassen, C. D. (2000). Metallothionein-null mice are more susceptible than wild-type mice to chronic $\mathrm{CdCl}(2)$-induced bone injury. Toxicological sciences, 56(1), 211-219. https://doi.org/10.1093/toxsci/56.1.211

Hales, C. M., Carroll, M. D., Fryar, C. D., \& Ogden, C. L. (2020). Prevalence of Obesity and Severe Obesity Among Adults: United States, 2017-2018. NCHS data brief, (360), 1-8.

Handgraaf, S., Riant, E., Fabre, A., Waget, A., Burcelin, R., Lière, P., Krust, A., Chambon, P., Arnal, J. F., \& Gourdy, P. (2013). Prevention of obesity and 
insulin resistance by estrogens requires ERa activation function-2 (ERaAF2), whereas ERaAF-1 is dispensable. Diabetes, 62(12), 4098-4108. https://doi.org/10.2337/db13-0282

Hariri, N., \& Thibault, L. (2010). High-fat diet-induced obesity in animal models. Nutrition research reviews, 23(2), 270-299. https://doi.org/10.1017/S0954422410000168

Hazardous substances database (HSDB). 2006. Cadmium. National Library of Medicine Toxicology Data network, http://toxnet.nlm.nih.gov

He, W., Guo, W., Qian, Y., Zhang, S., Ren, D., \& Liu, S. (2015). Synergistic hepatotoxicity by cadmium and chlorpyrifos: disordered hepatic lipid homeostasis. Molecular medicine reports, 12(1), 303-308. https://doi.org/10.3892/mmr.2015.3381

He, X., Gao, J., Hou, H., Qi, Z., Chen, H., \& Zhang, X. X. (2019). Inhibition of Mitochondrial Fatty Acid Oxidation Contributes to Development of Nonalcoholic Fatty Liver Disease Induced by Environmental Cadmium Exposure. Environmental science \& technology, 53(23), 13992-14000. https://doi.org/10.1021/acs.est.9b05131

Heindel, J. J., Balbus, J., Birnbaum, L., Brune-Drisse, M. N., Grandjean, P., Gray, K., Landrigan, P. J., Sly, P. D., Suk, W., Cory Slechta, D., Thompson, C., \& Hanson, M. (2015). Developmental Origins of Health and Disease: Integrating Environmental Influences. Endocrinology, 156(10), 3416-3421. https://doi.org/10.1210/EN.2015-1394

Heindel, J. J., Skalla, L. A., Joubert, B. R., Dilworth, C. H., \& Gray, K. A. (2017). Review of developmental origins of health and disease publications in environmental epidemiology. Reproductive toxicology (Elmsford, N.Y.), 68, 34-48. https://doi.org/10.1016/..reprotox.2016.11.011

Hennigar, S. R., Kelley, A. M., \& McClung, J. P. (2016). Metallothionein and Zinc Transporter Expression in Circulating Human Blood Cells as Biomarkers of Zinc Status: a Systematic Review. Advances in nutrition (Bethesda, Md.), 7(4), 735-746. https://doi.org/10.3945/an.116.012518

Heymsfield, S. B., \& Cefalu, W. T. (2013). Does body mass index adequately convey a patient's mortality risk?. JAMA, 309(1), 87-88. https://doi.org/10.1001/jama.2012.185445

Hoogenraad T. U. (2006). Paradigm shift in treatment of Wilson's disease: zinc therapy now treatment of choice. Brain \& development, 28(3), 141-146. https://doi.org/10.1016/j.braindev.2005.08.008 
Hudson, K. M., Belcher, S. M., \& Cowley, M. (2019). Maternal cadmium exposure in the mouse leads to increased heart weight at birth and programs susceptibility to hypertension in adulthood. Scientific reports, 9(1), 13553. https://doi.org/10.1038/s41598-019-49807-5

Huen, K., Yousefi, P., Bradman, A., Yan, L., Harley, K. G., Kogut, K., Eskenazi, B., \& Holland, N. (2014). Effects of age, sex, and persistent organic pollutants on DNA methylation in children. Environmental and molecular mutagenesis, 55(3), 209-222. https://doi.org/10.1002/em.21845

Hyder, O., Chung, M., Cosgrove, D., Herman, J. M., Li, Z., Firoozmand, A., Gurakar, A., Koteish, A., \& Pawlik, T. M. (2013). Cadmium exposure and liver disease among US adults. Journal of gastrointestinal surgery: official journal of the Society for Surgery of the Alimentary Tract, 17(7), 1265-1273. https://doi.org/10.1007/s11605-013-2210-9

IARC. 1993. Cadmium and cadmium compounds. In Beryllium, Cadmium, Mercury and Exposures in the Glass Manufacturing Industry. IARC Monographs on the Evaluation of Carcinogenic Risk of Chemicals to Humans, vol. 58. Lyon, France: International Agency for Research on Cancer. pp. 119-239.

Integrated risk information system (IRIS). (2006). US EPA, Office of Research and Development, National Center for Environmental Assessment.

IPCS, 1992. Cadmium - Environmental aspects. Geneva, World Health Organization, International Programme on Chemical Safety. Environmental Health Criteria 135 ; http://www.inchem.org/documents/ehc/ehc/ehc135.htm (accessed 30 April 2020)

Ipsen, D. H., Lykkesfeldt, J., \& Tveden-Nyborg, P. (2018). Molecular mechanisms of hepatic lipid accumulation in non-alcoholic fatty liver disease. Cellular and molecular life sciences: CMLS, 75(18), 3313-3327. https://doi.org/10.1007/s00018-018-2860-6

Jacobo-Estrada, T., Santoyo-Sánchez, M., Thévenod, F., \& Barbier, O. (2017). Cadmium Handling, Toxicity and Molecular Targets Involved during Pregnancy: Lessons from Experimental Models. International journal of molecular sciences, 18(7), 1590. https://doi.org/10.3390/ijms18071590

Järup, L., \& Akesson, A. (2009). Current status of cadmium as an environmental health problem. Toxicology and applied pharmacology, 238(3), 201-208. https://doi.org/10.1016/j.taap.2009.04.020 
Järup, L., Berglund, M., Elinder, C. G., Nordberg, G., \& Vahter, M. (1998). Health effects of cadmium exposure--a review of the literature and a risk estimate. Scandinavian journal of work, environment \& health, 24 Suppl 1, 1-51.

Jazwiec, P. A., \& Sloboda, D. M. (2019). Nutritional adversity, sex and reproduction: 30 years of $\mathrm{DOHaD}$ and what have we learned?. The Journal of endocrinology, 242(1), T51-T68. https://doi.org/10.1530/JOE-19-0048

Kang, M. Y., Cho, S. H., Lim, Y. H., Seo, J. C., \& Hong, Y. C. (2013). Effects of environmental cadmium exposure on liver function in adults. Occupational and environmental medicine, 70(4), 268-273. https://doi.org/10.1136/oemed-2012-101063

Kawakami, T., Takasaki, S., Kadota, Y., Fukuoka, D., Sato, M., \& Suzuki, S. (2019). Regulatory role of metallothionein-1/2 on development of sex differences in a high-fat diet-induced obesity. Life sciences, 226, 12-21. https://doi.org/10.1016/j.Ifs.2019.04.012

Kawakami, T., Nishiyama, K., Kadota, Y., Sato, M., Inoue, M., \& Suzuki, S. (2013). Cadmium modulates adipocyte functions in metallothionein-null mice. Toxicology and applied pharmacology, 272(3), 625-636. https://doi.org/10.1016/j.taap.2013.07.015

Kelishadi, R., Askarieh, A., Motlagh, M. E., Tajadini, M., Heshmat, R., Ardalan, G., Fallahi, S., \& Poursafa, P. (2013). Association of blood cadmium level with cardiometabolic risk factors and liver enzymes in a nationally representative sample of adolescents: the CASPIAN-III study. Journal of environmental and public health, 2013, 142856. https://doi.org/10.1155/2013/142856

Kennedy, A. P., Shea, J. L., \& Sun, G. (2009). Comparison of the classification of obesity by BMI vs. dual-energy X-ray absorptiometry in the Newfoundland population. Obesity (Silver Spring, Md.), 17(11), 2094-2099. https://doi.org/10.1038/oby.2009.101

Kim, K., Melough, M. M., Vance, T. M., Noh, H., Koo, S. I., \& Chun, O. K. (2018). Dietary Cadmium Intake and Sources in the US. Nutrients, 11(1), 2. https://doi.org/10.3390/nu11010002

Kippler, M., Engström, K., Mlakar, S. J., Bottai, M., Ahmed, S., Hossain, M. B., Raqib, R., Vahter, M., \& Broberg, K. (2013). Sex-specific effects of early life cadmium exposure on DNA methylation and implications for birth weight. Epigenetics, 8(5), 494-503. https://doi.org/10.4161/epi.24401

Kirsch, R., Clarkson, V., Shephard, E. G., Marais, D. A., Jaffer, M. A., Woodburne, V. E., Kirsch, R. E., \& Hall, P. (2003). Rodent nutritional model of nonalcoholic steatohepatitis: species, strain and sex difference studies. Journal 
of gastroenterology and hepatology, 18(11), 1272-1282. https://doi.org/10.1046/j.1440-1746.2003.03198.x

Klaassen, C. D., Liu, J., \& Choudhuri, S. (1999). Metallothionein: an intracellular protein to protect against cadmium toxicity. Annual review of pharmacology and toxicology, 39, 267-294. https://doi.org/10.1146/annurev.pharmtox.39.1.267

Klaassen, C. D., Liu, J., \& Diwan, B. A. (2009). Metallothionein protection of cadmium toxicity. Toxicology and applied pharmacology, 238(3), 215-220. https://doi.org/10.1016/j.taap.2009.03.026

Kleiner, D. E., Brunt, E. M., Van Natta, M., Behling, C., Contos, M. J., Cummings, O. W., Ferrell, L. D., Liu, Y. C., Torbenson, M. S., Unalp-Arida, A., Yeh, M., McCullough, A. J., Sanyal, A. J., \& Nonalcoholic Steatohepatitis Clinical Research Network (2005). Design and validation of a histological scoring system for nonalcoholic fatty liver disease. Hepatology (Baltimore, Md.), 41(6), 1313-1321. https://doi.org/10.1002/hep.20701

Kohjima, M., Enjoji, M., Higuchi, N., Kato, M., Kotoh, K., Yoshimoto, T., Fujino, T., Yada, M., Yada, R., Harada, N., Takayanagi, R., \& Nakamuta, M. (2007). Re-evaluation of fatty acid metabolism-related gene expression in nonalcoholic fatty liver disease. International journal of molecular medicine, 20(3), 351-358.

Koo S. H. (2013). Nonalcoholic fatty liver disease: molecular mechanisms for the hepatic steatosis. Clinical and molecular hepatology, 19(3), 210-215. https://doi.org/10.3350/cmh.2013.19.3.210

Kotronen, A., Seppänen-Laakso, T., Westerbacka, J., Kiviluoto, T., Arola, J., Ruskeepää, A. L., Oresic, M., \& Yki-Järvinen, H. (2009). Hepatic stearoylCoA desaturase (SCD)-1 activity and diacylglycerol but not ceramide concentrations are increased in the nonalcoholic human fatty liver. Diabetes, 58(1), 203-208. https://doi.org/10.2337/db08-1074

Kurowska, E., \& Bal, W. (2010). Chapter 3 - Recent Advances in Molecular Toxicology of Cadmium and Nickel. Advances in Molecular Toxicology, 4, 85-126.

Lazo, M., Hernaez, R., Eberhardt, M. S., Bonekamp, S., Kamel, I., Guallar, E., Koteish, A., Brancati, F. L., \& Clark, J. M. (2013). Prevalence of nonalcoholic fatty liver disease in the United States: the Third National Health and Nutrition Examination Survey, 1988-1994. American journal of epidemiology, 178(1), 38-45. https://doi.org/10.1093/aje/kws448 
Levy, J. R., Gyarmati, J., Lesko, J. M., Adler, R. A., \& Stevens, W. (2000). Dual regulation of leptin secretion: intracellular energy and calcium dependence of regulated pathway. American journal of physiology. Endocrinology and metabolism, $278(5)$

E892-E901. https://doi.org/10.1152/ajpendo.2000.278.5.E892

Li, B., Cui, W., Tan, Y., Luo, P., Chen, Q., Zhang, C., Qu, W., Miao, L., \& Cai, L. (2014). Zinc is essential for the transcription function of Nrf2 in human renal tubule cells in vitro and mouse kidney in vivo under the diabetic condition. Journal of cellular and molecular medicine, 18(5), 895-906. https://doi.org/10.1111/jcmm.12239

Li, M., Reynolds, C. M., Segovia, S. A., Gray, C., \& Vickers, M. H. (2015). Developmental Programming of Nonalcoholic Fatty Liver Disease: The Effect of Early Life Nutrition on Susceptibility and Disease Severity in Later Life. BioMed research international, 2015, 437107. https://doi.org/10.1155/2015/437107

Liang, Y., Young, J. L., Kong, M., Tong, Y., Qian, Y., Freedman, J. H., \& Cai, L. (2019). Gender Differences in Cardiac Remodeling Induced by a High-Fat Diet and Lifelong, Low-Dose Cadmium Exposure. Chemical research in toxicology, 32(6), https://doi.org/10.1021/acs.chemrestox.8b00386

Liang, T., Zhang, Q., Sun, W., Xin, Y., Zhang, Z., Tan, Y., Zhou, S., Zhang, C., Cai, L., Lu, X., \& Cheng, M. (2015). Zinc treatment prevents type 1 diabetesinduced hepatic oxidative damage, endoplasmic reticulum stress, and cell death, and even prevents possible steatohepatitis in the OVE26 mouse model: Important role of metallothionein. Toxicology letters, 233(2), 114124. https://doi.org/10.1016/i.toxlet.2015.01.010

Lindeque, J. Z., Jansen van Rensburg, P. J., Louw, R., van der Westhuizen, F. H., Florit, S., Ramírez, L., Giralt, M., \& Hidalgo, J. (2015). Obesity and metabolomics: metallothioneins protect against high-fat diet-induced consequences in metallothionein knockout mice. Omics : a journal of integrative biology, 19(2), 92-103. https://doi.org/10.1089/omi.2014.0087

Lionetti, L., Mollica, M. P., Donizzetti, I., Gifuni, G., Sica, R., Pignalosa, A., Cavaliere, G., Gaita, M., De Filippo, C., Zorzano, A., \& Putti, R. (2014). High-lard and high-fish-oil diets differ in their effects on function and dynamic behaviour of rat hepatic mitochondria. PloS one, 9(3), e92753. https://doi.org/10.1371/journal.pone.0092753

Liuzzi, J. P., Aydemir, F., Nam, H., Knutson, M. D., \& Cousins, R. J. (2006). Zip14 (Slc39a14) mediates non-transferrin-bound iron uptake into cells. 
Proceedings of the National Academy of Sciences of the United States of America, 103(37), 13612-13617. https://doi.org/10.1073/pnas.0606424103

Livingstone, C. (2015). Zinc: physiology, deficiency, and parenteral nutrition. Nutrition in clinical practice: official publication of the American Society for Parenteral and Enteral Nutrition, 30(3), 371-382. https://doi.org/10.1177/0884533615570376

Llewellyn, T.O. (1994). Cadmium. (Materials Flow). U.S. Department of Interior, Bureau of Mines, Information Circular 9380. http://pubs.usgs.gov/usbmic/ic9380/cadmium.pdf.

Lonardo, A., Nascimbeni, F., Ballestri, S., Fairweather, D., Win, S., Than, T. A., Abdelmalek, M. F., \& Suzuki, A. (2019). Sex Differences in Nonalcoholic Fatty Liver Disease: State of the Art and Identification of Research Gaps. Hepatology (Baltimore, Md.), 70(4), 1457-1469. https://doi.org/10.1002/hep.30626

Long, M. T., Pedley, A., Massaro, J. M., Hoffmann, U., Ma, J., Loomba, R., Chung, R. T., \& Benjamin, E. J. (2018). A simple clinical model predicts incident hepatic steatosis in a community-based cohort: The Framingham Heart Study. Liver international, 38(8), 1495-1503. https://doi.org/10.1111/liv.13709

Ludwig, J., Viggiano, T. R., McGill, D. B., \& Oh, B. J. (1980). Nonalcoholic steatohepatitis: Mayo Clinic experiences with a hitherto unnamed disease. Mayo Clinic proceedings, 55(7), 434-438.

Martin, E., Smeester, L., Bommarito, P. A., Grace, M. R., Boggess, K., Kuban, K., Karagas, M. R., Marsit, C. J., O'Shea, T. M., \& Fry, R. C. (2017). Sexual epigenetic dimorphism in the human placenta: implications for susceptibility during the prenatal period. Epigenomics, 9(3), 267-278. https://doi.org/10.2217/epi-2016-0132

Martin, E. M., \& Fry, R. C. (2018). Environmental Influences on the Epigenome: Exposure- Associated DNA Methylation in Human Populations. Annual review of public health, 39, 309-333. https://doi.org/10.1146/annurevpublhealth-040617-014629

Mahawar, K. K., Bhasker, A. G., Bindal, V., Graham, Y., Dudeja, U., Lakdawala, M., \& Small, P. K. (2017). Zinc Deficiency after Gastric Bypass for Morbid Obesity: a Systematic Review. Obesity surgery, 27(2), 522-529. https://doi.org/10.1007/s11695-016-2474-8

Luo, M., Luo, P., Zhang, Z., Payne, K., Watson, S., Wu, H., Tan, Y., Ding, Y., Sun, W., Yin, X., Zhang, X., Liu, G., Wintergerst, K., Miao, L., \& Cai, L. (2016). 
Zinc delays the progression of obesity-related glomerulopathy in mice via down-regulating P38 MAPK-mediated inflammation. Obesity (Silver Spring, Md.), 24(6), 1244-1256. https://doi.org/10.1002/oby.21463

Manco M. (2017). Insulin Resistance and NAFLD: A Dangerous Liaison beyond the Genetics. Children (Basel, Switzerland), 4(8), 74. https://doi.org/10.3390/children4080074

Margoshes, M., \& Vallee, B.L. (1957). A cadmium protein from equine kidney cortex. Journal of the American Chemical Society.79, 1813-1814.

Martelli, A., Rousselet, E., Dycke, C., Bouron, A., \& Moulis, J. M. (2006). Cadmium toxicity in animal cells by interference with essential metals. Biochimie, 88(11), 1807-1814. https://doi.org/10.1016/j.biochi.2006.05.013

Martorell, I., Perelló, G., Martí-Cid, R., Llobet, J. M., Castell, V., \& Domingo, J. L. (2011). Human exposure to arsenic, cadmium, mercury, and lead from foods in Catalonia, Spain: temporal trend. Biological trace element research, 142(3), 309-322. https://doi.org/10.1007/s12011-010-8787-x

Mesnage, R., Defarge, N., Rocque, L. M., Spiroux de Vendômois, J., \& Séralini, G. E. (2015). Laboratory Rodent Diets Contain Toxic Levels of Environmental Contaminants: Implications for Regulatory Tests. PloS one, 10(7), e0128429. https://doi.org/10.1371/journal.pone.0128429

Miao, X., Sun, W., Fu, Y., Miao, L., \& Cai, L. (2013). Zinc homeostasis in the metabolic syndrome and diabetes. Frontiers of medicine, 7(1), 31-52. https://doi.org/10.1007/s11684-013-0251-9/

Min, K. S., Ueda, H., \& Tanaka, K. (2008). Involvement of intestinal calcium transporter 1 and metallothionein in cadmium accumulation in the liver and kidney of mice fed a low-calcium diet. Toxicology letters, 176(1), 85-92. https://doi.org/10.1016/j.toxlet.2007.10.011.

Mohanty, A. F., Farin, F. M., Bammler, T. K., MacDonald, J. W., Afsharinejad, Z., Burbacher, T. M., Siscovick, D. S., Williams, M. A., \& Enquobahrie, D. A. (2015). Infant sex-specific placental cadmium and DNA methylation associations. Environmental research, 138, 74-81. https://doi.org/10.1016/i.envres.2015.02.004

Moslehi, A., \& Hamidi-Zad, Z. (2018). Role of SREBPs in Liver Diseases: A Minireview. Journal of clinical and translational hepatology, 6(3), 332-338. https://doi.org/10.14218/JCTH.2017.00061

Namdarghanbari, M. A., Bertling, J., Krezoski, S., \& Petering, D. H. (2014). Toxic metal proteomics: reaction of the mammalian zinc proteome with $\mathrm{Cd}^{2+}$. 
Journal of inorganic biochemistry, 136, 115-121. https://doi.org/10.1016/j.jinorgbio.2014.01.014

Nasiadek, M., Danilewicz, M., Sitarek, K., Świątkowska, E., Daragó, A., Stragierowicz, J., \& Kilanowicz, A. (2018). The effect of repeated cadmium oral exposure on the level of sex hormones, estrous cyclicity, and endometrium morphometry in female rats. Environmental science and pollution research international, 25(28), 28025-28038. https://doi.org/10.1007/s11356-018-2821-5

Nawrot, T. S., Staessen, J. A., Roels, H. A., Munters, E., Cuypers, A., Richart, T., Ruttens, A., Smeets, K., Clijsters, H., \& Vangronsveld, J. (2010). Cadmium exposure in the population: from health risks to strategies of prevention. Biometals, 23(5), 769-782. https://doi.org/10.1007/s10534-010-9343-z

Newbold, R. R., \& McLachlan, J. A. (1996). Transplacental hormonal carcinogenesis: diethylstilbestrol as an example. Progress in clinical and biological research, 394, 131-147.

Nie, X., Wang, N., Chen, Y., Chen, C., Han, B., Zhu, C., Chen, Y., Xia, F., Cang, Z., Lu, M., Meng, Y., Jiang, B., D Jensen, M., \& Lu, Y. (2016). Blood cadmium in Chinese adults and its relationships with diabetes and obesity. Environmental science and pollution research international, 23(18), 1871418723. https://doi.org/10.1007/s11356-016-7078-2

Nogawa, K., \& Kido, T. (1996). Itai-itai disease and health effects of cadmium Chang LW, Toxicology of metals. New York: CRCpress, 353-369.

Norheim, F., Hui, S. T., Kulahcioglu, E., Mehrabian, M., Cantor, R. M., Pan, C., Parks, B. W., \& Lusis, A. J. (2017). Genetic and hormonal control of hepatic steatosis in female and male mice. Journal of lipid research, 58(1), 178187. https://doi.org/10.1194/ilr.M071522

Ogawa, T., Kobayashi, E., Okubo, Y., Suwazono, Y., Kido, T., \& Nogawa, K. (2004). Relationship among prevalence of patients with Itai-itai disease, prevalence of abnormal urinary findings, and cadmium concentrations in rice of individual hamlets in the Jinzu River basin, Toyama prefecture of Japan. International journal of environmental health research, 14(4), 243252. https://doi.org/10.1080/09603120410001725586

Ogden, C.L., Carroll, M.D., Kit, B.K., Flegal, K.M. (2012). Prevalence of obesity in the United States, 2009-2010. NCHS Data Brief, No. 82. National Center for Health Statistics.

Olechnowicz, J., Tinkov, A., Skalny, A., \& Suliburska, J. (2018). Zinc status is associated with inflammation, oxidative stress, lipid, and glucose 
metabolism. The journal of physiological sciences : JPS, 68(1), 19-31. https://doi.org/10.1007/s12576-017-0571-7

Oosterveer, M. H., van Dijk, T. H., Tietge, U. J., Boer, T., Havinga, R., Stellaard, F., Groen, A. K., Kuipers, F., \& Reijngoud, D. J. (2009). High fat feeding induces hepatic fatty acid elongation in mice. PloS one, 4(6), e6066. https://doi.org/10.1371/journal.pone.0006066

Padilla, M. A., Elobeid, M., Ruden, D. M., \& Allison, D. B. (2010). An examination of the association of selected toxic metals with total and central obesity indices: NHANES 99-02. International journal of environmental research and public health, 7(9), 3332-3347. https://doi.org/10.3390/ijerph7093332

Painter, R. C., Roseboom, T. J., \& Bleker, O. P. (2005). Prenatal exposure to the Dutch famine and disease in later life: an overview. Reproductive toxicology (Elmsford, N.Y.), 20(3), 345-352. https://doi.org/10.1016/j.reprotox.2005.04.005

Palmer, B. F., \& Clegg, D. J. (2015). The sexual dimorphism of obesity. Molecular and cellular endocrinology, 402, 113-119. https://doi.org/10.1016/j.mce.2014.11.029

Park, S., Kim, Y. W., Kim, J. Y., Jang, E. C., Doh, K. O., \& Lee, S. K. (2001). Effect of high fat diet on insulin resistance: dietary fat versus visceral fat mass. Journal of Korean medical science, 16(4), 386-390. https://doi.org/10.3346/jkms.2001.16.4.386

Park, W. J., Park, J. W., Merrill, A. H., Storch, J., Pewzner-Jung, Y., \& Futerman, A. H. (2014). Hepatic fatty acid uptake is regulated by the sphingolipid acyl chain length. Biochimica et biophysica acta, 1841(12), 1754-1766. https://doi.org/10.1016/j.bbalip.2014.09.009

Pei, K., Gui, T., Kan, D., Feng, H., Jin, Y., Yang, Y., Zhang, Q., Du, Z., Gai, Z., Wu, J., \& Li, Y. (2020). An Overview of Lipid Metabolism and Nonalcoholic Fatty Liver Disease. BioMed research international, 2020, 4020249. https://doi.org/10.1155/2020/4020249

Pettersson, U. S., Waldén, T. B., Carlsson, P. O., Jansson, L., \& Phillipson, M. (2012). Female mice are protected against high-fat diet induced metabolic syndrome and increase the regulatory $\mathrm{T}$ cell population in adipose tissue. PloS one, 7(9), e46057. https://doi.org/10.1371/journal.pone.0046057

Ponti, F., Plazzi, A., Guglielmi, G., Marchesini, G., \& Bazzocchi, A. (2019). Body composition, dual-energy $\mathrm{X}$-ray absorptiometry and obesity: the paradigm of fat (re)distribution. BJR case reports, 5(3), 20170078. https://doi.org/10.1259/bjrcr.20170078 
Popkin, B. M., Keyou, G., Zhai, F., Guo, X., Ma, H., \& Zohoori, N. (1993). The nutrition transition in China: a cross-sectional analysis. European journal of clinical nutrition, 47(5), 333-346.

Poston, W. S., 2nd, \& Foreyt, J. P. (1999). Obesity is an environmental issue. Atherosclerosis, 146(2), 201-209. https://doi.org/10.1016/s0021$\underline{\text { 9150(99)00258-0 }}$

Qi, Y., Zhang, Z., Liu, S., Aluo, Z., Zhang, L., Yu, L., Li, Y., Song, Z., \& Zhou, L. (2020). Zinc Supplementation Alleviates Lipid and Glucose Metabolic Disorders Induced by a High-Fat Diet. Journal of agricultural and food chemistry, 68(18), 5189-5200. https://doi.org/10.1021/acs.jafc.0c01103

Rao, M., Acharya, Y., Naik, J., Fatteh, ., Fateh, A., Pawar, A., Jayalakshmi, G., Sandhya, B., \& Arja, Sireesha Bala. (2017). Effect of Heavy Metals on the Activity Levels of Hepatic Enzymes in the Maternal and Embryonic Tissue of Viviparous Scorpion (H. Fulvipes). International Journal of Life-Sciences Scientific Research, 3. 10.21276/ijlssr.2017.3.6.3.

Ravelli, A. C. J., van der Meulen, J. H. P., Osmond, C., Barker, D. J.P., and Bleker, O. P. (1999). Obesity at the age of $50 \mathrm{y}$ in men and women exposed to famine prenatally. American Journal of Clinical Nutrition, 70, 811-816.

Recena Aydos, L., Aparecida do Amaral, L., Serafim de Souza, R., Jacobowski, A. C., Freitas Dos Santos, E., \& Rodrigues Macedo, M. L. (2019). Nonalcoholic Fatty Liver Disease Induced by High-Fat Diet in C57bl/6 Models. Nutrients, 11(12), 3067. https://doi.org/10.3390/nu11123067.

Riant, E., Waget, A., Cogo, H., Arnal, J. F., Burcelin, R., \& Gourdy, P. (2009). Estrogens protect against high-fat diet-induced insulin resistance and glucose intolerance in mice. Endocrinology, 150(5), 2109-2117. https://doi.org/10.1210/en.2008-0971

Ricci, M., \& Ulman, E. (2005). Laboratory animal diets: A critical part of your in vivo research. Animal Laboratory News, 4(6), 1-6.

Rinella, M. E. (2015). Nonalcoholic fatty liver disease: a systematic review. JAMA, 313(22), 2263-2273. https://doi.org/10.1001/jama.2015.5370

Roberts, S. B., \& Rosenberg, I. (2006). Nutrition and aging: changes in the regulation of energy metabolism with aging. Physiological reviews, 86(2), 651-667. https://doi.org/10.1152/physrev.00019.2005

Roseboom, T. J., van der Meulen, J. H., Osmond, C., Barker, D. J., Ravelli, A. C., Schroeder-Tanka, J. M., van Montfrans, G. A., Michels, R. P., \& Bleker, O. 
P. (2000). Coronary heart disease after prenatal exposure to the Dutch famine, 1944-45. Heart (British Cardiac Society), 84(6), 595-598. https://doi.org/10.1136/heart.84.6.595

Ruhl, C. E., \& Everhart, J. E. (2015). Fatty liver indices in the multiethnic United States National Health and Nutrition Examination Survey. Alimentary pharmacology \& therapeutics, 41(1), 65-76. https://doi.org/10.1111/apt.13012

Ryu, D. Y., Lee, S. J., Park, D. W., Choi, B. S., Klaassen, C. D., \& Park, J. D. (2004). Dietary iron regulates intestinal cadmium absorption through iron transporters in rats. Toxicology letters, 152(1), 19-25. https://doi.org/10.1016/i.toxlet.2004.03.015

Sakulsak, N. (2012). Metallothionein: an overview on its metal homeostatic regulation in mammals. International Journal of Morphology, 30(3), 10071012.

Salinero, A. E., Anderson, B. M., \& Zuloaga, K. L. (2018). Sex differences in the metabolic effects of diet-induced obesity vary by age of onset. International journal of obesity (2005), 42(5), 1088-1091. https://doi.org/10.1038/s41366-018-0023-3

Sanyaolu, A., Okorie, C., Qi, X., Locke, J., \& Rehman, S. (2019). Childhood and Adolescent Obesity in the United States: A Public Health Concern. Global pediatric health, $6, \quad 2333794 X 19891305$. https://doi.org/10.1177/2333794X19891305

Saponaro, C., Gaggini, M., Carli, F., \& Gastaldelli, A. (2015). The Subtle Balance between Lipolysis and Lipogenesis: A Critical Point in Metabolic Homeostasis. Nutrients, 7(11), 9453-9474. https://doi.org/10.3390/nu7115475

Saris, W. H., Astrup, A., Prentice, A. M., Zunft, H. J., Formiguera, X., Verboeketvan de Venne, W. P., Raben, A., Poppitt, S. D., Seppelt, B., Johnston, S., Vasilaras, T. H., \& Keogh, G. F. (2000). Randomized controlled trial of changes in dietary carbohydrate/fat ratio and simple vs complex carbohydrates on body weight and blood lipids: the CARMEN study. The Carbohydrate Ratio Management in European National diets. International journal of obesity and related metabolic disorders, 24(10), 1310-1318. https://doi.org/10.1038/sj.ijo.0801451

Satapati, S., Sunny, N. E., Kucejova, B., Fu, X., He, T. T., Méndez-Lucas, A., Shelton, J. M., Perales, J. C., Browning, J. D., \& Burgess, S. C. (2012). Elevated TCA cycle function in the pathology of diet-induced hepatic insulin 
resistance and fatty liver. Journal of lipid research, 53(6), 1080-1092. https://doi.org/10.1194/jlr.M023382

Sato, M., Kawakami, T., Kadota, Y., Mori, M., \& Suzuki, S. (2013). Obesity and metallothionein. Current pharmaceutical biotechnology, 14(4), 432-440. https://doi.org/10.2174/1389201011314040008

Seo, J. A., Song, S. W., Han, K., Lee, K. J., \& Kim, H. N. (2014). The associations between serum zinc levels and metabolic syndrome in the Korean population: findings from the 2010 Korean National Health and Nutrition Examination Survey. PloS one, 9(8), e105990. https://doi.org/10.1371/journal.pone.0105990

Shidfar, F., Faghihi, A., Amiri, H. L., \& Mousavi, S. N. (2018). Regression of Nonalcoholic Fatty Liver Disease with Zinc and Selenium Cosupplementation after Disease Progression in Rats. Iranian journal of medical sciences, 43(1), 26-31.

Shimano, H., Horton, J. D., Shimomura, I., Hammer, R. E., Brown, M. S., \& Goldstein, J. L. (1997). Isoform 1c of sterol regulatory element binding protein is less active than isoform 1a in livers of transgenic mice and in cultured cells. The Journal of clinical investigation, 99(5), 846-854. https://doi.org/10.1172/JCl119248

Simmons, A. L., Schlezinger, J. J., \& Corkey, B. E. (2014). What Are We Putting in Our Food That Is Making Us Fat? Food Additives, Contaminants, and Other Putative Contributors to Obesity. Current obesity reports, 3(2), 273285. https://doi.org/10.1007/s13679-014-0094-y

Sinaiko, A. R., Steinberger, J., Moran, A., Prineas, R. J., Vessby, B., Basu, S., Tracy, R., \& Jacobs, D. R., Jr (2005). Relation of body mass index and insulin resistance to cardiovascular risk factors, inflammatory factors, and oxidative stress during adolescence. Circulation, 111(15), 1985-1991. https://doi.org/10.1161/01.CIR.0000161837.23846.57

Smilowitz, J.T., German, J.B., Zivkovic, A.M. Food Intake and Obesity: The Case of Fat. In: Montmayeur JP, le Coutre J, editors. Fat Detection: Taste, Texture, and Post Ingestive Effects. Boca Raton (FL): CRC Press/Taylor \& Francis; $2010 . \quad$ Chapter $22 . \quad$ Available from: https://www.ncbi.nlm.nih.gov/books/NBK53555/

Spruss, A., Henkel, J., Kanuri, G., Blank, D., Püschel, G. P., Bischoff, S. C., \& Bergheim, I. (2012). Female mice are more susceptible to nonalcoholic fatty liver disease: sex-specific regulation of the hepatic AMP-activated protein kinase-plasminogen activator inhibitor 1 cascade, but not the hepatic 
endotoxin response. Molecular medicine (Cambridge, Mass.), 18(1), 13461355. https://doi.org/10.2119/molmed.2012.00223

Strakovsky, R. S., Zhang, X., Zhou, D., \& Pan, Y. X. (2014). The regulation of hepatic Pon 1 by a maternal high-fat diet is gender specific and may occur through promoter histone modifications in neonatal rats. The Journal of nutritional biochemistry, 25(2), 170-176. https://doi.org/10.1016/i.jnutbio.2013.09.016

Stonard, M. D., \& Webb, M. (1976). Influence of dietary cadmium on the distribution of the essential metals copper, zinc and iron in tissues of the rat. Chemico-biological interactions, 15(4), 349-363. https://doi.org/10.1016/0009-2797(76)90140-x

Suliburska, J., Cofta, S., Gajewska, E., Kalmus, G., Sobieska, M., Samborski, W., Krejpcio, Z., Drzymala-Czyz, S., \& Bogdanski, P. (2013). The evaluation of selected serum mineral concentrations and their association with insulin resistance in obese adolescents. European review for medical and pharmacological sciences, 17(17), 2396-2400.

Supplee, W.C. (1961). Production of zinc deficiency in turkey poults by dietary cadmium. Poultry Science, 40:827

Targher, G., Day, C. P., \& Bonora, E. (2010). Risk of cardiovascular disease in patients with nonalcoholic fatty liver disease. The New England journal of medicine, 363(14), 1341-1350. https://doi.org/10.1056/NEJMra0912063

Tellez-Plaza, M., Guallar, E., Howard, B. V., Umans, J. G., Francesconi, K. A., Goessler, W., Silbergeld, E. K., Devereux, R. B., \& Navas-Acien, A. (2013). Cadmium exposure and incident cardiovascular disease. Epidemiology (Cambridge, Mass.), 24(3), 421-429. https://doi.org/10.1097/EDE.0b013e31828b0631

Thoen, R. U., Barther, N. N., Schemitt, E., Bona, S., Fernandes, S., Coral, G., Marroni, N. P., Tovo, C., Guedes, R. P., \& Porawski, M. (2019). Zinc supplementation reduces diet-induced obesity and improves insulin sensitivity in rats. Applied physiology, nutrition, and metabolism = Physiologie appliquee, nutrition et metabolisme, 44(6), 580-586. https://doi.org/10.1139/apnm-2018-0519

Tinkov, A. A., Filippini, T., Ajsuvakova, O. P., Aaseth, J., Gluhcheva, Y. G., Ivanova, J. M., Bjørklund, G., Skalnaya, M. G., Gatiatulina, E. R., Popova, E. V., Nemereshina, O. N., Vinceti, M., \& Skalny, A. V. (2017). The role of cadmium in obesity and diabetes. The Science of the total environment, 601-602, 741-755. https://doi.org/10.1016/j.scitotenv.2017.05.224 
Tramunt, B., Smati, S., Grandgeorge, N., Lenfant, F., Arnal, J. F., Montagner, A., \& Gourdy, P. (2020). Sex differences in metabolic regulation and diabetes susceptibility. Diabetologia, 63(3), 453-461. https://doi.org/10.1007/s00125-019-05040-3

Tucker, L. A., \& Kano, M. J. (1992). Dietary fat and body fat: a multivariate study of 205 adult females. The American journal of clinical nutrition, 56(4), 616622. https://doi.org/10.1093/ajcn/56.4.616

Türkcan, A., Scharinger, B., Grabmann, G., Keppler, B. K., Laufer, G., Bernhard, D., \& Messner, B. (2015). Combination of cadmium and high cholesterol levels as a risk factor for heart fibrosis. Toxicological sciences, 145(2), 360371. https://doi.org/10.1093/toxsci/kfv057

van Abeelen, A. F., Elias, S. G., Bossuyt, P. M., Grobbee, D. E., van der Schouw, Y. T., Roseboom, T. J., \& Uiterwaal, C. S. (2012). Famine exposure in the young and the risk of type 2 diabetes in adulthood. Diabetes, 61(9), 22552260. https://doi.org/10.2337/db11-1559

Vacchi-Suzzi, C., Kruse, D., Harrington, J., Levine, K., \& Meliker, J. R. (2016). Is Urinary Cadmium a Biomarker of Long-term Exposure in Humans? A Review. Current environmental health reports, 3(4), 450-458. https://doi.org/10.1007/s40572-016-0107-y

Vahter, M., Åkesson, A., Lidén, C., Ceccatelli, S., Berglund, M., 2007. Gender differences in the disposition and toxicity of metals. Environ. Res. 104 (1), 85-95. https://doi.org/10.1016/j.envres.2006.08.003.

Velázquez, K. T., Enos, R. T., Bader, J. E., Sougiannis, A. T., Carson, M. S., Chatzistamou, I., Carson, J. A., Nagarkatti, P. S., Nagarkatti, M., \& Murphy, E. A. (2019). Prolonged high-fat-diet feeding promotes non-alcoholic fatty liver disease and alters gut microbiota in mice. World journal of hepatology, 11(8), 619-637. https://doi.org/10.4254/wih.v11.i8.619

Vesey D. A. (2010). Transport pathways for cadmium in the intestine and kidney proximal tubule: focus on the interaction with essential metals. Toxicology letters, 198(1), 13-19. https://doi.org/10.1016/j.toxlet.2010.05.004

Via, M. (2012). The malnutrition of obesity: micronutrient deficiencies that promote diabetes. ISRN endocrinology, 2012, 103472. https://doi.org/10.5402/2012/103472

Waalkes, M. P., Kovatch, R., \& Rehm, S. (1991). Effect of chronic dietary zinc deficiency on cadmium toxicity and carcinogenesis in the male Wistar [Hsd: (WI)BR] rat. Toxicology and applied pharmacology, 108(3), 448-456. https://doi.org/10.1016/0041-008x(91)90091-r 
Waalkes, M. P., Ward, J. M., Liu, J., \& Diwan, B. A. (2003). Transplacental carcinogenicity of inorganic arsenic in the drinking water: induction of hepatic, ovarian, pulmonary, and adrenal tumors in mice. Toxicology and applied pharmacology, 186(1), 7-17. https://doi.org/10.1016/s0041008x(02)00022-4

Wang, H., Zhu, G., Shi, Y., Weng, S., Jin, T., Kong, Q., \& Nordberg, G. F. (2003). Influence of environmental cadmium exposure on forearm bone density. Journal of bone and mineral research: the official journal of the American Society for Bone and Mineral Research, 18(3), 553-560. https://doi.org/10.1359/jbmr.2003.18.3.553

Wang, S., Luo, M., Zhang, Z., Gu, J., Chen, J., Payne, K. M., Tan, Y., Wang, Y., Yin, X., Zhang, X., Liu, G. C., Wintergerst, K., Liu, Q., Zheng, Y., \& Cai, L. (2016). Zinc deficiency exacerbates while zinc supplement attenuates cardiac hypertrophy in high-fat diet-induced obese mice through modulating p38 MAPK-dependent signaling. Toxicology letters, 258, 134-146. https://doi.org/10.1016/..toxlet.2016.06.020

Wang, S., Gu, J., Xu, Z., Zhang, Z., Bai, T., Xu, J., Cai, J., Barnes, G., Liu, Q. J., Freedman, J. H., Wang, Y., Liu, Q., Zheng, Y., \& Cai, L. (2017). Zinc rescues obesity-induced cardiac hypertrophy via stimulating metallothionein to suppress oxidative stress-activated BCL10/CARD9/p38 MAPK pathway. Journal of cellular and molecular medicine, 21(6), 11821192. https://doi.org/10.1111/jcmm. 13050

Welsh, J. A., Karpen, S., \& Vos, M. B. (2013). Increasing prevalence of nonalcoholic fatty liver disease among United States adolescents, 19881994 to 2007-2010. The Journal of pediatrics, 162(3), 496-500.e1. https://doi.org/10.1016/.jpeds.2012.08.043

Williams, C. D., Stengel, J., Asike, M. I., Torres, D. M., Shaw, J., Contreras, M., Landt, C. L., \& Harrison, S. A. (2011). Prevalence of nonalcoholic fatty liver disease and nonalcoholic steatohepatitis among a largely middle-aged population utilizing ultrasound and liver biopsy: a prospective study. Gastroenterology, 140(1), https://doi.org/10.1053/i.gastro.2010.09.038

Winzell, M. S., \& Ahrén, B. (2004). The high-fat diet-fed mouse: a model for studying mechanisms and treatment of impaired glucose tolerance and type 2 diabetes. Diabetes, 53 Suppl 3, S215-S219. https://doi.org/10.2337/diabetes.53.suppl 3.s215

Wu, C., Zhang, Y., Chai, L., \& Wang, H. (2017). Histological changes, lipid metabolism and oxidative stress in the liver of Bufo gargarizans exposed to 
cadmium concentrations. Chemosphere, 179, 337-346. https://doi.org/10.1016/j.chemosphere.2017.03.131

Xiaoping, Z., \& Fajun, Y. (2012). Regulation of SREBP-Mediated Gene Expression. Sheng Wu Wu Li Hsueh Bao, 28(4), 287-294. https://doi.org/10.3724/SP.J.1260.2012.20034

Young, J. L., \& Cai, L. (2020). Implications for prenatal cadmium exposure and adverse health outcomes in adulthood. Toxicology and applied pharmacology, 403, 115161. https://doi.org/10.1016/.t.taap.2020.115161

Young, J. L., Yan, X., Xu, J., Yin, X., Zhang, X., Arteel, G. E., Barnes, G. N., States, J. C., Watson, W. H., Kong, M., Cai, L., \& Freedman, J. H. (2019). Cadmium and High-Fat Diet Disrupt Renal, Cardiac and Hepatic Essential Metals. Scientific reports, 9(1), 14675. https://doi.org/10.1038/s41598-019-50771-3

Younossi, Z., Anstee, Q. M., Marietti, M., Hardy, T., Henry, L., Eslam, M., George, J., \& Bugianesi, E. (2018). Global burden of NAFLD and NASH: trends, predictions, risk factors and prevention. Nature reviews. Gastroenterology \& hepatology, 15(1), 11-20. https://doi.org/10.1038/nrgastro.2017.109

Younossi, Z. M., Blissett, D., Blissett, R., Henry, L., Stepanova, M., Younossi, Y., Racila, A., Hunt, S., \& Beckerman, R. (2016). The economic and clinical burden of nonalcoholic fatty liver disease in the United States and Europe. Hepatology (Baltimore, Md.), 64(5), 1577-1586. https://doi.org/10.1002/hep.28785

Zhai, Q., Narbad, A., \& Chen, W. (2015). Dietary strategies for the treatment of cadmium and lead toxicity. Nutrients, 7(1), 552-571. https://doi.org/10.3390/nu7010552

Zhang, D., Liu, J., Gao, J., Shahzad, M., Han, Z., Wang, Z., Li, J., \& Sjölinder, H. (2014). Zinc supplementation protects against cadmium accumulation and cytotoxicity in Madin-Darby bovine kidney cells. PloS one, 9(8), e103427. https://doi.org/10.1371/journal.pone.0103427

Zhang, H., \& Cai, L. (2020). Zinc homeostasis plays an important role in the prevention of obesity-induced cardiac inflammation, remodeling and dysfunction. Journal of trace elements in medicine and biology: organ of the Society for Minerals and Trace Elements (GMS), 62, 126615. Advance online publication. https://doi.org/10.1016/.j.jemb.2020.12

Zhang, J., Wang, Y., Fu, L., Feng, Y. J., Ji, Y. L., Wang, H., \& Xu, D. X. (2018). Subchronic cadmium exposure upregulates the mRNA level of genes associated to hepatic lipid metabolism in adult female CD1 mice. Journal of applied toxicology : JAT, 38(7), 1026-1035. https://doi.org/10.1002/jat.3612 
Zhang, S., Jin, Y., Zeng, Z., Liu, Z., \& Fu, Z. (2015). Subchronic Exposure of Mice to Cadmium Perturbs Their Hepatic Energy Metabolism and Gut Microbiome. Chemical research in toxicology, 28(10), 2000-2009. https://doi.org/10.1021/acs.chemrestox.5b0023 
APPENDIX I

Chapters 1, 3 and 4 of this dissertation contain modified content from Young and Cai, 2020 and Young et al., 2018. Rights for use are below in captured screen shots.

\begin{tabular}{|c|c|c|c|c|c|}
\hline $8 \begin{array}{l}\text { Copyright } \\
\text { Clearance }\end{array}$ & RightsLink & $\underset{\text { Home }}{\hat{\mathbf{A}}}$ & $\begin{array}{c}? \\
\text { Help }\end{array}$ & $\underset{\text { Email Support }}{\boldsymbol{\square}}$ & $\underset{\text { Jamie Young } v}{2}$ \\
\hline 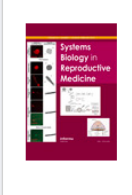 & $\begin{array}{l}\text { Impact of prenatal arsenic exposure on chronic } \\
\text { Author: Jamie L. Young, , Lu Cai, et al } \\
\text { Publication: SYSTEMS BIOLOGY IN REPRODUCTIVE MEDICINE } \\
\text { Publisher: Taylor \& Francis } \\
\text { Date: Nov 2, } 2018 \\
\text { Rights managed by Taylor \& Francis }\end{array}$ & & & & \\
\hline $\begin{array}{l}\text { Thesis/Disse } \\
\text { Taylor \& Franc } \\
\text { BACK }\end{array}$ & $\begin{array}{l}\text { rtation Reuse Request } \\
\text { is is pleased to offer reuses of its content for a thesis or disserta }\end{array}$ & ission re & uest if $\mathrm{W}$ & rk is published. & CLOSE \\
\hline
\end{tabular}

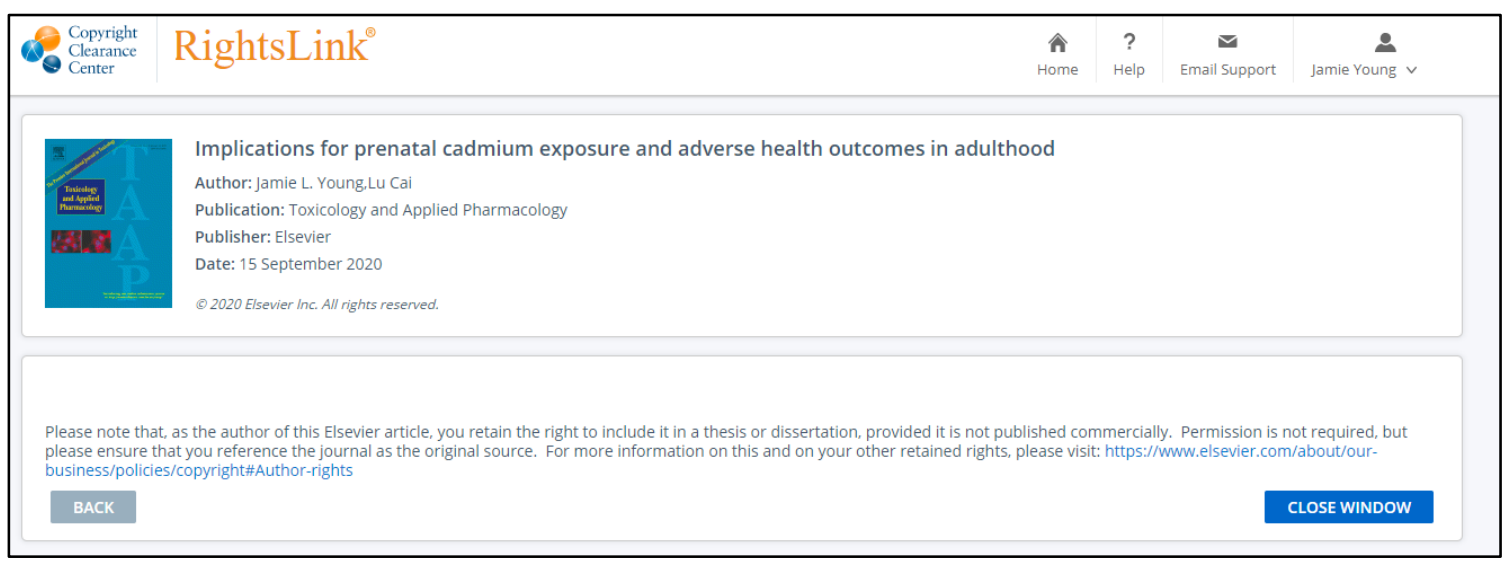




\section{LIST OF ABBREVIATIONS}

\begin{tabular}{ll} 
ALT & Alanine aminotransferase \\
AST & Aspartate aminotransferase \\
AUC & Area under the curve \\
BMC & Bone mineral content \\
BMD & Bone mineral density \\
CaT1 & Channel-like calcium transport \\
Cd & Cadmium \\
CDNA & Complementary DNA \\
CVD & Cardiovascular disease \\
DEXA & Dual energy X-ray absorptiometry \\
DMT-1 & Divalent metal transporter 1 \\
DNA & Deoxyribonucleic acid \\
FASN & Fatty acid synthase \\
GAPDH & Glyceraldehyde 3-phosphate dehydrogenase \\
GSH & Glutathione \\
H\&E & Hematoxylin \& eosin \\
HFD & High fat diet \\
IPGTT & Intraperitoneal glucose tolerance test \\
\hline
\end{tabular}




\begin{tabular}{ll} 
mRNA & Messenger RNA \\
MT & Metallothionein \\
MTP1 & Metal transporter 1 \\
NAFL & Nonalcoholic fatty liver \\
NAFLD & Nonalcoholic fatty liver disease \\
NASH & Nonalcoholic steatohepatitis \\
ND & Normal Diet \\
NPL & National Priority List \\
ORO & Oil red O \\
PPB & parts per billion \\
PPM & parts per million \\
SCD1 & Stearoyl coenzyme-A desaturase1 \\
SD & Standard Deviation \\
SEM & Standard error of the mean \\
SREBP & Sterol regulatory element-binding protein \\
ZIP & Zrt- and Irt-like protein \\
Zn & Zinc \\
\hline
\end{tabular}




\section{CURRICULUM VITAE}

Jamie Lynn Young

Mailing Address: Department of Pharmacology and Toxicology, School of Medicine University of Louisville 505 South Hancock Street Louisville, KY 40292

Email:_ jlyoun16@louisville.edu

Phone: $\quad$ 207-808-3882

\section{Education}

2003-2007 B.A. in Biology: cum laude, University of Maine at Farmington

2015-2018 M.S. in Pharmacology and Toxicology, University of Louisville

\section{Current Appointments}

Doctoral Student, Department of Pharmacology and Toxicology, University of Louisville

Student Representative, Ohio Valley Society of Toxicology

Student Member, Professional Development Subcommittee, Society of Toxicology Graduate Student Leadership Committee

Student Representative, University of Louisville NIEHS Superfund Research Center Training Core

\section{Professional Experience}

2018-Present Doctoral Student, Department of Pharmacology and Toxicology, University of Louisville, Louisville, KY

2015-2018 Masters Student, Department of Pharmacology and Toxicology, University of Louisville, Louisville, KY 
2015 Research Associate, Wise Laboratory of Environmental and Genetic Toxicology, University of Southern Maine, Portland, ME

2010-2015 Environmental Scientist, Portland Water District, Portland, ME

2010 Analytical Chemist, Katahdin Analytical Services, Scarborough, ME

2007-2010 Doctoral Student, Wise Laboratory of Environmental and Genetic Toxicology, University of Southern Maine, Portland, ME.

2005-2007 Research Assistant III, Dr. Ronald Butler - Spatial Ecology Laboratory, University of Maine at Farmington, Farmington, ME.

2005 Research Assistant III, The Jackson Laboratory-Genotyping Lab, Bar Harbor, ME.

Fall 2005 Tutor, Anatomy and Physiology, University of Maine at Farmington, Farmington, ME

\section{Professional Appointments}

2019-Present Student Representative, Ohio Valley Society of Toxicology (OVSOT)

2019-Present Member, Professional Development Subcommittee, Society of Toxicology Graduate Student Leadership Committee

2019-Present Student Representative, University of Louisville NIEHS Superfund Research Center Training Core

2019-2020 Chair, Society of Toxicology (SOT) Graduate Student Leadership Committee

2019 Member, NIEHS Superfund Research Program Trainee Session Planning Committee

2018-2019 Chair, Programming Subcommittee, Society of Toxicology (SOT) Graduate Student Leadership Committee

2017-2018 Secretary, Programming Subcommittee, Society of Toxicology (SOT) Graduate Student Leadership Committee

2016-2018 Student Representative, SOT Metals Specialty Section Graduate 


\section{Seminars/Platform sessions}

2021 Symposium, "Sexual Dimorphism of Cadmium-Exacerbated, Diet-Induced Liver Disease". Society of Toxicology 2021 Virtual Meeting. To be presented virtually, March 2021

2020 Research Seminar, "The Effects of Whole Life, Low Dose Cadmium Exposure on High Fat Diet-induced NAFLD and the Role of Zinc". Department of Pharmacology and Toxicology Seminar, University of Louisville, Louisville, KY. September, 2020.

2019 Research Seminar, "The Effects of Whole Life, Low Dose Cadmium Exposure on High Fat Diet-induced NAFLD and the Role of Zinc". Center for Neuroscience and Cell Biology, University of Coimbra, Portugal. May 2019.

2018 Research Seminar, "Rockets, Bombs and Aquatic Reptiles: A One Environmental Health Perspective". PiE MBB Seminars. Research Centre for Experimental Marine Biology \& Biotechnology Plentzia Marine Station University of the Basque Country (PIE-UPV/EHU) Areatza, Plentzia-Bizkaia E-48620, Basque Country (Spain). September 2018.

Research Seminar, "Whole Life Exposure to Cadmium and Its Influence on Diet-Induced Liver Damage". Work in Progress Seminar, Department of Pediatrics, University of Louisville, Louisville, KY. January, 2018.

2016 Platform Session, "One Environmental Health Perspective: Insight into the Cytotoxic and Genotoxic Effects of Hexavalent Chromium in Human, Aquatic Reptile and Aquatic Mammal Skin Cells" $47^{\text {th }}$ Annual Meeting of the International Association for Aquatic and Animal Medicine, Virginia Beach, VA. May 2016.

Research Seminar, "Exploring the Impact of Chromium Exposure on Macrophage Polarization: Implications for the Innate Immune Response". University of Louisville, Louisville, KY. April 2016.

2009 Platform Session, "Zinc Chromate Induced Spindle Assembly Checkpoint Bypass and Chromosome Instability in Human Lung Cells." $36^{\text {th }}$ Biological and Medical Science Symposium, Mount Desert Biological Laboratory, Bar Harbor, Maine, April 2009.

Seminar, "Zinc Chromate Induced Spindle Assembly Checkpoint Bypass and Chromosome Instability in Human Lung Cells." Department of Applied Medical Science, University of Southern Maine, Portland, Maine, April 2009. 
2008 Seminar. "Biomedical Science Research at the University of Southern Maine." $1^{\text {st }}$ Annual Meeting of the Graduate School of Biomedical Sciences, University of Maine, Orono, May 2008.

Seminar. "Mechanisms of Particulate $\mathrm{Cr}(\mathrm{VI})$ Induced Spindle Assembly Checkpoint Bypass and Chromosome Instability in Human Lung Cells." Department of Applied Medical Science, University of Southern Maine, Portland, Maine, March 2008.

\section{Public Presentations}

2018 Radio Show - "UofL Today" with Mark Hebert. "How do arsenic and cadmium impact human health?

\section{Honors and Awards}

2020 Guy Stevenson Award for Excellence in Graduate Studies, University of Louisville

2020 K.C. Huang Outstanding Graduate Student, Department of Pharmacology and Toxicology, University of Louisville

2020 Ohio Valley Society of Toxicology, 1st place "Tox on the Clock"

2020 Society of Toxicology (SOT) Metals Specialty Section Graduate Student Research Award, First Place

2020 59th Annual Society of Toxicology (SOT) Meeting Graduate Student Travel Award, Anaheim, California

2019 Ohio Valley Society of Toxicology, $1^{\text {st }}$ Place PhD platform Award

2019 13th Conference of the International Society for Trace Element Research in Humans (ISTERH), $1^{\text {st }}$ Place Student Presentation

2019 University of Louisville Nomination for F99/K00 Fellowship

$201810^{\text {th }}$ Conference on Metal Toxicology and Carcinogenesis Student Travel Award, Albuquerque New Mexico

2018 Ohio Valley Society of Toxicology, $1^{\text {st }}$ Place PhD poster

2018 Graduate Deans Citation, University of Louisville

2017 Research!Louisville 2017, $2^{\text {nd }}$ place poster in Masters Basic-Science

2015 Student Travel Award, Environmental Mutagenesis and Genomics Society

2008 Epsilon Pi Tau Honor Society

2007 Michael D. Wilson Scholar, University of Maine at Farmington

2005 Peter Mills Scholarship, University of Maine at Farmington

\section{Grants and Fellowships}

2008-2010 Recipient of the Environmental Protection Agency's Greater Research Opportunities (GRO) Fellowship

2018-2020 NIEHS T32 Predoctoral Fellowships in Environmental Health Sciences

2020 Doctoral Dissertation Completion Award for Fall 2020 


\section{Professional Memberships \& Societies}

Ohio Valley Chapter, Society of Toxicology (OVSOT)

Society of Marine Mammalogy (SMM)

Society of Toxicology (SOT)

American Heart Association (AHA)

International Society for Trace Element Research in Humans (ISTERH)

\section{Workshops/Webinars}

2018 Workshop - Engagement \& Communication Training (Organized by the UofL Superfund Research Center Training Core) August 24, 2018, Louisville, KY

2019 Webinar - "Building Your Village: How to Foster Effective Mentor-Mentee Relationships" (Organized by the Society of Toxicology Graduate Student Leadership Committee), February 20, 2019.

Trainee Program - NIEHS 2019 Superfund Research Program Annual Meeting, November 18, 2019. Event Organizer - "lunch with an expert" session.

2020 Webinar - "Adverse Outcome Pathways" (Organized by The Environmental Mutagenesis and Genomics Society), Wed, Jan 22, 2020.

Webinar - "Best Practices for the Academic Job Hunt" (Organized by the Society of Toxicology Postdoctoral Assembly). Wednesday, January 22, 2020.

Webinar - "How to get the most out of your mentoring relationships" (Organized by the Society of Toxicology Postdoctoral Assembly). Wednesday, February 26, 2020. Invited Moderator

Workshop - Research publishing workshop by David Parsons from Elsevier publishing on how to write a great research paper, find the right journal, and get accepted as well as publishing ethics. April 16 ${ }^{\text {th }}, 2020$.

Webinar - "Exposures and Latent Disease Risk: Session I - Linking Exposures to Diseases with Long Latency Periods" (Hosted by NIEHS Superfund Research Program (SRP). May 11 2020.

Webinar - "Exposures and Latent Disease Risk: Session II - Identifying Hallmarks and Key Characteristics" (Hosted by NIEHS Superfund Research Program (SRP). May 28 ${ }^{\text {th }}, 2020$. 
Webinar - "Exposures and Latent Disease Risk: Session III - Arsenic as a Case Study" (Hosted by NIEHS Superfund Research Program (SRP). June $8^{\text {th }}, 2020$.

Webinar - "Exposures and Latent Disease Risk: Session IV - Moving Forward" (Hosted by NIEHS Superfund Research Program (SRP). June $16^{\text {th }}, 2020$.

Webinar - "Communicating Science in the Misinformation Age: Strategies for Early Career Scientists" (Organized by the Society of Toxicology Postdoctoral Assembly). September 22 ${ }^{\text {nd }}, 2020$.

\section{Teaching}

\section{Individual Lectures}

2019 Lecture on "Anti-neoplastic Drugs" for graduate level course "Dental Hygiene Pharmacology" (Course DHED 402) School of Dentistry, University of Louisville

\section{Meetings Attended}

2008 Annual Meeting for the American Association for Cancer Research

Toxics and Tomorrow's Children - University of Southern Maine

Thinking Matters - University of Southern Maine

2009 Annual Meeting for the Society of Toxicology

Thinking Matters - University of Southern Maine $36^{\text {th }}$ Biological and Medical Science Symposium

Annual Meeting of the Northeast Regional Chapter of the Society of Toxicology

$201554^{\text {th }}$ Annual Meeting for the Society of Toxicology

5th Georgian Bay International Conference on Bioinorganic Chemistry

$46^{\text {th }}$ Annual Meeting of the Environmental Mutagenesis and Genomics Society

Research!Louisville- University of Louisville

$21^{\text {st }}$ Biennial Meeting of the Society of Marine Mammalogy

$201655^{\text {th }}$ Annual Meeting of the Society of Toxicology

$47^{\text {th }}$ Annual Meeting of the International Association for Aquatic and Animal Medicine

Research!Louisville- University of Louisville, October 2016

$201756^{\text {th }}$ Annual Meeting of the Society of Toxicology

Research!Louisville - University of Louisville, September 2017 
Annual Meeting, Ohio Valley Chapter of the Society of Toxicology, December 2017

2018 57th Annual Society of Toxicology Meeting, March 2018 Graduate Student Regional Research Conference, March 2018 Research!Louisville - University of Louisville, October 2018 $10^{\text {th }}$ Conference on Metal Toxicity \& Carcinogenesis, October 2018 Annual Meeting, Ohio Valley Chapter of the Society of Toxicology, November 2018

$201958^{\text {th }}$ Annual Society of Toxicology (SOT) Meeting, Baltimore March 2019 The $15^{\text {th }}$ International Congress of Toxicology (ICTXV), Hawaii July 2019 13th Conference of the International Society for Trace Element Research in Humans (ISTERH) Bali, Indonesia, September 2019 Research!Louisville - University of Louisville, September 2019 Annual Meeting, Ohio Valley Chapter of the Society of Toxicology, October 2019

Annual National Institute of Environmental Health Sciences Superfund Research Program Meeting in Seattle, Washington November 2019.

World Marine Mammal Conference in Barcelona, Spain December 2019

2020 OVSOT Student \& Postdoctoral Researchers' Virtual Summer Meeting July 2020 (Organizer)

\section{Publications}

\section{Journals}

1. Xie, H., Holmes, A.L., Young, J.L., Qin, Q., Joyce, K, Pelsue, S.C., Peng, C., Wise, S.S., Jeevarajan, A., Wallace, W.T., Hammond, D., Wise, Sr., J.P. (2009). Zinc Chromate Induces Chromosome Instability and DNA Double Strand Breaks in Human Lung Cells. Toxicology and Applied Pharmacology. 234:293-299. PMID: 19027772; PMCID: PMC4075174.

2. Holmes, A.L., Wise, S., Pelsue, S., Aboueissa, A., Lingle, W., Salisbury, J., Gallagher, J.L., Wise, Sr., J.P. (2010). Chronic exposure to zinc chromate induces centrosome amplification and spindle assembly checkpoint bypass in human lung fibroblasts. Chemical Research in Toxicology. 23(2):386-395. PMID:20030412

3. Xie, H., Holmes, A.H., Wise, S.S., Young, J.L., Wise, J.T.F. and Wise, Sr., J.P. (2015). Human Skin Cells Are More Sensitive than Human Lung Cells to the Cytotoxic And Cell Cycle Arresting Impacts of Particulate and Soluble Hexavalent Chromium. Biological Trace Element Research. 166(1):49-56. PMID: 25805272. PMCID: PMC4470775. 
4. Young, J.L., Wise, S.S., Xie, H., Zhu, C., Fukuda, T., and Wise, Sr., J.P. (2015). Comparative Cytotoxicity and Genotoxicity of Soluble and Particulate Hexavalent Chromium in Human and Hawksbill Sea Turtle (Eretmochelys imbricate) Skin Cells. Comparative Biochemistry and Physiology. Part C 178 145-155. PMID: 26440299. PMCID: PMC4669981.

5. Speer, R.M., Wise, C.F., Young, J.L., Aboueissa, A.M., Martin Bras, M., Barandiaran, M., Bermúdez, E., Márquez-D'Acunti, L., Wise, Sr., J.P. (2018). The cytotoxicity and genotoxicity of particulate and soluble hexavalent chromium in leatherback sea turtle lung cells. Aquatic Toxicology. 198:149157. PMID: 29547730. PMCID: PMC5915330.

6. Young, J.L., Cai, L., States, J.C. (2018). Impact of Prenatal Arsenic Exposure on Chronic Adult Diseases. Systems Biology in Reproductive Medicine. 64(6):469-483. PMID: 29873257. PMCID: PMC6291241 .

7. Liang, Y., Young, J.L., Kong, M., Tong, Y., Qian, Y., Freedman, J.H., Cai, L. (2019). Gender Differences in Cardiac Remodeling Induced by a High-Fat Diet and Lifelong, Low-Dose Cadmium Exposure. Chemical Research in Toxicology. 32(6):1070-108. PMID: 30912652. PMCID: PMC7060501.

8. Zhang, Y., Young, J.L., Cai, L., Tong, Y.G., Miao, L., Freedman, J.H. (2019). Chronic exposure to arsenic and high fat diet induces sex-dependent pathogenic effects on the kidney. Chemico-Biological Interactions. 310:108719 PMID: 31238026. PMCID: PMC7087439.

9. Young, J.L., Yan, X., Xu, J., Yin, X., Zhang, X., Arteel, G.E., Barnes, G.N., States, C.J., Watson, W.H., Kong, M., Cai, L., Freedman, J. (2019). Cadmium and High-Fat Diet Disrupt Renal, Cardiac, and Hepatic Essential Metals. Scientific Reports. 9(1):14675. PMID: 31604971. PMCID: PMC6789035.

10. Jiang, S., Young, J.L., Wang, K., Qian, Y., Cai, L. (2020). Diabetic-induced alterations in hepatic glucose and lipid metabolism: The role of type 1 and type 2 diabetes mellitus (Review). Molecular Medicine Reports. 22(2):603611. PMID: 32468027. PMCID: PMC7339764.

11. Young, J.L., Cai, L. (2020). Implications for Prenatal Cadmium Exposure and Adverse Health Outcomes in Adulthood. Toxicology and Applied Pharmacology. 403, 115161. PMID: 32721433 PMCID: PMC7453094.

\section{Book Chapters}

1. Wise, Jr., P., Wise, J.T., Wise, C.F., Young, J.L., Wise, Sr., J.P. Organizations. In: Information Resources in Toxicology. 5th ed. Wexler P, editor. Cambridge, MA: Elsevier; 2020. Chapter 65; p.643-671. 


\section{Abstracts}

1. Young, J.L., Holmes, A.L., Qin, Q., Xie, H., and Wise, Sr., J.P. Zinc Chromate Induces Spindle Assembly Checkpoint Bypass and Chromosome Instability in Human Lung Cells. Proceedings of the University of Southern Maine's Student Research Day: April, 2008.

2. Young, J.L., Holmes, A.L., Qin, Q., Xie, H., and Wise, Sr., J.P. Zinc Chromate Induces Spindle Assembly Checkpoint Bypass and Chromosome Instability in Human Lung Cells. Proceedings of the Toxics and Tomorrow's Children Conference at University of Southern Maine: March, 2008.

3. Young, J.L., Holmes, A.L., Qin, Q., Xie, H., and Wise, Sr., J.P. Zinc Chromate Induces Spindle Assembly Checkpoint Bypass and Chromosome Instability in Human Lung Cells. Proceedings of the American Association for Cancer Research 49: 4729, 2008.

4. Young, J.L., Wise, Jr. J.P., Wise, J., Jeevaragen, A., Wallace, W., Hammond, D., Shehata, T., and Wise, Sr. J.P. Lunar Dust and its Components are Cytotoxic to Human Lung Cells. Proceedings of the Annual Meeting of Northeast Chapter of the Society of Toxicology, October, 2008

5. Young, J.L., Holmes, A.L., Qin, Q., Xie, H. and Wise, Sr., J.P. Zinc Chromate Induces Spindle Assembly Checkpoint Bypass and Chromosome Instability in Human Lung Cells. Presented at Making the Connection III: Toxics and Tomorrow's Children Conference, 2008.

6. Young, J.L., Wise, Jr. J.P., Wise, J., Jeevaragen, A., Wallace, W., Hammond, D., Shehata, T., and Wise, Sr. J.P. Lunar Dust and its Components are Cytotoxic to Human Lung Cells. Toxicological Sciences 108:1778, 2009. March, 2009.

7. Young, J.L., Holmes, A.L., Qin, Q., Xie, H., and Wise, Sr., J.P. Zinc Chromate Induced Spindle Assembly Checkpoint Bypass and Chromosome Instability in Human Lung Cells. Proceedings of the $36^{\text {th }}$ Annual Biological and Medical Science Symposium, April, 2009.

8. Young, J.L., Wise, Jr., J.P., Wise, J., Jeevaragen, A., Wallace, W., Hammond, D., Shehata, T., and Wise, Sr., J.P. Lunar Dust and its Components are Cytotoxic to Human Lung Cells. Proceedings of the University of Southern Maine's Annual Thinking Matters, April, 2009.

9. Young, J.L., Wise, S.S., Xie, H., Wise, C.F., Fukuda, T., Guillette, Jr., L., Wise, Sr., J.P. A comparison of chromium cytotoxic and genotoxic in human, sea turtle, and alligator skin cells. Proceedings of the 5th Georgian Bay International Conference on Bioinorganic Chemistry, May 2015. 
10. Young, J.L., Wise, S.S., Xie, H., Wise, C.F., Fukuda, T., Guillette, Jr., L., Wise, Sr., J.P. A comparison of the cytotoxic and genotoxic effects of hexavalent chromium in human, aquatic reptile and aquatic mammal skin cells. Presented at the $46^{\text {th }}$ Annual Meeting of the Environmental Mutagenesis and Genomics Society, September 2015.

11.Young, J.L., Wise, S.S., Xie, H., Wise, C.F., Fukuda, T., Guillette, Jr., L., Wise, Sr., J.P. A comparison of the cytotoxic and genotoxic effects of hexavalent chromium in human, aquatic reptile and aquatic mammal skin cells. Presented at Research! Louisville, October 2015.

12. Young, J.L., Wise, S.S., Xie, H., Wise, C.F., Fukuda, T., Wise, Sr., J.P. Whale Cells May Have More Efficient Cellular Mechanisms against Chromium-Induced Genotoxicity than Both Human and Turtle Cells. Presented at the $21^{\text {st }}$ Biennial Meeting of the Society of Marine Mammalogy, December 2015.

13. Young, J.L., Wise, S.S., Wise, C.F., Fukuda, T., and Wise, Sr., J.P. Insights into Chromate Genotoxicity from a One Environmental Health Perspective: Alligators, Turtles, and Whales Oh My! Presented at the $55^{\text {th }}$ Annual Meeting of the Society of Toxicology, March 2016.

14. Young, J.L., Wise, S.S., Xie Wise, C.F., Fukuda, T., and Wise, Sr., J.P. A One Environmental Health Perspective: Insight into the Cytotoxic and Genotoxic Effects of Hexavalent Chromium in Human, Aquatic Reptile and Aquatic Mammal Skin Cells. Presented at the $47^{\text {th }}$ Annual Meeting of the International Association for Aquatic and Animal Medicine, May 2016.

15. Young, J.L., Poole, L.G., Nguyen, C.T., and Arteel, G.E. Exploring the Impact of Chromium Exposure on Macrophage Polarization: Implications for the Innate Immune Response. Presented at Research! Louisville, October 2016.

16. Young, J.L., Burke, T.G., Freedman, J., Watson, W.H., Cai, L., Merchant, M.L., States, C.J., Arteel, G.E. Effects of Early Life Chronic Exposure to Arsenic and Cadmium on the Development of Adult Cardiometabolic Syndrome. Presented at Research! Louisville, September 2017.

17. Young, J.L., Burke, T.G., Freedman, J., Watson, W.H., Cai, L., Merchant, M.L., States, C.J., Arteel, G.E. Effects of Early Life Chronic Exposure to Arsenic or Cadmium on the Development of Adult Metabolic Syndrome: Initial Characterization of Hepatic Changes. Presented at the Annual Meeting of the Ohio Valley Chapter of the Society of Toxicology, Perdue University, Lafayette, Indiana. December 2017.

18. Young, J.L., Burke, T.G., Freedman, J., Watson, W.H., Cai, L., Merchant, M.L., States, C.J., Arteel, G.E. Effects of Early Life Chronic Exposure to 
Arsenic or Cadmium on the Development of Adult Metabolic Syndrome: Initial Characterization of Hepatic Changes. Presented at the Graduate Student Regional Research Conference, Louisville Kentucky, March 2018.

19. Young, J.L., Burke, T.G., Freedman, J., Watson, W.H., Cai, L., Merchant, M.L., States, C.J., Arteel, G.E. Effects of Early Life Chronic Exposure to Arsenic and Cadmium on the Development of Adult Cardiometabolic Syndrome. Presented at the $56^{\text {th }}$ Annual meeting of the Society of Toxicology, San Antonio, Texas, March 2018.

20. Young, J.L., Freedman, J.H, Watson, W.H., Kong, K., Arteel, G.E., Cai, L. Effects of Whole Life Cadmium Exposure on the Development of NonAlcoholic Fatty Liver Disease in Male and Female Mice. Presented at Research! Louisville, October 2018.

21.D'Angelo, B., Tong, Y.G., Young, J.L., Freedman, J.H. The Effects of Cadmium on Kidney Tissue. Presented at Research! Louisville, October 2018.

22.Zhou, B., Gentry, A., Pagidas, K., Young, J.L., Kong, M., Cai, L., Freedman, J.H. A Long-Term Cadmium Exposure Impairs Spermatogenesis, Increasing Apoptosis in Mice Testis Independent of High Fat Diet. Presented at Research! Louisville, October 2018.

23.Zhang, Y., Young, J.L., Kong, M., Cai, L., Freedman, J.H. Chronic exposure to arsenic and high fat diet induces sex-dependent effects in mouse kidney. Presented at Research! Louisville, October 2018.

24. Yuan, F., Wu, T., Young, J.L., Fan, H., Li, Y., Lu, L., Liu, J., Cai, L., Chen, SY. Maternal Preconception Exposure to Cadmium Induces Apoptosis in Mouse Embryos by Decreasing DNA Methylation at the Promoter Regions of the Pro-apoptotic Genes. Presented at Research! Louisville, October 2018.

25. Watson, W.H., Young, J.L., Burke, T.J., Kalbfleisch, T., Cai, L., States, J.C., Arteel, G.E., Freedman, J.H. Sexually Dimorphic Hepatic Responses to Environmental Arsenic Exposure in a Mouse Model of Non-Alcoholic Fatty Liver Disease. Presented at Research! Louisville, October 2018.

26. Freedman, J.H., Young, J.L., Yan, X., Xu, J., Yin, X., Zhang, X., Arteel, G.E., Barnes, G., States, J.C., Watson, W.H., Kong, M., Cai, L. Cadmium and HighFat Diet Disrupt Renal, Cardiac and Hepatic Essential Metals. Presented at Research! Louisville, October 2018.

27. Young, J.L., Yan, X., Xu, J., Yin, X., Zhang, X., Arteel, G.E., Barnes, G.N., States, J.C., Watson, W., Kong, M., Freedman, J.H., Cai, L. Cadmium and High-Fat Diet Disrupt Renal, Cardiac and Hepatic Essential Metal 
Homeostasis. Presented at the $10^{\text {th }}$ Conference on Metal Toxicity \& Carcinogenesis, October 2018.

28. Young, J.L., Liang, Y., Merchant, M.L., Arteel, G.E., Freedman, J.H., and Cai, Lu. Sex-Dependent Effects of Early Life Cadmium Exposure and High Fat Diet on the Liver, Heart and Kidney. Presented at the $58^{\text {th }}$ Annual Meeting of the Society of Toxicology, March 2019.

29. Watson, W.H., Young, J.L., Burke, T.J., Kalbfleisch, T., Cai, L., States, J.C., Arteel, G.E., Freedman, J.H. Sexually Dimorphic Hepatic Responses to Environmental Arsenic Exposure in a Mouse Model of Non-alcoholic Fatty Liver Disease. Presented at the $58^{\text {th }}$ Annual Meeting of the Society of Toxicology, March 2019.

30.Zhang, Y., Young, J.L., Kong, M., Freedman, J.H., and Cai, L. Chronic Exposure to Arsenic and High-Fat Diet Induces Sex-Dependent Renal Effects. Presented at the $58^{\text {th }}$ Annual Meeting of the Society of Toxicology, March 2019.

31. Wise, J.P., Croom-Perez, T.J., Wise, C.F., Young, J.L., Wise, S.S., UrbanR, J., Lopez, C., Markley, L.C., Bras, M.M., Speer, R.M., Isousi, I.M., Aboueissa, A., Wise, J.T.F. A Whale of a Tale: A One Environmental Health Approach to Study Metal Pollution in the Sea of Cortez. Presented at the $58^{\text {th }}$ Annual Meeting of the Society of Toxicology, March 2019.

32. Young, J.L., Zhou, B., Zhou, L., Cai, L. The Effects of Whole Life, Low Dose Cadmium Exposure on High Fat Diet-Induced Metabolic Disease and Its Modulation by Zinc. Presented at The XV International Congress of Toxicology (ICTXV) in Honolulu, Hawaii July 2019.

33. Cai, L., Liang, Y., Young, J.L., Kong, M., Tong, Y., Qian, Y., and Freedman, J.H. Gender Differences in Cardiac Remodeling Induced by a High-Fat Diet and Whole-Life, Low-Dose Cadmium Exposure. Presented at The XV International Congress of Toxicology (ICTXV) in Honolulu, Hawaii July 2019.

34. Young, J.L., Zhou, B., Zhou, L., Cai, L. The Effects of Whole Life, Low Dose Cadmium Exposure on High Fat Diet-Induced Metabolic Disease and Its Modulation by Zinc. Presented at Research!Louisville, September 2019.

35. Young, J.L., Zhou, B., Zhou, L., Zhou, W., Men, H., and Cai, L. The Role of Dietary Zinc in Cadmium-Enhanced Non-Alcoholic Fatty Liver Disease. Presented at The International Society for Trace Element Research in Humans XIII conference in Bali, Indonesia September 2019 (Oral presentation). 
36. Young, J.L., Zhou, B., Zhou, L., Zhou, W., Men, H., and Cai, L. The Role of Dietary Zinc in Cadmium-Enhanced Non-Alcoholic Fatty Liver Disease. Presented at the Annual Meeting of the Ohio Valley Chapter of the Society of Toxicology at Procter \& Gamble Mason Business Center (MBC) in Mason, Ohio October 2019 (Oral presentation).

37. Young, J.L., Zhou, B., Zhou, L., Zhou, W., Men, H., and Cai, L. The Effects of Whole Life, Low Dose Cadmium Exposure on High Fat Diet-Induced Metabolic Disease and Its Modulation by Zinc. Presented at the Annual NIEHS Superfund Research Program Meeting in Seattle, Washington November 2019.

38. Young, J.L., Wise, Jr., J.P., Wise, J.T.F., Wise, C.F., Wise, S.S., Zhu,C., Browning, C.L., Zheng, T., Perkins, C.R., Gianios Jr., C., Xie, H., Cai, L., and Wise, Sr., J.P. A Whale of a Tale: A 3-Year Study of Metals in Gulf of Maine Whales. Presented at the World Marine Mammal Conference in Barcelona, Spain December 2019.

39. Croom-Perez, T.J., Young. J.L., Xu, J., Meaza, I., Lu, H., Wise, S.S., Cai, L., and Wise, Sr., J.P. Characterizing a mouse model for the effects of whole life, low dose cadmium exposure and high fat diet on the lung. Presented at the 59th Annual Society of Toxicology (SOT) Meeting Anaheim, California March $\underline{2020 .}$

40. Young, J.L., and Cai, L. The Role of Dietary Zinc in Cadmium-Enhanced Non-Alcoholic Fatty Liver Disease. Presented at the 59th Annual Society of Toxicology (SOT) Meeting Anaheim, California March 2020.

41. Young, J.L., and Cai, L. Zinc supplementation rescues cadmiumexacerbated, high fat diet-induced NAFLD. Presented at the Annual Meeting of the Ohio Valley Chapter of the Society of Toxicology, hosted by the University of Louisville, November 2020.

42. Young, J.L., and Cai, L. Sexual Dimorphism of Cadmium-Exacerbated, DietInduced Liver Disease. Presented at the Annual NIEHS Superfund Research Program Meeting, hosted by the University of Arizona, December 2020. 